\title{
Development of aptamer technologies for the detection of dopamine and other biological molecules
}

\author{
By
}

\section{Ryan Walsh}

A thesis submitted to

The Faculty of Graduate Studies and Research

in partial fulfilment of

the degree requirements of

Doctor of Philosophy

in

Chemistry

\author{
Department of Chemistry \\ Carleton University \\ Ottawa, Ontario, Canada
}

C 2012 - Ryan Walsh 
Library and Archives

Canada

Published Heritage

Branch

395 Wellington Street

Ottawa ON K1A ON4

Canada
Bibliothèque et

Archives Canada

Direction du

Patrimoine de l'édition

395 , rue Wellington

Ottawa ON K1A ON4

Canada
Your file Votre référence

ISBN: $978-0-494-93700-6$

Our file Notre référence

ISBN: 978-0-494-93700-6
NOTICE:

The author has granted a nonexclusive license allowing Library and Archives Canada to reproduce, publish, archive, preserve, conserve, communicate to the public by telecommunication or on the Internet, loan, distrbute and sell theses worldwide, for commercial or noncommercial purposes, in microform, paper, electronic and/or any other formats.

The author retains copyright ownership and moral rights in this thesis. Neither the thesis nor substantial extracts from it may be printed or otherwise reproduced without the author's permission.
AVIS:

L'auteur a accordé une licence non exclusive permettant à la Bibliothèque et Archives Canada de reproduire, publier, archiver, sauvegarder, conserver, transmettre au public par télécommunication ou par l'Internet, prêter, distribuer et vendre des thèses partout dans le monde, à des fins commerciales ou autres, sur support microforme, papier, électronique et/ou autres formats.

L'auteur conserve la propriété du droit d'auteur et des droits moraux qui protege cette thèse. $\mathrm{Ni}$ la thèse ni des extraits substantiels de celle-ci ne doivent être imprimés ou autrement reproduits sans son autorisation.
In compliance with the Canadian Privacy Act some supporting forms may have been removed from this thesis.

While these forms may be included in the document page count, their removal does not represent any loss of content from the thesis.
Conformément à la loi canadienne sur la protection de la vie privée, quelques formulaires secondaires ont été enlevés de cette thèse.

Bien que ces formulaires aient inclus dans la pagination, il n'y aura aucun contenu manquant. 


\section{Abstract}

The experiments detailed in this thesis describe the application of DNA aptamerbased technologies for the detection of the small molecules, dopamine and microcystin $L R$, as well as for the modulation of the enzymatic activity of kallikrein.

Using dopamine as a major focus of this research, we were able to demonstrate that a simple RNA aptamer structure, such as the RNA dopamine aptamer, can be mimicked by a DNA homolog. We were also able to show through SELEX that while the DNA homolog of the RNA aptamer was observed to have a fairly good binding affinity, the sequence space of potential DNA aptamers for dopamine is still quite vast, as none of the isolated DNA sequences bore any resemblance to the RNA dopamine aptamer.

We also investigated the utilization of DNA aptamers in electrochemical biosensors by functionalizing glassy carbon electrodes with the DNA homolog of the RNA dopamine aptamer. This demonstrated the potential to generate highly selective electrochemical biosensors by coupling the electrochemical signal to selective aptamer binding interactions.

Additional studies detailed the SELEX based production. of DNA aptamers for microcystin LR, a hepatotoxin produced by algae blooms and of particular interest in surveillance of drinking water quality.

The final study looks at the ability of DNA aptamers to modulate the enzymatic activity of human plasma kallikrein, an enzyme involved in the angiotensin pathway. 
While most therapeutics that modulate the activity of enzymes are inhibitory as they provide simple blockage of the enzymatic active site, the select.on method described in this study was able to demonstrate that the certain DNA pools produced were able to activate while others inhibited kallikrein. Production of aptamers which can activate or inhibit the activity of enzymes presents a new avenue for producing tools for probing metabolic processes and may lead to useful new therapeutics.

These studies further demonstrate the utility of DNA as a functional nanomaterial that has the potential to revolutionize bionanotechnologies. 


\section{Acknowledgements}

I would like to thank my supervisor Dr Maria DeRosa for providing me with the guidance I needed if didn't always want and allowing me to come into contact with the wonderful group of people that have made the last five years both fun and exciting.

I would like to thank the guys back in Nova Scotia. Thanks Sultan and Earl your support has shaped me as a researcher and given me the confidence to inflict my often misguided opinions on others. While I thank you for that I'm not sure the rest of the world will.

I would like to thank the people who worked with me on the various research projects I have been involved in during my time at Carleton and despite my help some seem to have actually worked .... thanks for keeping things on track:

Amirah Yaakob, Dave Blair, Erin McConnell, Hechen '.ui, Josh Chan, Sameer Dedhar, Tariq Francis, Tasjeel Ansari, Uyen Ho, Xai Li Wang, Yasir Sultan.

Thanks particularly to Yasir for his help with new techniques and for making the evenings entertaining.

I would also like to thank all the rest of the past and present members of the DeRosa Lab which have made the last few years a great experience:

Anwar Howemid, Amanda Foster, Amanda Giamberardino, Charlotte Bradley, David Coffey, Elyse Bernard, Erika Revesz, Jen Lee, Johny Abboud, Maureen McKeague, 
Mehwish Khalid, Mike Beking, Peter Pallister, Shirley Chang, Stephen Boulton, Sara Hosseini, Siddhartha Nandi, Yasmine Miguel, Xueru Zhang.

In Particular thanks to the other gradstudents, John Palko, Monica Gill, Jason Coyle and anyone I've forgotten, who shared the highs and lows that comes with being in grad school and made them fun and enjoyable.

Finally I would like to thank my family for all the patience they have had during my studies, I couldn't have done it without you thank you for your love and support.

Thank you everyone 


\section{Contents}

Chapter 1: DNA as a programmable nanomaterial.............................................................. 1

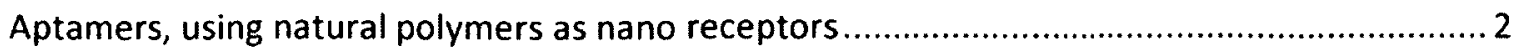

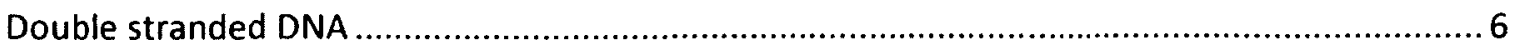

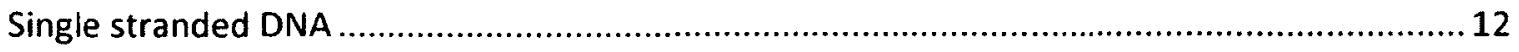

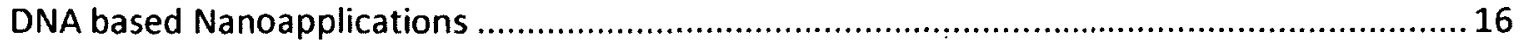

DNA as a multifunctional nanomaterial in biological applications ...................................... 17

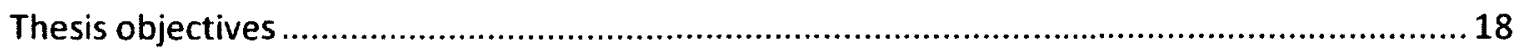

Chapter 2: Retention of function in the DNA homolog of the RNA dopamine aptamer............20

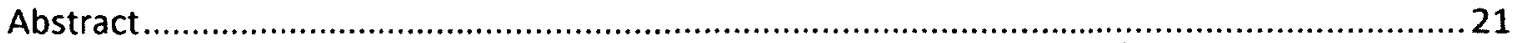

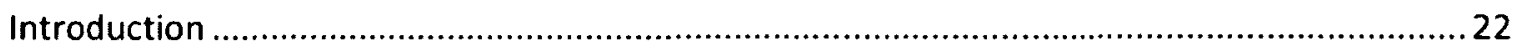

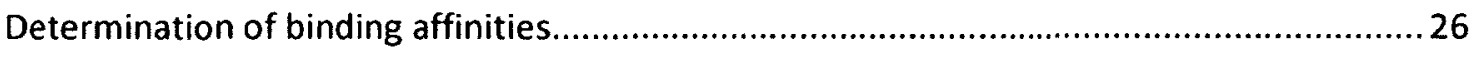

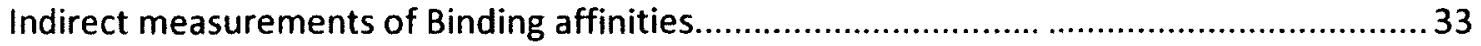

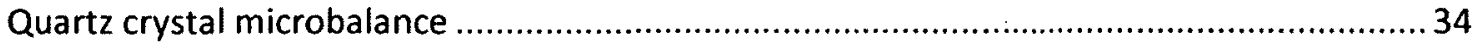

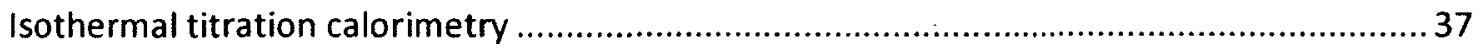

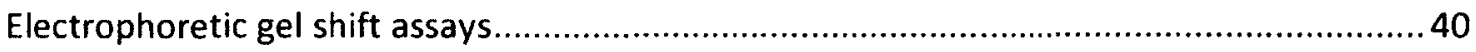

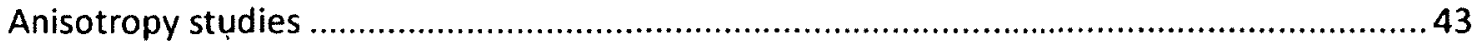

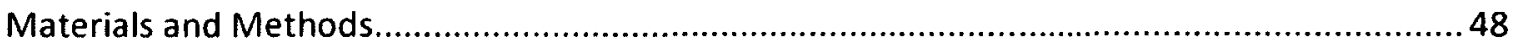

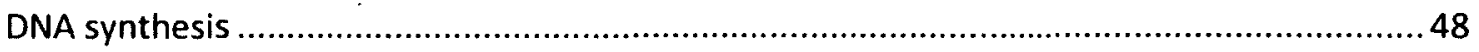

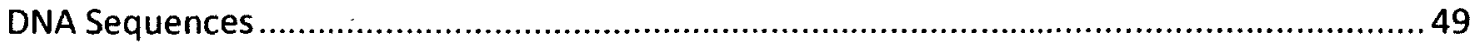

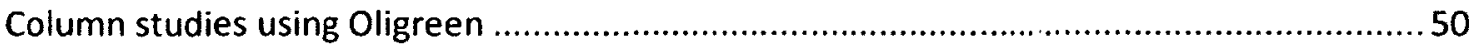

Column studies using fluorescein labelled aptamer........................................................ 51

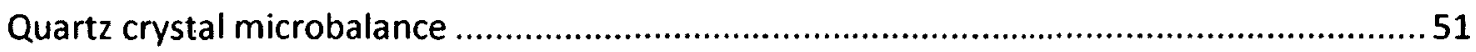

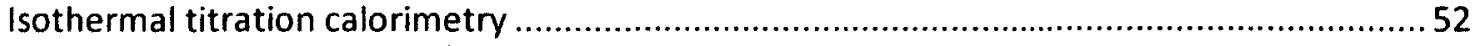

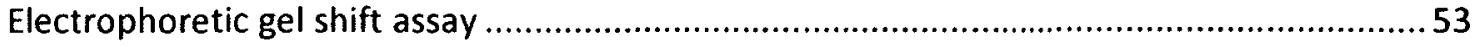

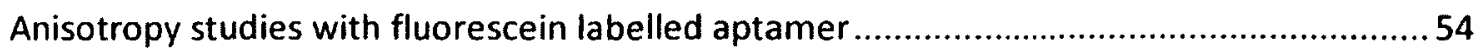

Anisotropy studies using target as the reporter molecule .............................................55

Data fitting for dissociation constant determination ....................................................55

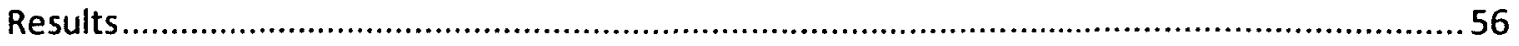

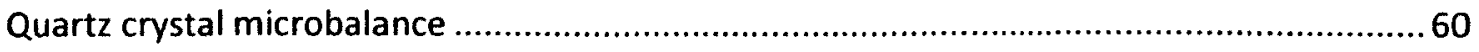

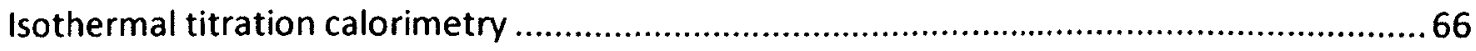


Gel shift assays

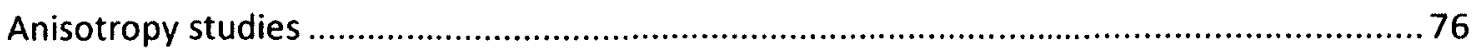

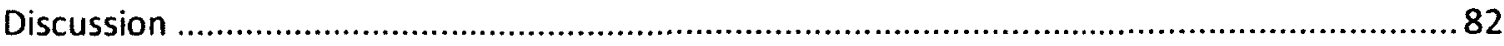

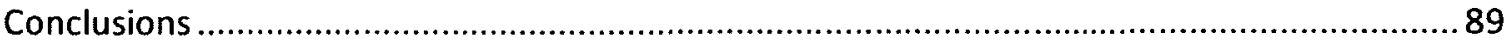

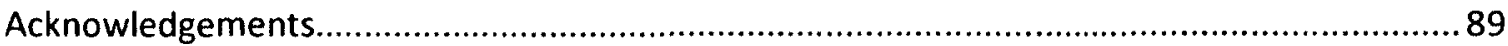

Chapter 3: Dopamine detection using aptamer functionalized glassy carbon electrodes ...........91

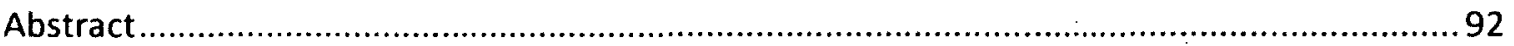

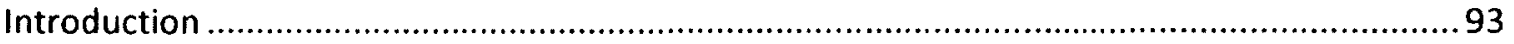

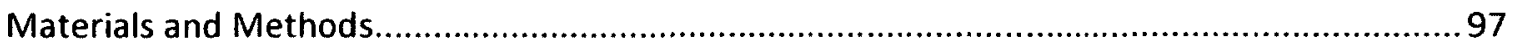

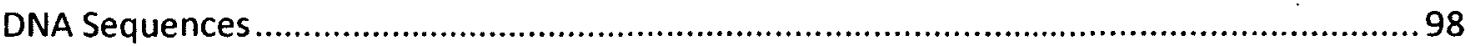

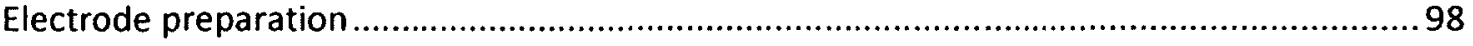

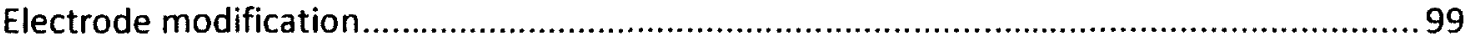

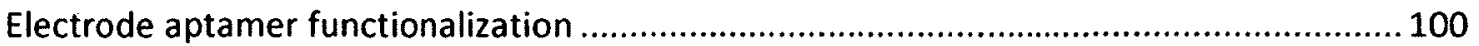

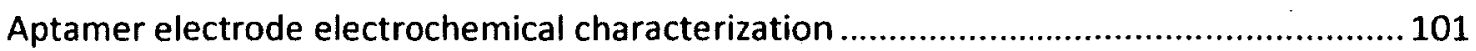

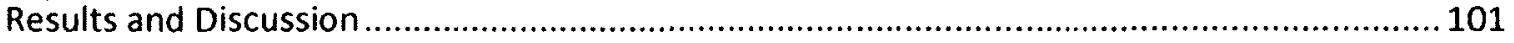

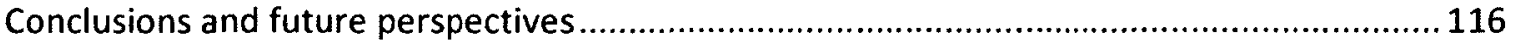

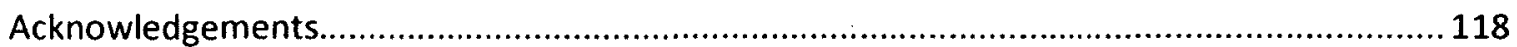

Chapter 4 Systematic Evolution of Ligands by EXponential enrichment (SELEX) for DNA aptamers

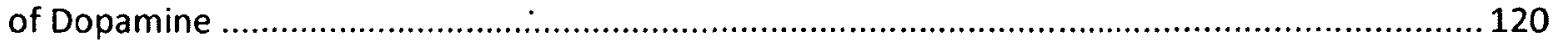

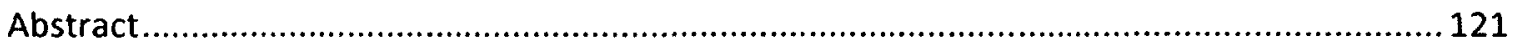

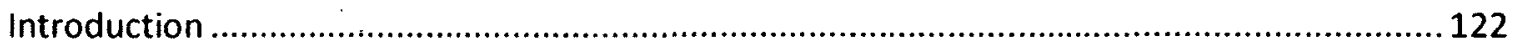

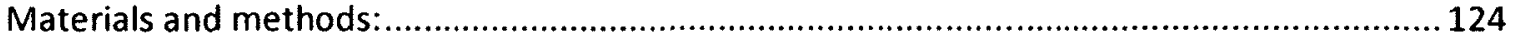

DNA based selection for dopamine aptamers ................................................................ 126

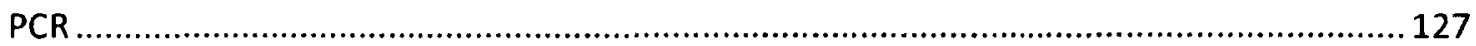

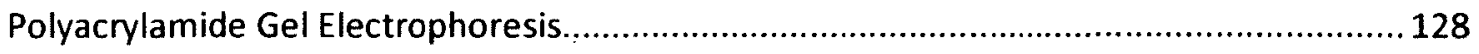

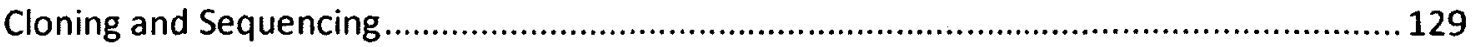

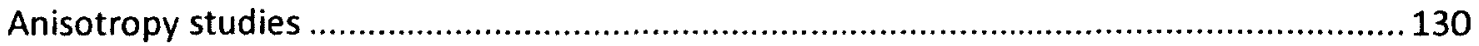

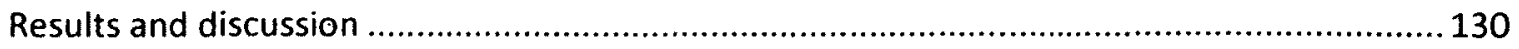

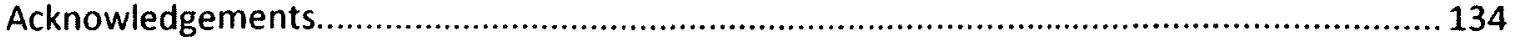

Chapter 5: Systematic Evolution of Ligands by EXponential enrichment (SELEX) for DNA

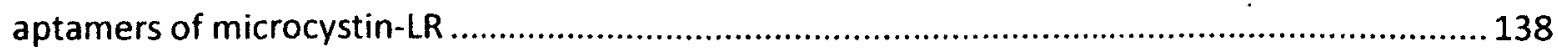

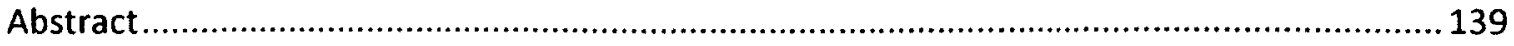


Introduction

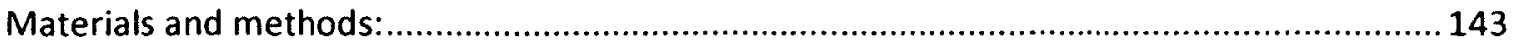

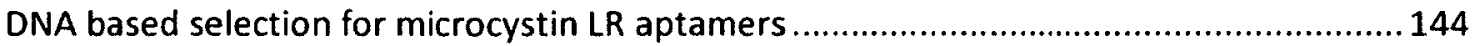

PCR

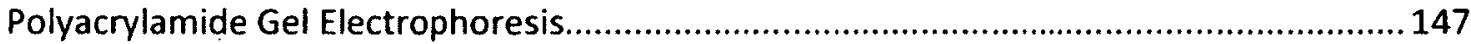

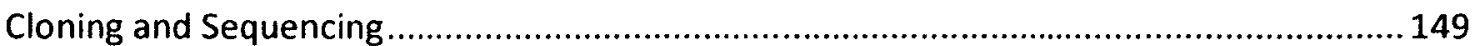

Anisotropy studies with fluorescein labelled aptamer ..................................................... 150

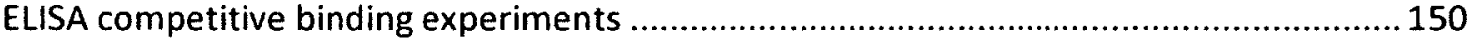

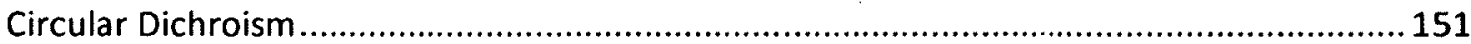

Data fitting for dissociation constant determination ............................................... 152

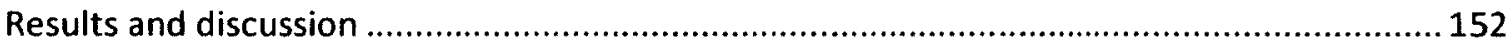

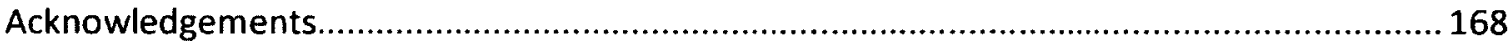

Chapter 6: Systematic Evolution of Ligands by EXponential enrichment (SELEX) for DNA aptamers which modulate the catalytic activity of Kallikrein ............................................... 170

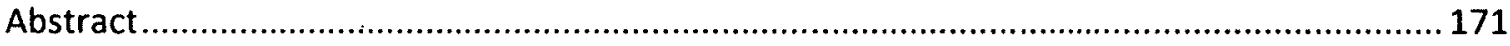

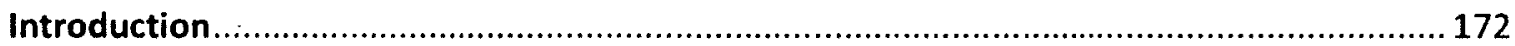

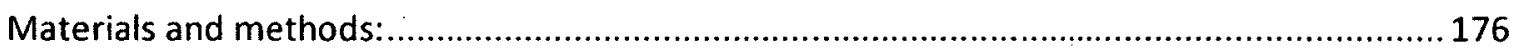

Selection for Kallikrein activity modifying aptamers .................................................... 177

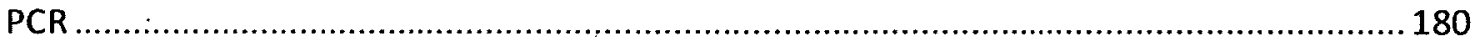

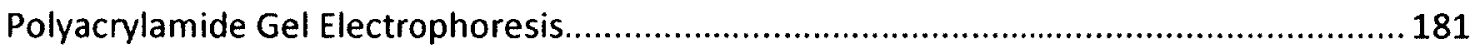

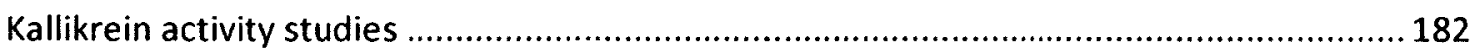

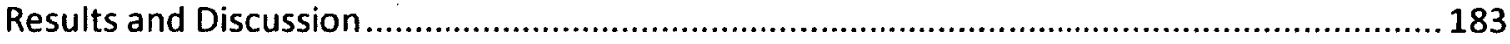

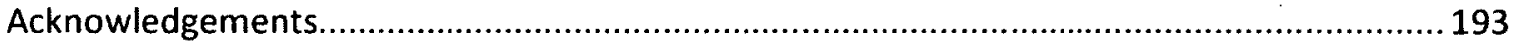

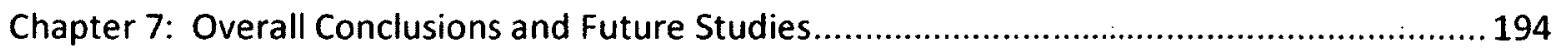

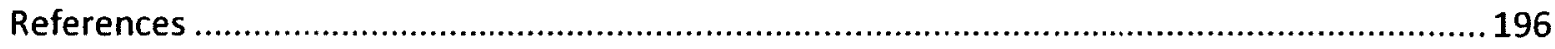

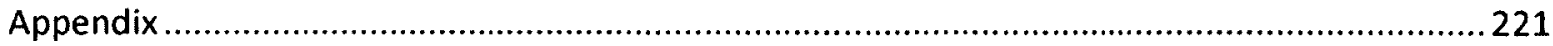




\section{Table of Figures}

\section{Chapter 1}

Figure 1.1. Schematic representation of the SELEX process. Initially the pool of random sequences is exposed to a target of interest, followed by washing away of sequences with no low affinity for the target. The sequences of interest are then separated from the target and amplified to produce a new pool. This new pool is then reintroduces to the target to sort higher affinity sequences from lower affinity sequences. This cycle is repeated multiple times to enrich the number of high affinity sequences present before a sample of the pool is sequenced to determine the primary aptamer sequences which can be characterized by their binding affinity for the target.

Figure 1.2. Simple scaffold structure generated with CADNANO a program for templating DNA origami structures (Douglas et al., 2009, http://cadnano.org/).

Figure 1.3. Known variations of the DNA double helix A) A DNA is very similar to the coiling produced by RNA, B) B DNA is the most common helical structure with a positive twist of $36^{\circ}$ per base pair (Lu and Olson, 2008). C) The more unusual Z DNA has been found in regions of high GC content and has a negative twist of $-60^{\circ}$ per base pair (Lu and Olson, 2008). This figure was generated with Web 3DNA (Zheng et al., 2009; http://w3dna.rutgers.edu) and Jmol (Herráez, 2006; Imol: an open-source Java viewer for chemical structures in 30. http://www.jmol.org//. Models were produced using the DNA sequence atcgatcgatcgatcg for A and B DNA while eight $C G$ repeats were used to generate the $Z$ DNA construct.

Figure 1.4. Structure of deoxyribonucleic acid (DNA) depicting the two purines, adenine (A) and guanine $(G)$, base pairing to their respective partner pyrimidines, thymine $(T)$ and Cytosine (C). This Watson-Crick binding forms the basis for DNA sequence complement binding which produces the double stranded helix. Figure generated with Web 3DNA (Zheng et al., 2009; http://w3dna.rutgers.edu) and Jmol (Herráez, 2006; Jmol: an open-source Java viewer for chemical structures in 30. http://www.jmol.org/)

Figure 1. 5. Schematic representation of some of the tertiary structures produced by single stranded DNA. The hairpin (A) is produced by looping back of a single strand while more complex structures such as pseudo knots (B) can occur when base pairing involves multiple looping events. These structures are not constrained by Watson-Crick base pairing associated with the double helix and may result in even more complex base pairing such as is observed with g-quadraplexes for their stability (C-D) (Parkinson, 2006).

Figure 1. 6. Schematic of the edges used to designate the interactions between nucleobases. Each base has a Watson-Crick edge and a sugar edge. The difference in structure between the purine bases and pyrimidine bases is distinguished with the designation of the Hoogsteen edge and the C-H edge (Leontis et al., 2002). 


\section{Chapter 2}

Figure 2.1. The secondary structure of the RNA dopamine aptamer proposed by Mannironi, et al., (1997) displayed with a tertiary structure of the aptamer generated using MC-Fold \& MCSym (Parisien and Major, 2008) and rendered using pymol (DeLano, 2002). Yellow highlighted regions correspond to the dopamine binding domain. Complementary and invariant bases in the loops that are required for dopamine binding are indicated in red and blue, respectively...24 Figure 2.2. Schematic of the tertiary structure of the B12 RNA aptamer along with the crystal structure reported by Sussman, et al., (2000). The structure demonstrates the complex fold providing a complex surface which interacts with vitamin B12. The figure was generated using the modeling program Jmol (Herráez, 2006; Jmol: an open-source Java viewer for chemical structures in 3D. http://www.jmol.org// and the crystal structure of the B12 aptamer complexed with B12, 1DDY (Sussman et al., 2000), in the protein Data bank (Berman et al., 2000). .25 Figure 2.3. Typical binding isotherm for receptor ligand interactions. The observed response is a relative scale produced by the method of detection and the sensitivity of the experimental method. A) As the concentration of ligand is increased the receptor binding spots are saturated the signal reaches a distinct maximum. B) In the presence if a nonspecific interaction, specific signal can be masked by the combind signal but the combine signal will not conform to the rectangular hyperbola expected of a simple binding isotherm

Figure 2.4. Schematic representation of the potential interaction of dopamine and the DNA homolog dopamine aptamer deposited on the gold electrode of the quartz crystal microbalance crystal. Change in frequency directly relate to the binding of the ligand to the receptor which increases resistance to the vibration of the resonator.

Figure 2.5. Schematic representation of the heat evolved from a binding reaction and the experimental setup of a isothermal titration apparatus where a sample and reference cell are kept at constant temperature by feedback heater systems that measure the change in heat by the energy required to re-establish equilibrium.

Figure 2.6. Schematic of the instrumentation involved in fluorescence anisotropy studies. Typically light is filtered to produce vertically oriented light for photoselective fluorophore excitation. Both the vertical $\left(l_{11}\right)$ and horizontal $\left(l_{\perp}\right)$ fluorophore emission intensities are collected at $90^{\circ}$ to the incident of the excitatory light source.

Figure 2.7. Schematic representation of the drop in signal emission intensity ( $m+$ ) resulting from the reduction of fluorophore $(\bar{\varnothing})$ rotational freedom. This reduction of rotational freedom is produced by the change in mass associated with the formation of a receptor ligand complex. The measurement of emission intensity in the parallel $\left(I_{\| 1}\right)$ and perpendicular $\left(I_{\perp}\right)$ orientations allows the collection of signal at increasing rotational distance from the excitation orientation. Introduction of molecules that binds to the fluorophore decreases the fluorophores rotational speed causing a reduction in the number of fluorescent molecules that will be able to reach the parallel $\left(I_{112}\right)$ and perpendicular $\left(I_{\perp 2}\right)$ orientations before emitting.

Figure 2.8. Analysis of the binding of the DNA homolog of the RNA aptamer to the dopamine column using Oligreen. Oligreen was used to quantify the amount DNA retained on the 
dopamine column as increasing concentrations of the aptamer were exposed to the dopamine column. The gray line represents the fit of the data to the binding isotherm and the insert represents the linearity between the observed and calculated values.

Figure 2.9. Analysis of the binding of the fluorescein labelled DNA homolog of the RNA aptamer to the dopamine column. The fluorescein tag was used to quantify the amount DNA retained on the dopamine column as increasing concentrations of the aptamer were exposed to the dopamine column. The dots represent the collected data while the gray line represents the fit to Equation 17 accounting for the specific and nonspecific binding. The black line represents the specific aptamer binding and the inset represents the linearity between the observed and calculated values. 59

Figure 2.10. Chemisorption of the thiolated aptamer on the gold electrode pad of the QCM crystal. Decrease in frequency at which the QCM crystal vibrated was used to monitor the deposition of the thiolated aptamer by introducing two successive incubations of a $0.4 \mathrm{mM}$ aptamer solution in buffer.

Figure 2.11. Quartz crystal microbalance study of the potential binding between the aptamer and dopamine. The frequency of the aptamer functionalized crystal was monitored as increasing concentrations of dopamine were introduced to the flow cell $(0,0.05,0.16,0.49,1.6$, 4.4 and $13 \mu \mathrm{M})$. The insert depicts a plot of the equilibrium frequency plotted against dopamine concentration.

Figure 2.12. Quartz crystal microbalance study of the potential binding between the DNA B12 aptamer and increasing concentrations of B12. The initial three equilibrations corresponded to buffer, followed by the introduction of $0.02,0.2,2,20,200$ and $2000 \mu \mathrm{M}$ B12. The inset plot represents resting potential versus concentration of $\mathrm{B} 12$.

Figure 2.13. Isothermal titration microcalorimetric (a) raw data for the analysis of potential dopamine and aptamer interactions using ten $12 \mu \mathrm{l}$ injections of $0.47 \mathrm{mM}$ dopamine into 1.4 $\mathrm{mLs}$ of $20 \mu \mathrm{M}$ dopamine aptamer and (b) the associated integrated heat plot.

Figure 2.14. Isothermal titration microcalorimetric analysis of the (a) raw data profile for dopamine diffusion into buffer using ten $12 \mu$ injections of $0.47 \mathrm{mM}$ dopamine and (b) the associated integrated heat plot. .68

Figure 2.15. Isothermal titration microcalorimetric (a) raw data for the analysis of potential dopamine and aptamer interactions using thirty $6 \mu \mathrm{l}$ injections of $0.47 \mathrm{mM}$ dopamine into 1.4 $\mathrm{mLs}$ of $20 \mu \mathrm{M}$ dopamine aptamer and (b) the associated integrated heat plot. 70 Figure 2.16. Isothermal titration microcalorimetric with ascorbic acid omitted from the buffer (a) raw data for the analysis of potential dopamine and aptamer interactions using thirty $6 \mu l$ injections of $0.47 \mathrm{mM}$ dopamine into $1.4 \mathrm{mLs}$ of $20 \mu \mathrm{M}$ dopamine aptamer and (b) the associated integrated heat plot. .71 Figure 2.17. Isothermal titration microcalorimetric analysis of the (a) raw data profile for buffer addition to the aptamer using thirty $6 \mu \mathrm{l}$ injections of buffer into $1.4 \mathrm{mLs}$ of $20 \mu \mathrm{M}$ dopamine aptamer and (b) the associated integrated heat plot.

Figure 2.18. Fluorescein labelled DNA homologue of the RNA Dopamine aptamer $(4 \mu \mathrm{M})$ run on a $4 \%$ acrylamide gel at a constant 45 mAmps for 1.5 hours. Lane 1 and 2 contain just the 
aptamer while lane 3 and 4 were run in the presence of $100 \mathrm{mM}$ dopamine to ensure the presence of the dopamine bound conformation. The relative fluorescence of the fluorescein labelled aptamer was examined with an Alphalmager EC System equip with a $537 / 35 \mathrm{~nm}$ filter.

Figure 2.19. Fluorescein labelled DNA homologue of the RNA Dopamine aptamer $(4 \mu \mathrm{M})$ run on a $4 \%$ acrylamide gel at a constant 45 mAmps for 1.5 hours. Lane 1-9 contained dopamine of decreasing concentration: $13,1.3 \mathrm{mM}, 130,39,13,3.9,1.3,0.1 \mu \mathrm{M}$ and no dopamine. The relative fluorescence of the fluorescein labelled aptamer was examined with an Alphalmager EC System equip with a $537 / 35 \mathrm{~nm}$ filter. .75

Figure 2.20. Fluorescent anisotropy of the fluorescein labelled DNA dopamine aptamer (10 $\mu \mathrm{M})$ exposed to increasing concentrations of dopamine $(0.14,0.73,3.6,18,91,456 \mu \mathrm{M}, 2.28,5.96$ and $59.6 \mathrm{mM}$ ).

Figure 2.21. Fluorescent anisotropy of the fluorescein labelled B12 aptamer DNA homolog (10 $\mu \mathrm{M})$ exposed to increasing concentrations of $B 12(0.1,1,10,100$ and $1000 \mu M)$. The inset shows the agreement between the observed and the calculated anisotropy values. .78

Figure 2.22. Fluorescent anisotropy binding curve for the DNA form of the RNA dopamine aptamer and dopamine. Anisotropy values were collected using $0.1 \mu \mathrm{M}$ dopamine which was exposed to increasing concentrations of the aptamer $(0.27,2.7,27,270,2700,27000 \mathrm{nM})$. The inset shows the agreement between the observed and the calculated anisotropy values. .79 Figure 2.23. Assessment of the specificity of the DNA homolog. Comparison of the binding curves of DNA1 with dopamine (center) and two similar catechols, norepinephrine (left) and tyramine (right) indicated that the DNA aptamer has similar affinity for norepinephrine and dopamine but a much lower affinity for tyramine. 80

\section{Chapter3}

Figure 3.1. Reaction scheme for production of the dopamine aptamer functionalized glassy carbon electrode.

Figure 3.2. Glassy carbon electrode surface passivation through successive scans using 0.1M 4(trifloromethyl)benzenediazonium in acetonitrile and $0.1 \mathrm{M}$ 4-nitrobenzenediazonium in acetonitrile. This produced a heterogeneous trifluoromethylphenyl-nitrophenyl monolayer.. 104 Figure 3.3. Nitro to amine conversion of surface nitro groups was accomplished using repetitive scans between -0.4 and $-1.5 \mathrm{~V}$ in $0.1 \mathrm{M} \mathrm{KCl} \mathrm{10:90} \mathrm{ethanol} \mathrm{and} \mathrm{water.}$ 105 Figure 3.4. Cyclic voltammogram characterization $(0$ to $0.8 \mathrm{~V})$ of the passivation of a glassy carbon electrode. The response of the polished glassy carbon electrode to the catecholamine norepinephrine was compared to the signal produced after the surface passivation with the heterogeneous trifluoromethylphenyl-aminophenyl monolayer coating. 106 Figure 3.5. Square wave characterization $(0$ to $0.8 \mathrm{~V})$ of the passivation of a glassy carbon electrode. The response of the polished glassy carbon electrode was compared to the signal 
produced after the passivation of the surface with the heterogeneous trifluoromethylphenylaminophenyl monolayer coating. The observed signal after passivation was comparable to that of buffer alone. 107

Figure 3.6. Square wave voltomic characterization $(0-0.8 \mathrm{~V})$ of the relative signals produced by the dopamine aptamer functionalized electrode with $1 \mathrm{mM}$ dopamine and A) $1 \mathrm{mM}$ norepinephrine, B) $1 \mathrm{mM} \mathrm{L-dopa,} \mathrm{C)} 1 \mathrm{mM}$ 3,4-Dihydroxyphenylacetic acid, D) $1 \mathrm{mM}$ tyramine, E) $1 \mathrm{mM}$ serotonin. 109

Figure 3.7. Assessment of the electrode detection range for dopamine using A) square wave scans from $0-0.8 \mathrm{~V}$ in increasing concentrations of Dopamine $(50,100,500 \& 1000 \mu \mathrm{M})$. B) Standard curve plot of peak current at $0.18 \mathrm{~V}$ versus Dopamine concentration.

Figure 3.8. Square wave voltomic characterization $(0-0.8 \mathrm{~V})$ of the relative signals produced by the dopamine aptamer functionalized electrode with $1 \mathrm{mM}$ doparnine and a random DNA sequence functionalized electrode with $1 \mathrm{mM}$ dopamine.

Figure 3.9. Square wave voltomic characterization of the relative signal affect of mixing $1 \mathrm{mM}$ dopamine with A) $1 \mathrm{mM}$ ascorbic acid, B) $1 \mathrm{mM}$ Norepinepherine and C) $1 \mathrm{mM}$ Serotonin. 114

\section{Chapter 4}

Figure 4.1. Pool sequences were eluted from the dopamine selection column competitively using $0.1 \mathrm{M}$ dopamine. The bars represent the approximate percent of the original pool which was eluted from the dopamine column during the second third and fourth selection rounds. . 132

\section{Chapter 5}

Figure 5.1. Microcystins are cyclic heptapeptides which are produced by cyanobacteria. Over 80 variants of these molecules have been reported but are distinguished mainly by their amino acid substituent's in position $X$ and $Z$ of the ring (Campos and Vasconcelos, 2010). Microcystin LR (A) which is the most common form of these microtoxins has an arginine in position $Z$ and a leucine at position $X$. Microcystin LA (B) is distinguishable from microcystin LR by the presence of an alanine in position $Z$. 141

Figure 5.2. Elution profile from the $5^{\text {th }}$ selection round where the pool was initially washed from the microcystin LR column using selection buffer (W1-W3), followed by elution with hot water $\sim 90^{\circ} \mathrm{C}(\mathrm{E1}, \mathrm{E2})$ then elution with hot $3.5 \mathrm{M}$ Urea $\sim 90^{\circ} \mathrm{C}(E 3, \mathrm{E} 4)$. Bars represent the fluorescence of the collected fractions (excitation 490 emission $518 \mathrm{~nm}$ ). 154

Figure 5.3. Elution profile from the $6^{\text {th }}$ selection round where the pool was initially incubated with 100 fold excess microcystin LA before being exposed to the microcystin LR column. Fluoresces associated with the wash through (LA) followed by washes with the selection buffer 
(W1-W4) and then elution with hot $3.5 \mathrm{M}$ Urea $\sim 90^{\circ} \mathrm{C}$ (E1-ES). Bars represent the fluorescence of the collected fractions (excitation 490 emission $518 \mathrm{~nm}$ ). 155

Figure 5.4. Potential secondary structures, primary sequence not shown, for the three SELEX generated DNA sequences used in assessing the pools binding specificity towards microcystin LR. Structures were generated using the DNA settings of the RNAfold suite of the Vienna RNA Websuite (Gruber et al., 2008). 156

Figure 5.5. Fluorescent anisotropy binding curve for the fluorescently tagged $\mathrm{H} 12$ sequence and microcystin LR. Anisotropy values were collected using $0.57 \mathrm{nM} \mathrm{H} 12$ which was exposed to increasing concentrations of microcystin $\operatorname{LR}(0.05,0.5,5,50,500,5000 \mathrm{nM})$. The inset shows the agreement between the observed and the calculated anisotropy values. 158 Figure 5.6. CD study of the affinity of $\mathrm{H} 12$ for microcystin LR (A) repetitive scans of $\mathrm{H} 12$ with the concentrations of microcystin $L R$ varying $0.4 \mathrm{nM}$ to $1.6 \mu \mathrm{M}$. The insert represents the polynomial smoothing used to analyzing the peak around $220 \mathrm{~nm}$. (B) Binding isotherm fit to the observed data with inset showing the agreement between the observed and the calculated experimental data.

Figure 5.7. ELISA plot of microcystin LR binding, corresponding to a decrease in enzymatic activity as microcystin LR concentrations were increased from $1 \times 10^{-11}$ to $5 \times 10^{-9} \mathrm{M}$ (grey line) and the concurrent interference in the binding profile produced by the presence of the free antibody ( $1 \times 10^{-9} \mathrm{M}$, black line).

Figure 5.8. ELISA plot of microcystin LR binding, corresponding to a decrease in enzymatic activity as microcystin LR concentrations were increased from $1 \times 10^{-11}$ to $5 \times 10^{-9} \mathrm{M}$ (grey line) and the concurrent interference in the binding profile produced by the presence of the $\mathrm{H} 18$ aptamer $\left(1 \times 10^{-6} \mathrm{M}\right.$, black line $)$.

Figure 5.9. ELISA plot of microcystin LR binding, corresponding to a decrease in enzymatic activity as microcystin LR concentrations were increased from $1 \times 10^{-11}$ to $5 \times 10^{-9} \mathrm{M}$ (grey line) and the concurrent interference in the binding profile produced by the presence of the $\mathrm{H} 12$ aptamer $\left(1 \times 10^{-6} \mathrm{M}\right.$, black line) 163

Figure 5.10. ELISA plot of microcystin LR binding, corresponding to a decrease in enzymatic activity as microcystin LR concentrations were increased from $1 \times 10^{-11}$ to $5 \times 10^{-9} \mathrm{M}$ (grey line) and the concurrent interference in the binding profile produced by the presence of the LRL3 aptamer $\left(1 \times 10^{-6} \mathrm{M}\right.$, black line). 164

Figure 5.11. SPR angle variation versus $\mathrm{H} 12$ aptamer concentration resulting in a dissociation constant of $1.5 \mu \mathrm{M}$. 166

\section{Chapter 6}

Figure 6.1. Human kallikrein 6 with the catalytic triad of the active site highlighted in yellow and the inhibitor benzamidine (red) which is known to bind the $\$ 1$ pocket of the enzyme. This figure 
was generated from the using pymol (Delano 2002) and the crystal structure deposited by Bernett et al., (2002) in the protein data base (PDB ID: 1L2E).

Figure 6.2. Schematic for the selection of aptamers which inhibit kallikrein activity involving a four step procedure. A) Initially the DNA pool was mixed with kallikrein followed by B) washing away of unbound sequences through a filter which prevented loss of the enzyme. C) The enzyme sample was then exposed to competitive selection on a benzamidine column to separate sequences which bound the active site of the enzyme from those that bound other sites of the enzyme. D) Isolated sequences were amplified with PCR and analysis for activity modulation

Figure 6.3. Kallikrein activity in the presence of different concentrations of the pools isolated from the first round of selection: A) Sample7 and B) Sample8-11.

Figure 6.4. Kallikrein activity in the presence of different concentrations of the pools isolated from the second round of selection: A) Sample7 and B) Sample8-11. 187

Figure 6.5. Kallikrein activity in the presence of different concentrations of the pools isolated from the third round of selection. Sample derived from fraction 7 of the first round of selection.

Figure 6.6. Kallikrein activity in the presence of different concentrations of the pools isolated from the fourth round of selection of sample7.

Figure 6.7. Kallikrein activity in the presence of different concentrations of the pools isolated from the third selection round of sample8-11. 190 Figure 6.8. Observed change in kallikrein activity associated with elution the first 8 fractions produced from the third round for sample ${ }^{3}$ where fractions were eluted from the enzyme sample.

\section{Appendix}

Appx Figure 1. Elution of the DNA homolog of the RNA B12 aptamer from the B12 column. The DNA was eluted in $1.5 \mathrm{~mL}$ fractions from the column using $3.5 \mathrm{M}$ urea, $90^{\circ} \mathrm{C}$.

Appx Figure 2. Deposition of 6-Mercaptohexanol on the gold electrode pad of the QCM crystal. Decrease in frequency at which the QCM crystal vibrated was used to monitor the deposition of the organic monolayer from a $0.1 \mathrm{M} 6$-mercaptohexanol solution in buffer. 222 Appx Figure 3. Quartz crystal microbalance study of the potential binding between the aptamer and dopamine on the 6-Mercaptohexanol backfilled QCM crystal. The frequency of the aptamer functionalized crystal was monitored as increasing concentrations of dopamine were introduced to the flow cell $(0,0.87$ and $870 \mu \mathrm{M})$.

Appx Figure 4. Repeat of the isothermal titration microcalorimetric (a) raw data for the analysis of potential dopamine and aptamer interactions using thirty $6 \mu l$ injections of $0.47 \mathrm{mM}$ dopamine into $1.4 \mathrm{mLs}$ of $20 \mu \mathrm{M}$ dopamine aptamer and (b) the associated integrated heat plot. 224 
Appx Figure 5. Repeat of the isothermal titration microcalorimetric with ascorbic acid omitted from the buffer (a) raw data for the analysis of potential dopamine and aptamer interactions using thirty $6 \mu \mathrm{l}$ injections of $0.47 \mathrm{mM}$ dopamine into $1.4 \mathrm{mLs}$ of $20 \mu \mathrm{M}$ dopamine aptamer and (b) the associated integrated heat plot.

Appx Figure 6. Fluorescein labelled DNA homologue of the RNA Dopamine aptamer (4 $\mu \mathrm{M})$ run on A) a continuous gradient gel from 12-19\% acrylamide gel and $\mathrm{B}_{3}$ ) a $19 \%$ gel at a constant 35 mAmps for 21 hours. Lane 1 and 2 contain just the aptamer while lane 3 and 4 were run in the presence of $10 \mathrm{mM}$ dopamine to ensure the presence of the dopamine bound conformation. The relative fluorescence of the fluorescein labelled aptamer was examined with an Alphalmager EC System equip with a $537 / 35 \mathrm{~nm}$ filter. 226

Appx Figure 7. Fluorescein labelled DNA homologue of the RNA Dopamine aptamer $(4 \mu \mathrm{M})$ run on an $8 \%$ acrylamide gel at a constant 45 mAmps for 2 hours. Lane 1 and 2 contain just the aptamer while lane 3 and 4 were run in the presence of $100 \mathrm{mM}$ dopamine to ensure the presence of the dopamine bound conformation. The relative fluorescence of the fluorescein labelled aptamer was examined with an Alphalmager EC System equip with a 537/35 nm filter:

Appx Figure 8. Fluorescent anisotropy binding curve for the DNA form of the RNA dopamine aptamer and norepinephrine. Anisotropy values were collected using 0.8 EM norepinephrine which was exposed to increasing concentrations of the aptamer $(0.27,2.7,27,270,2700,27000$ $\mathrm{nM})$. The inset shows the agreement between the observed and the calculated anisotropy values. 228

Appx Figure 9. Fluorescent anisotropy binding curve for the DNA form of the RNA dopamine aptamer and tyramine. Anisotropy values were collected using $1 \mu \mathrm{M}$ tyramine which was exposed to increasing concentrations of the aptamer $(0.27,2.7,27,270 \mathrm{nM})$. The inset shows the agreement between the observed and the calculated anisotropy values. 


\section{List of Tables}

\section{Chapter 2}

Table 2.1. DNA sequences used to characterize the binding properties of the DNA homolog of the RNA dopamine aptamer. RNA1: original RNA sequence (Mannironi, et al., 1997).

\section{Chapter 3}

Table 3.1. Specificity characterization of the aptamer functionalized glass carbon electrode .. 119

\section{Chapter 4}

Table 4.1. SELEX identified aptamer sequences with potential secondary structures predicted using the RNAfold suit of the Vienna RNA Websuite (Gruber et al., 2008) and experimentally determined dopamine binding constants. The bracketed number under their name indicates the prevalence of each sequence during sequencing. 135

\section{Chapter 5}

Table 5.1. Microcystin LR binding affinities determined using anisotropy and circular dichroism for sequences generated from the microcystin LR SELEX. 
Chapter 1: DNA as a programmable nanomaterial 


\section{Aptamers, using natural polymers as nano receptors}

Aptamers are a novel class of nanomolecular receptors based on the biological building blocks of DNA, RNA and more recently peptides (Jayasena, 1999; Crawford et al., 2003). By taking advantage of the inherent complexity of these biological polymers, aptamers allow for a new way of producing programmable manipulations on the nanoscale. The term aptamer derives from the Latin word aptus meaning "to fit" or "fitted" (Ellington and Szostak, 1990) which alludes to their function as high affinity binding molecules. Akin to antibodies, aptamers are molecules that are able to selectively bind targets with high specificity and affinity. As such they have been developed for use as molecular tags and biosensors in both qualitative and quantitative detection technologies (Chen et al., 2011; Soontornworajit and Wang, 2011). The utility of aptamer-based technologies hold the promise of supplanting antibody-based detection technologies for several reasons. While aptamers are composed of biological building blocks, they are derived from an in vitro selective process known as Systematic Evolution of Ligands by EXponential enrichment (SELEX), rather than through in vivo immunogenic production and isolation processes characteristic of antibody-based technologies. This allows for several advantages over techniques used in the generation of antibodies such as the ability to generate binders to molecules which are nonimmunogenic, hazardous or toxic to organisms used to generate antibodies and the abilities to select for high affinity molecules in conditions other than physiological (Stoltenburg et al., 2007). This flexibility in the conditions used to generate aptamers allows for the incorporation of aptamers in wider application than can be achieved with 
antibodies and the customizable matching of selection conditions with the conditions anticipated for potential applications (Stoltenburg et al., 2007).

The process for producing aptamers, SELEX, relies on the exposure of an initial large pool of randomly generated sequences to a target of interest. These pools are designed to contain random regions usually between 20 and 100 nucleotides in length and can be composed of up to $10^{15}$ distinct nucleotide sequences (Stoltenburg et al., 2007). The process itself (Figure 1.1) involves the exposure of this pool to the target of interest which is usually sequestered to a column. Once the nonbinding sequences are washed away the sequences of interest are isolated and amplified using PCR such that a new pool with sequences of varying affinity to the target are generated. This new pool is then used to sort the candidate sequences based on their affinity for the target in a second round of exposure to the target molecule. This process continues for several rounds until the generated pool contains primarily high affinity target binding sequences that are isolated and characterized (Stoltenburg et al., 2007). This process has allowed for the production of aptamer-based detection systems able to detect thousands of biologically important markers, a technology that will revolutionizing the medical field as it is brought to market (Gold et al., 2010).

However, the development of aptamer based technologies has encountered a variety of problems during its inception, ranging from low rates in successful aptamer production from SELEX (McKeague and DeRosa 2012) to difficulties in determining the minimal binding sequences required for target interactions, which would aid in the incorporation of aptamers into microarrays (Fischer and Tarasow 2011). The determination of binding affinities for 
aptamers of small molecules has also been problematic resulting in uncertainty and loss of confidence in values reported in the literature (McKeague and DeRosa 2012). While there may be difficulties associated with determining binding affinities, the successful incorporation of aptamers such as those for ATP (Huizenga and Szostak 1995) and cocaine (Mascini et al., 2012) into biosensors illustrate the potential of the technology.

Aptamers have been produced using both amino acids and nucleotides, due to the sequential and programmable nature of both of these types of molecules. Peptides and RNA can be rationalized as ideal materials for aptamer production based on the tertiarystructures they form in the performance of their biological functions. Amino acid based enzymes and proteins provide enough molecular diversity to support the majority of cellular processes (Lichtarge and Wilkins. 2010). RNA, while existing primarily as a short lived cellular messenger system, also assumes complex tertiary structures that are biologically active, such as riboswitches (Wachter, 2010), ribosomes (Agirrezabala and Frank, 2010) and ribozymes (Zhang et al., 2010). This cross over between information system and functionally active biological molecules has contributed to the theory that RNA functionality supported early life (Joyce, 2009). 


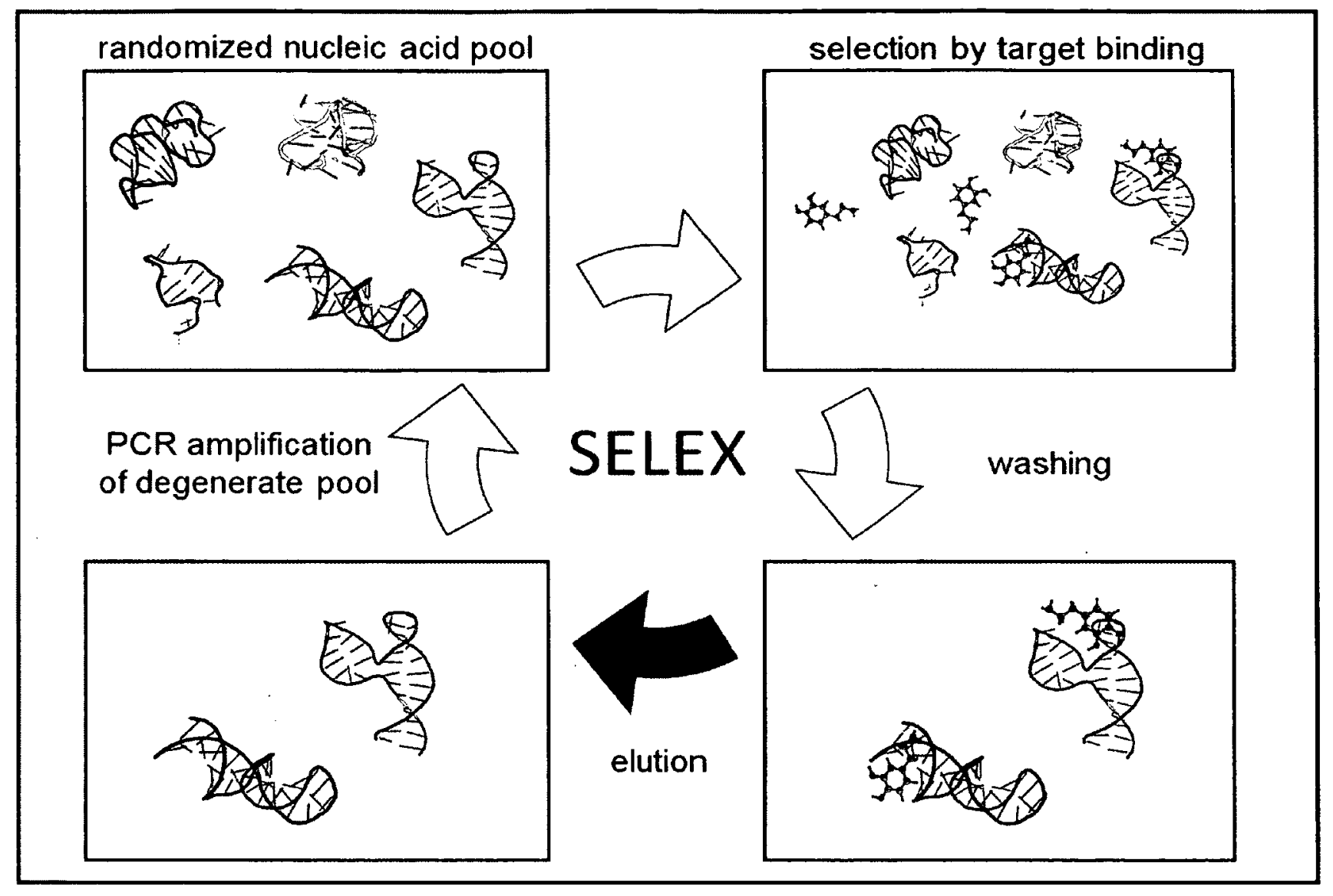

Figure 1.1. Schematic representation of the SELEX process. Initially the pool of random sequences is exposed to a target of interest, followed by washing away of sequences with no low affinity for the target. The sequences of interest are then separated from the target and amplified to produce a new pool. This new pool is then reintroduces to the target to sort higher affinity sequences from lower affinity sequences. This cycle is repeated multiple times to enrich the number of high affinity sequences present before a sample of the pool is sequenced to determine the primary aptamer sequences which can be characterized by their binding affinity for the target. 
However, the lack of natural structural variety associated with DNA raises the question as to whether DNA is able to mimic the structural diversity associated with its daughter molecule RNA or whether any structure complexity associated with single stranded DNA will be distinct from similar sequences produced by RNA.

\section{Double stranded DNA}

Biologically, DNA in its in vivo form is typically found as a long double stranded duplex, which only presents variable binding surfaces along the major and minor grooves of the helix (Brown and Hurley, 2011). While these differences do provide enough variability to allow transcriptional regulatory protein to bind in controlled fashions, this simple tertiary structure limits the ability of the double stranded DNA itself to act as a recognition element for much more than its complementary sequence. Sequence complement, however, is a very highly specific and high affinity binding process which has been exploited with DNA based nanotechnology, through the production of DNA based molecular machines (Liu and Liu, 2009; Lee and DeRosa 2010) and complex scaffold architectures created with DNA origami (Kuzuya and Komiyama, 2010; Figure 1.2). 


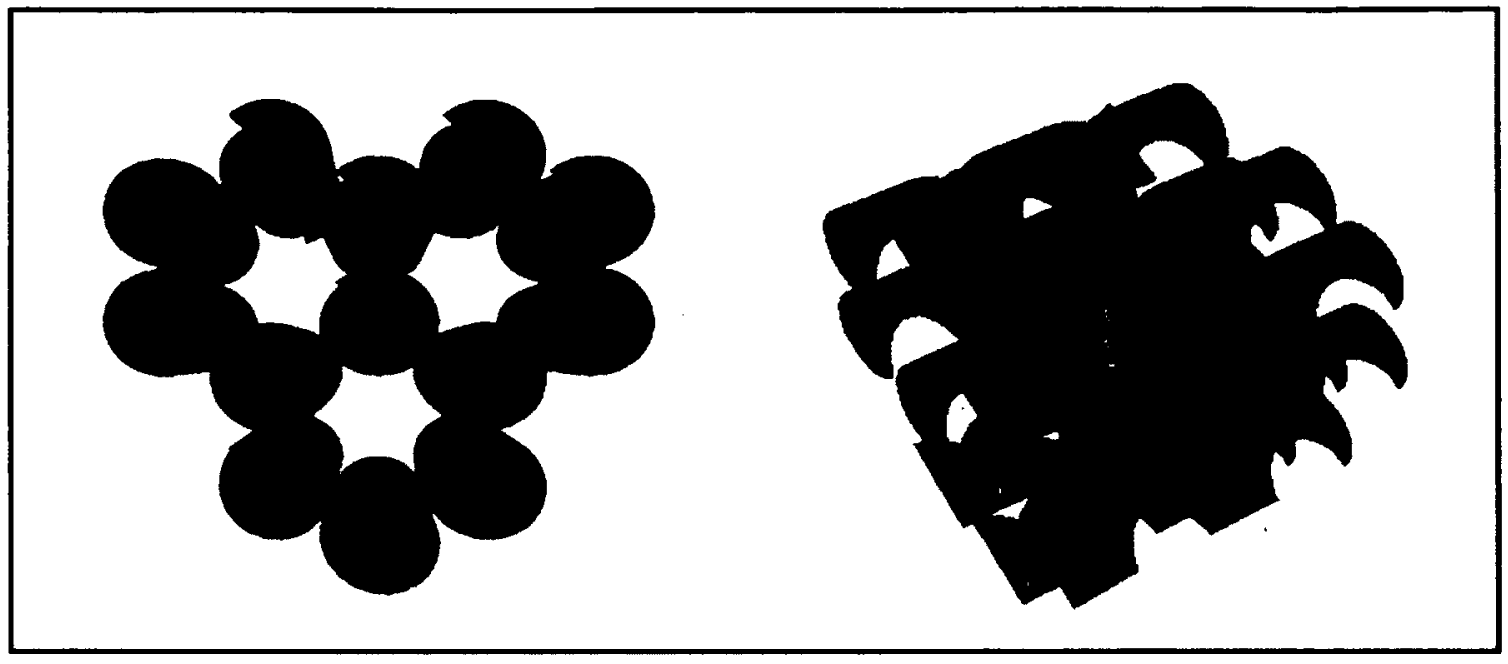

A

1

Figure 1.2. Simple scaffold structure generated with CADNANO a program for templating DNA origami structures (Douglas et al., 2009,'http://cadnano.org/).

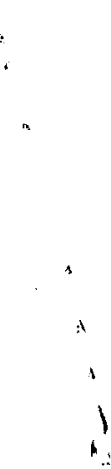


The double stranded helical structure of DNA has been shown to be permissive to changes in its coiling, resulting in multiple alternative helical forms (Figure 1.3). This is in contrast to helical double stranded RNA sequences which is confined primarily to the A helical conformation due to the repulsion between the 2 'hydroxyl group located on the ribose of RNA and the phosphate backbone (Salazar et al., 1993). Twisting of the DNA helix can shift the helix away from B DNA, its native relaxed state, to more tightly coiled forms associated with the supercoiling of chromosomal DNA or to its more loosely coiled form A DNA. A DNA changes the surface exposure of double stranded DNA, widening the minor grove and narrowing the major grove. This change in structure is believed to be associated with increased binding specificity between DNA and proteins involved in its expression, regulation and processing (Lu et al., 2000). 


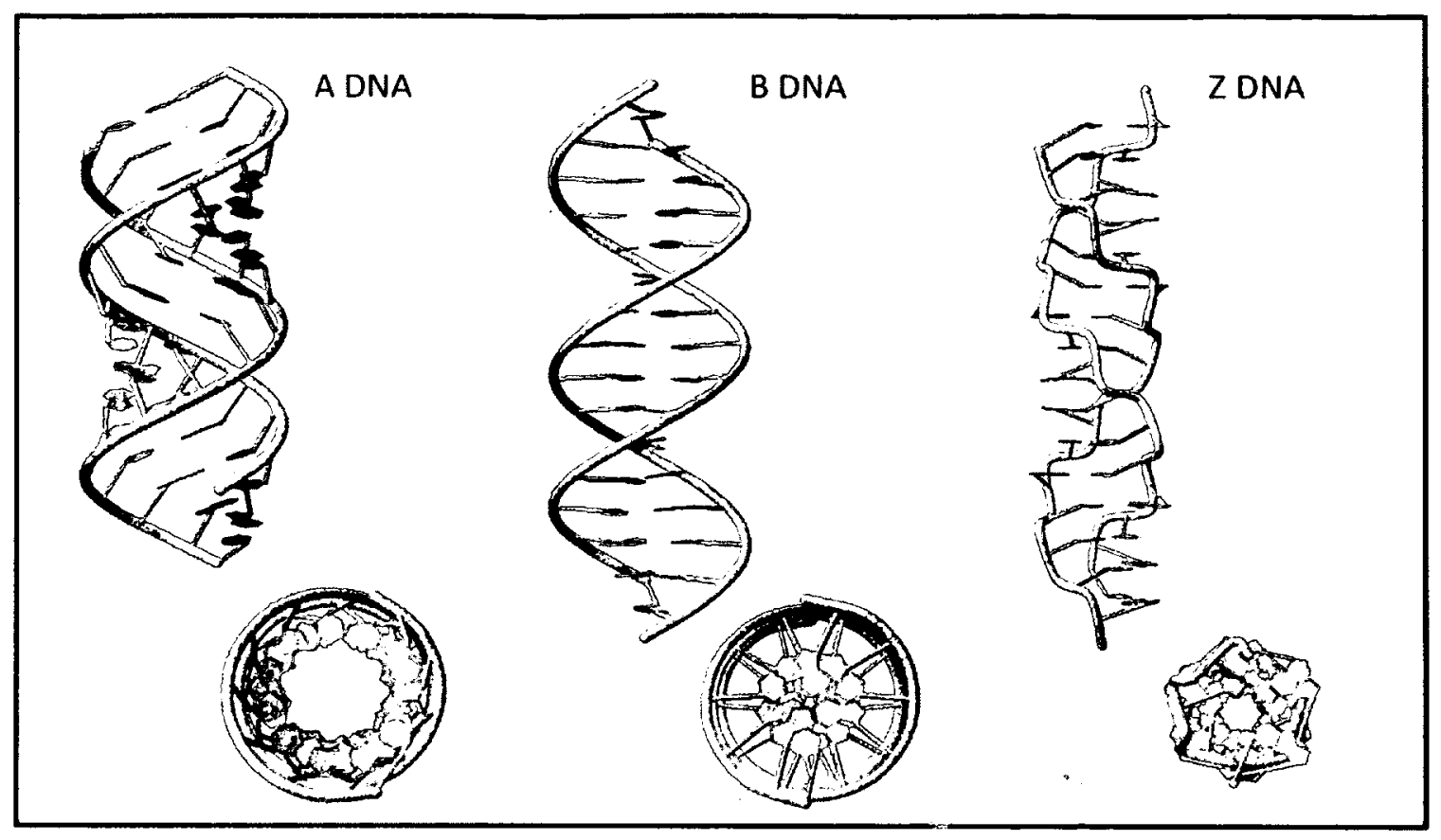

Figure 1.3. Known variations of the DNA double helix A) A DNA is very similar to the coiling produced by RNA, B) B DNA is the most common helical structure with a positive twist of $36^{\circ}$ per base pair (Lu and Olson, 2008). C) The more unusual Z DNA has been found in regions of high GC content and has a negativ a twist of $-60^{\circ}$ per base pair (Lu and Olson, 2008). This figure was generated with Web 3DNA (Zheng et al., 2009; http://w3dna.rutgers.edu) and Jmol (Herráez, 2006; Jmol: an open-source Java viewer for chemical structures in 3D. http://www.jmol.org//. Models were produced using the DNA sequence atcgatcgatcgatcg for $A$ and $B$ DNA while eight CG repeats were used to generate the $Z$ DNA construct. 
The hydrogen bonding that holds the helix together form the basis of the sequence specific binding of complementary DNA strands (Figure 1.4). Helical DNA relies on the specific base pairing of adenine to thymine and guanine to cytosine, the former containing two hydrogen bonds and the latter containing three. The order that these bases appear in the DNA sequence provides extreme specificity to the binding between complementary DNA strands. It also provides huge affinity between the two complementary strands as conventional binding theory suggests that three point binding is a prerequisite for high affinity binding (Ogston, 1948), while complementary DNA strands have a minimum of four hydrogen bonds every tv'o base pairs. This high density of hydrogen bonding between complementary strands produces such high binding affinity between complementary strands that the melt temperature required to disrupt the hydrogen bonding denaturing the double stranded DNA strands becomes a more useful measure of binding affinity between complementary strands. 

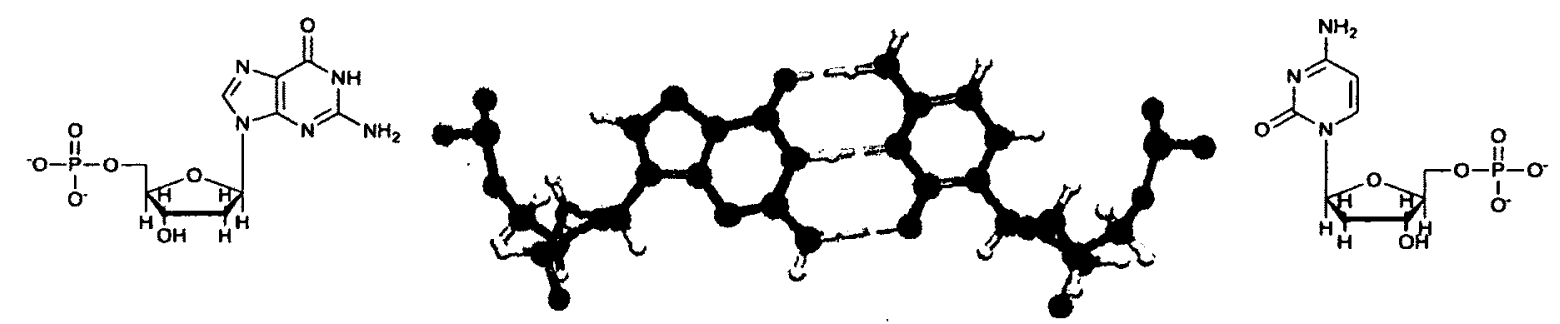

Guanine (G)

Thymine (T)

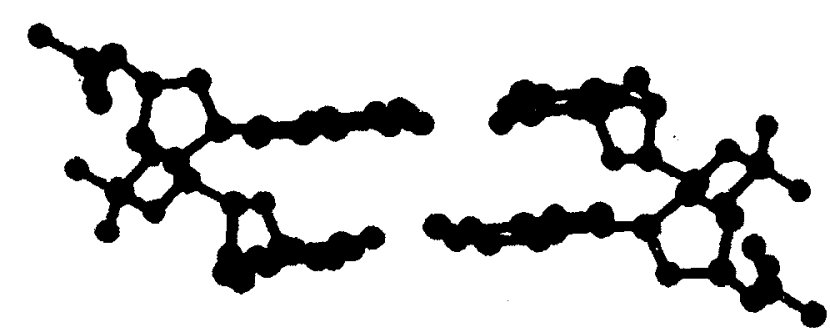

Cytosine (C)

Adenine (A)
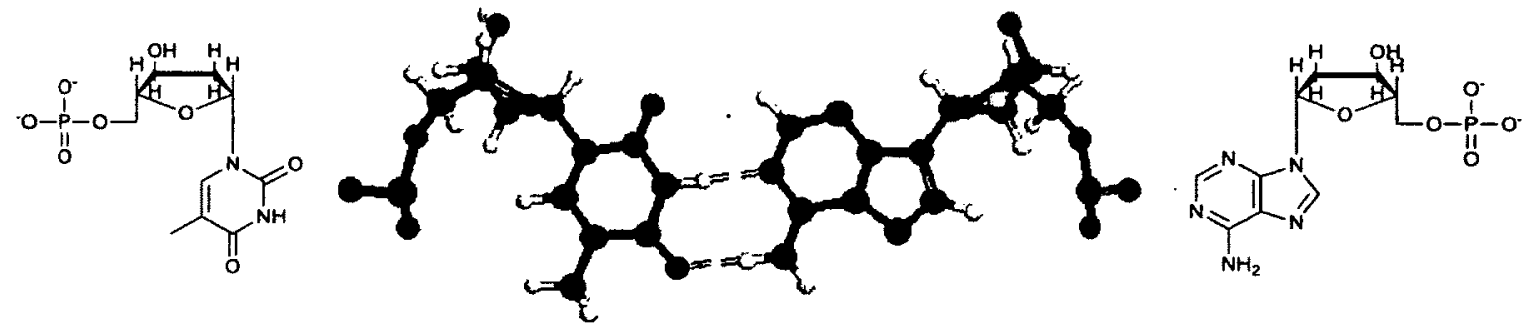

Figure 1.4. Structure of deoxyribonucleic acid (DNA) depicting the two purines, adenine (A) and guanine (G), base pairing to their respective partner pyrimidines, thymine (T) and Cytosine (C). This Watson-Crick binding forms the basis for DNA sequence complement binding which produces the double stranded helix. Figure generated with Web 3DNA (Zheng et al., 2009; http://w3dna.rutgers.edu) and Jmol (Herráez, 2006; Imol: an open-source Java viewer for chemical structures in 3D. http://www.jmol.org/). 


\section{Single stranded DNA}

In contrast to the rigidity observed with double stranded DNA, single stranded DNA is very similar to RNA in its propensity to form complex tertiary structures which are dependent on its primary sequence (Brown and Hurley, 2011). Single stranded DNA has been found to produce structures such as steam loop, pseudo knots, triplexes (Htun and Dahlberg 1989) and g-quadruplexes both in vivo (Sun and Hurley, 2009) and in vitro (Brown and Hurley, 2011; Figure 1.5). The formation of these structures results from both Watson-Crick and non-Watson-Crick base pairing that has been observed in RNA as well (Lavery et al., 1992; Leontis et al., 2002). Non-Watson-Crick base pairing can result from hydrogen bonding of the Watson-Crick edge with the Hoogsteen or sugar edge or not involve the Watson-Crick edge at all (Figure 1.6). While Watson-Crick base pairing is by far the most common interaction stabilizing nucleotide contacts and is used to define secondary structures, non-Watson-Crick binding has been implicated as important in formation of tertiary single stranded nucleic acid structures. In particular, naturally occurring RNA structures are known to contain nucleotides which are conserved suggesting the importance of non-Watson-Crick binding in tertiary structure formation (Lavery et al., 1992). Similarly, DNA structures such as g-quadruplexes (Figure 1.5) which stabilize chromosomal telomeres and are fairly common in aptamer studies, also exemplify the importance of non-Watson-Crick binding stabilization in non-helical nucleotide structures (Brown and Hurley, 2011). 
A

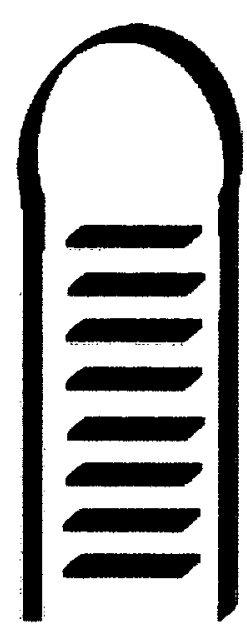

C

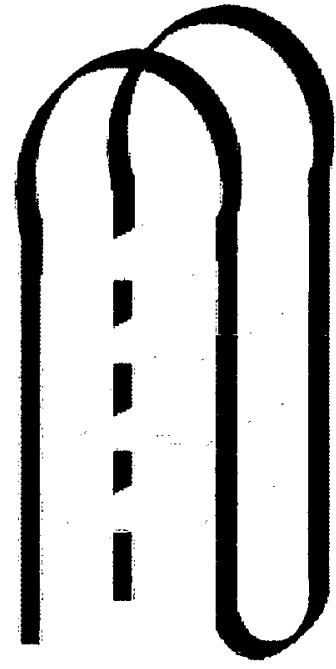

B

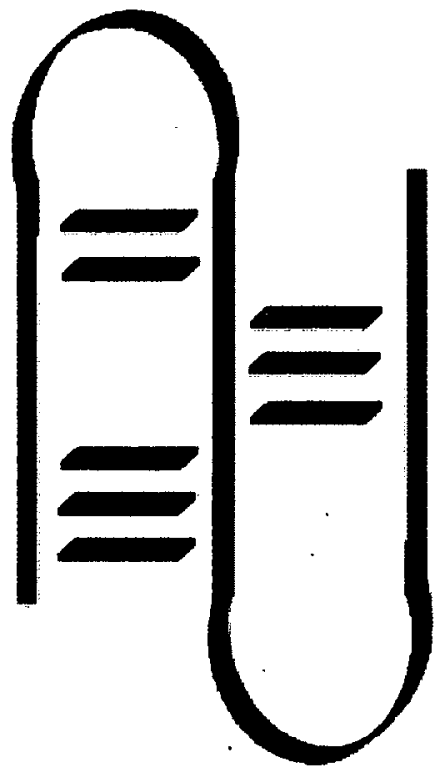

D<smiles>Cn1cnc2c(=O)[nH]c(N)nc21</smiles><smiles>Cn1cnc2c(=O)[nH]c(N)nc21</smiles>
$\mathrm{K}^{+}$<smiles>Cn1cnc2c(=O)[nH]c(N)nc21</smiles><smiles>Cn1cnc2c(=O)[nH]c(N)nc21</smiles>

Figure 1. 5. Schematic representation of some of the tertiary structures produced by single stranded DNA. The hairpin (A) is produced by looping back of a single strand while more complex structures such as pseudo knots (B) can occur when base pairing involves multiple looping events. These structures are not constrained by Watson-Crick base pairing associated with the double helix and may result in even more complex base pairing such as is observed with g-quadraplexes for their stability (C-D) (Parkinson, 2006). 


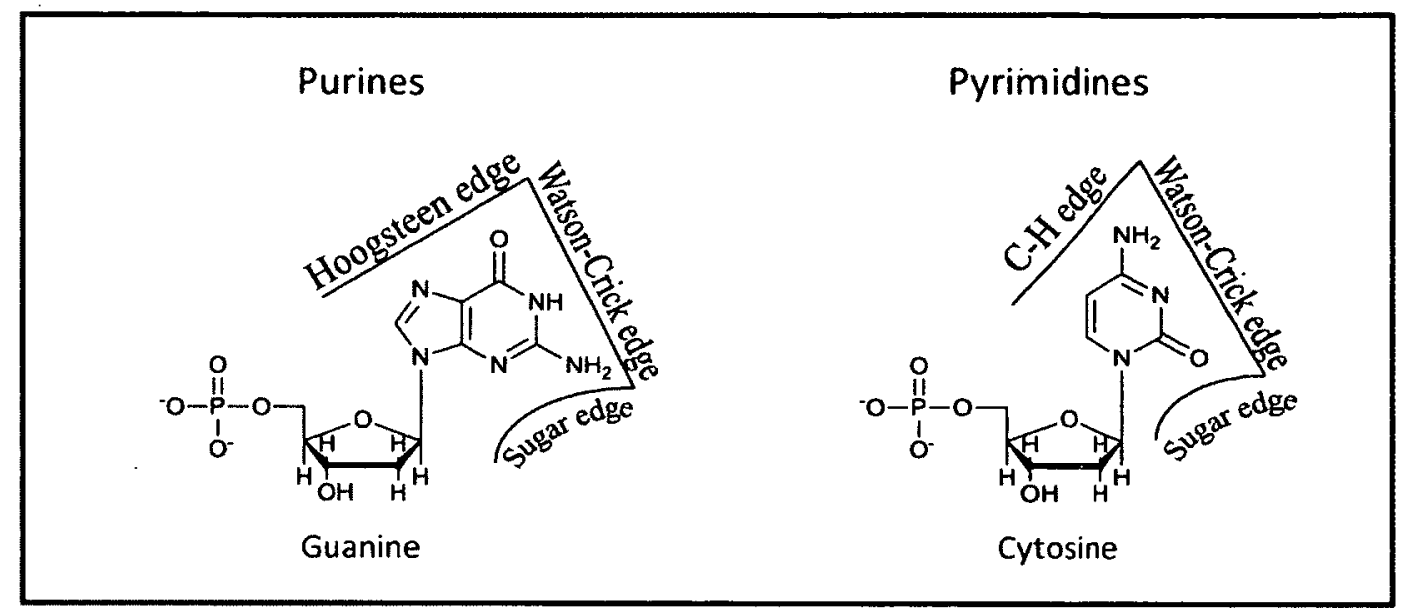

Figure 1. 6. Schematic of the edges used to designate the interactions between nucleobases. Each base has a Watson-Crick edge and a sugar edge. The difference in structure between the purine bases and pyrimidine bases is distinguished with the designation of the Hoogsteen edge and the $\mathrm{C}-\mathrm{H}$ edge (Leontis et al., 2002).

The ability of DNA to fold in this way allows the formation of tertiary structures that can produce the complex surfaces required for three point binding (Ogston, 1948) a prerequisite for high specificity ligand recognition (Gold et al., 1995; Hermann and Patel, 2000). SELEX has been used to exploit this ability with the use of large random pools of DNA, up to $1 \times 10^{15}$ individual sequences, the large diversity of these pools has allowed for the identification ofDNA: aptamers with similar target specificity to RNA aptamers 
produced in similar manners. However, the resulting sequences and structures have been found to be distinct from those produced with RNA (Silverman, 2009). Although the difference in observed sequence between aptamers produced with either RNA or DNA may result from the large number of possible sequences associated with the selection space, rather than a lack in the ability of DNA to mimic the structures produced by RNA.

The ability of DNA to mimic the structure associated with RNA would expand the application of nucleotides to nanotechnological applications for several reasons. DNA is known to be more stable than RNA as RNA is more susceptibility to auto cleavage of its phosphate backbone mediated by phosphoester transfer to the 2'hydroxyl group of the ribose (Mok and Li, 2008). Under normal physiological conditions the half-life for RNA phosphoester hydrolysis has been extrapolated to be approximately 100 years while the comparable half life for the hydrolysis of the DNA phosphoester is approximated to 31 million years (Wolfenden, 2011). RNA is also a highly regulated transient biological signalling molecule that is primarily regulated through degradation so ribonucleases are quite abundant in nature (Deutscher, 1993) while DNA's role as a long term information storage structure means that there are limited deoxyribonucleases and their activity is highly regulated (Walther and Edmunds, 1970).

The evolutionary biological role of RNA as a multipurpose information signalling and catalytically active molecule has resulted in the natural world producing countless functional biologically structures such as riboswitches (Wachter, 2010) and ribozymes 
(Zhang et al., 2010) which if the stability could be improved would allow for their integration into the nucleotide based nanotechnological tool box.

\section{DNA based Nanoapplications}

The high affinity of DNA for its complement has revolutionized bioinformatics with the use of single stranded DNA microarrays that, in combination with whole genome sequencing, has allowed determination of RNA expression profiles, genetic risk factor disease correlation and rapid genetic disease detection (Yoo et al., 2009). Additionally, double stranded DNA chips have allowed for rapid determination of binding sequence specificity for proteins that regulate or bind to DNA (Bulyk, 2006). However, the unique characteristic of DNA has rapidly expanded its use beyond bioinformatics into the realm of an all-purpose programmable nanomaterial. For example, DNA has been used to produce regular repeating scaffold-like structures (Kuzuya and Komiyama, 2010), three dimensional functional nanostructures (Kuzuya and Komiyama, 2010), simple machines and motors (Lee and DeRosa 2010; Kang, 2009; Liu and Liu, 2009) and aptamers, high affinity ligand binders (Ellington and Szostak, 1990). In particular, paralleling the initial development of DNA microchips, aptamer microchip arrays are allowing for whole cell or serum monitoring of protein expression levels in a technology that may rival mass spec techniques (Gold et al., 2010; Baird et al., 2011; Baldrich, 2011). Additionally, the high affinity binding properties of aptamers have not been solely exploited for medical diagnostics but have also been used as biosensors in the detection of a variety of ligands 
(Cho et al., 2009) including inorganic toxins (Lin et al., 2011), production of signal triggered self assembly (Wang 2009), been incorporated into targeted delivery systems (Wu et al., 2010), systems for triggered release (Sultan and DeRosa, 2011) and been used as therapeutics in their own right (Esposito et al., 2011).

While still in the early stages, the adaptation of DNA-based materials to a variety of fields holds the promise of revolutionizing our control and perception of things on the nanoscale.

\section{DNA as a multifunctional nanomaterial in biological applications}

The superior biological stability of DNA (Wolfenden, 2011), its non-immunogenic characteristics (Esposito et al., 2011) and the development of tools for the predictive structural production of DNA based scaffold (Kuzuya and Komiyama, 2010) make DNA an attractive material for nanotechnological applications: Towards this goal of improving the applicability of DNA in nanotechnological applications, this work describes the characterization of DNA homologues of the known RNA aptamers for dopamine (Walsh and DeRosa 2009) and vitamin B12. This work also details the application of the DNA homolog of the RNA dopamine aptamer to the electrochemical detection of dopamine. Additional chapters detail the use of SELEX for the production of DNA aptamers. These studies examine the production of de novo DNA aptamers to dopamine, providing further evidence of DNA's equivalence to RNA as a programmable structural material: 
Alternative projects described in this work examine the production of DNA aptamers for the potential hepatotoxic environmental pollutant microcystin and the ability of DNA aptamers to modulate enzymatic activity The kallikrein study preliminarily suggests that SELEX-like selection can be used to generate aptamers that affect enzymatic activity both positively and negatively. As most therapeutics for metabolic processes are based on inhibitory approaches (Espiosito et al., 2011), the ability to tune aptamer selection to desired effect has the potential to add new avenues for pharmacological investigations into therapeutic treatment strategies. Additional support for the potential therapeutic use of DNA aptamers is highlighted with the application of the DNA homolog of the RNA dopamine aptamer to an in vivo pseudodialysis of dopamine, sequestering the neurotransmitter and reducing neuronal over activity (Holahan, et al., 2011).

\section{Thesis objectives}

The overall goal of this thesis was the assessment of DNA as a functional material for the production of aptamer based devices. To accomplish this several projects were undertaken:

DNA was directly compared with RNA to assess its ability to mimic the use of RNA in aptamer based technologies through the production of DNA homologs of the RNA aptamers for dopamine and B12. 
Using the DNA homolog of the RNA dopamine aptamer new methods for the fabrication of electrochemical DNA based aptamer sensors for dopamine were examined.

SELEX based projects were undertaken to examine the ability of DNA to produce high affinity binders for dopamine and microcystin LR.

Finally a SELEX based selection was also used to determine if selection methods could be used to produce DNA aptamers which could modulate catalytic activity of enzymes using kallikrein as the model enzyme target. 


\section{Chapter 2: Retention of function in the DNA homolog of the RNA dopamine aptamer}

Portions of this chapter have been published in:

Ryan Walsh, Maria C. DeRosa (2009) Retention of function in the DNA homolog of the RNA dopamine aptamer. Biochemical and Biophysical Research Communications $388: 732-735$ 


\section{Abstract}

While it is generally accepted that the functional tertiary structures formed by RNA cannot be replicated by a deoxy version of the same sequence, here we demonstrate that in some cases conservation of function can occur, illustrated with the DNA homolog of the dopamine RNA aptamer. Through affinity chromatography experiments we were able to initially demonstrate that the DNA homolog of the dopamine aptamer did retain its ability to bind this neurotransmitter. Further studies using gel shift techniques and ITC provide evidence that the tertiary conformation of the aptamer is not altered upon dopamine binding. Fluorescence anisotropy demonstrated that the DNA homolog binds dopamine with improved affinity and similar specificity relative to the RNA aptamer. Alterations to the primary sequence of the DNA homologue suggested that the way in which the DNA homologue bound dopamine was maintained in the transition from RNA. Further studies of the DNA homolog of the B12 RNA aptamer suggested that the more complex pseudo-knot architecture of this aptamer was not conducive to retention of the structure. These findings suggest that there may be a way to predict which sequences and tertiary structures may be good candidates for retention of function in conversions between both RNA and DNA. 


\section{Introduction}

DNA and RNA are very similar biological molecules, however their differences significantly contribute to their stability and function in biological processes. Differing primarily in a single nucleotide substitution which replaces thymine in DNA with uracil in RNA, both long polymers can form base pairs, double helical structures and more complex single stranded tertiary folding patterns (Linak et al., 2011; Brown and Hurley, 2011). Additionally the 2 -hydroxyl in RNA distinguishes it from DNA forcing the ribose of RNA into a $3^{\prime}$-endo pucker reducing the ability of RNA to compress its helical structure confining it to the A form when base pairing (Salazar et al., 1993). This hydroxyl also significantly reduces the stability of RNA and may relate to its role as a short lived messenger molecule in biological systems (Deutscher, 1993; Mok and Li, 2008; Wolfenden, 2011). However, evolutionary processes have produced a multitude of functional RNA structures such as riboswitches, ribozymes and the ribosome which control intricate regulatory, signalling and metabolic processes (Wachter, 2010; Zhang et al., 2010). Comparatively, DNA while very similar to RNA, has not evolved the diversity of structures associated with RNA due to its role as the major information storage unit in life. However, the use of RNA and DNA in the production of aptamers has clearly demonstrated the ability of DNA to form structures functionally if not structurally equivalent to those of RNA (Silverman, 2009).

The lack of structurally and functionally equivalent RNA and DNA molecules may be attributed to the differences between the polymer structures, however, some examples do exist. Studies by Lauhon and Szostak indicate that the DNA homolog of a riboflavin 
RNA aptamer was able to produce the guanine quadruplex structure responsible for ligand interactions although the resulting binding affinity was decreased (Lauhon and Szostak, 1995). A similar transformation but from DNA to RNA was able to produce a functional ribozyme but also with decreased activity (Travascio et al., 1999). Guanine quadruplexes were primary structural features in both cases suggesting that guanine quadruplexes may provide sufficient stability to accommodate limited retention of function between RNA and DNA. Additional studies suggest that other secondary structures are unable to do this as demonstrated by the loss of binding affinity produced by the DNA homolog of the RNA ATP aptamer, which consists of a stem loop (Huizenga and Szostak, 1995; Dieckmann et al., 1997). Similarly interconversions of the R3C RNA ligase ribozyme, and the 10-18 RNA ligase deoxyribuzyme into their corresponding homologs destroyed their activity (Paul et al., 2008). Furthermore, DNA homologs of the hammerhead ribozyme and hairpin ribozymes have also failed to produce functional structures (Jeffries and Symons, 1989; Chowrira et al., 1993; Chartrand et al., 1995).

The stability of DNA in comparison to RNA can be attributed to its lack of the hydroxyl in the $2^{\prime}$ position. This difference makes it hoth chemically and biologically more stable than RNA (Deutscher, 1993; Mok and Li, 2008; Wolfenden, 2011). Therefore, conversion of RNA to DNA removing the hydroxyl provides a potentially facile way of improving the stability of RNA aptamers, riboswitches and ribozymes provided that the changes do not affect the overall functionality of the molecule. 


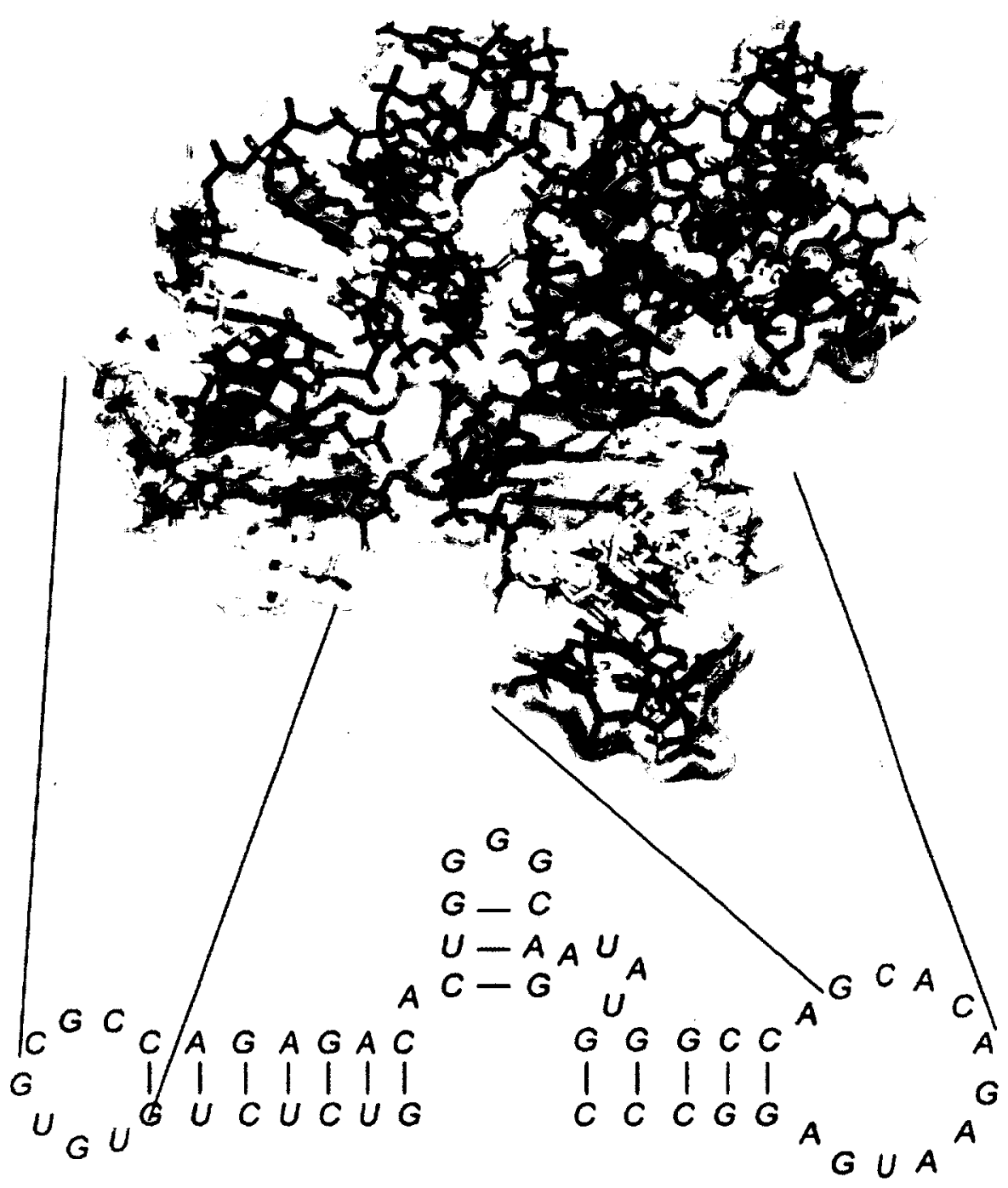

Figure 2.1. The secondary structure of the RNA dopamine aptamer proposed by Mannironi, et al., (1997) displayed with a tertiary structure of the aptamer generated using MC-Fold \& MC-Sym (Parisien and Major, 2008) and rendered using pymol (DeLano, 2002). Yellow highlighted regions correspond to the dopamine binding domain. Complementary and invariant bases in the loops that are required for dopamine binding are indicated in red and blue, respectively. 

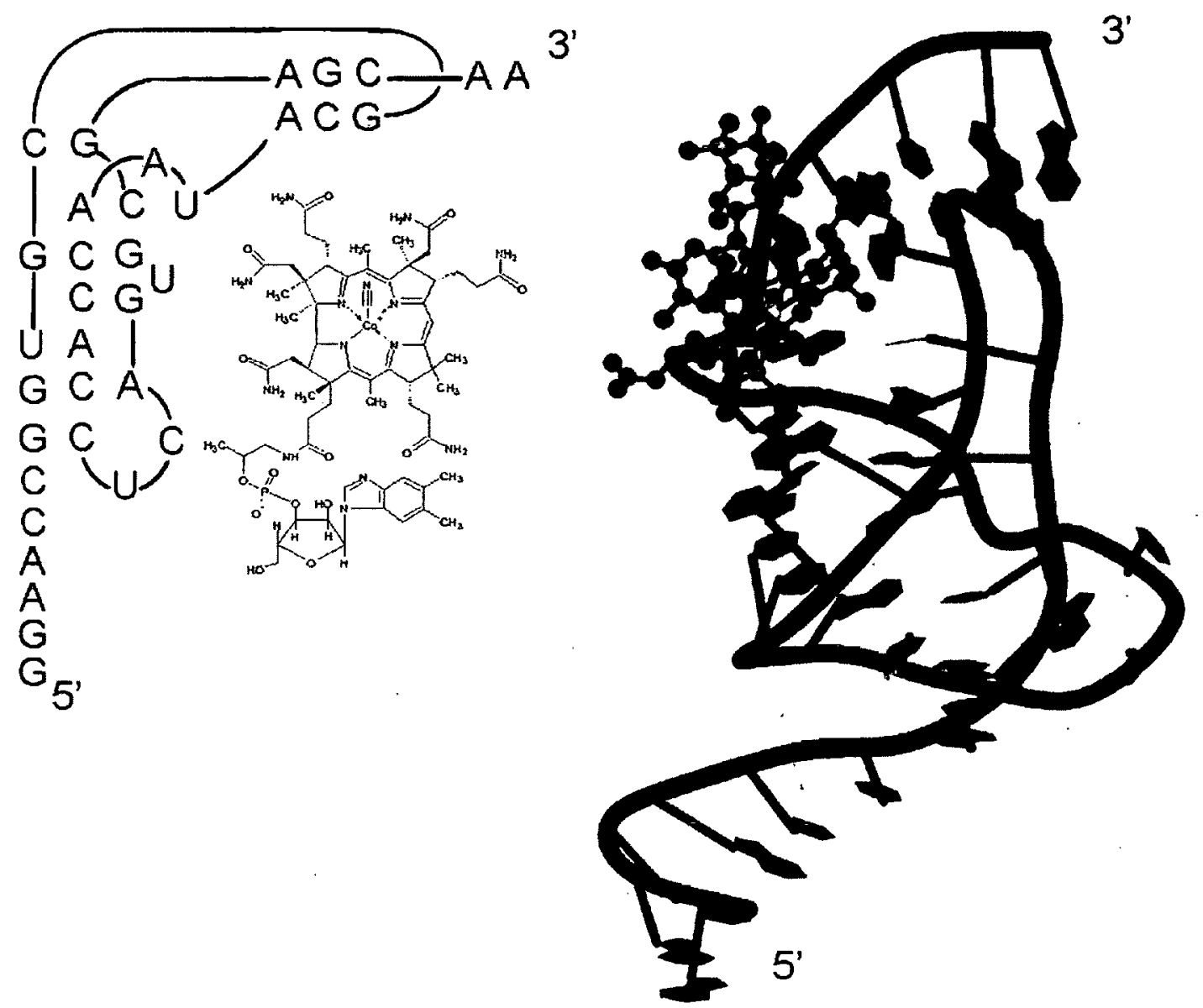

Figure 2.2. Schematic of the tertiary structure of the B12 RNA aptamer along with the crystal structure reported by Sussman, et al., (2000). The structure demonstrates the complex fold providing a complex surface which interacts with vitamin B12. The figure was generated using the modeling program Jmol (Herráez, 2006; Jmol: an open-source Java viewer for chemical structures in 3D. http://www.jmol.org/) and the crystal structure of the B12 aptamer complexed with B12, 1DDY (Sussman et al., 2000), in the protein Data bank (Berman et al., 2000). 
This work presents the conversion of the RNA dopamine aptamer (Figure 2.1; Mannironi et al., 1997), a double stem loop structure, into its DNA homolog. Additionally, a DNA homolog of the cyanocobalamin (vitamin B12) RNA aptamer (Figure 2.2; Lorsch and Szostak 1994; Sussman et al., 2000), a more complex pseudo knot RNA structure, is also examined for its functionality. To determine if the DNA homologs retain their affinity for their respective targets multiple experimental approaches were undertaken.

\section{Determination of binding affinities}

Aptamer target interactions, as with any receptor ligand system, adheres to the law of mass action. The development of receptor theory precedes but parallels that of the Langmuir equation (Colquhoun, 2006). The Langmuir equation was developed to describe the adsorption of molecules on a metal surface and describes the fractional adsorption relative to the total number of binding sites. While receptor binding theory follows the same logic, it relates to the total number of receptors in the system being studied. The law of mass action states that the fractional association, or reaction rate in the case of chemical reactions, is dependent on the concentration of the interacting species. As such, the mass action equations governing the interactions between aptamer and target can be derived using the assumption of a one to one interaction between the aptamer and target molecule (Equation 2.1). 


$$
A+T \underset{K_{\text {off }}}{\stackrel{K_{\text {on }}}{\longrightarrow}} A T
$$

For the derivation of the mass action relationship equation the following assumptions are required, all receptors in the system are equally accessible to the ligand, there is a one to one stoichiometry between receptor and ligand interactions, the formed complex does not change either ligand or receptor and that the interaction is completely reversible (Motulsky and Christopoulos, 2004). At equilibrium, the rate of aptamer target complex formation should equal the rate of the complexes dissociation (Equation 2.2) and produces an equation for the dissociation constant that is the same equation used to define equilibrium constants (Equation 2.3; Motulsky and Christopoulos, 2004; Bigott-Hennkens et al., 2008).

$$
K_{o n}[A][T]=K_{o f f}[A T]
$$

$$
\frac{[A][T]}{[A T]}=\frac{K_{\text {off }}}{K_{\text {on }}}=K_{d}=K_{e q}
$$

While this, in theory, is enough to determine the binding constant between the aptamer and the target, the practical problem of determining the concentrations of the species at equilibrium can make this challenging. That is, while the initial concentration 
of the aptamer and target may be known, the concentration they reach at equilibrium can be more difficult to determine (Equations $2.4 \& 2.5$ ).

$$
[A]=[A]_{o}-[A T]
$$

$$
[T]=[T]_{o}-[A T]
$$

Inserting these values into the equilibrium equation (Equations $2.6-2.10$ ) results in a second order polynomial based on the concentration of the aptamer-target complex ([AT]; Equation 2.10).

$$
\frac{\left([A]_{o}-[A T]\right)\left([T]_{o}-[A T]\right)}{[A T]}=K_{d}
$$

$$
\left([A]_{o}-[A T]\right)\left([T]_{o}-[A T]\right)=[A T] K_{d}
$$

$$
\left([A]_{o}-[A T]\right)\left([T]_{o}-[A T]\right)-[A T] K_{d}=0
$$

$$
[A]_{o}[T]_{o}-[A T][T]_{o}-[A T][A]_{o}+[A T]^{2}-[A T] K_{d}=0
$$




$$
[A T]^{2}-\left([T]_{o}+[A]_{o}+K_{d}\right)[A T]+[T]_{o}[A]_{o}=0
$$

This can be used to solve for the dissociation constant of the system using the quadratic equation (Equation 2.11).

$$
[A T]=\frac{\left([T]_{o}+[A]_{o}+K_{d}\right)-\sqrt{\left([T]_{o}+[A]_{o}+K_{d}\right)^{2}-4[T]_{o}[A]_{o}}}{2}
$$

While this can be used to define the binding affinity between an aptamer and its target, a more common way of determining the binding affinity involves the use of fractional occupancy (Motulsky and Christopoulos, 2004) which relates maximum binding $\left(B_{\max }\right)$, associated with the total number of receptors in the assay, to the dissociation constant $\left(K_{d}\right)$. In this case, the fractional occupancy can be defined as the concentration of the aptamer-target complex divided by the total aptamer concentration (Equation 2.12).

$$
\begin{gathered}
\text { fractional occupancy }=\frac{[A T]}{[A]_{0}}=\frac{[A T]}{[A]+[A T]} \\
{[A T]=\frac{[A][T]}{K_{d}}}
\end{gathered}
$$




$$
\begin{gathered}
\text { fractional occupancy }=\frac{\frac{[A][T]}{K_{d}}}{[A]+\frac{[A][T]}{K_{d}}} \\
\text { fractional occupancy }=\frac{[T]}{K_{d}+[T]}
\end{gathered}
$$

By defining the concentration of the aptamer-target complex in terms of the dissociation constant (Equation 2.3 rearranged to equation 2.13) the concentration of the receptor drops away (Equation 2.14) resulting in a fractional occupancy equation which is only dependant on the concentration of the target and the dissociation constant (Equation 2.15). As the concentration of the free target is not adjusted to account for the aptamer-target complex in this treatment of binding kinetics, the accuracy is higher when the change in the target concentration due to formation of the aptamer target concentration approaches zero. Due to the high degree of binding affinity ascribed to receptor-ligand interactions, most receptor ligand binding interactions can be defined using this equation as the receptor concentration generally is several orders of magnitude below the concentration of the ligand.

The fractional occupational relationship results in the characteristic rectangular hyperbola know as the binding isotherm (Figure 2.3; Motulsky and Christopoulos, 2004). 
A

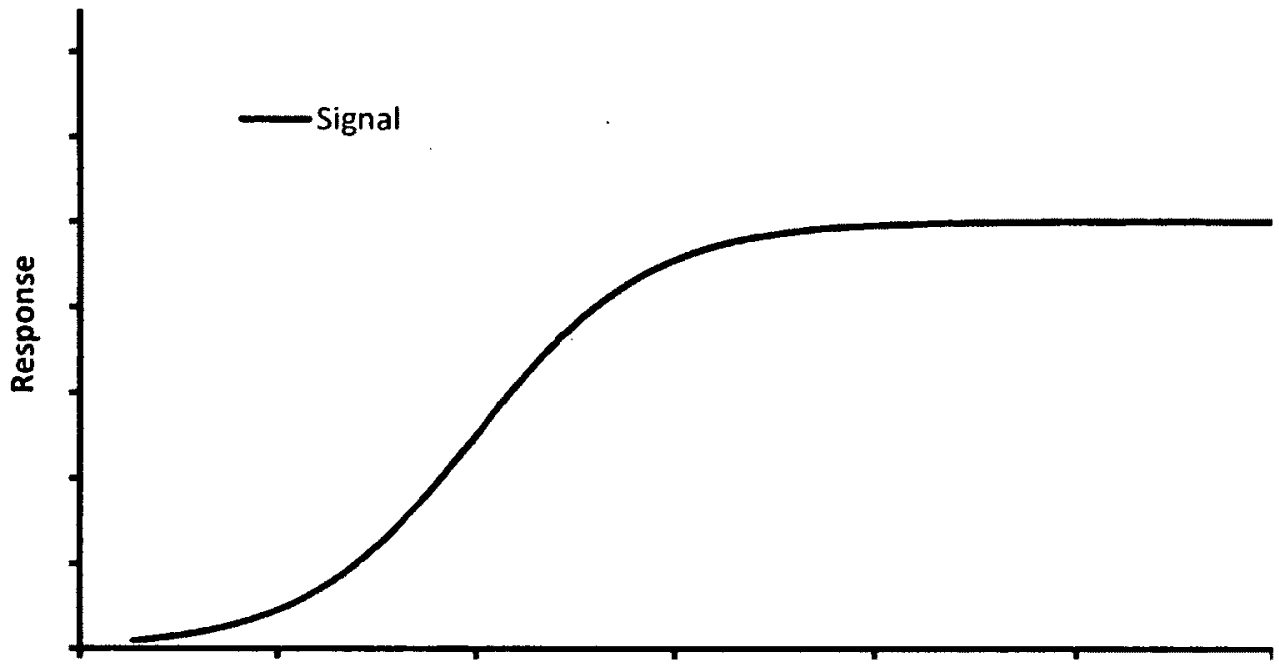

Increasing ligand concentration $\rightarrow$

B

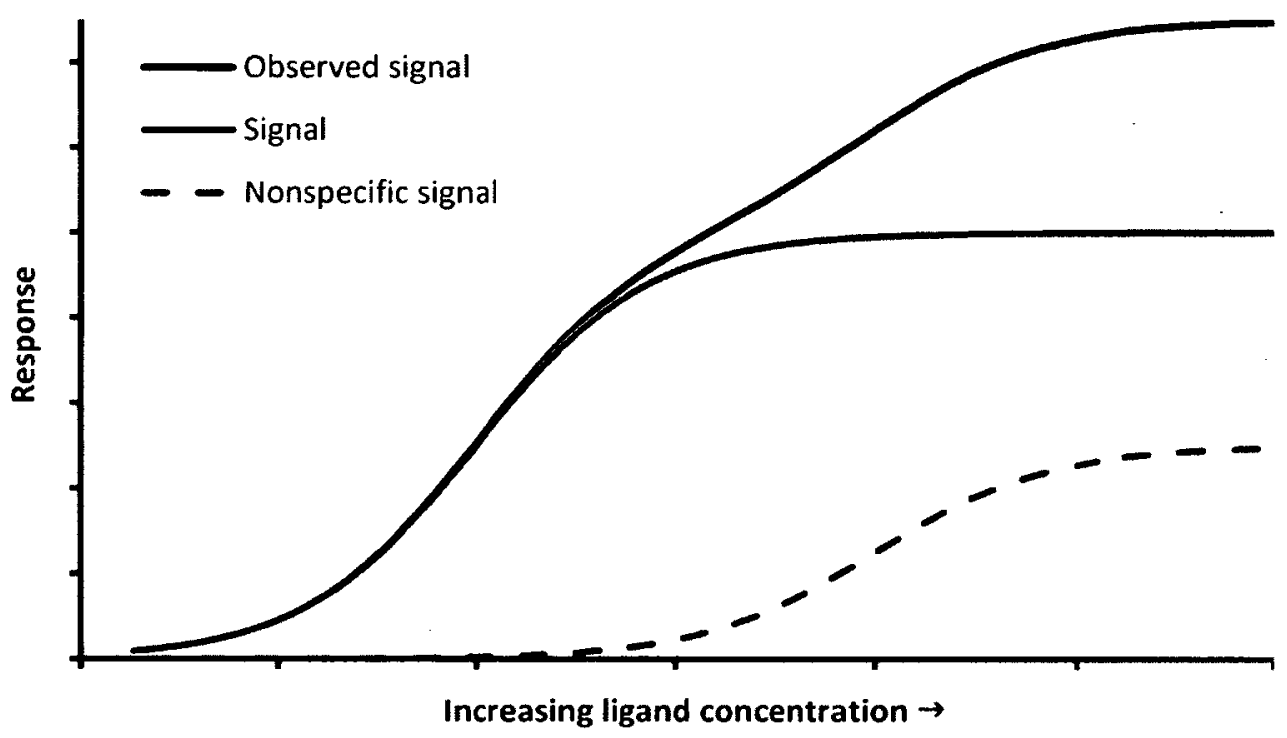

Figure 2.3. Typical binding isotherm for receptor ligand interactions. The observed response is a relative scale produced by the method of detection and the sensitivity of the experimental method. A) As the concentration of ligand is increased the receptor binding spots are saturated the signal reaches a distinct maximum. B) In the presence of a nonspecific interaction, specific signal can be masked by the combind signal but the combine signal will not conform to the rectangular hyperbola expected of a simple binding isotherm. 
As the behaviour of this isotherm is a measure of the fractional occupancy it can be mapped to many types of systems in which a signal is generated by the interaction of the aptamer and target. However, nonspecific interactions (Figure 2.3) can interfere with these signals which can be represented mathematically as linear (Equation 2.16) or additional binding isotherms (Equation 2.17; Motulsky and Christopoulos, 2004).

$$
\begin{gathered}
\text { Signal }=\operatorname{Signal1} \frac{[T]}{K_{d}+[T]}+\operatorname{Signal2}[T] \\
\text { Signal }=\operatorname{Signal1} \frac{[T]}{K_{d}+[T]}+\text { Signal2 } \frac{[T]}{K+[T]}
\end{gathered}
$$

While secondary binding does complicate the determination of binding affinities they can be avoided using appropriate experimental techniques. However, the primary difficulty associated with accessing the interactions of receptor and ligand is the fact that binding event themselves usually do not produce easily quantifiable changes. In the case of the dopamine DNA aptamer homolog this was particularly challenging and a number of different techniques were used to assess the binding affinity between the aptamer and the neurotransmitter. 


\section{Indirect measurements of Binding affinities}

One of the more common ways of getting around the difficulties associated with directly measuring binding between receptor and ligand is to allow establishment of the equilibrium and then partition the free ligand from the bound. This is usually accomplished by exposing the receptor to the ligand and then washing away free ligand. However, similar information can be obtained by sequestering the ligand to a matrix and exposing it to the receptor, which is more applicable to aptamer analysis as selection for aptamers usually rely on the sequestering of ligands of interest to a matrix for SELEX. Typically separation methods are usually solid support based but can also, in some cases, be accomplished with filtration or dialysis techniques. The major difficulty associated with using these techniques to determining binding affinities relates to shifts in the binding equilibrium which occurs during the separation of free ligand from bound. These separation techniques are also highly susceptible to the interference of nonspecific binding which can result from interactions with the solid support or filter (Figure 2.3; Jing and Bowser, 2011). Additionally, modification of either ligand or aptamer, by sequestering it to the matrix, adds additional uncertainty as to whether the measured affinity is representative of the true binding affinity between the aptamer and target. Due to these factors, column affinity studies are regarded as only useful for estimating binding constants (Phizicky and Fields 1995). Therefore, the work presented here initially used column affinity studies as an indicator of possible DNA homolog aptamer functionality. 


\section{Quartz crystal microbalance}

Another technique which uses fixed receptor or ligand to measure binding interactions is the quartz crystal microbalance. However, quartz crystal microbalance techniques have the advantage over column affinity related techniques in that no separation of bound and unbound ligand is required as the observed signal directly relates to the binding between ligand and receptor (Figure 2.4).

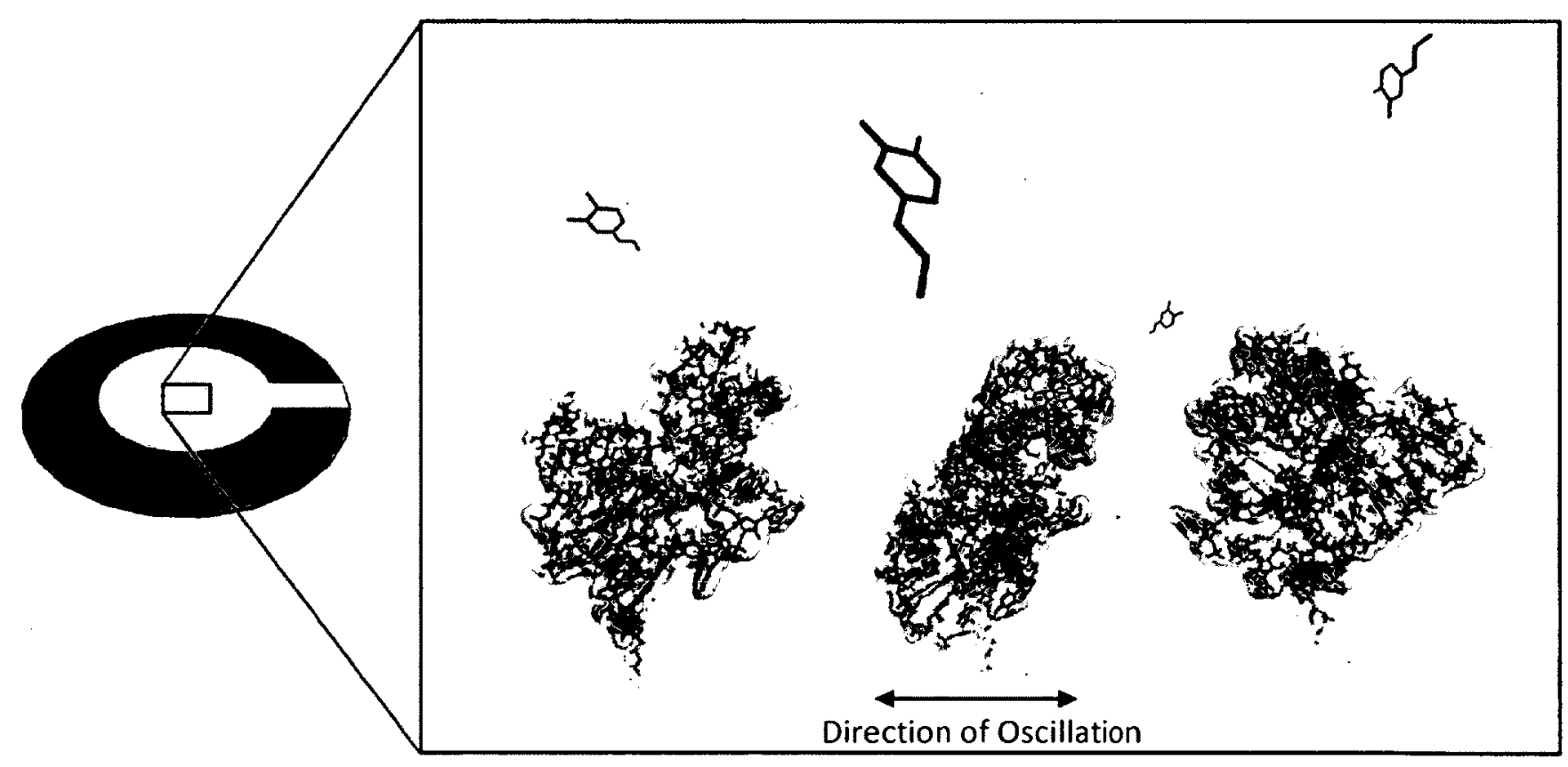

Figure 2.4. Schematic representation of the potential interaction of dopamine and the DNA homolog dopamine aptamer deposited on the gold electrode of the quartz crystal microbalance crystal. Change in frequency directly relate to the binding of the ligand to the receptor which increases resistance to the vibration of the resonator. 
Quartz crystal microbalances were developed based on the piezoelectric properties of quartz. The word piezoelectric derives from the Greek words piezein-elektro (pressure-electric) meaning that when mechanically-stressed quartz produces an electrical charge on the surface or conversely when exposed to electrical charge quartz will mechanically distort. When exposed to alternating current, quartz will oscillate in a direction perpendicular to the applied current (Cooper and Singleton, 2007). The frequency at which the quartz resonator oscillates is dependent on several factors as the resonator is actually a composition of the quartz crystal, the electrodes, any molecules deposited on the electrodes and the solution adjacent to the resonator. This relationship was initially described for surface coating relatirg the linear change in frequency $\left(\Delta f_{m}\right)$ to changes in mass on the surface of the crystal $\left(m_{s}\right)$ (Sauerbrey, 1959; Equation 2.18).

$$
\Delta f_{m}=-\frac{f_{o}^{2}}{F_{q} \rho_{q}} m_{s}
$$

In this equation the frequency of the native crystal resonance system $\left(f_{0}\right)$ is negatively affected by changes in the mass of the resonator based on frequency constant $\left(F_{q}\right)$ and density of the quartz crystal $\left(\rho_{q}\right)$. This relationship was expanded to include interactions with liquids that also contribute were observed to contribute to resonator properties (Kanazawa, 1997; Equation 2.19). 


$$
\Delta f_{l}=-\sqrt{\frac{f_{o}^{3} \eta_{l} \rho_{l}}{\pi \mu_{q} \rho_{q}}}
$$

This equation introduced the effect of surface/liquid shearing $\left(\mu_{q}\right)$ and when added to Sauerbrey's equation (Equation 2.18) formed the basis for theory relating to dampening of the resonator (Cooper and Singleton, 2007). This change in frequency associated with deposition or specific binding produced by functionalizing the surface has been used to directly measure binding constants for a variety of biological systems (Cooper and Singleton, 2007; Dixon, 2008; Becker and Cooper, 2011). However, there have been few examples of specific small molecule detection using QCM due to the nanogram to microgram per $\mathrm{cm}^{2}$ detection range associated with this technique (Dixon, 2008). In general, this technique has been most successful with molecules with larger molecular weights such as proteins and DNA (Cooper and Singleton, 2007; Dixon, 2008; Becker and Cooper, 2011) or molecules that have been functionalized with larger macromolecular structures such as nanoparticles to amplify the signal (Liu et al., 2004; Ha et al., 2004). However, there have been a few examples of the successful detection of small molecules such as the detection of dioxins (Kurosawa et al., 2006) and bisphenol A (Rahman et al., 2007) using antibodies. Carmen et al., (2004) were also able to describe a system in which the binding of glucose to its receptor produced a large signal ascribed to conformational changes induced by formation of the ligand receptor complex. In its free state, the Escherichia coli receptor forms a flexible dumbbell 
structure which closes on glucose when it binds. The more compact nature of the ligand receptor complex was attributed to an increase in surface rigidity producing the resistance induced signal. As aptamers are believed to undergo conformational changes upon target binding, the quartz crystal microbalance was used to examine the binding affinity between the DNA homologue of the RNA dopamine aptamer and dopamine. Similar studies were used to examine the potential binding of the DNA homolog of the RNA B12 aptamer.

Additional methods which were utilized in these studies used more direct ways of investigating the binding of the DNA homologs. These methods, isothermal titration calorimetry, electophoretic gel shifts and fluorescence anisotropy have the advantage of not requiring the ligand or receptor to be attached to a solid support. This allows for a higher degree of confidence that attachment of the ligand or receptor to a matrix has not affected the properties of the molecule skewing the measurable affinity.

\section{Isothermal titration calorimetry}

The development of isothermal titration calorimetry within the past couple of decades has allowed for the direct measurement of the small heat of reaction associated with biological and aptamer binding interactions (Lin et al., 2008; Jing and Bowser 2011). This ability to measure the heat of reaction (q) for binding events, has allowed for the determination of the binding enthalpy $(\Delta \mathrm{H})$, binding entropy $(\Delta S)$ and 
direct measurement of binding constants (Salim and Feig, 2009). Modern isothermal titration calorimetry instruments monitor changes in heat by recording the change in heat of the reaction relative to a reference cell composed of a similar solution (Figure 2.5). Generally, the ligand is titrated into a solution of the receptor and mixed to speed up equilibration. Using this set up it is possible to observe generated or absorbed heat produced by the molecular binding, but heat may also result from conformational changes, solvent effects, dilution effects and additionally the stirring process. Therefore, appropriate controls need to be run to ensure observed heat elution or absorption is attributed properly (Salim and Feig, 2009).

The amount of heat produced by the binding of the receptor ligand system $\left(q_{i}\right)$ can be described as a function of the fractional association $(F)$ between the two molecules (Equation 2.20). Where the total heat is related to the stoichiometry $(n)$, the change in enthalpy produced by the binding $(\Delta H)$, the assay volume $(V)$ and the total receptor concentration ([Ao]; Salim and Feig, 2009).

$$
q_{i}=n F\left[A_{o}\right] \Delta H V
$$

The fraction bound can be determined from a polynomial equation similar to equation 2.10 (Equation 2.21) which relates the binding between aptamer and target to the binding constant ( $K_{B} ;$ Salim and Feig, 2009). 


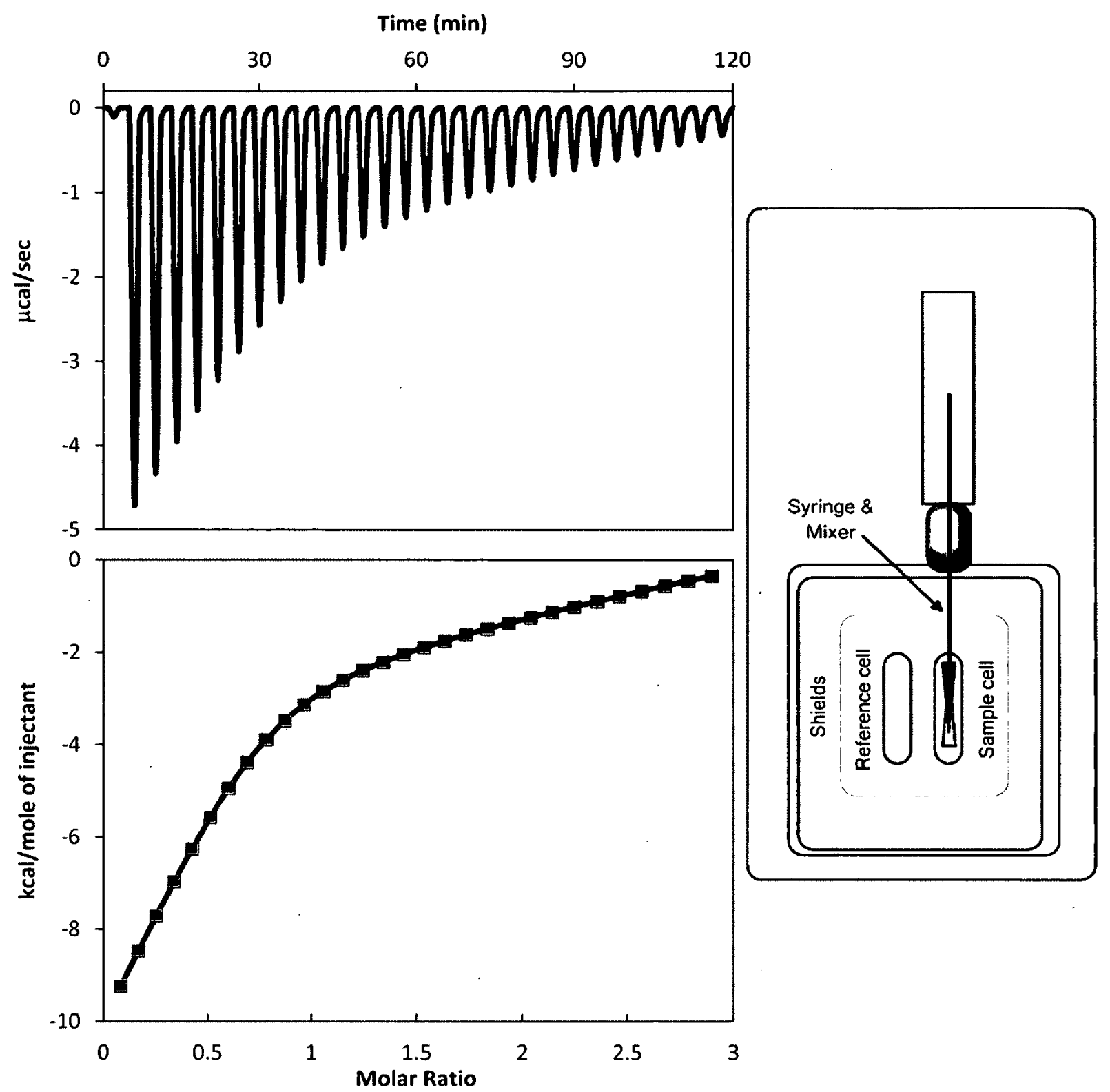

Figure 2.5. Schematic representation of the heat evolved from a binding reaction and the experimental setup of a isothermal titration apparatus where a sample and reference cell are kept at constant temperature by feedback heater systems that measure the change in heat by the energy required to re-establish equilibrium. 


$$
F^{2}-\left(1+\frac{\left[T_{o}\right]}{n\left[A_{o}\right]}+\frac{1}{n K_{B}\left[A_{o}\right]}\right) F+\left(\frac{\left[T_{o}\right]}{n\left[A_{o}\right]}\right)=0
$$

Therefore, solving for the fraction bound using the quadratic equation allows for determination of the binding affinity between aptamer and target using a nonlinear fitting approach (Salim and Feig, 2009).

Isothermal titration calorimetry was accessed for its utility in determining the binding affinity between the DNA homolog of the dopamine aptamer and dopamine in these studies.

\section{Electrophoretic gel shift assays}

Electrophoresis is one of the fundamental methods for the separation of biological molecules. The technique relies principally on the forced migration of biological molecules through a matrix or gel driven by. an electric field. The migration rate of individual molecule relates to its size, conformation and charge. The size and conformation providing a migrational resistance relative to the matrix pore size while the charge contributes to the mobility of the molecule in the electric field (Wilson and Walker, 2005). This has allowed for the discrete separation of molecules into bands based on their migration rate through the gel. These migrational patterns have been used to derive information about the properties of the molecules being analysed as well as for the purification and isolation of molecules (Wilson and Walker, 2005). 
In the electrophoretic mobility of nucleotides, the negatively charged phosphate backbone is the most significant contributor as it provides a very similar mass to charge ratio for molecules which is independent of length. The effect of this charge ratio is particularly evident in the migrational patterns observed with double stranded DNA which due to base pairing maintains its long linear structure resulting in a separation based almost exclusively on length (Wilson and Walker, 2005). The migration of single stranded nucleotides however is not dependent on length due to the tendency of the single stranded molecules to form intramolecular base pairing. The tertiary structures, resulting from this base pairing, produce variable resistance to gel migration based on matrix pore size. Single stranded nucleotides can be separated based on length using denaturing conditions which disrupts the intramolecular hydrogen binding between base pairs. To achieve this single stranded nucleotide samples are usually prepared using a combination of heat and either urea or formamide. Heating disrupts the base pairing allowing interactions with formamide or urea to replace the intramolecular binding (Wilson and Walker, 2005).

The migration of nucleotides through a matrix is dependent on the applied electrical force on the charged molecules and the resistance of the matrix to the progress of their migration. The velocity of their mobility is defined using the following relationship (Equation 2.22). 


$$
v=\frac{E q}{f}
$$

In this equation, the rate $(v)$ is directly proportional to the potential gradient $(E)$ and the charge of the molecule $(q)$. The rate is also inversely proportional to the frictional coefficient $(f)$ (Wilson and Walker, 2005).

While electrophoretic methods based on the migration rate of double stranded DNA or linearized single stranded nucleotides have found use in techniques such as sequencing and isolation, non-denaturing conditions have been used to characterize sequence based protein binding profiles, binding constants and conformational sequence polymorphisms. These non-denaturing techniques have been shown to be directly applicable to the determination of aptamer and protein binding constants (Tahiri-Alaoui et al., 2002). Non-denaturing methodologies have also been used in sequence mutation analysis using double stranded DNA. This technique known as heteroduplex analysis demonstrates the sensitivity of electrophoretic analysis as a single nucleotide mismatch is enough to produce a kink in the double stranded helix decreasing the migration rate relative to the helix not containing the mismatch (Børresen AL. 2002). This high degree of sensitivity to slight conformational changes induced in the tertiary structure of the DNA makes electrophoretic techniques ideal for detecting conformational induced changes in the structure of aptamers produced by 
ligand binding, as the mobility of the complex would change relative to the free aptamer.

In this study the electrophoretic mobility of the DNA homolog of the dopamine aptamers was examined as a potential method for accessing the binding affinity between the aptamer and its target.

\section{Anisotropy studies}

Fluorescence anisotropy is another technique which lends itself well to binding studies by providing a method of directly relating changes in fluorescent signal with binding events. Fluorescence anisotropy utilizes the polarization of light to selectively excite a portion of a fluorophore population based on their relative orientation to the light source (Jing and Bowser 2011).

Light can be rationalized as the propagation of an electromagnetic pulse characterized by perpendicular oscillations of the electric and magnetic fields relative to each other. The linear propagation of light from a radiation source produces light oscillating in an unoriented manner (Jameson and Ross, 2010; Figure 2.6). 


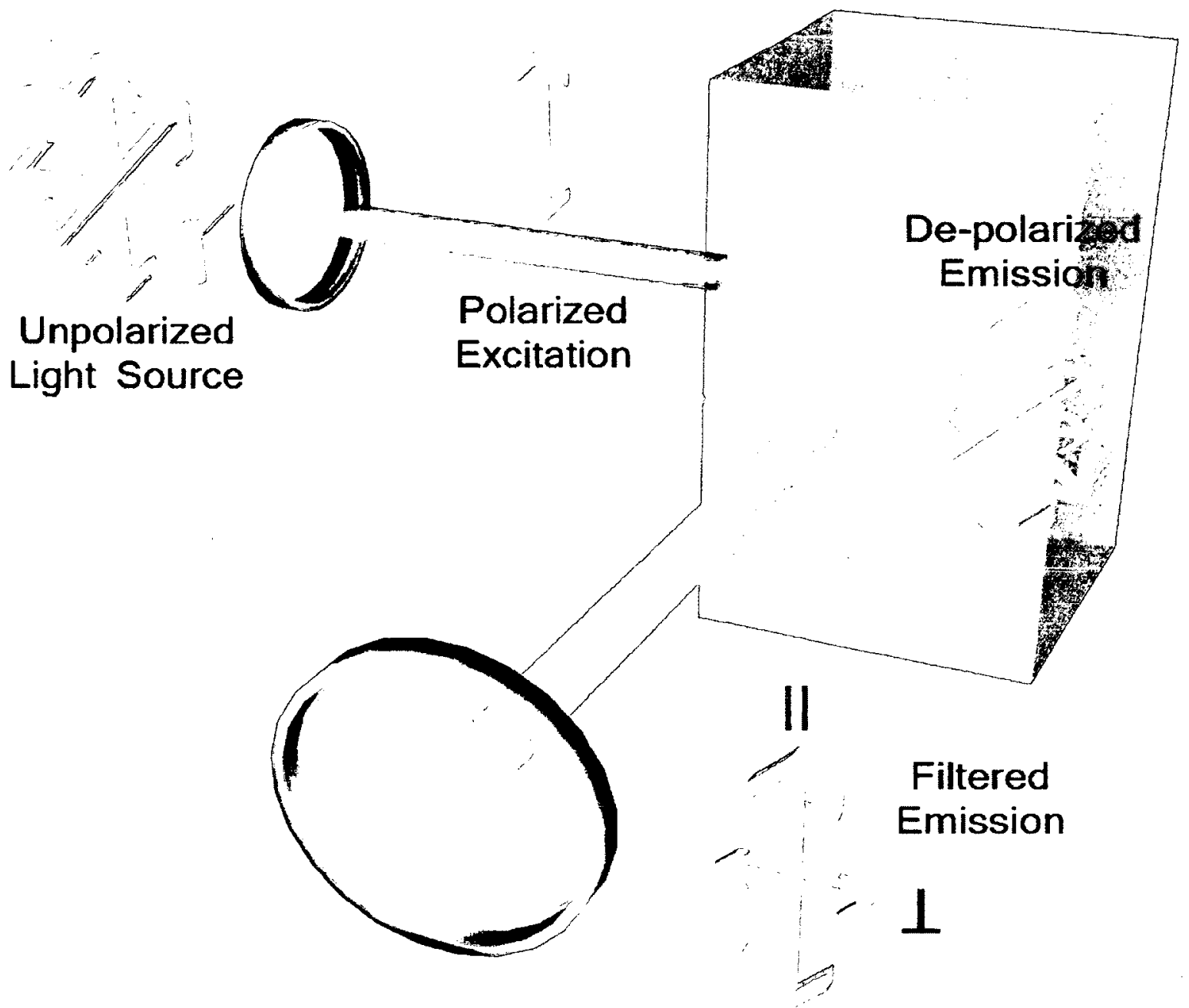

Figure 2.6. Schematic of the instrumentation involved in fluorescence anisotropy studies. Typically light is filtered to produce vertically oriented light for photoselective fluorophore excitation. Both the vertical $\left(I_{\|}\right)$and horizontal $\left(I_{\perp}\right)$ fluorophore emission intensities are collected at $90^{\circ}$ to the incident of the excitatory light source. 
In anisotropic studies, a polar light filter is used to deflect light which is not oriented in the proper direction producing a polarized light beam that is used to excite the fluorophore sample. Due to the polarized nature of the light, it is only able to excite fluorophores with their dipoles oriented parallel to the propagation of the light (Mann and Krull, 2003; Jameson and Ross, 2010). This results in only a select portion of the fluorophore population being excited by the incident light which quickly falls out of alignment due to Brownian rotation. The characteristic exponential decay associated with fluorescent excitation in congruency with the Brownian rotation of the excited fluorophores results in a distinct pattern that provides information relating to the molecules motion in the solution (Jameson and Ross, 2010; Figure 2.7). Of particular interest in anisotropic studies is the rotation of the excited fluorophore along its axes in three-dimensional space.

Similarly to regular fluorescent studies, the fluorescent emissions of the fluorophore are recorded at $90^{\circ}$ to the excitation. However, these readings are performed through an optical filter which is rotated to record emissions intensity polarized parallel $\left(I_{\|}\right)$to the orientation of the orientation polarized excitatory light, as well as the intensity of emission perpendicular $\left(I_{\perp}\right)$ to the orientation of the excitatory light source. The intensity of the emission signal relates to the excitation lifetime of the fluorophore and the rotational freedom of the molecule. 


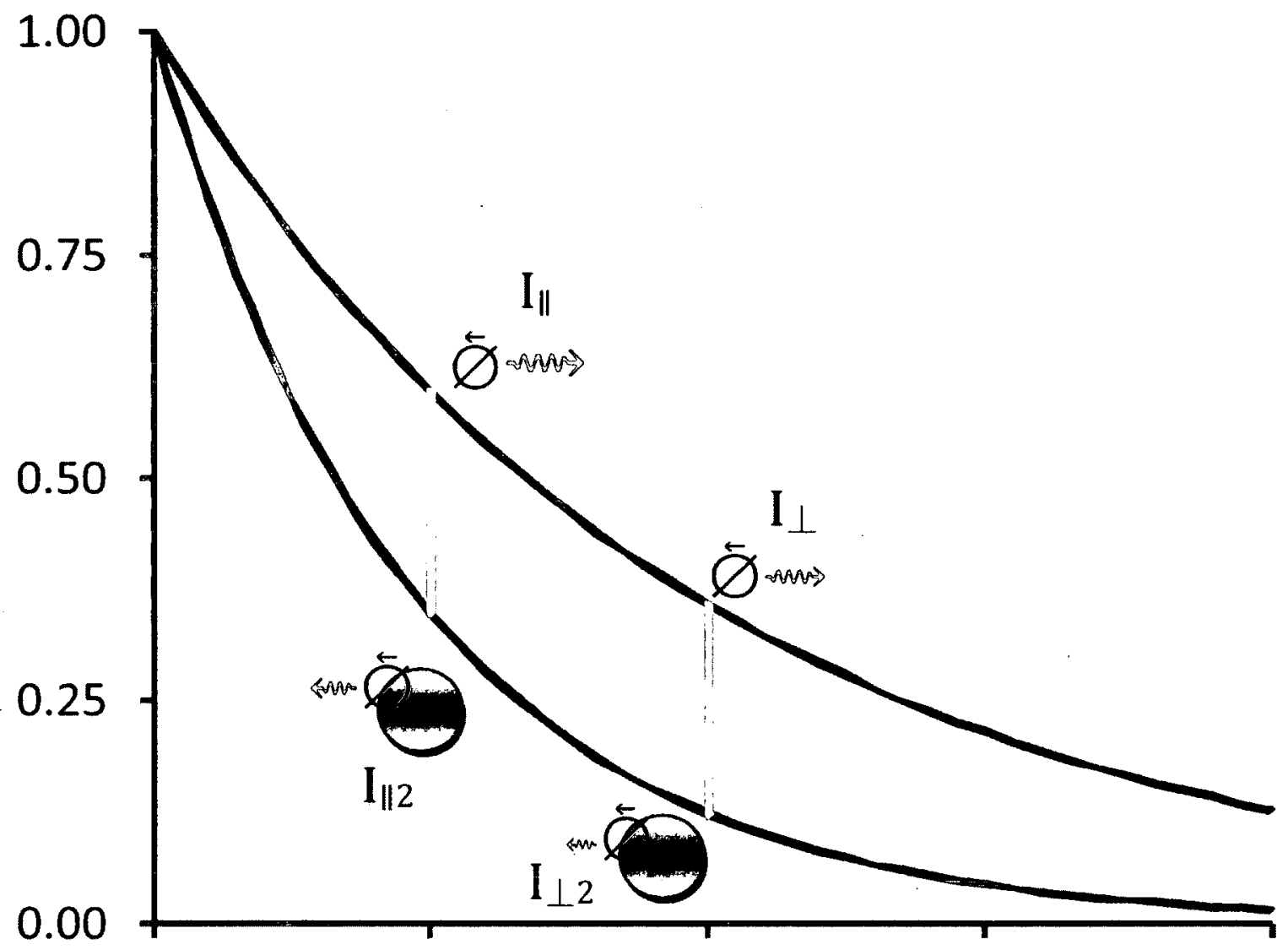

Figure 2.7. Schematic representation of the drop in signal emission intensity ( $m \rightarrow$ ) resulting from the reduction of fluorophore $(\varnothing)$ rotational freedom. This reduction of rotational freedom is produced by the change in mass associated with the formation of a receptor ligand complex. The measurement of emission intensity in the parallel $\left(I_{\|}\right)$ and perpendicular $\left(I_{\perp}\right)$ orientations allows the collection of signal at increasing rotational distance from the excitation orientation. Introduction of molecules that binds to the fluorophore decreases the fluorophores rotational speed causing a reduction in the number of fluorescent molecules that will be able to reach the parallel $\left(I_{\| 2}\right)$ and perpendicular $\left(I_{\perp 2}\right)$ orientations before emitting. 
By measuring the emission intensity at two different orientations $\left(I_{\|}, I_{\perp}\right)$ the emission intensity of the fluorophore is assessed at increasing rotational distance from the excitation orientation providing information relating to the fluorophores environment. This is particularly useful for determining binding events as the relative rotational speed of molecules in solution relates to their mass, smaller rotating faster relative to larger molecules. Therefore, a fluorophore that has its mass increased will produce less emission intensity $\left(I_{\|}, I_{\perp}\right)$, relative to the free fluorophore as its rotational velocity is reduced while its lifetime is unaltered (Figure 2.7). This means that the emission intensity of a population of fluorophores shifts from the maximal free signal to a minimum bound emission signal which is directly related to the fraction of the population bound (Figure 2.7). The decrease in the emission is usually expressed as changes in polarization or anisotropy where both types of measurement are ratios of the parallel and perpendicular emission values. The polarity is calculated as the difference between the signals over the total signal (Equation 2.23) and the anisotropy is the difference divided by the parallel emission and two times the perpendicular emission (Equation 2.24; Jing and Bowser 2011).

$$
P=\frac{I_{\|}-I_{\perp}}{I_{\|}+I_{\perp}}
$$




$$
A=\frac{I_{\|}-I_{\perp}}{I_{\|}+2 I_{\perp}}
$$

Both types of data relate directly to the amount of bound versus free fluorophore and can be used indirectly to determine dissociation constants in solution.

Fluorescence anisotropy was used to examine the binding of both DNA homologs with their respective ligands in this study and proved to be the most effective technique for determining the binding affinity resulting from the conversion of these aptamers to their DNA homologs.

\section{Materials and Methods}

1-O-Dimethoxytrityl-hexyl-disulfide, 1'-[(2-cyanoethyl)-(N,N-diisopropyl)]-

phosphoramidite (Thiol-Modifier C6 S-S), 6-(3',6'-dipivaloylfluoresceinyl-6carboxamido)-hexyl-1-O-(2-cyanoethyl)-(N,N-diisopropyl)-phosphoramidite Fluorescein Phosphoramidite) and the phosphoramidites of A, T, C and G for DNA synthesis were acquired from Glen Research.

The Quant-iT OliGreen ssDNA Assay Kit was acquired from Invitrogen

\section{DNA synthesis}

DNA was synthesized on a Mermade 6, Bioautomation using standard phosphoramidite chemistry. 


\section{DNA Sequences}

DNA homologue of the RNA dopamine aptamer (Mannironi et al., 1997) was produced to characterize its ability to bind dopamine. The $5^{\prime} \mathrm{N}$ denotes the position of the various modifications used to functionalize the aptamer. In the case of fluorescent studies the $\mathbf{N}$ denotes fluorescein, in the case of $\mathrm{qcm}$ studies this was replaced with a thiol-group. The $\mathrm{N}$ position was omitted when the unmodified sequence was produced.

\section{DNA1}

\section{5'-NGTCTCTGTGTGCGCCAGAGACACTGGGGCAGATATGGGCCAGCACAGAATGAGGCCC-3'}

Additionally, several other sequences were produced to characterize the DNA form of the dopamine aptamer. The sequences were denoted DNA2-5 to distinguish them from the original DNA homolog of the RNA aptamer (DNA1 above).

\section{DNA2}

5'-GTCTCTGTGTGCAACAGAGACACTGGGGCAGATATGGGCCAGCACAGAATGAGGCCC-3'

DNA3

5'-GTCTCTGTGTGCGCCAGAGACACTGGGGCAGATATGGGCCCGCACAGAATGAGGCCC-3'

DNA4

5'-GTCTCTGTGTGCGCCAGAGACACTGGGGCAGATATGGGCCAGCACAGAATCCGGCCC-3' 
DNA5

5'-GTCTCTGTGCCAGCCAGAGACACTGGGGCAGATATGGGCCAGCACAGAATGAGGCCC-3'

The DNA homolog of the RNA B12 aptamer (Lorsch and Szostak, 1994) was also produced to characterize its ability to bind $\mathrm{B} 12$. The $5^{\prime} \mathrm{N}$ denotes the position of the various modifications used to functionalize the aptamer. In the case of fluorescent studies the $\mathrm{N}$ denotes fluorescein, in the case of qcm studies this was replaced with a thiol-group.

5'-NGGAACCGGTGCGCATAACCACCTCAGTGCGAGCAA-3'

\section{Column studies using Oligreen}

The Quant-iT OliGreen ssDNA Assay Kit was used to analyse the amount of DNA retained by the dopamine column as it was exposed to increasing concentrations of the aptamer. A $0.1 \mathrm{~g}$ dopamine column was pre-equilibrated with a buffer consisting of 50 $\mathrm{mM}$ Tris $\mathrm{HCl}(\mathrm{pH} 7.4), 5 \mathrm{mM} \mathrm{MgCl} 2,0.5 \mathrm{M} \mathrm{NaCl}$ and $0.02 \%$ ascorbic acid in a $0.4 \mu \mathrm{m}$ filter centrifuge tube. The column was then incubated with increasing concentrations of the DNA aptamer before being centrifuged for 20 minutes at $10,000 \mathrm{~g}$. The column was then washed with $0.5 \mathrm{mLs}$ of hot buffer $\left(\sim 85^{\circ} \mathrm{C}\right)$. Samples were prepared for DNA content analysis by mixing half and half with Quant-iT Oligreen ssDNA reagent diluted 200 fold in $10 \mathrm{mM}$ Tris- $\mathrm{HCl}, 1 \mathrm{mM}$ EDTA, $\mathrm{pH}$ 7.5. Samples were analyzed on a Varian Cary Eclipse Fluorescence Spectrophotometer $(E x / E m=498 / 518 \mathrm{~nm})$. 


\section{Column studies using fluorescein labelled aptamer}

A $0.1 \mathrm{~g}$ dopamine column was pre-equilibrated with a buffer consisting of $50 \mathrm{mM}$ Tris $\mathrm{HCl}(\mathrm{pH} 7.4), 5 \mathrm{mM} \mathrm{MgCl}, 0.5 \mathrm{M} \mathrm{NaCl}$ and $0.02 \%$ ascorbic acid in a $0.4 \mu \mathrm{m}$ filter centrifuge tube. The column was then incubated with increasing concentrations of the DNA aptamer before being centrifuged for 20 minutes at 10,000g. The column was then washed with $0.5 \mathrm{mLs}$ of hot buffer $\left(\sim 85^{\circ} \mathrm{C}\right)$. Samples were analyzed for DNA content on a Varian Cary Eclipse Fluorescence Spectrophotometer (Ex/Em $=498 / 519 \mathrm{~nm})$.

\section{Quartz crystal microbalance}

Quartz crystal microbalance (QCM) studies were performed using a QCM25 Crystal Oscillator and QCM100 Analog Controller and flow cell all of which were acquired from Stanford Research Systems, Inc.

QCM crystals ( $2.54 \mathrm{~cm}$ Dia. $331 \mu \mathrm{m}$ thickness, $5 \mathrm{MHz}$ crystal) were initially cleaned through a brief emersion in aqua regia solution consisting of one part concentrated nitric acid and 3 parts concentrated $\mathrm{HCl}$. The crystal was then thoroughly washed with distilled water and mounted in the flow cell $(\sim 0.15 \mathrm{~mL}$ volume). The flow cell was preequilibrated with buffer consisting of $50 \mathrm{mM}$ Tris $\mathrm{HCl}(\mathrm{pH} 7.4), 5 \mathrm{mM} \mathrm{MgCl}, 0.5 \mathrm{M} \mathrm{NaCl}$ and $0.02 \%$ ascorbic acid followed by introduction of the thiolated aptamer suspended in the same buffer. $0.1 \mathrm{M} 6-$ Mercaptohexanol in buffer was then introduced to the flow cell to produce a hydroxyl monolayer. The ability of the aptamer to bind dopamine on 
the modified surface was assessed by exposing the surface to increasing concentrations of dopamine. All solutions were introduced into the flow cell in a batch sample loading method using a gravity-feed siphon to reduce potential interference resulting from shearing forces.

Quartz crystal microbalance studies with the DNA homologue of the RNA B12 aptamer were performed by preequilibrating a cleaned QCM crystal with $1 \mathrm{M} \mathrm{LiCl,} 5 \mathrm{mM}$ $\mathrm{MgCl}_{2}$, and $25 \mathrm{mM} \mathrm{HEPES}(\mathrm{pH} 7.4)$ in the flow cell. Following equilibration the thiolated aptamer suspended in the HEPES buffer was introduced and the surface was backfilled with $0.1 \mathrm{M}$ 6-Mercaptohexanol in HEPES buffer. Potential binding between the aptamer and B12 on the modified surface was assessed by exposing the surface to increasing concentrations of B12.

\section{Isothermal titration calorimetry}

Isothermal titration calorimetry experiments where performed using a MicroCal $\mathrm{iTC}_{200}$ isothermal titration calorimetry (ITC) instrument. Dopamine $(0.47 \mathrm{mM})$ and $20 \mu \mathrm{M}$ samples of the DNA version of the RNA Dopamine aptamer were suspended in the working buffer of $0.02 \%$ ascorbic acid, $5 \mathrm{mM} \mathrm{MgCl}_{2}, 0.5 \mathrm{M} \mathrm{NaCl}$ and $50 \mathrm{mM}$ Tris $\mathrm{HCl} \mathrm{pH}$ 7.4. All data, was primarily, generated by sequential injections of dopamine into the 1.4 $\mathrm{mL}$ sample cell. Potential binding between the aptamer and dopamine was assessed by varying the injection volume of the dopamine, the effective range of the molar ratio between dopamine and the aptamer and the constituents of the buffer. 


\section{Electrophoretic gel shift assay}

Gel shift assays were performed using a modification of the standard gel electrophoresis method in that gels were cast in the absence of urea to maintain nondenaturing conditions and also with varying percentages of acrylamide to determine the optimum conditions for separating the free aptamer from the aptamer dopamine complex. Gradient gels were prepared using a gradient mixer constructed according to the methodology described by Shearer (1994).

Samples were run on gels cast with $18 \times 16 \mathrm{~cm}$ gel plates. Twelve percent acrylamide gels were cast by combining varying volumes of $5.3 \mathrm{M}$ acrylamide and 130 $\mathrm{mM} \mathrm{N}, \mathrm{N}^{\prime}$-methylenebisacrylamide solution, $15 \mathrm{mLs}$ of $0.44 \mathrm{M}$ Boric acid, $0.44 \mathrm{M}$ Tris, and $10 \mathrm{mM}$ EDTA. Distilled $\mathrm{H}_{2} \mathrm{O}$ was used to bring the total volume to $52.5 \mathrm{mLs}$. The mixture was heated to $37^{\circ} \mathrm{C}$ filtered and degassed. Once the sample had reached room temperature $0.45 \mathrm{mLs}$ of $10 \%$ ammonium persulfate was mixed in followed by the addition of $35 \mu \mathrm{L}, N, N^{\prime}, N^{\prime}$-tetramethylethylenediamine. A gradient mixer was used to produce gradient gels while regular gels were simply poured and allowed to polymerize for 20 minutes. After polymerization the gels were set up in a Hoefer SE 600 series vertical slab gel electrophoresis unit and the buffer reservoirs were filled with $88 \mathrm{mM}$ Boric acid, $88 \mathrm{mM}$ Tris, and $2 \mathrm{mM}$ EDTA. Gels were typically preequilibrated using a constant current of 35 milliamps for 20minutes using a FisherBiotech Power Suppy FB1000. 
Non-denatured aptamer samples were prepared for gel electrophoresis by combining $10 \mu \mathrm{L}$ of buffer $10 \%(v / v)$ glycerol with $10 \mu \mathrm{L}$ of the aptamer $8 \mu \mathrm{M}$ suspended in buffer. Non-denatured samples of the aptamer dopamine complex were prepared for gel electrophoresis by combining $10 \mu \mathrm{L}$ of $200 \mathrm{mM}$ Dopamine suspended in buffer $10 \%$ $(v / v)$ glycerol with $10 \mu \mathrm{L}$ of the aptamer $8 \mu \mathrm{M}$ suspended in buffer.

\section{Anisotropy studies with fluorescein labelled aptamer}

Anisotropy studies of fluorescein labelled aptamer were used to assess decreases in rotational freedom in the presence of increasing concentrations of dopamine. All samples were prepared in $50 \mathrm{mM}$ Tris $\mathrm{HCl}(\mathrm{pH} 7.4), 5 \mathrm{mM} \mathrm{MgCl}, 0.5 \mathrm{M} \mathrm{NaCl}$ with the aptamer concentration being held constant as dopamine concentrations were varied. Fluorescent data was collected with a $40 \mu \mathrm{L}$ fluorescent cell with a FluoroLog-3 Spectrofluorometer from HORIBA Jobin Yvon (Ex/Em = 498/519 nm).

Anisotropy studies with the DNA homologue of the fluorescein labelled RNA B12 aptamer were performed by incubating the aptamer at constant concentration in increasing concentrations $\mathrm{B} 12$. All samples were prepared in $1 \mathrm{M} \mathrm{LiCl}, 5 \mathrm{mM} \mathrm{MgCl}$, and $25 \mathrm{mM}$ HEPES (pH7.4). Fluorescent data was collected with a $40 \mu \mathrm{L}$ fluorescent cell with a FluoroLog-3 Spectrofluorometer from HORIBA Jobin Yvon (Ex/Em $=498 / 519 \mathrm{~nm}$ ). 


\section{Anisotropy studies using target as the reporter molecule}

Anisotropy studies using targets as the reporter molecule (dopamine, norepinephrine and tyramine) were performed by exposing the targets to increasing concentrations of the unlabelled aptamer. All samples were prepared in $50 \mathrm{mM}$ Tris $\mathrm{HCl}$ (pH 7.4), $5 \mathrm{mM} \mathrm{MgCl}, 0.5 \mathrm{M} \mathrm{NaCl}$ with the concentration of dopamine being held constant as the aptamers concentrations was increased. Fluorescent data was collected with a $40 \mu \mathrm{L}$ fluorescent cell with a FluoroLog-3 Spectrofluorometer from HORIBA Jobin Yvon (Ex/Em = 285/317 nm; Hoss and Smiley 1977).

Additional anisotropy studies of catechol-aptamer interactions with the dopamine aptamer were performed by exciting the samples at $285 \mathrm{~nm}$ and observing the emission at $317 \mathrm{~nm}$.

\section{Data fitting for dissociation constant determination}

Anisotropy values used to determine binding affinities were taken as the median of six values. Dissociation constants and binding curve fits were determined by fitting the observed data to binding equations using the solver feature of Microsoft Excel (Fylstra et al., 1998; Nenov and Fylstra 2003; Kemmer and Keller 2010). 


\section{Results}

Initial indications of the ability of the DNA homologs of the RNA dopamine and RNA B12 aptamers to bind to their respective ligands were suggested by affinity chromatography. Therefore, initially the dissociation constant for the DNA dopamine aptamer was assessed using a dopamine column and the unlabeled DNA sequence. Passing increasing concentrations of the dopamine aptamer through the columns resulted in increasing amounts of the aptamer being retained by the column as determined by the use of the DNA Oligreen detection kit (Figure2.8). These studies suggested that if the observed binding had any specificity towards the dopamine column it was buried in potential nonspecific interaction with either the column or filter used in the assays. This is quite apparent as binding saturation was not achieved as is indicated by the lack in signal plateau associated with the regular binding isotherm. However, fitting of binding isotherms to the data taking into account the nonspecific binding suggested that the specific binding affinity between aptamer and the column was found to be around $7 \mu \mathrm{M}$.

These experiments used too broad of a concentration range as the Log 10 dilution series was initially inspected to find the concentration range in which the aptamer interacted with the column. In order to reduce interference in the model fitting, associated with the nonspecific interaction with the column, the experiment was repeated three times with a narrower aptamer concentration range (10-1000 nM). These studies supported the initial binding constant even suggesting it was somewhat better $(2.34 \mu \mathrm{M})$ than the initial study but the data was also fairly noisy. 


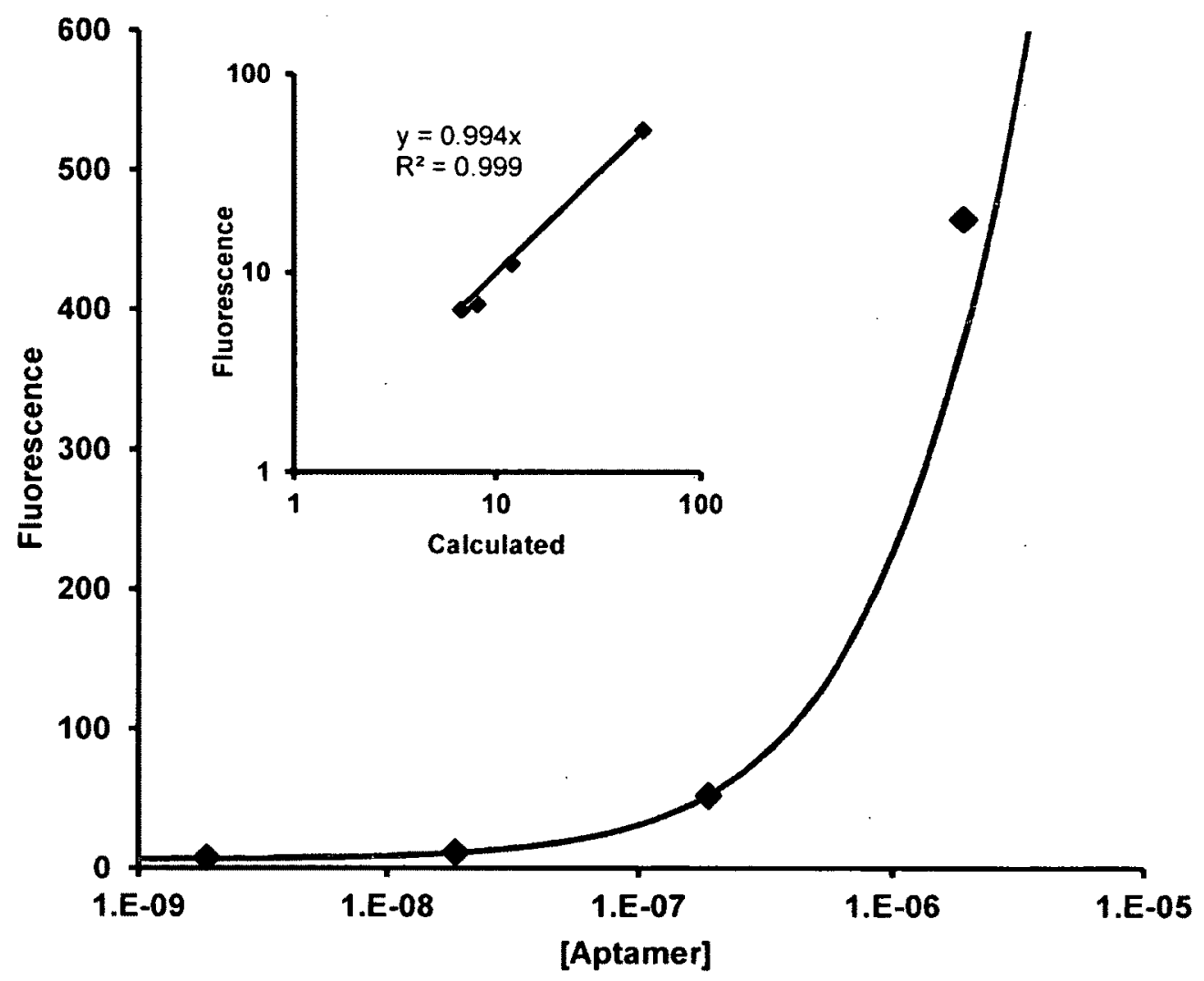

Figure 2.8. Analysis of the binding of the DNA homolog of the RNA aptamer to the dopamine column using Oligreen. Oligreen was used to quantify the amount DNA retained on the dopamine column as increasing concentrations of the aptamer were exposed to the dopamine column. The gray line represents the fit of the data to the binding isotherm and the insert represents the linearity between the observed and calculated values. 
The extra step associated with using Oligreen to fluorescently detect the amount of aptamer that was retained by the column was suggested as a potential source of error in the determination of the binding affinity between the aptamer and the column. Therefore, the fluorescein labelled aptamer was utilized, reducing the steps involved in sample preparation and allowing for direct fluorescent analysis of the DNA retained by the column (Figure 2.9). The results of these studies demonstrated that the fluoresceinlabelled aptamer also was affected by nonspecific interactions with the column although to a lesser degree than observed with the experiments using Oligreen as the DNA reporter. This change may have resulted from the addition of the fluorescein label to the sequence as the alteration of the sequence may have resulted in an alteration of its binding specificity. However, the collected data suggested that the fluorescein labelled aptamer had an affinity for dopamine around $16 \mu \mathrm{M}(\mathrm{N}=3)$. This represented a value approximately 10 fold that of the dissociation constant reported by Mannironi et al., (1997) for the RNA dopamine aptamer. However, while noisy, the range of values associated with the column binding studies suggested that the DNA form of the RNA dopamine aptamer had a dissociation constant in a similar range to that reported with the original RNA aptamer.

The difficulties with generating reliable binding constants with these studies can be mainly attributed to nonspecific interactions between the aptamer and the columns (Figure 2.9; Jing and Bowser, 2011). 


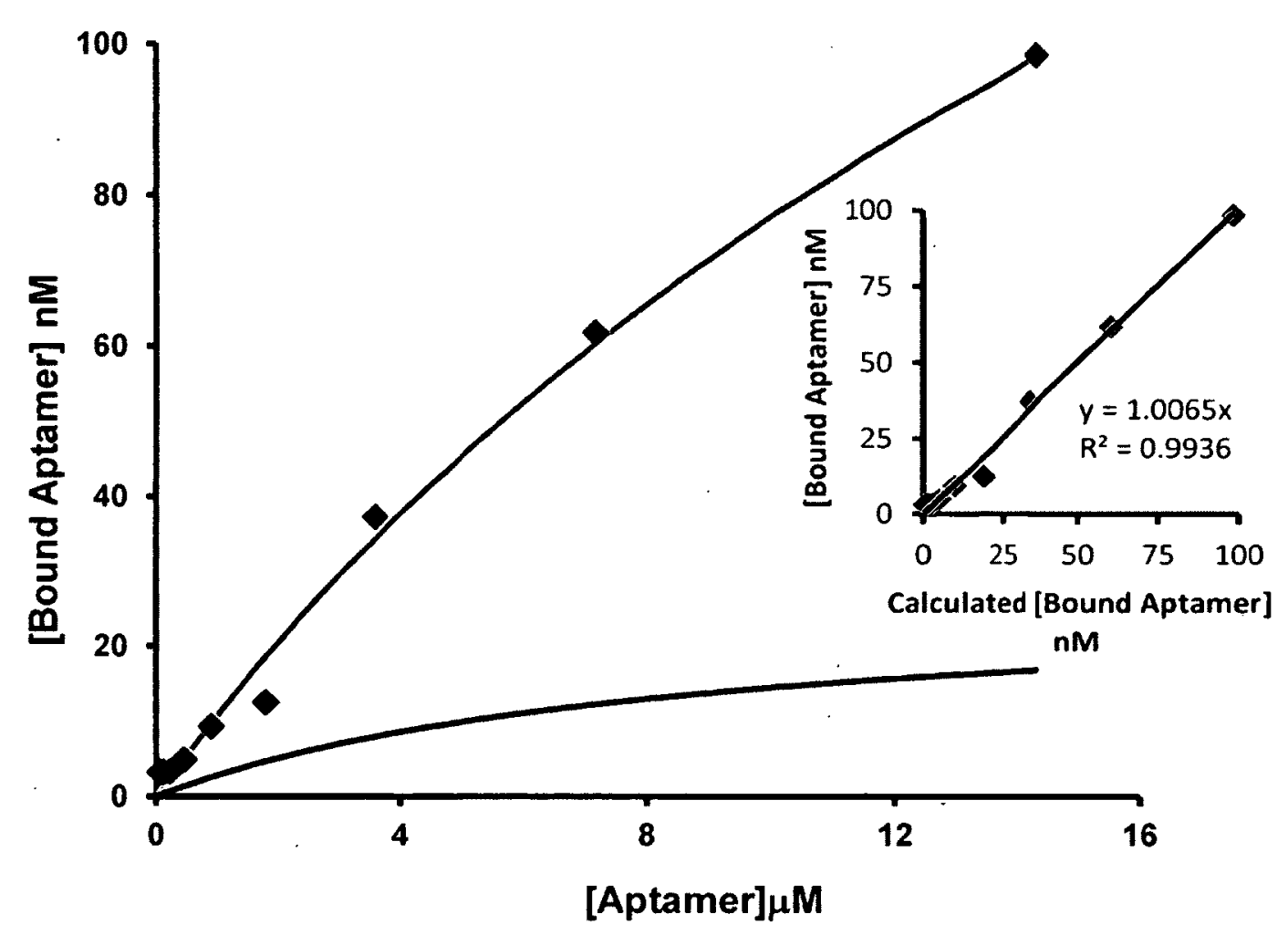

Figure 2.9. Analysis of the binding of the fluorescein labelled DNA homolog of the RNA aptamer to the dopamine column. The fluorescein tag was used to quantify the amount DNA retained on the dopamine column as increasing concentrations of the aptamer were exposed to the dopamine column. The dots represent the collected data while the gray line represents the fit to Equation 17 accounting for the specific and nonspecific binding. The black line represents the specific aptamer binding and the inset represents the linearity between the observed and calculated values.

However, additional methodological considerations also confounded the interpretation of these results as attachment of dopamine through its amine to the column matrix suggests observed affinity values were a reflection of affinity for the functionalized 
dopamine which would have altered chemical properties rather than the free neurotransmitter (Mannironi et al., 1997). Therefore, while displaying a decent affinity for the dopamine column the usefulness of this aptamer for sensing dopamine on its own was still suspect. This, coupled with a fairly large discrepancy between the dissociation constants observed with the Oligreen studies of the unlabeled DNA aptamer and the dissociation constants suggested by the fluorescein labelled form of the DNA aptamer, an approximate 8 fold difference in affinity, highlighted the need to quantify the binding affinity using alternative techniques.

Similar column studies were used to demonstrate that the DNA fluorescein labelled form of the B12 aptamer was able to bind to B12 functionalized column (SEE Appendix Figure 1).

\section{Quartz crystal microbalance}

To address the issue of the affinity of the dopamine DNA aptamer for free dopamine, studies of the aptamer's affinity for dopamine were undertaken using a quartz crystal microbalance. To accomplish this, a thiolated version of the aptamer was produced for chemisorption to the gold electrode of the quartz crystal. Deposition of the aptamer was monitored as a decrease in the oscillation frequency of the quartz crystal over time in the presence of the thiolated aptamer (Figure 2.10). 


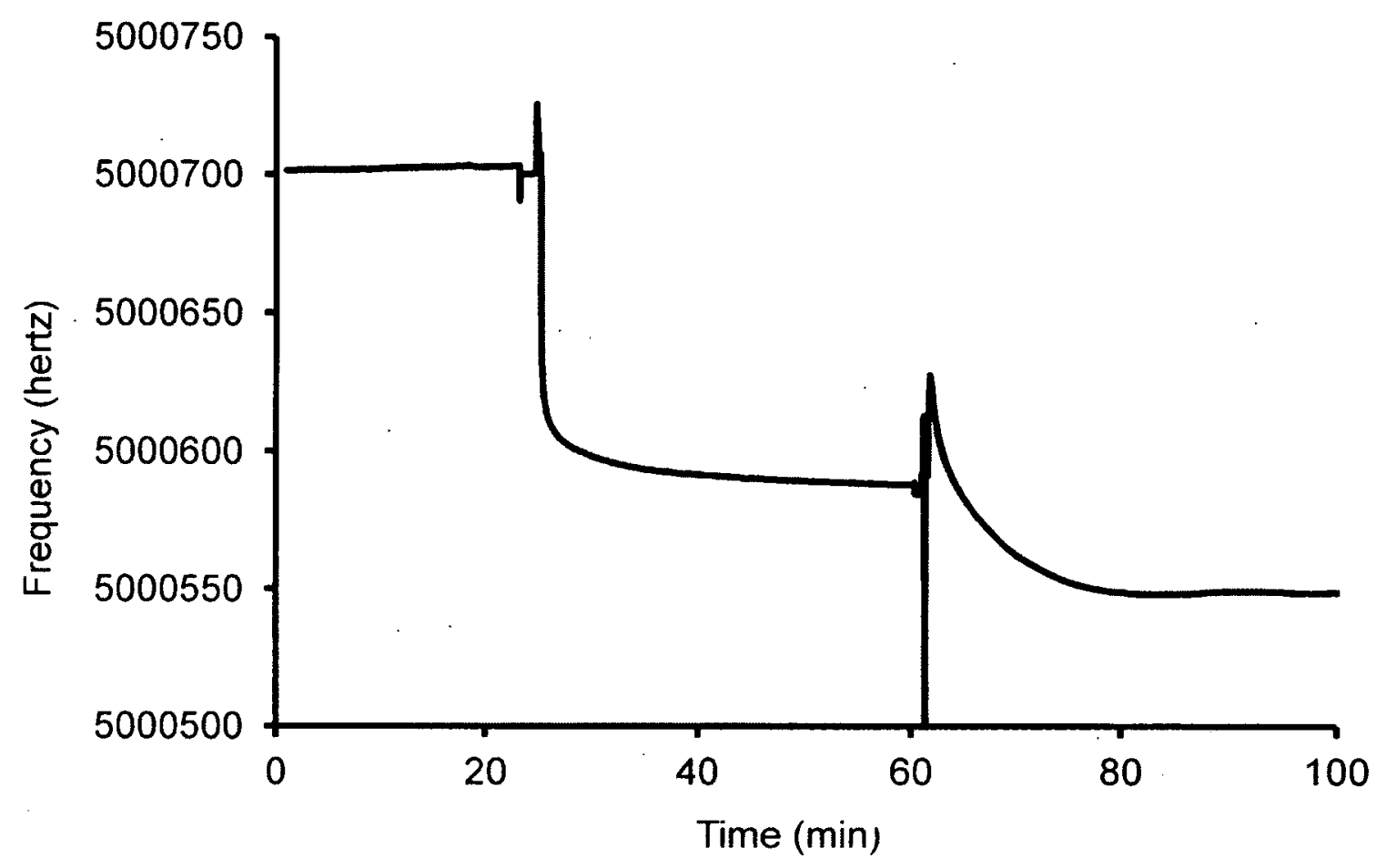

Figure 2.10. Chemisorption of the thiolated aptamer on the gold electrode pad of the QCM crystal. Decrease in frequency at which the QCM crystal vibrated was used to monitor the deposition of the thiolated aptamer by introducing two successive incubations of a $0.4 \mathrm{mM}$ aptamer solution in buffer.

Dopamine binding studies were carried out by initially allowing the aptamer functionalized crystal to equilibrate in buffer followed by the introduction of successively increasing concentrations of dopamine ranging from $10^{-8}$ to $10^{-5} \mathrm{M}$ (Figure 2.11). The aptamer functionalized quartz crystal was found to be unresponsive to dopamine concentrations in this range. As seen in Figure 2.11 the frequency which 
should have decreased with the binding of dopamine to the aptamer remained relatively constant with increasing concentrations of dopamine. The decrease in frequency observed with $4.4 \mu \mathrm{M}$ dopamine at about 170 minutes in this representative plot was attributed to the introduction of an air bubble into the flow cell.

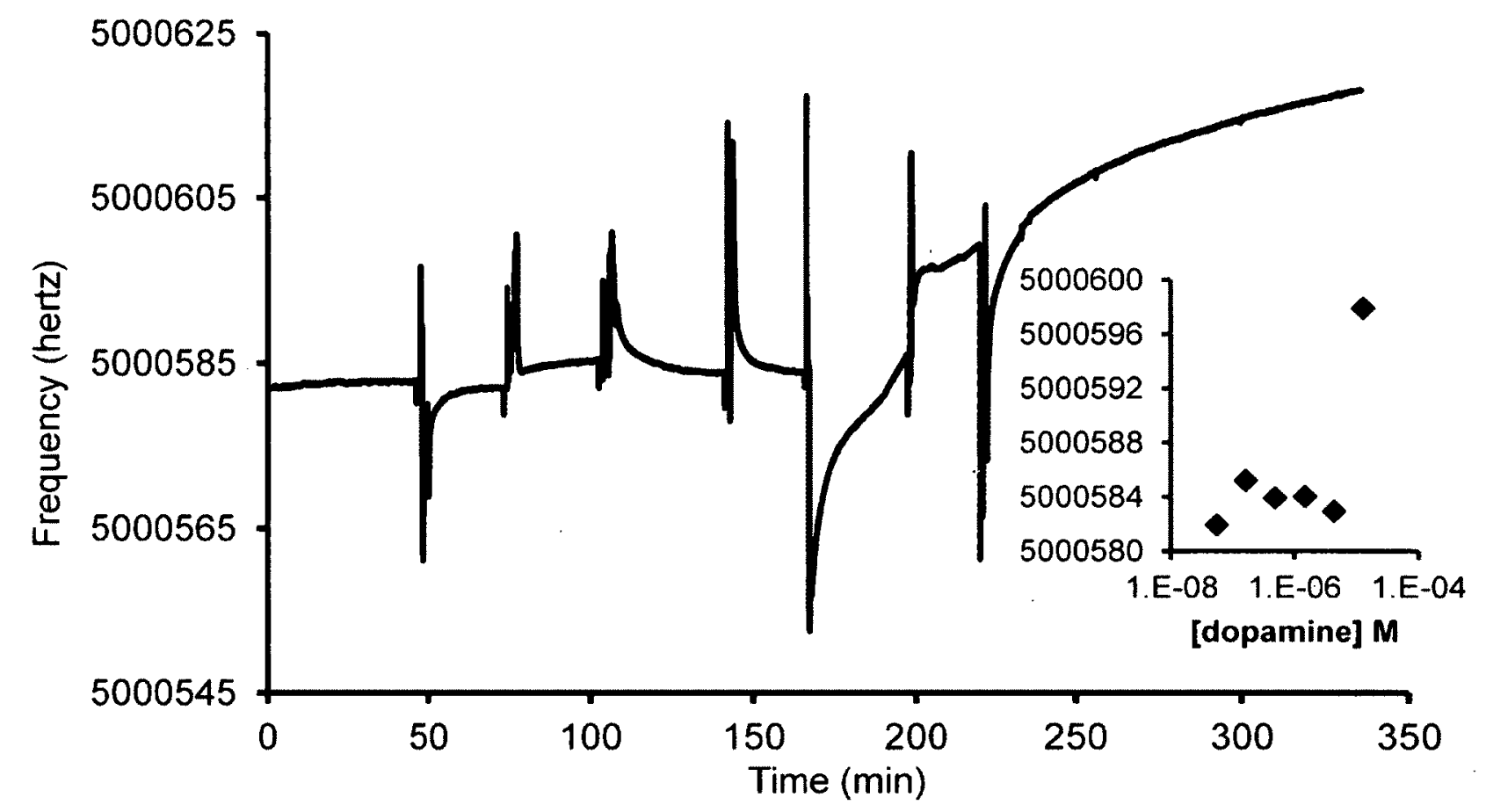

Figure 2.11. Quartz crystal microbalance study of the potential binding between the aptamer and dopamine. The frequency of the aptamer functionalized crystal was monitored as increasing concentrations of dopamine were introduced to the flow cell $(0,0.05,0.16,0.49,1.6,4.4$ and $13 \mu \mathrm{M})$. The insert depicts a plot of the equilibrium frequency plotted against dopamine concentration. 
To determine whether the DNA may not be forming a proper receptor structure due to an inability to leave the laying down phase of the absorption process (Schwartz, 2001) the crystal was back filled with 6-mercaptohexanol (SEE Appendix Figure 2) to provide a organic monolayer of similar length to the thiolated spacer used to modify the aptamer for attachment to the resonator.

Examination of the new surface in the presence of dopamine at relatively low and extremely high concentrations suggested that the introduction of the 6mercaptohexanol organic monolayer was unable to induce quartz crystals responsiveness to dopamine (SEE Appendix Figure 3).

This suggested several possibilities as to why the aptamer functionalized crystal was unresponsiveness to dopamine. The first was that the functionalization of the aptamer to the quartz crystal was disruptive to the structure of the aptamer resulting in an inability to form the proper tertiary structure for dopam:ne binding (Cho et al., 2009).

Alternatively, as previously stated the sensitivity of the quartz crystal microbalance is known to be on the magnitude of nanogram to microgram per $\mathrm{cm}^{2}$, and there have been few successful studies involving the detection of small molecules which are not functionalized in some way as to amplify the signal associated with their binding (Cooper and Singleton, 2007). However Carmen et al., (2004) were able to show such a signal with glucose and its receptor attributing the strong signal to a conformational change in the receptor induced by the binding of the target. As most aptamers are thought to undergo conformational changes in response to ligand binding (Hermann 
and Patel, 2000) the lack of signal produced by the quartz crystal microbalance suggests that if the dopamine was able to bind to the aptamer the relative change in mass was insufficient to produce a change in the frequency of the crystals vibration and also suggests a lack in conformational change of the aptamer upon ligand binding.

In previous studies of the DNA homolog of the B12 aptamer, there was some indication that the DNA sequence had some affinity for the B12 column. To further investigate this phenomenon, a similar study was performed by attaching the thiolated DNA homolog of the RNA B12 aptamer to a quartz crystal and introducing increasing concentrations of B12 to the flow cell (Figure 2.12). Similarly to the DNA dopamine aptamer, the quartz crystal containing the DNA version of the B12 aptamer was found to be unresponsive to $\mathrm{B} 12$ over a wide range of concentrations. Whether the lack in responsiveness was a result of improper folding associated with deposition or a lack of sensitivity of the system was initially unclear. 


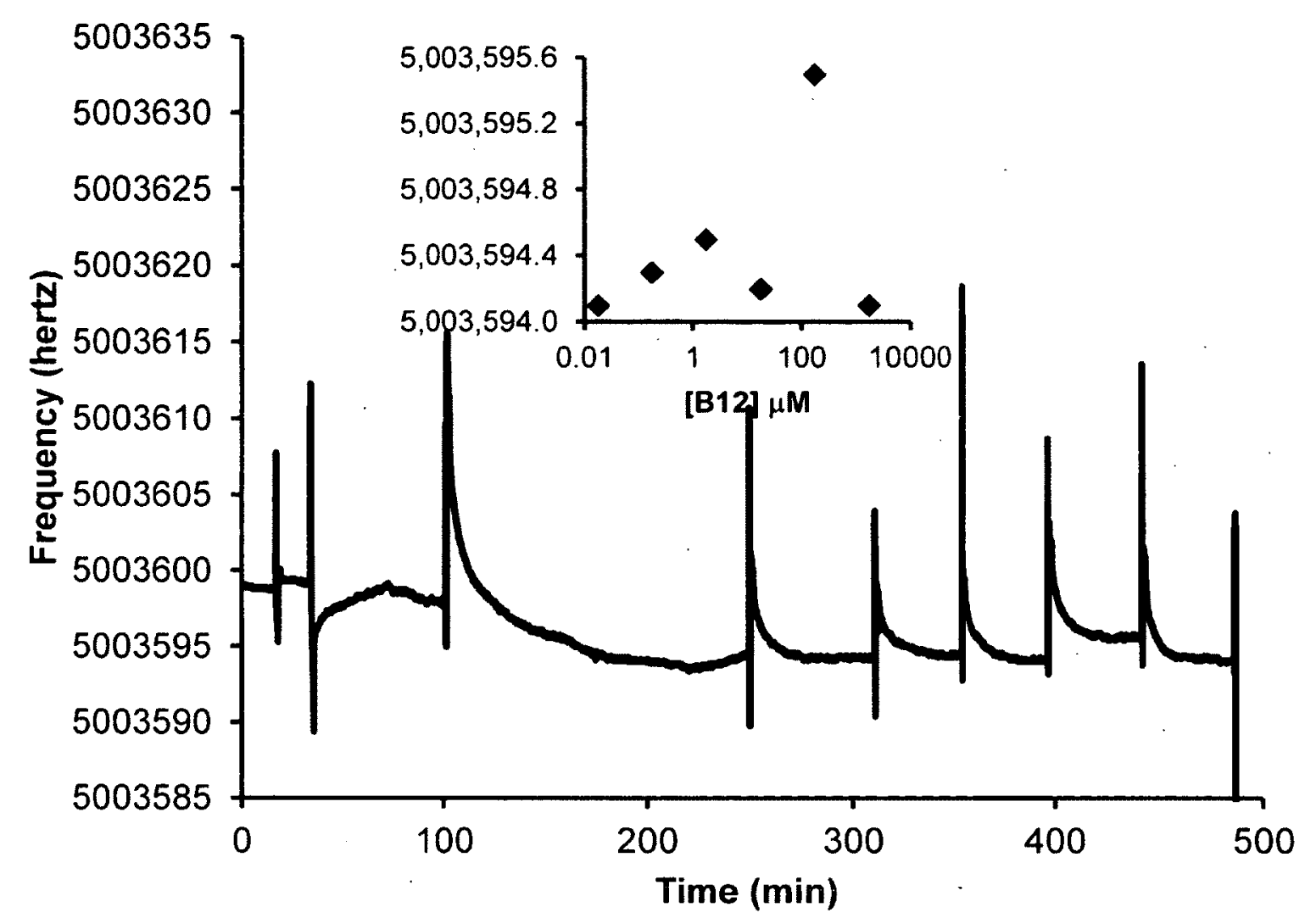

Figure 2.12. Quartz crystal microbalance study of the potential binding between the DNA B12 aptamer and increasing concentrations of B12. The initial three equilibrations corresponded to buffer, followed by the introduction of $0.02,0.2,2,20,200$ and 2000 $\mu \mathrm{M} B 12$. The inset plot represents resting potential versus concentration of B12. 


\section{Isothermal titration calorimetry}

To assess the interaction between dopamine and the unmodified DNA aptamer studies of the aptamers affinity for dopamine were undertaken using an isothermal titration calorimetry instrument.

Initially, dopamine was sequentially added in $12 \mu \mathrm{L}$ amounts into the sample cell containing $1.4 \mathrm{mLs}$ of the $20 \mu \mathrm{M}$ aptamer solution producing a dopamine concentration range from 4 to $38 \mu \mathrm{M}$ corresponding to a molar ratio range between $0.2-2$ for dopamine and the aptamer. The dopamine additions produced a change in heat characterized by an initial sharp endothermic peak followed by a broader exothermic response. This resulted in a net exothermic change associated with the mixing of the two solutions but did not indicate that any of the observed change was associated with a binding event between the aptamer and dopamine (Figure 2.13).

To account for any changes associated with the diffusion of the dopamine in the buffer, a control run of dopamine under the same conditions was performed (Figure 2.14). This indicated that the diffusion of dopamine in the buffer produced a very small exothermic change which did not account for the broader exothermic peak associated with the addition of dopamine to the aptamer solution (Figure 2.13). 

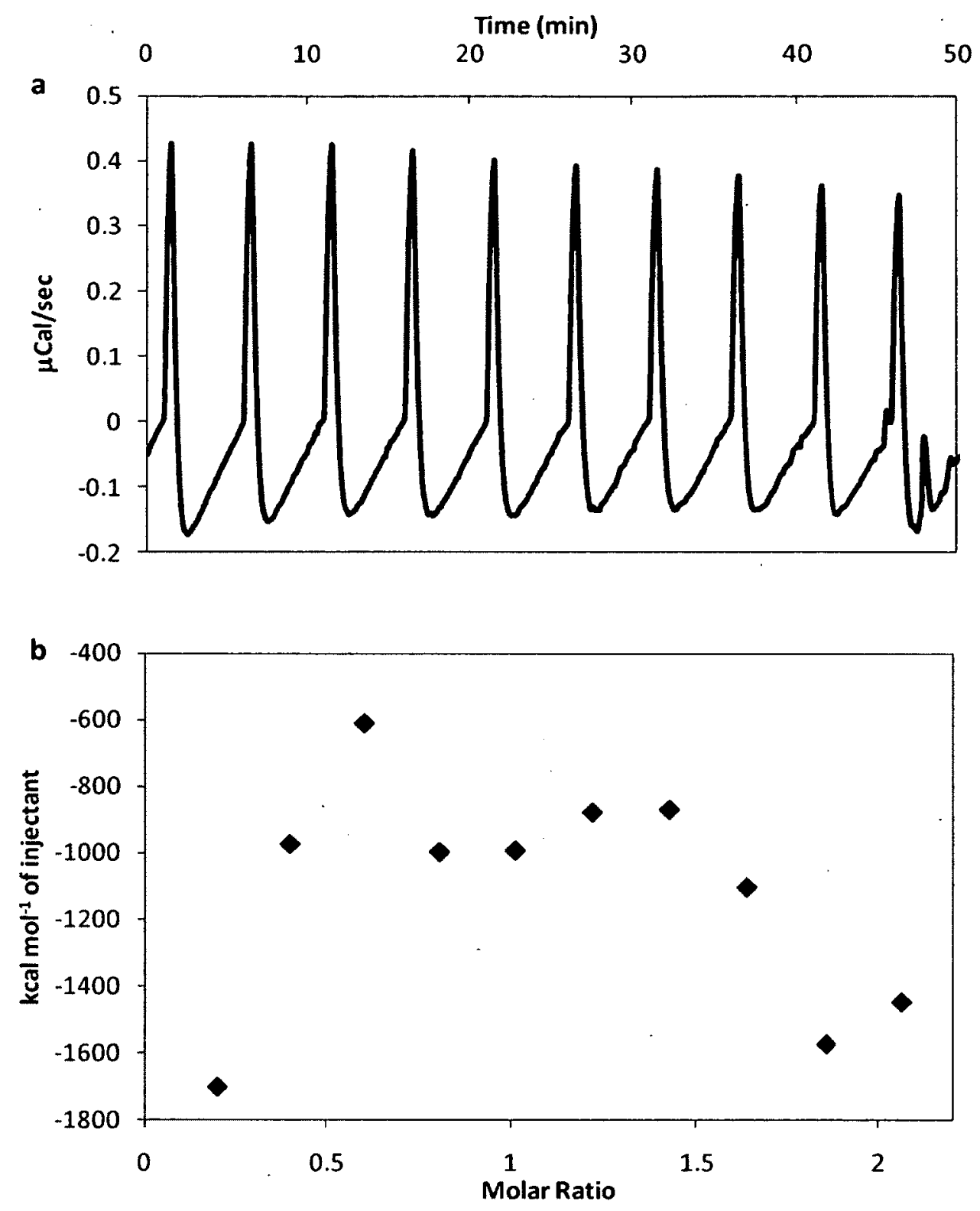

Figure 2.13. Isothermal titration microcalorimetric (a) raw data for the analysis of potential dopamine and aptamer interactions using ten $12 \mu$ injections of $0.47 \mathrm{mM}$ dopamine into $1.4 \mathrm{mLs}$ of $20 \mu \mathrm{M}$ dopamine aptamer and (b) the associated integrated heat plot. 

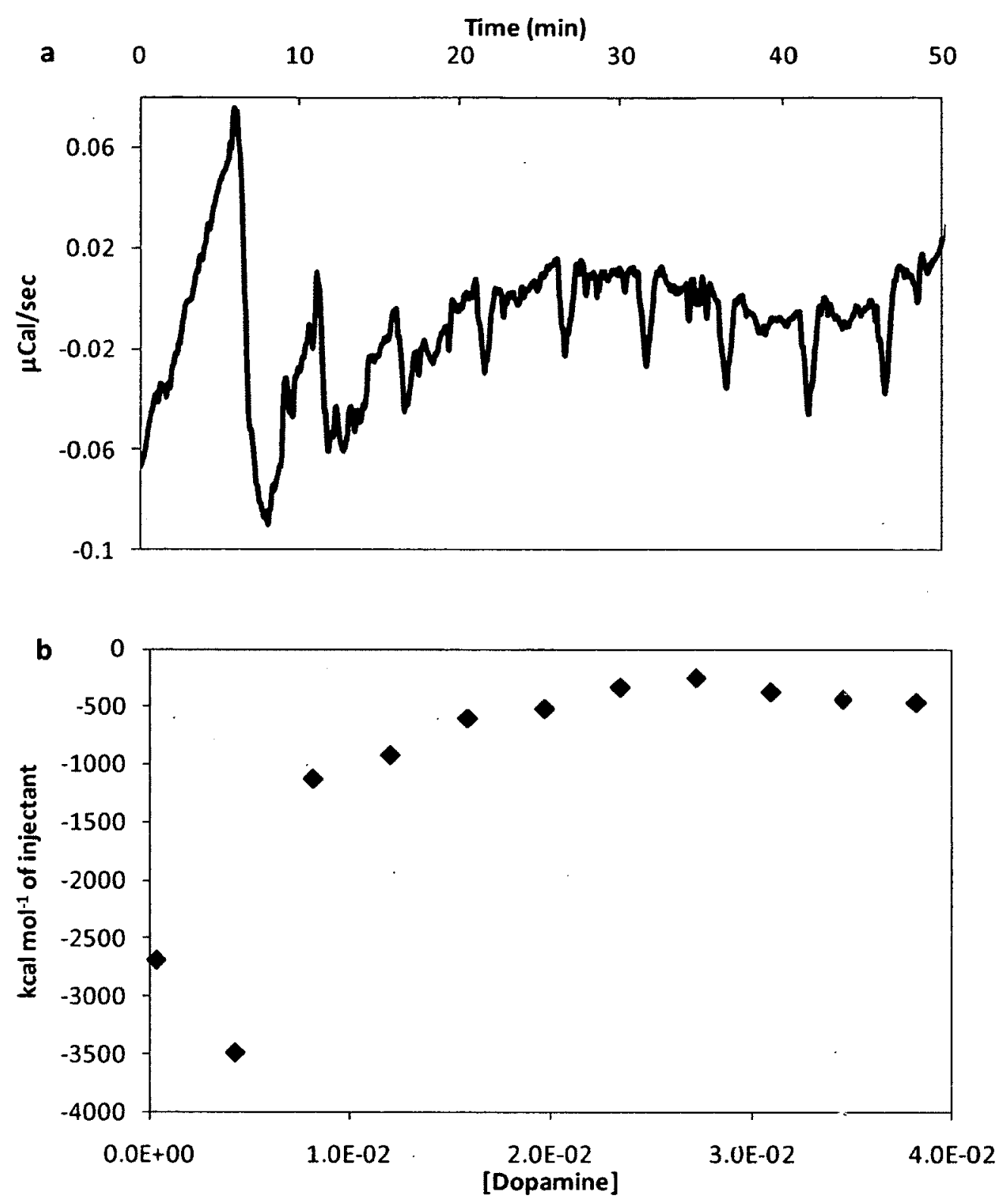

Figure 2.14. Isothermal titration microcalorimetric analysis of the (a) raw data profile for dopamine diffusion into buffer using ten $12 \mu \mathrm{l}$ injections of $0.47 \mathrm{mM}$ dopamine and (b) the associated integrated heat plot. 
To increase the sensitivity of the assay, the injection volumes were adjusted to $6 \mu \mathrm{L}$ in order to reduce possible interference which may of resulted from the mixing of the buffers as their preparation involved the addition of $0.02 \%$ ascorbic acid last, maximizing the ability of ascorbic acid to keep dopamine in the reduced state, resulting in slight differences in the ascorbic acid concentration between the two solutions. The effective assay range of the dopamine concentration was also increased from a dopamine to aptamer molar ratio range of $0.2-2$ to a range of $0.1-3$ by increasing the number of injections to 30 (Figure 2.15). This produced a similar peak profile to the one observed in with the initial experiment (Figure 2.13). A repeat of this experiment confirmed the continued presence of both the endothermic and exothermic peaks resulting in a net exothermic change (SEE Appendix Figure 4).

To eliminate the possible interference of the discrepancy in ascorbic acid concentrations between the aptamer and dopamine solutions the experiment was attempted in the absence of ascorbic acid (Figure 2.16). The omission of the ascorbic acid resulted in a disappearance of the exothermic peaks associated with the injections. The endothermic peaks associated with the injection of the dopamine were however fairly constant in magnitude indicating that the endothermic change was most likely only associated with the diffusion of the dopamine in the sample. This experiment was repeated to confirm the result (SEE Appendix Figure 5). 

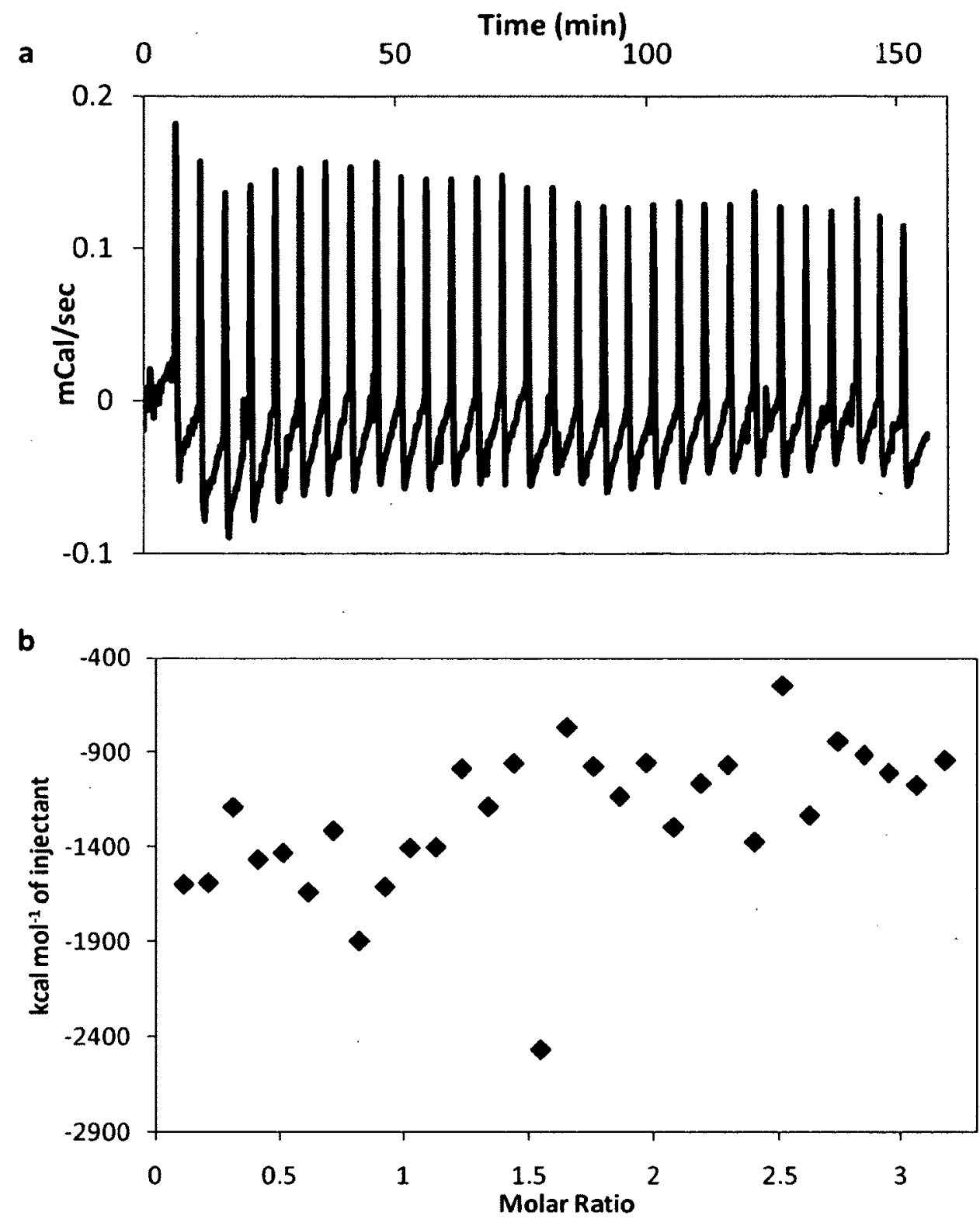

Figure 2.15. Isothermal titration microcalorimetric (a) raw data for the analysis of potential dopamine and aptamer interactions using thirty $6 \mu$ injections of $0.47 \mathrm{mM}$ dopamine into $1.4 \mathrm{mLs}$ of $20 \mu \mathrm{M}$ dopamine aptamer and (b) the associated integrated heat plot. 

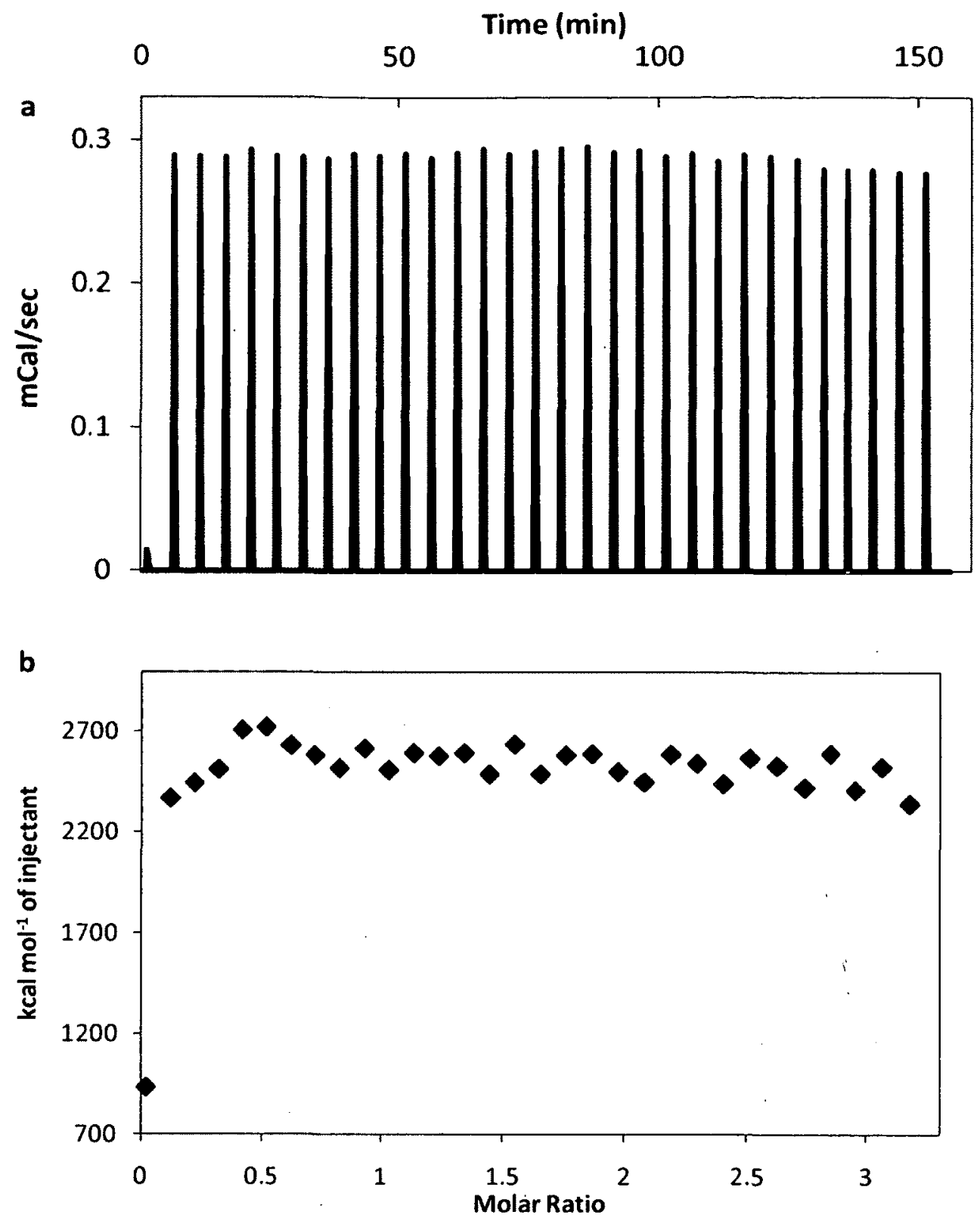

Figure 2.16. Isothermal titration microcalorimetric with ascorbic acid omitted from the buffer (a) raw data for the analysis of potential dopamine and aptamer interactions using thirty $6 \mu \mathrm{l}$ injections of $0.47 \mathrm{mM}$ dopamine into $1.4 \mathrm{mLs}$ of $20 \mu \mathrm{M}$ dopamine aptamer and (b) the associated integrated heat plot. 

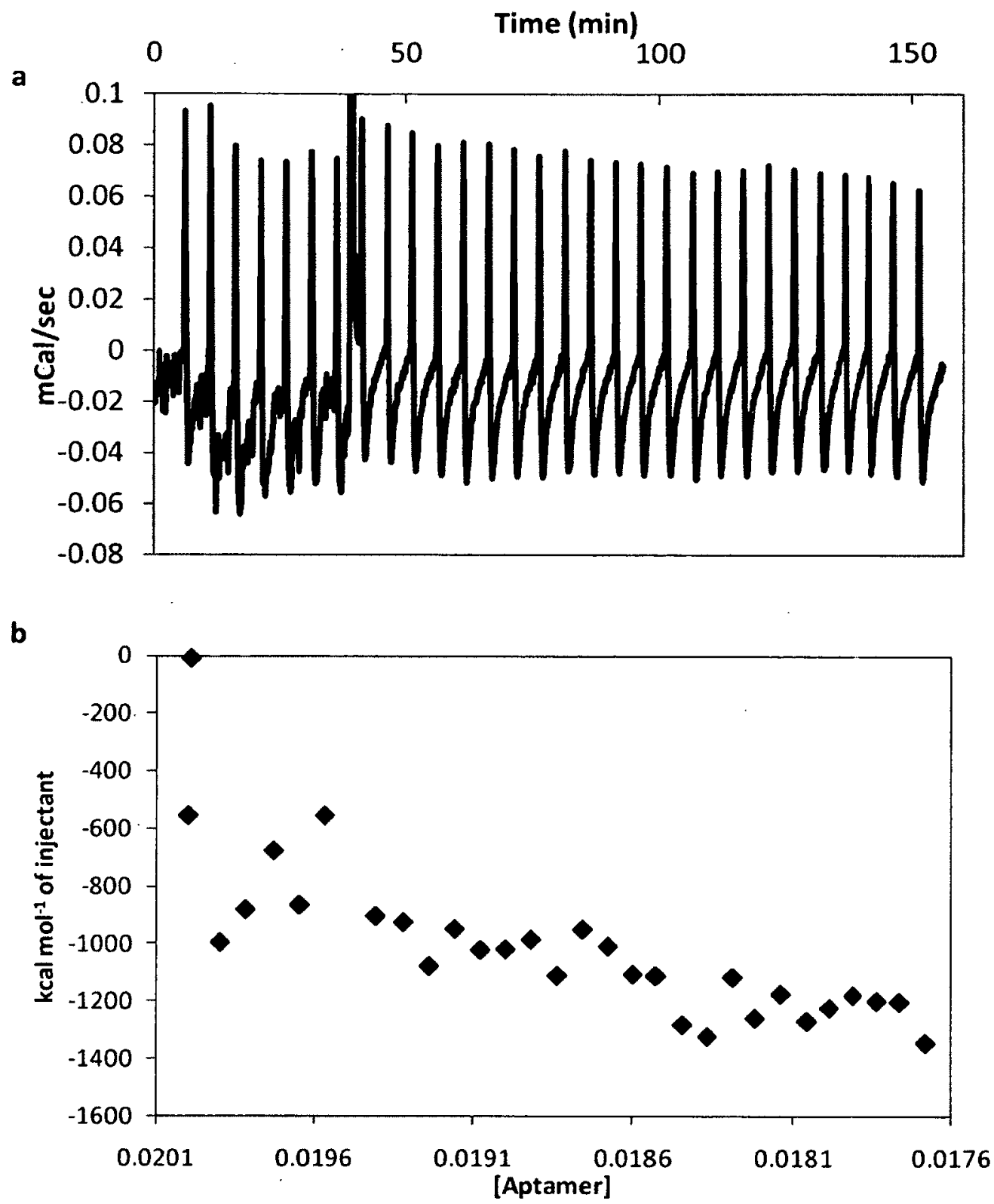

Figure 2.17. Isothermal titration microcalorimetric analysis of the (a) raw data profile for buffer addition to the aptamer using thirty $6 \mu \mathrm{l}$ injections of buffer into $1.4 \mathrm{mLs}$ of 20 $\mu \mathrm{M}$ dopamine aptamer and (b) the associated integrated heat plot. 
To examine what part of the signal might have been associated with the dilution of the aptamer, the experiment was repeated using injections of the buffer alone (Figure 2.17). This indicated that the majority of the signal associated with the previous experiments was attributable to the diffusion of dopamine in the aptamer sample. The lack in change of the integrated heat profiles with change in dopamine concentration suggested that this technique was unable to detect the binding of dopamine and the aptamer. This suggested that the aptamer did not undergo a significant conformational change when binding dopamine and any changes in the overall energy of the system probably resulted from weaker interactions which are hard to detect, such as hydrogen bonding between the aptamer and the catechol portion of dopamine rather than a reorganization of the aptamers structure resulting from the binding of dopamine.

\section{Gel shift assays}

To further assess the interaction between dopamine and the DNA form of the dopamine aptamer gel shift studies were undertaken using the fluorescein labelled aptamer.

Initially, the aptamer was run on a variety of gels in the absence and the presence of dopamine to determine the optimum conditions for visualizing the difference in migration between the free aptamer and the bound aptamer.: 


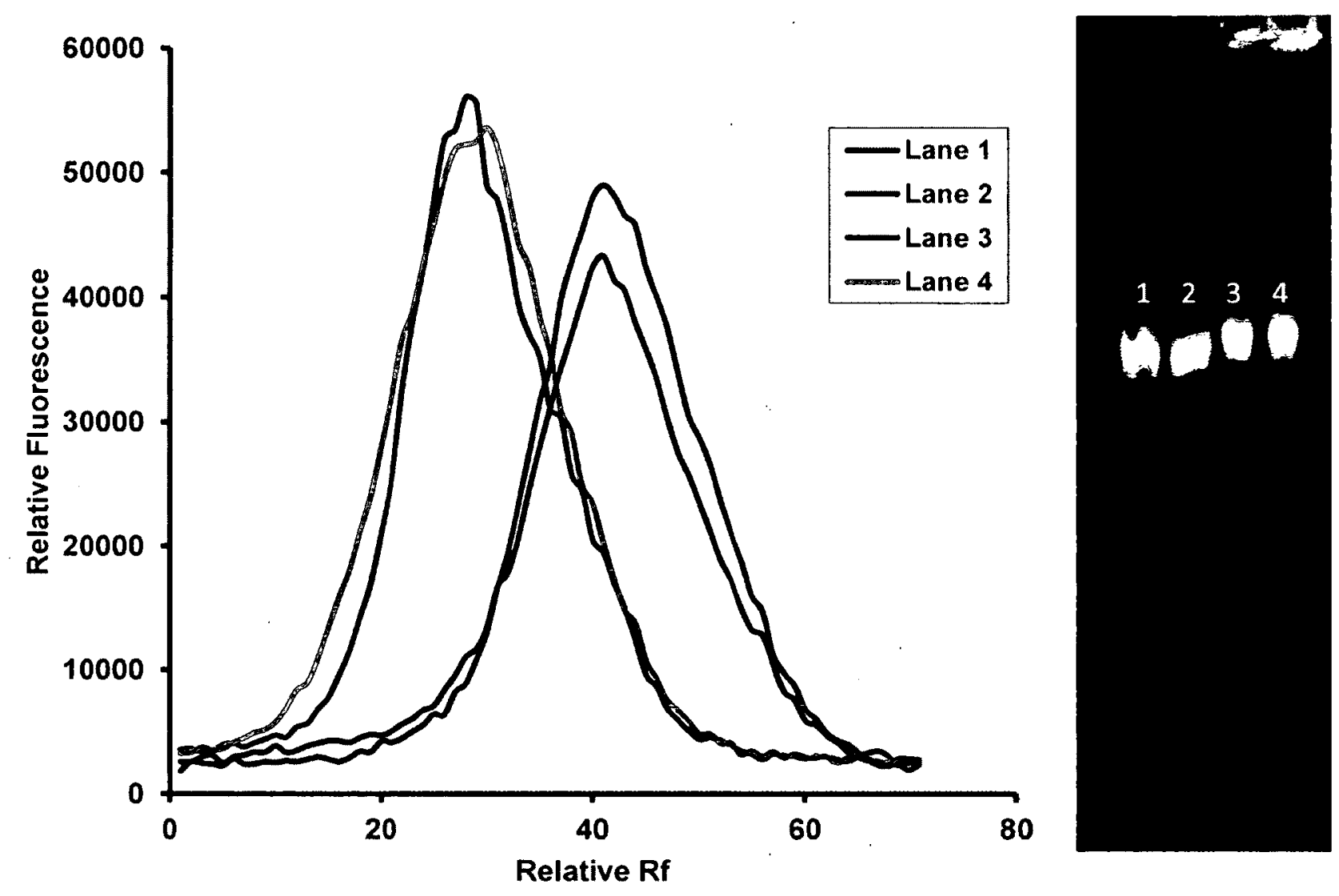

Figure 2.18. Fluorescein labelled DNA homologue of the RNA Dopamine aptamer (4 $\mu \mathrm{M})$ run on a $4 \%$ acrylamide gel at a constant 45 mAmps for 1.5 hours. Lane 1 and 2 contain just the aptamer while lane 3 and 4 were run in the presence of $100 \mathrm{mM}$ dopamine to ensure the presence of the dopamine bound conformation. The relative fluorescence of the fluorescein labelled aptamer was examined with an Alphalmager EC System equip with a $537 / 35 \mathrm{~nm}$ filter. 

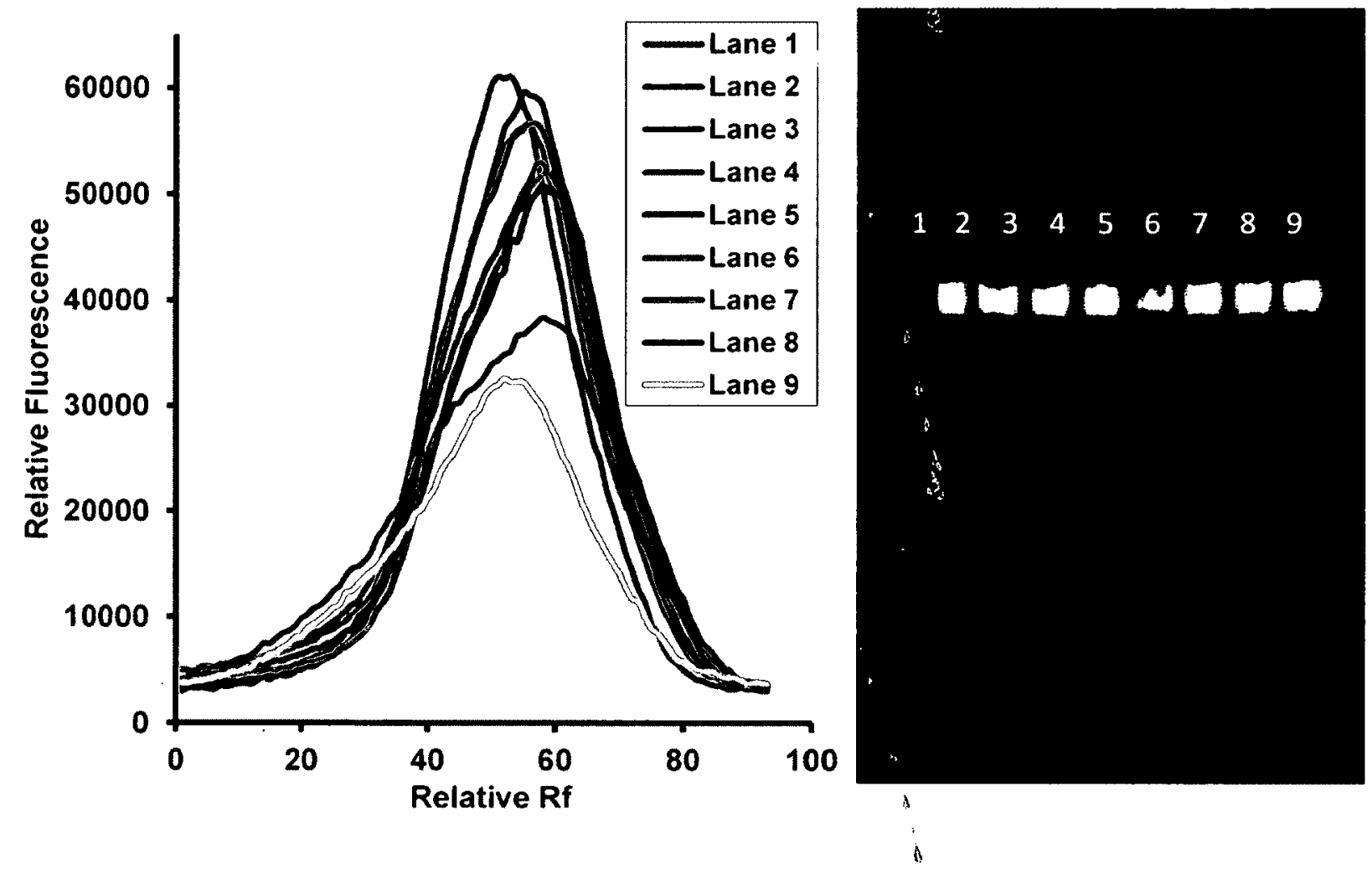

Figure 2.19. Fluorescein labelled DNA homologue of the RNA Dopamine aptamer (4 6

$\mu \mathrm{M})$ run on a $4 \%$ acrylamide gel at a constant 45 riAmps for 1.5 hours. Lane 1-9 contained dopamine of decreasing concentration: $13,1.3 \mathrm{mM}, 130,39,13,3.9,1.3,0.1$ $\mu \mathrm{M}$ and no dopamine. The relative fluorescence of the fluorescein labelled aptamer was examined with an Alphalmager EC System equip with a $5337 / 35 \mathrm{~nm}$ filter. 
Gels run with higher percentages of acrylamide and hence higher cross linking were found to be unsuited for these tests as they forced the aptamer into different conformations which produced multiple running bands in the gels (SEE Appendix Figure 5). Lower concentrations of acrylamide allowed the aptamer to maintain a single band suggesting that the aptamer was able to form a single conformation during its migration (SEE Appendix Figure 7). Further work in the lower acrylamide range suggested that there may be a slight difference in the migration rate between the free aptamer and the aptamer in the presence of dopamine (Figure 2.18). However attempts to determine a dissociation constant for the aptamer and dopamine were unsuccessful (Figure 2.19). This may be attributed the low overall change in mass associated with the formation of the aptamer and target complex. This also supported the evidence of very little or a lack in target induced conformational change associated with the aptamer as was suggested by the isothermal titration calorimetry studies.

\section{Anisotropy studies}

Initially, the DNA form of the RNA dopamine aptamer was labelled with fluorescein and exposed to a broad range of dopamine concentrations (Figure 2.20). In this case no change in anisotropy values was observed with the aptamer. This supported the previous studies which suggested that the aptamer did not undergo a conformational change upon ligand binding. 


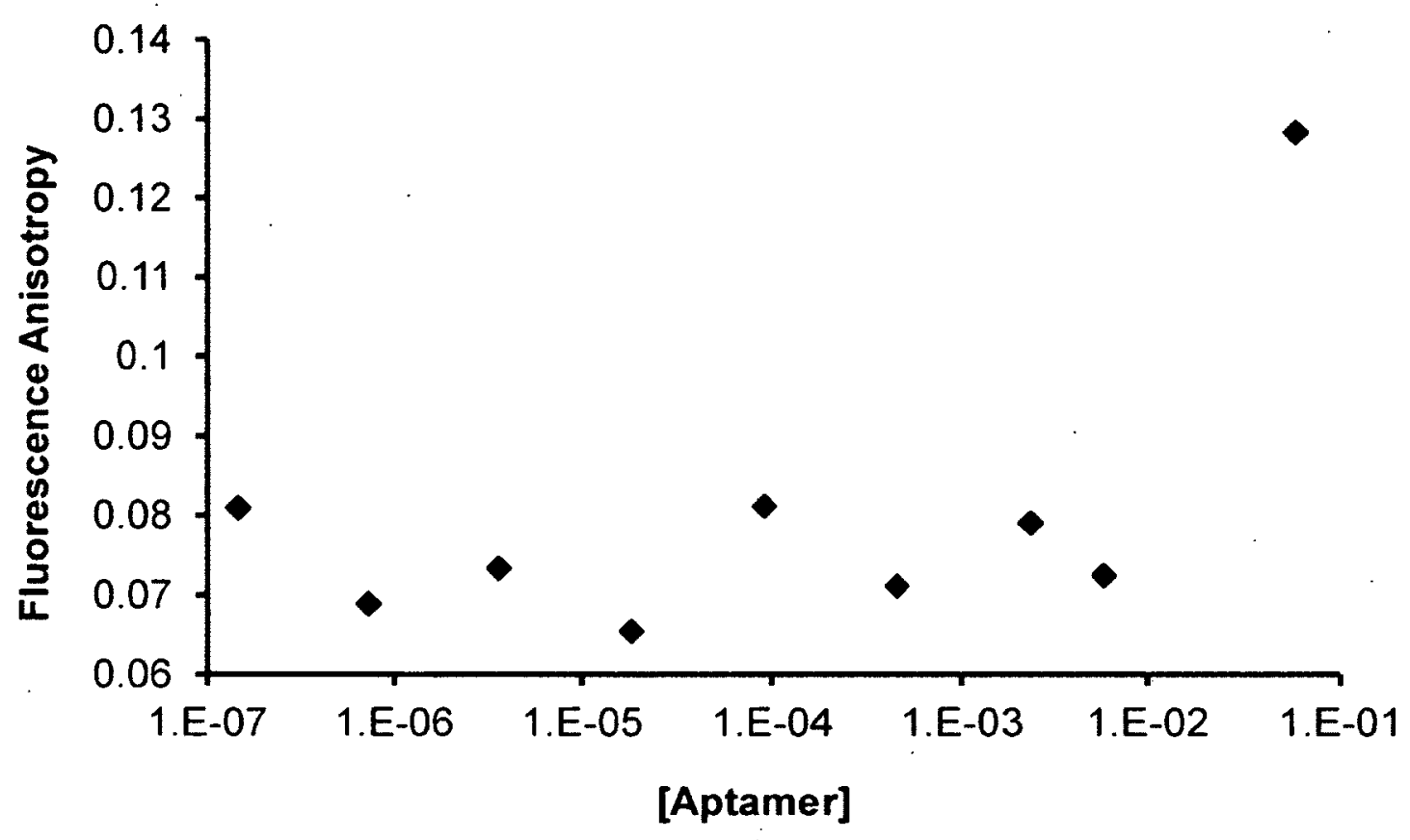

Figure 2.20. Fluorescent anisotropy of the fluorescein labelled DNA dopamine aptamer $(10 \mu \mathrm{M})$ exposed to increasing concentrations of dopamine $(0.14,0.73,3.6,18,91$, $456 \mu \mathrm{M}, 2.28,5.96$ and $59.6 \mathrm{mM})$. 


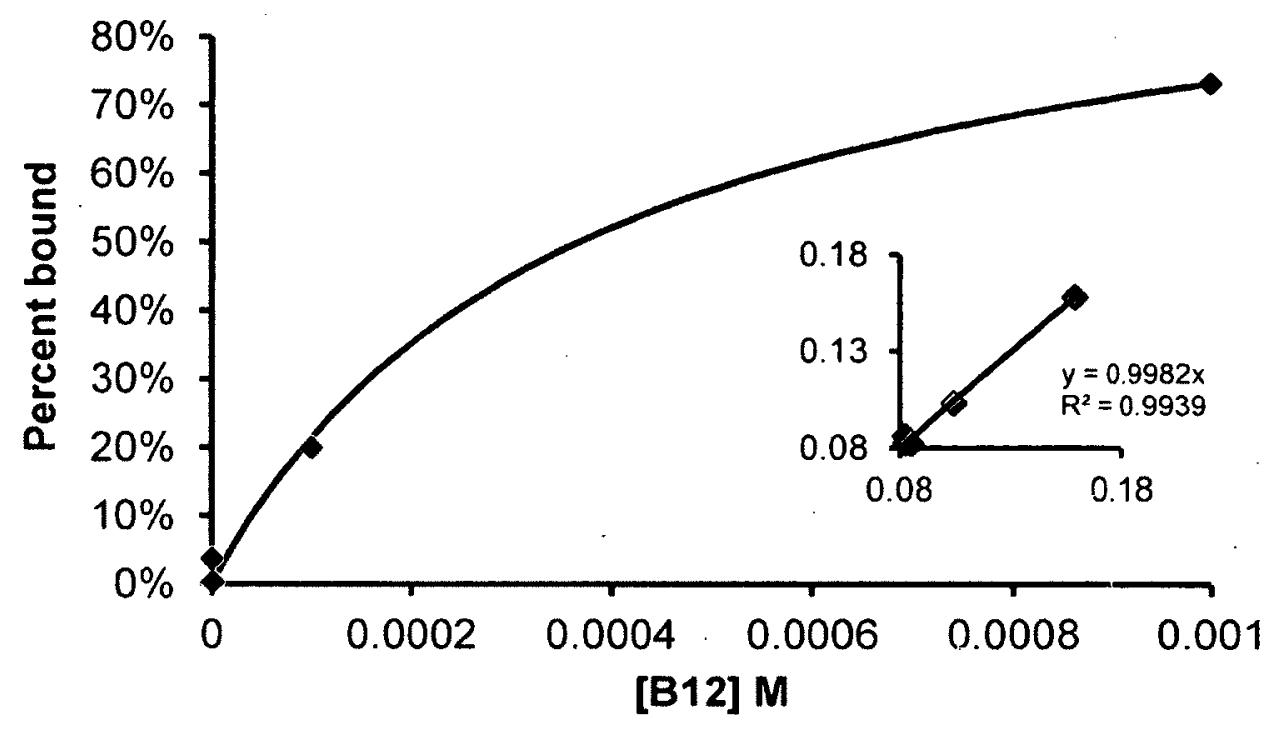

Figure 2.21. Fluorescent anisotropy of the fluorescein labelled B12 aptamer DNA homolog $(10 \mu \mathrm{M})$ exposed to increasing concentrations of $B 12(0.1,1,10,100$ and $1000 \mu \mathrm{M})$. The inset shows the agreement between the observed and the calculated anisotropy values. 


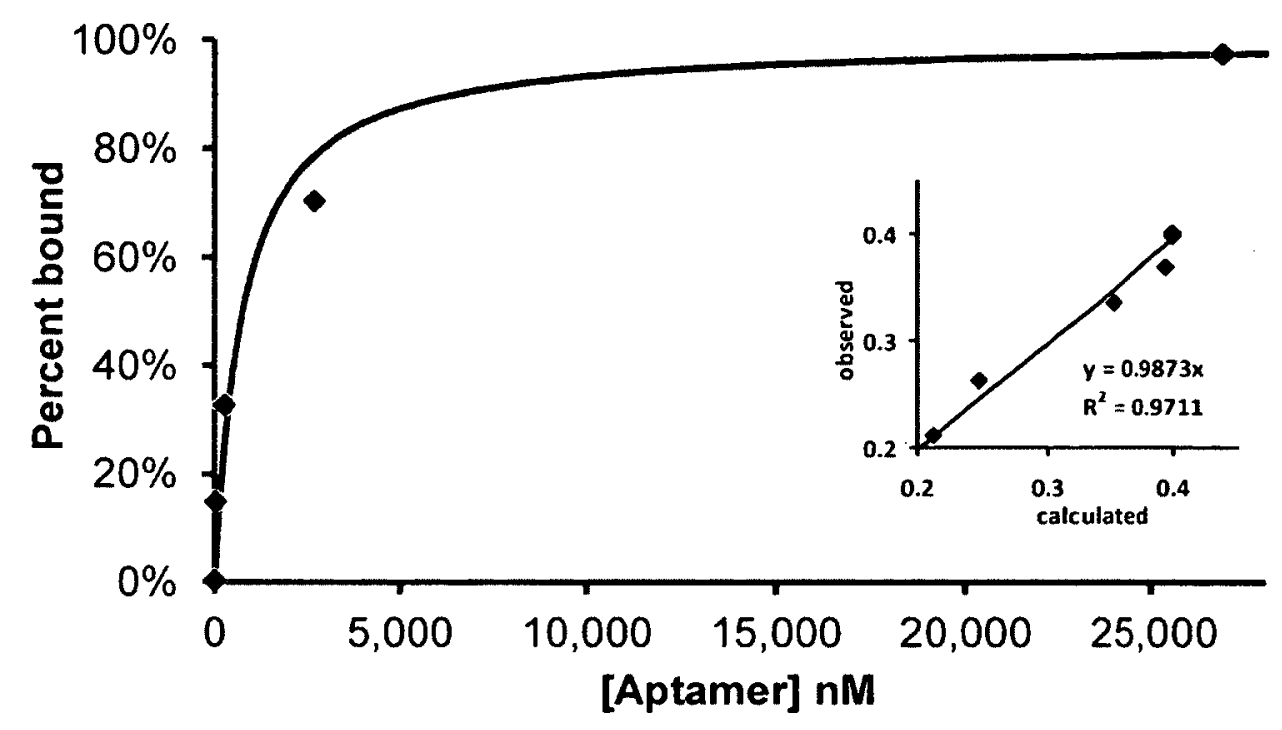

Figure 2.22. Fluorescent anisotropy binding curve for the DNA form of the RNA dopamine aptamer and dopamine. Anisotropy values were collected using $0.1 \mu \mathrm{M}$ dopamine which was exposed to increasing concentrations of the aptamer $(0.27,2.7$, $27,270,2700,27000 \mathrm{nM})$. The inset shows the agreement between the. observed and the calculated anisotropy values. 
<smiles>NCCc1ccc(O)cc1</smiles>

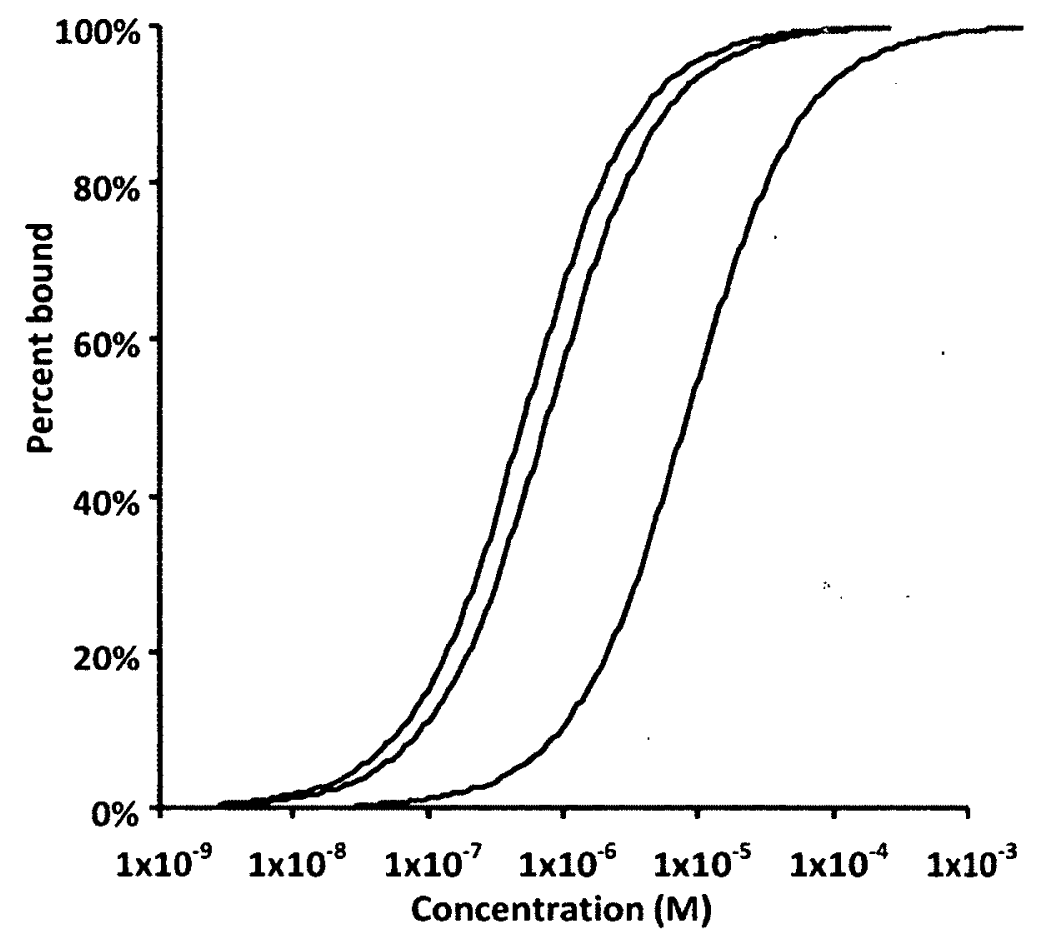

Figure 2.23. Assessment of the specificity of the DNA homolog. Comparison of the binding curves of DNA1 with dopamine (center) and two similar catechols, norepinephrine (left) and tyramine (right) indicated that the DNA aptamer has similar affinity for norepinephrine and dopamine but a much lower affinity for tyramine. 
This also demonstrated that the change in the aptamer's rotational momentum which resulted from dopamine as a small molecule (MW 153.18) binding to the larger aptamer receptor molecule (MW 18206.5) was insufficient to allow determination of the dissociation constant using the fluorescein labelled aptamer.

A similar study with the DNA form of the RNA B12 aptamer suggested that this system was sensitive enough to be used in that instance (Figure 2.21). However the study indicated that the affinity of the DNA form of the B12 aptamer was severely compromised $(370 \mu \mathrm{M})$ when compared to the binding affinity of the original RNA form (88 nM; Lorsch and Szostak, 1994).

While the fluorescently labelled aptamer was unsuccessful in determining the interactions between the aptamer and dopamine, dopamine binding studies were continued using dopamine as the fluorophore (Hoss and Smiley 1977). The results of these studies suggested that this methodology was appropriate for determining the interaction between the aptamer and dopamine (Figure 2.22) allowing for the determination of a binding constant between the aptamer and the ligand of $0.7 \mu \mathrm{M}$ (Table 2.1).

To compare the specificity of the DNA aptamer with the specificity reported for the RNA aptamer, further studies were conducted with norepinephrine and tyramine (Figure 2.23; SEE Appendix Figure 8 \& 9), which share the fluorescent properties of dopamine. (Hoss and Smith, 1977; Hélène et al., 1971). These studies indicated that while the DNA homologue of the RNA aptamer had increased specificity for the 
dopamine relative to RNA aptamer, its selectivity towards dopamine had been altered as the DNA aptamers affinity for norepinephrine was found to be similar or slightly better than for its affinity for dopamine (Kd $0.4 \mu \mathrm{M}>0.7 \mu \mathrm{M})$. The aptamer was also observed to bind tyramine with a binding constant of approximately $7 \mu \mathrm{M}$ although this may be higher as the higher end of the binding curve was not observed. To examine if the DNA form of the dopamine aptamer was binding dopamine in a similar manner to the RNA aptamer, a number of sequences were produced which altered the nucleotides associated with dopamine binding of the RNA aptamer (Table 2.1). These studies demonstrated that conserved nucleotides of the RNA aptamer were also prerequisites for the binding of the DNA form of the aptamer to dopamine.

\section{Discussion}

The ability to replicate the functionality of RNA with sequentially equivalent but the more biologically and chemically stable DNA molecules presents an opportunity to improve nucleotide based nanotechnologies. This stabilization would allow access to the wealth of RNA aptamers and RNA biological machinery, for use in DNA based nanotechnologies. Therefore, a better understanding of and ability to identify sequences and secondary structures that may possess similar properties and functions across both polymer types could be very valuable. Towards that end, we examined the properties of DNA homologs of two distinctly structurally different RNA aptamers, one 
for dopamine (Figure 2.1; Mannironi et al., 1997) and the other for B12 (Figure 2.2; Lorsch and Szostak 1994; Sussman et al., 2000).

The B12 RNA aptamer has been described as a complex pseudo knot which binds B12 at the intersection of a complex trihelical structure, providing a binding surface for the molecular recognition of B12 (Figure 2.2; Sussman et al., 2000). This has led to the comparison of this aptamer to complex biological receptors where the whole structure contributes to maintaining the ligand binding interface. In contrast, the more commonly observed binding motif associated with aptamer and small molecule interactions, involves the binding of the targets to binding pockets which are structurally supported by double helical portions of the molecules (Sussman et al., 2000). A typical example of this is the cocaine aptamer which has a binding pocket that is found at the $t$ junction of two stemloops and a hybridized section between the $3^{\prime}$ and $5^{\prime}$ ends (Stojanovic et al., 2001). This motif is more reflective of the sort of binding observed with the RNA dopamine aptamer which can be described as a folded dumb bell like structure which forms the dopamine binding domain where the loops of the of the two stemloops intersect (Figure 2.1; Mannironi et al., 1997). The distinction between these two structural motifs probably was the determinant factor in the observed differences in functionality of their DNA homologs.

The conversion of the RNA B12 aptamer to its DNA homolog resulted in a change of affinity of $88 \mathrm{~nm}$ to an observable affinity of approximately $370 \mu \mathrm{M}$. This decrease in affinity of approximately 4200 fold indicated that while the RNA aptamer has been 
called one of best aptamers affinity wise, its DNA homologue has very little affinity for B12. There are several structural features of the RNA aptamer that may have contributed to the almost complete loss of binding affinity associated its conversion to the DNA homolog. The tertiary structure of the B12 RNA aptamer is quite complex and structurally uracil seems to play a role in the maintenance of it. In particular the sequence U23, C24, A25 forms a loop that results from U23 and A25 stacking on one another and forcing C24 out into solution (Sussman et al., 2000). This unusual structure, while not strictly precluding the formation of a similar structure by the DNA homolog, belays the importance of uracil in major structural features of the aptamer. Uracil is also involved in stabilization of the main body of the helical triplex as it is involved in the U15:A13:C29 triplet (Sussman et al., 2000). However similarly to the previously mentioned example whether this interaction would be significantly affected by the introduction of thymine base is debatable. However, the highly twisted tertiary structure does suggest that stabilization of the binding surface may be highly dependent on hydrogen bonding between the bases and sugar hydrogen such as is described with the triplet interaction between G8, C21 and A25 (Sussman et al., 2000). As the bases involved in binding B12 in the RNA aptamer do not include uracil and do not appear to involve any of the ribose portions of the aptamer, reduction in binding affinity between the DNA homolog and B12 most likely relates exclusively to an inability of the DNA sequence to assume a structurally equivalent arrangement when compared to the tertiary structure of the RNA aptamer. 
In contrast, conversion of the dopamine aptamer sequence to DNA did not have a deleterious effect on binding, rather, the observed dissociation constant for DNA1 (0.7 $\mu \mathrm{M}$; Table 2.1; Figure 2.22) was found to be better, by a factor of two, than the previously reported result of the RNA aptamer $(1.6 \mu \mathrm{M}$; Mannironi et al., 1997). To determine if the DNA homolog's affinity for dopamine corresponded to the binding pocket proposed for the RNA aptamer, mutant DNA sequences were constructed that target the region of the two loop structure involved in binding between the RNA aptamer and dopamine. Binding between the RNA aptamer is dependent on five nucleotides which forms the binding pocket and a complementary region within the two loop structure which stabilizes the binding pocket (Figure 2.1). Mutants of DNA1 were prepared by incorporating base substitutions at these key sites (Table 2.1). Binding was abolished in all four mutants, as was observed with similar mutants used to characterize the original RNA aptamer. This suggested that the same bases were involved in the binding of the DNA aptamer to dopamine, consistent with retention of a similar binding site. This additionally suggested that retention of function across both polymer types may be more likely to occur with the simpler ligand pocket binding motif: To further characterize the DNA homolog its specificity for dopamine was also tested by examining its affinity for norepinephrine and tyramine (Figure 2.23). The specificity of the RNA aptamer relates to the catechols portion of the molecule; in elution experiments, norepinephrine was able to displace the aptamer off of a dopamine-agarose column while tyramine was not (Mannironi et al., 1997). Binding studies between the DNA homolog and norepinephrine suggested the aptamer had a slightly higher affinity to 
norepinephrine $(K d=0.4 \mu M)$, but retained its specificity for dopamine relative to tyramine (Kd = $8 \mu \mathrm{M}$; Figure 2.23).

Our findings indicate that the dopamine binding site in the dopamine DNA homolog, presumably created through tertiary interactions between the two stemloops, closely resembles that present in the RNA aptamer. The complementarity between the two stem-loops, as well as the presence of five invariant nucleotides, which have been shown to be prerequisites for the RNA aptamers binding of dopamine, also seem to be a necessity for binding between dopamine and the DNA homolog. The improvement in dissociation constant in the DNA homolog and altered specificity, however, may imply that differences in the two polymer structures have some subtle effect on target recognition. The structural flexibility which allows DNA to switch between the B-form helices from and the A-form which RNA is restricted to, may account for the observed changes in affinity.

Additionally, the overall mode of binding between the two aptamers may be considered to be altered as there was quite substantial evidence that the RNA aptamer underwent conformational changes in the presence of dopamine based on its susceptibility to cleavage by ribonucleases (Mannironi et al., 1997). This study suggests very little evidence for the induction of conformational changes in the DNA aptamers structure based on gel-shift and isothermal calorimetry studies. More recently, studies using circular dichroism have suggested that some rearrangement occurs which affects the spectrum in the presence of dopamine (Zheng et al., 2011). However, whether this 
results from the DNA varying between different helical forms rather than inducing conformational changes which would affect hydrogen bonding is not explored.

Furthermore, the stability of the overall structure may differ between the two polymer types. Studies of stem-loop stability have demonstrated that alterations in the sugar-phosphate backbone in some cases may produce significant changes in stability. For example, the replacement of loop riboses with $2^{\prime}$-deoxyriboses has been shown to cause a substantial drop in stem-loop stability for certain RNA sequences, potentially due to favourable electrostatic or other tertiary interactions between the 2 -hydroxyls of the loop and the stem structure (Sakata et al., 1990; Antao et al., 1991; Hannoush and Damha, 2001). These factors may contribute to the observed differences between the RNA and DNA versions of this aptamer.

In summary, the different results obtained using these two aptamer systems suggests several considerations for selecting oligonucleotide. systems that may be amenable to functional transfer between RNA and DNA. The observed improvement in dopamine binding of the DNA homolog of the RNA dopamine aptamer relative to the RNA aptamer along with the mutational inactivation of the DNA homolog suggests maintenance of the same putative binding site between these structures. While the essential reduction of B12 binding affinity, attributed to the DNA homolog of the RNA B12 aptamer, to levels which could be considered nonspecific binding suggests a substantial disruption of the B12 binding surface. 
The dopamine binding pocket of the RNA aptamer is thought to result from base pairing between the loops formed by the two stem-loops of its secondary structure. The tertiary structure formed by this Watson \& Crick base pairing between the loops provides the site of the five invariant nucleotides $(G 13, C 14, A 41, G 51, A 52)$ required for dopamine recognition (Figure 2.1; Mannironi et al., 1997). The lack of uracil bases in the binding site made this aptamer a good candidate for conversion. However, the B12 aptamer similarly does not depend on uracil for ligand recognition but possessed a more complex architecture supporting its binding surface motif (Sussman et al., 2000). The higher degree of structural complexity resulted in an inability of the DNA homolog to bind to the B12 ligand similar to other results reported in the literature (Huizenga and Szostak, 1995; Lauhon and Szostak, 1995; Dieckmann et al., 1997). Indeed, the simpler structure of DNA homolog of the RNA dopamine aptamer represents the first example of an aptamer's conversion from RNA to DNA that was able to retain its function. This emphasizes the difficulty associated with direct conversion between RNA and DNA functional oligonucleotides, however it may be easier to convert RNA to DNA due to the flexibility of its phosphate backbone allowing it to assume multiple helical forms (Figure 1.3).

These observations suggest that direct conversion between DNA and RNA functional oligonucleotides may be limited to simpler motifs, which do not require uracil for ligand recognition and are primarily supported by Watson \& Crick base pairing such as those associated with stem-loops. While direct conversion of functional RNA structures into equivalent functional DNA structures may be limited to simple binding 
motifs, more complex structures may be accessible through SELEX like techniques. By producing pools of degenerative DNA sequences of the original RNA sequence a large number of point and multipoint mutations can be introduced into the DNA homolog sequences which may then be screened for the desired function. This process may be able to find DNA sequences which, when slightly altered, are able to form structures which are functional equivalents to the initial RNA sequence.

\section{Conclusions}

A clearer understanding of the relationship between structure and function along with improved methodologies for converting functional RNAs into DNA would represent a valuable expansion of the DNA based nanotechnology tool kit. These studies suggest a general framework for recognizing structural features which may contribute to viable functional conversions between RNA and DNA which could prove highly valuable in the development of stable new nucleic acids tools for basic research, biosensing and therapeutics.

\section{Acknowledgements}

I would like to thank Dr. Philip Johnson and his graduate students Miguel Neves and

Oren Reinstein, Department of Chemistry York University, for their hospitality and assistance with the Isothermal titration calorimetry portion of this study. 
Table 2.1. DNA sequences used to characterize the binding properties of the DNA homolog of the RNA dopamine aptamer. RNA1: original RNA sequence (Mannironi, et al., 1997)

\begin{tabular}{|c|c|c|}
\hline Name & Sequences $^{\mathrm{a}}$ & $K_{d}(\mu M)$ \\
\hline RNA1 & $5^{\prime}$-GUCUCUGUGUGCGCCAGAGACACUGGGGCAGAUAUGGGCCAGCACAGAAUGAGGCCC-3' & 1.6 \\
\hline DNA1 & GG GCGCCAGAGACAC GGGGCAGA A GGGCCAGCACAGAA GAGGCCC-3' & 0.7 \\
\hline DNA2 & 5' -GTCTCTGTGTGCAACAGAGACACTGGGGCAGATA TGGGCCAGCACAGAATGAGGCCC-3' & b \\
\hline DNA3 & 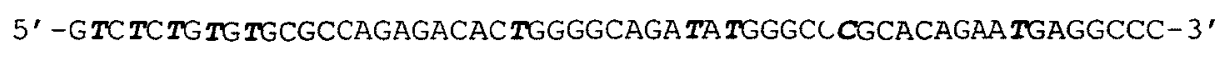 & $b$ \\
\hline DNA4 & 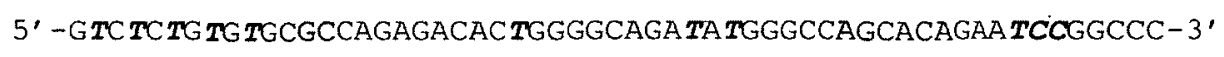 & b \\
\hline DNA5 & 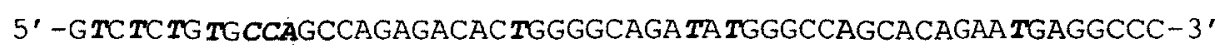 & b \\
\hline
\end{tabular}

${ }^{b}$ no effect 


\section{Chapter 3: Dopamine detection using aptamer}

\section{functionalized glassy carbon electrodes}

Portions of this chapater have been submitted and are under revision for publication in

Bioelectrochemistry 


\begin{abstract}
The detection of dopamine using glassy carbon electrodes suffers from a lack in selectivity toward the neurotransmitter as glassy carbon has been shown to oxidize other catechol-containing neurochemicals and ascorbic acid at overlapping potentials. Several approaches have been employed to improve the selectivity of these electrodes towards dopamine including electrochemical pretreatment and organic monolayer depositions. Here, we characterize glassy carbon electrodes that were initially passivated through trifluoromethylphenyl and nitrophenyl monolayer deposition and then functionalized with a specific DNA dopamine aptamer. These electrodes were more responsive to dopamine than other related cate:hol-containing compounds and additionally they were able to prevent signal interference from other common neurochemicals such as ascorbic acid. Our findings indicate that aptamer functionalization of glassy carbon electrodes may provide an important method for tuning the specificity of electrochemical detection.
\end{abstract}




\section{Introduction}

The ability to specifically and selectively detect biological molecules is of great importance to the understanding of both regular physiological processes and the perturbed metabolic states associated with disease. Aptamers, as receptors of high affinity and specificity, (lliuk et al., 2011) are ideally suited for incorporation into biosensors for the quantification of biological molecules that may offer insight into disease processes (Chen et al., 2011). Dopamine, the neurotransmitter of the dopaminergic nervous system, is of particular interest for specific detection and quantification, as perturbations of the dopaminergic nervous system are involved in the formation of addictive behaviour (Klein et al., 2007), Parkinsonism (Lees et al., 2009) and Schizophrenia (Basile etal., 2002). While the basic mechanisms of these disorders may result from environmental influence, genetic predisposition and molecular processes at the cellular level, the overall physiological expression results from dysfunction of neural communication (Siddique and Tan, 2010; Faludi and Mirnics 2011; Shulman et al., 2011). Therefore, the ability to assess changes in dopamine signalling provides a way of evaluating and identifying problems affecting the functionality of the dopaminergic nervous system.

In the 1970 s, push pull perfusion was introduced for the study of neurotransmitters (Myers et al., 1998). Push pull perfusion involved the cerebral implantation of needles which produced continual influx and efflux of perfusion solution which could then be subjected to HPLC techniques for the detection of neurotransmitters such as dopamine. While the push pull method allowed for multiple types of experiments to be performed 
such as the introduction of drugs directly to the neuroanatomical region under study, (Myers et al., 1998) it also suffered a few problems. The continual pressure of the perfusion has been linked to tissue damage and stress-induced release of dopamine and the continual flow has been observed to result in the induction of neurochemical concentration gradients up to several millimetres proximal to the sampling site (Myers et al., 1998).

To overcome some of the problems associated with push pull techniques, microdialysis was introduced which segregated perfusion solutions from the tissue. While microdialysis techniques reduced tissue interaction, encasing the flow of the perfusion solution behind a dialysis membrane increased the size of the system reducing anatomical spatial resolution and decreasing the sensitivity relative to push pull methods. Both of these techniques, however, suffer from issues with temporal resolution as well as collected samples requiring further processing such as HPLC-based separation and quantification (Kim et al., 2012).

The next step in dopamine detection was the development of dopamine-detecting electrodes by Adams in 1976. With electrodes on the scale of $10 \mu \mathrm{m}, 10$ to 100 times smaller than push pull and microdialysis probes, electrochemical detection of dopamine increased both spatial and temporal resolution in neurochemical anatomical studies (Adams, 1976; Mas et al., 1996). These advantages spurred innovation in dopamine electrochemical techniques, such as the use of different materials like bare glassy carbon electrodes, which can be used to directly detect dopamine (Gonon et al., 1980). 
Bare electrodes, however, suffer from a lack of selectivity towards dopamine that has resulted in their susceptibility to interference from other catechol containing compounds and neurochemicals such as serotonin and ascorbic acid which oxidize at potentials similar to dopamine (Adams, 1976; Gonon et al., 1980; Liu and Wang 1994). To overcome this problem, there has been a lot of work into the modification of these electrodes to improve their responsiveness to dopamine (Downard et al., 1995; DuVal and McCreery, 1999; McCreery, 2008). Electrode modification efforts have ranged from electrochemical pretreatment to increase surface oxides, to the deposition of organic monolayers. Kelly et al., (1999) examined the effect of oxygen containing functionalities on carbon sensor behaviour. Their work demonstrated a relationship between surface charge and specificity for electroactive species. Modification of glassy carbon with a phenylacetate layer was found to allow for effective discrimination between dopamine and ascorbic acid (Downard et al., 1995). Similar modifications using glycine and betaalanine, have demonstrated that surface modification can be used to change the overpotentials of dopamine and ascorbic acid sufficiently for simultaneous detection and quantification (Zhang and Lin, 2001; Zhang and Sun 2001). More recently, graphene oxide electrodes have been produced that reduce the overpotential of uric acid as well allowing for the simultaneous detection of dopamine, ascorbic acid and uric acid (Ping et al., 2012).

Work with organic monolayers has also led to the observation that catecholamine electrode absorption is a prerequisite process for electron-transfer (DuVal and McCreery, 1999). This has been specifically demonstrated with the use of 
trifluoromethylphenyl and nitrophenyl chemisorbed monolayer which prevent absorption and inhibit the oxidation of catecholamines (DuVal and McCreery, 1999).

While the selectivity of carbon electrodes continues to improve, the passivation of the electrodes reported by DuVal and McCreery (1999) suggested a way of producing highly selective surface by passivating the surface with trifluoromethylphenyl and nitrophenyl and then functionalizing the surface with the dopamine aptamer.

In the previous chapter we were able to demonstrate that the DNA form of the RNA dopamine aptamer (Mannironi et al., 1997) retained its ability to bind dopamine (Walsh and DeRosa 2009). The usefulness of this aptamer has already been demonstrated with further studies describing its use in vivo and in vitro for dopamine binding applications. For example Zheng et al. (2011) adapted the aptamer to a detection technique using gold nanoparticles which placed the linear detection range for dopamine from 0.5 to 5 $\mu \mathrm{M}$. This supports the binding affinity $(0.7 \mu \mathrm{M})$ reported in the previous chapter and demonstrated the robustness of the DNA form of the aptamer for adaption to various tasks. The robustness of the DNA homolog was further demonstrated by Holahan et al. (2011) who used it to modify the addictive behaviour of rats through direct injection into the nucleus accumbens. This demonstration of in vivo direct modulation of dopaminergic processes in the nervous system of rats highlights the stability and plethora of potential applications produced by the conversion of the RNA aptamer to DNA. Here, we examine the ability of this aptamer to produce a selective dopamine signal by attaching the aptamer to glassy carbon electrodes which have been passivated 
with trifluoromethyphenyl and nitrophenyl groups. Using square wave (Ramaley and Krauser, 1969) and cyclic voltammetry (Nicholson and Irving, 1964), the aptamerfunctionalized surface was shown to produce a selective surface for dopamine detection.

\section{Materials and Methods}

Glassy carbon electrodes ( $3 \mathrm{mM}$ diameter) were purchased from CHInstruments. Ascorbic acid, cetrimonium bromide, 3,4-dihydroxyphenylacetic acid, diisopropyl ethyl amine, dimethylformamide (DMF), dopamine, fluoboric acid (48\%), 4-nitroanaline, norepinephrine, sodium nitrite, serotonin, 4-(Trifluoromethyl)aniline, o-( $\mathrm{N}$ Succinimidyl)-1,1,3,3-tetramethyluronium tetrafluoroborate (TSTU), and tyramine were purchased from Sigma-Aldrich Canada. L-3,4-Dihydroxyphenylalanine (Ldopa) was purchased from TCl America.

10-Carboxy-decyl-(2-cyanoethyl)-(N,N-diisopropyl)-phosphoramidite Nhydroxysuccinimide ester, and phosphoramidites for DNA synthesis were purchased from Glen research

All electrochemical studies were performed using a CHInstruments $660 \mathrm{c} \mathrm{Ag} / \mathrm{Cl} P \mathrm{Pt}$ electrochemical workstation ( $\mathrm{CH}$ Instruments, Inc. USA). 


\section{DNA Sequences}

DNA homologue of the RNA dopamine aptamer, the $5^{\prime} \mathrm{N}$ denotes the position of the carboxyl modifier used to link the sequence to the electrode surface.

5'-NGTCTCTGTGTGCGCCAGAGACACTGGGGCAGATATGGGCCAGCACAGAATGAGGCCC-3'

Random DNA control sequence for electrochemistry experiments, the $5^{\prime} \mathrm{N}$ denotes the carboxyl modifier used to link the sequence to the electrode surface.

5'-NAAGCCCGGCAGAGTTAGTTCGAACCTAATGCCACAAATGAGACAGGACGCCAATGGG-3'

\section{Electrode preparation}

The diazonium compounds 4-nitrobenzenediazonium and 4-(trifluoromethyl) benzenediazonium, used to modify the glassy carbon electrode, were produced based on the procedure of Dunker et al., (1936). 4-Nitrobenzenediazonium was produced by slowly adding 1 equivalent of $8 \mathrm{M}$ sodium nitrite to 3 equivalents of $2 \mathrm{M}$ 4-nitroanaline in $48 \%$ fluoboric acid, $38^{\circ} \mathrm{C}$. Crystals were filtered, washed with ethyl ether and stored at $-20^{\circ} \mathrm{C}$. 4-(Trifluoromethyl)benzenediazonium was produced by slowly adding 1 equivalent of $8 \mathrm{M}$ sodium nitrite to 3 equivalents of $2 \mathrm{M} \mathrm{4-(Trifluoromethyl)} \mathrm{aniline} \mathrm{in}$ $48 \%$ fluoboric acid, $38^{\circ} \mathrm{C}$. The sample was chilled briefly at $-20^{\circ} \mathrm{C}$, to encourage crystallization, gravity filtered and washed with ethyl ether. The crystals were then stored at $-20^{\circ} \mathrm{C}$. 
The production of the desired diazonium compounds were confirmed using IR spectroscopy (Kazitsyna et al., 1964).

\section{Electrode modification}

Glassy carbon electrodes were polished with coarse silica (60-200 $\mu \mathrm{M}$ diameter) and then fine silica $(0.05 \mu \mathrm{M}$ diameter) suspended in nanopure water. The nanopure water was prepared by mixing deionized distilled $\mathrm{H}_{2} \mathrm{O}$ with activated carbon and filtering into flame sterilized glassware. The electrodes were then washad with nanopure water and dried (McCreery 2008).

Following polishing, the electrode surfaces were modified according to the methods described by McCreery (2008). Initially a single scan $(100 \mathrm{mV} / \mathrm{s})$ between +0.4 to $-0.4 \mathrm{~V}$ using a solution of $0.1 \mathrm{M}$ 4-(Trifluoromethyl) benzenediazonium in acetonitrile was used to deposit a partial coverage of trifluoromethylphenyl groups This was followed by functionalization of the surface using a solutions of $0.1 \mathrm{M}$ 4-nitrobenzenediazonium in acetonitrile with a second scan between +0.4 to $-0.4 \mathrm{~V}$, providing accessible nitrophenyl groups for further modification. The surface passivation was completed with a third scan in between +0.4 to $-0.4 \mathrm{~V}$ with $0.1 \mathrm{M} 4$-(Trifluoromethyl) benzenediazonium in acetonitrile.

Reduction of the nitrophenyl groups to aminophenyl groups was performed by scaning $(100 \mathrm{mV} / \mathrm{s})$ four times between -0.4 and $-1.5 \mathrm{~V}$ in $90: 10 \mathrm{H}_{2} \mathrm{O}$-ethanol and $0.1 \mathrm{M}$ $\mathrm{KCl}$ (Allongue et al., 1997). 


\section{Electrode aptamer functionalization}

Electrodes were functionalized with the DNA homologue of the RNA dopamine aptamer or with a random DNA sequence of equal length which had been prepared using a Mermaid 6 oligonucleotide synthesizer (Bioautomation). Both sequences were prepared with the addition of a 5' Carboxy-N-hydroxysuccinimide ester. Following the synthesis the DNA was deprotected with $0.4 \mathrm{M}$ methanolic sodium hydroxide (4:1 methanol: $\mathrm{H}_{2} \mathrm{O}$ ), for approximately 17 hours at room temperature. This incubation removed the nucleotide protecting groups and the $\mathrm{N}$-hydroxysuccinimide ester leaving the free terminal carboxyl group. The supernatant was dried overnight in the presence of a few crystals of dibasic sodium phosphate. Recovered samples were suspended in $50 \mu \mathrm{L} \mathrm{diH}{ }_{2} \mathrm{O}$, and isolated on a $12 \%$ acrylamide gel.

Isolated samples were resuspended in $\mathrm{H}_{2} \mathrm{O}$ and precipitated with the addition of 2 $3 \mu \mathrm{L}$ of $8 \%(w / v)$ aqueous cetrimonium bromide (Boutorine and Sun, 2005). The sample was centrifuged at $10,000 \mathrm{~g}$ for $2 \mathrm{~min}$ to pelletize the precipitäte and the supernatant was removed. The sample was then dried on the speedvac followed by the addition of $1 \mathrm{~mL}$ of $0.1 \mathrm{M} \mathrm{TSTU}$ in dimethylformamide to solubilize the pellet and activate the $5^{\prime}$ terminal carboxyl group (Wilchek et al., 1994). The sample was shaken until the majority of the pellet was suspended. To this mixture, $1 \mathrm{~mL} 0.1 \mathrm{M}$ diisopropyl ethyl amine in dimethylformamide was added to increase the $\mathrm{pH}$ and increase the reactivity of the aminophenyl groups on the surface of the electrodes. The aminophenyl 
functionalized electrodes were incubated with the DNA samples on a shaker overnight at $37^{\circ} \mathrm{C}$.

\section{Aptamer electrode electrochemical characterization}

DNA functionalized electrodes were tested for their responsiveness to dopamine and a variety of catecholamines using circular voltammetric(Nicholson and Irving, 1964), scan rate $100 \mathrm{mV} / \mathrm{s}$, and square wave scans (Ramaley and Krauser, 1969) using a pulse frequency of $15 \mathrm{~Hz}$, amplitude $25 \mathrm{mV}$ and $4 \mathrm{mV}$ increments, between 0 and $0.8 \mathrm{~V}$. Typically ligands were freshly prepared in $5 \mathrm{mM} \mathrm{MgCl}_{2}, 0.5 \mathrm{M} \mathrm{NaCl}$ and $50 \mathrm{mM}$ Tris $\mathrm{HCl}$ $\mathrm{pH}$ 7.4. Electrodes were cleaned between samples via immersion in buffer containing

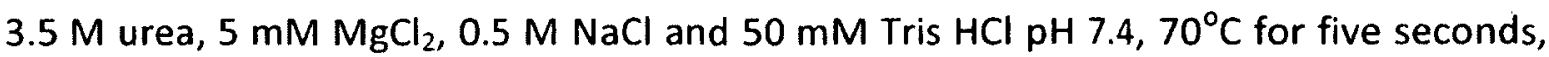
followed by washes with $95 \%$ ethanol and distilled $\mathrm{H}_{2} \mathrm{O}$.

\section{Results and Discussion}

Preparation of the aptamer functionalized electrodes was based on the previous observed passivation of glassy carbon electrodes to catecholamine oxidation using electrochemical organic monolayer deposition (DuVal and McCieery, 1999; Figure 3.1). The deposition of organic monolayers on glassy carbon electrodes using diazonium ion is known to be fairly efficient but requires multiple scans (McCreery 2008). This allowed us to change the diazonium ion between 4-(Trifluoromethyl)benzenediazonium and 4nitrobenzenediazonium between successive scans (Figure 3.1). Consistent with studies on the deposition of the individual molecules on glassy carbon electrode surfaces, the 
mixture produced the characteristic inhibitory decrease in peak current associated with the formation of the organic monolayer (Allongue et al., 1997). This was observed as an approximate $90 \%$ decrease in peak current between the first and second scans and a concurrent $9 \%$ decrease in current associated with the deposition of the nitrophenyl groups. As both trifluoromethylphenyl and nitrophenyl monolayer have been reported to prevent the oxidation of dopamine by glassy carbon electrodes (DuVal and McCreery, 1999) it was reasoned that the resulting surface would also share these properties while additionally providing nitrophenyl groups which could be reduced to aminophenyl groups, through the conversion of the nitro group to a nitroso, hydroxyl amine and finally amine through electrode catalyzed reduction (Allongue et al 1997), allowing for aptamer attachment sites (Figure 3.2).

To confirm that the heterogeneous trifluoromethylphenyl-aminophenyl monolayer retained its ability to prevent the oxidation of dopamine, the electrodes were compared to the signal produced by polished glassy carbon electrodes. The electrodes were also examined for their ability to reduce signals from neurochemicals known to interfere with dopamine sensing: norepinephrine (Figure 3.3, 3.4), L-3,4-dihydroxyphenylalanine (L-DOPA), serotonin and ascorbic acid (Gonon et al., 1980; Liu and Wang 1994). Additionally, the surface was examined for its ability to reduce electrochemical signal induced by the analogues of dopamine, 3,4-dihydroxyphenylacetic acid and tyramine. Tyramine and norepinephrine are known ligands of the dopamine aptamer (Walsh and DeRosa 2009). 

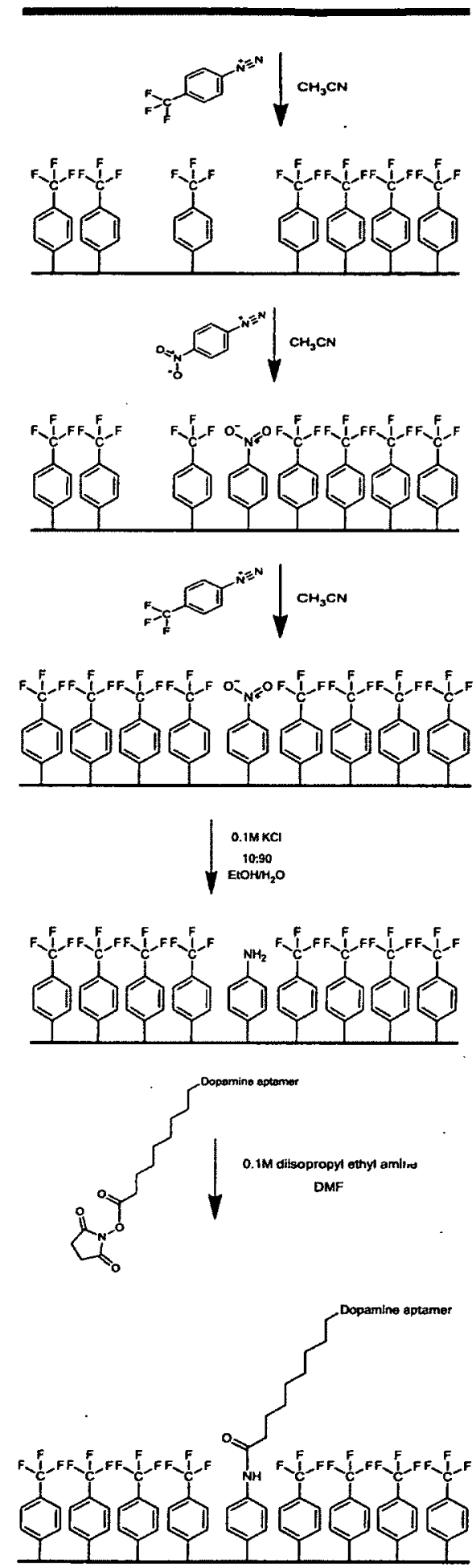

Figure 3.1. Reaction scheme for production of the dopamine aptamer functionalized glassy carbon electrode. 


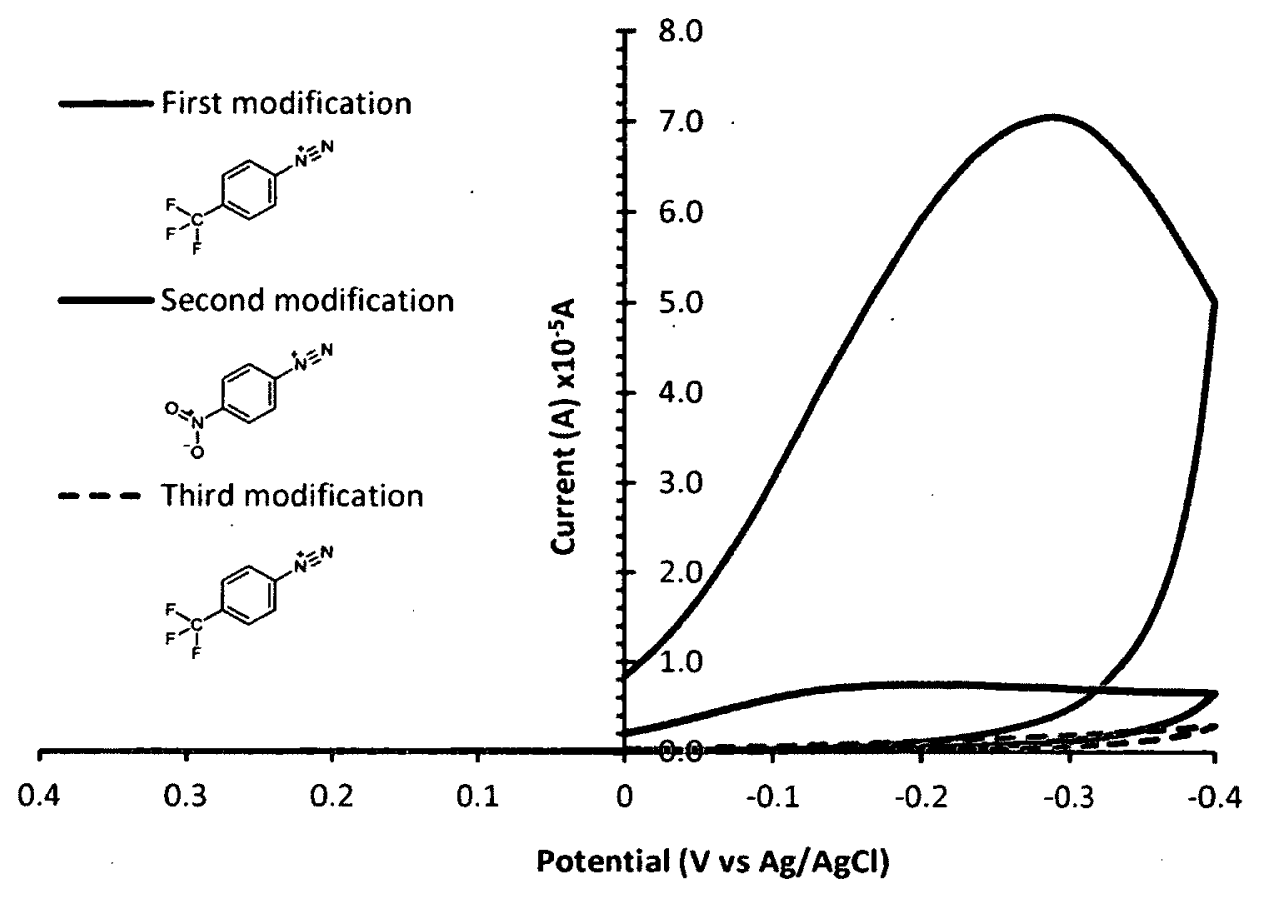

Figure 3.2. Glassy carbon electrode surface passivation through successive scans using $0.1 \mathrm{M}$ 4-(trifloromethyl)benzenediazonium in acetonitrile and $0.1 \mathrm{M}$ 4nitrobenzenediazonium in acetonitrile. This produced a heterogeneous trifluoromethylphenyl-nitrophenyl monolayer. 


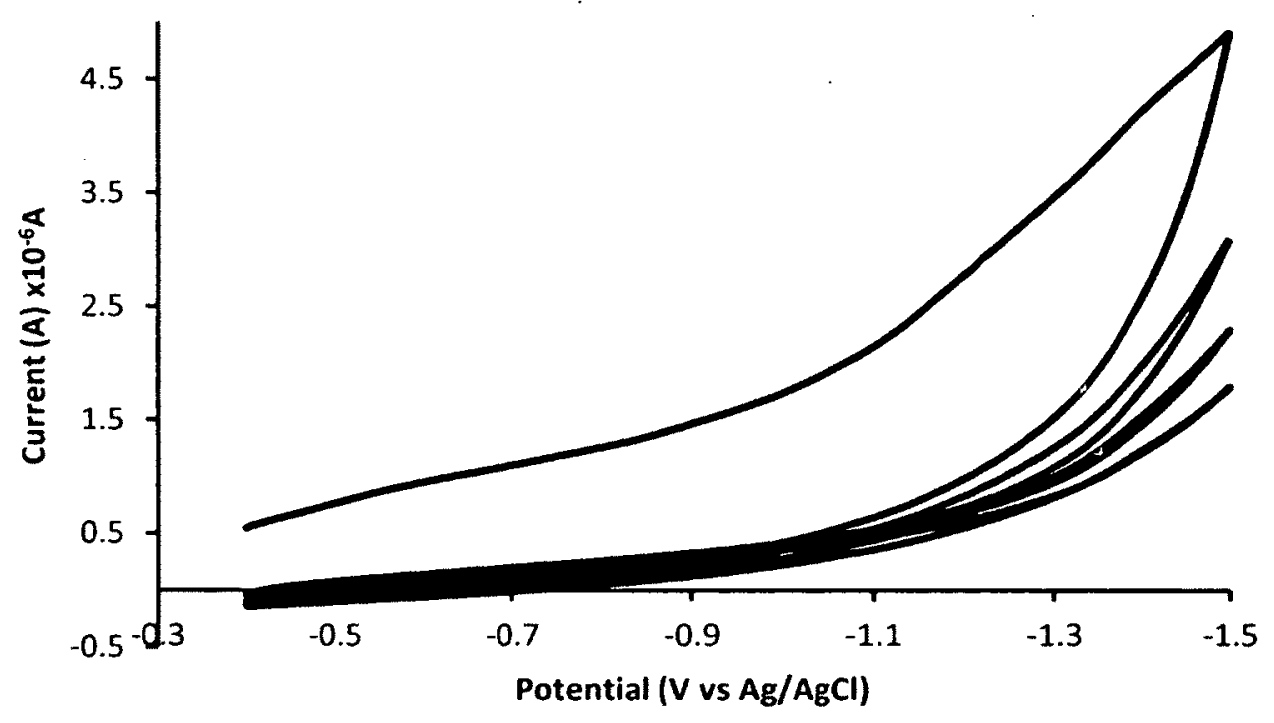

Figure 3.3. Nitro to amine conversion of surface nitro groups was accomplished using repetitive scans between -0.4 and $-1.5 \mathrm{~V}$ in $0.1 \mathrm{M} \mathrm{KCl} 10: 90$ ethanol and water. 


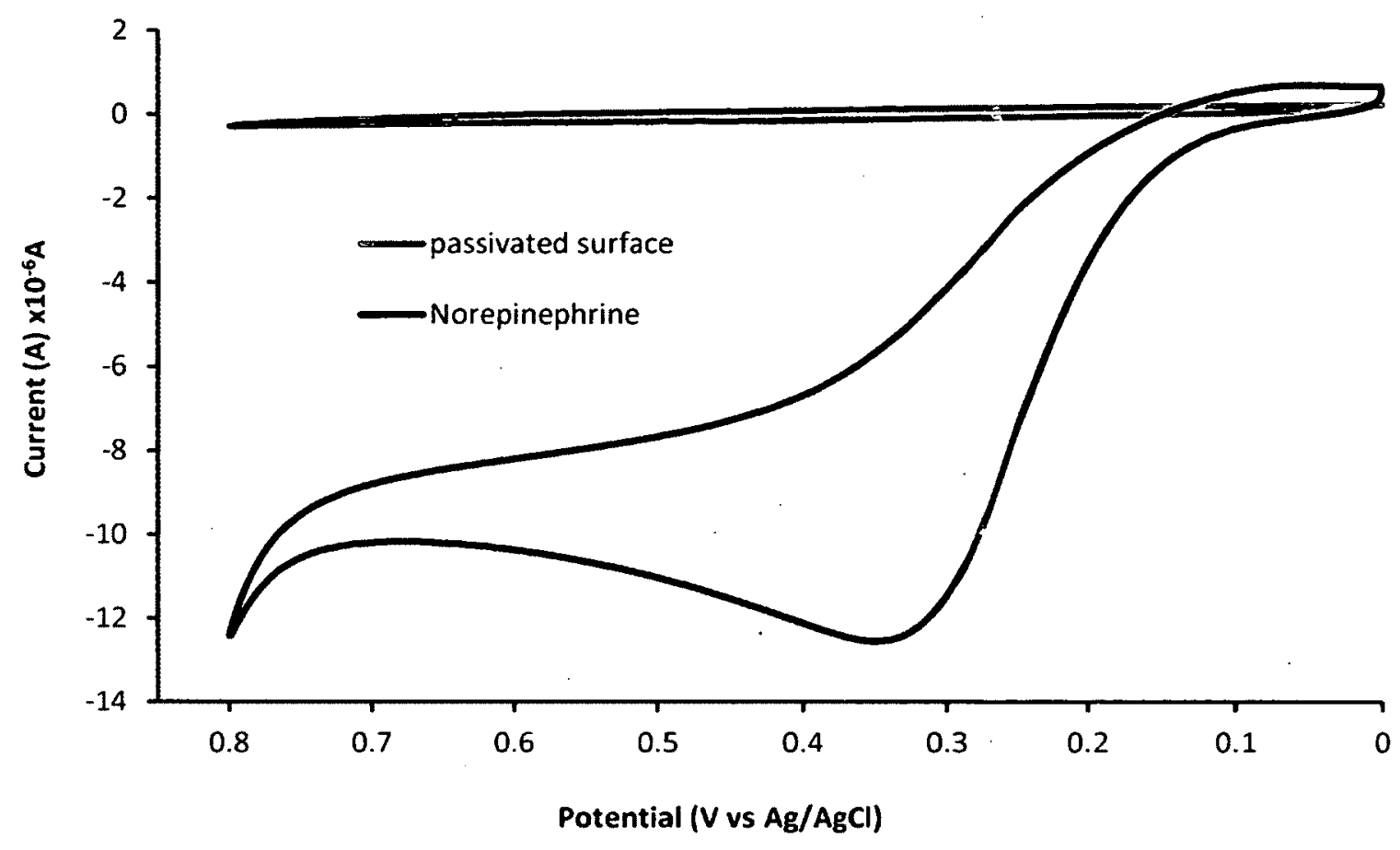

Figure 3.4. Cyclic voltammogram characterization $(0$ to $0.8 \mathrm{~V})$ of the passivation of a glassy carbon electrode. The response of the polished glassy carbon electrode to the catecholamine norepinephrine was compared to the signal prcduced after the surface passivation with the heterogeneous trifluoromethylphenyl-aminophenyl monolayer coating. 
Circular voltammetric and square wave characterization of the modified surface was consistent with previous reported surface passivation to catecholamine oxidation with trifluoromethylphenyl (DuVal and McCreery, 1999). The surface modification also cut off signals produced by ascorbic acid and serotonin (Figure 3.5).

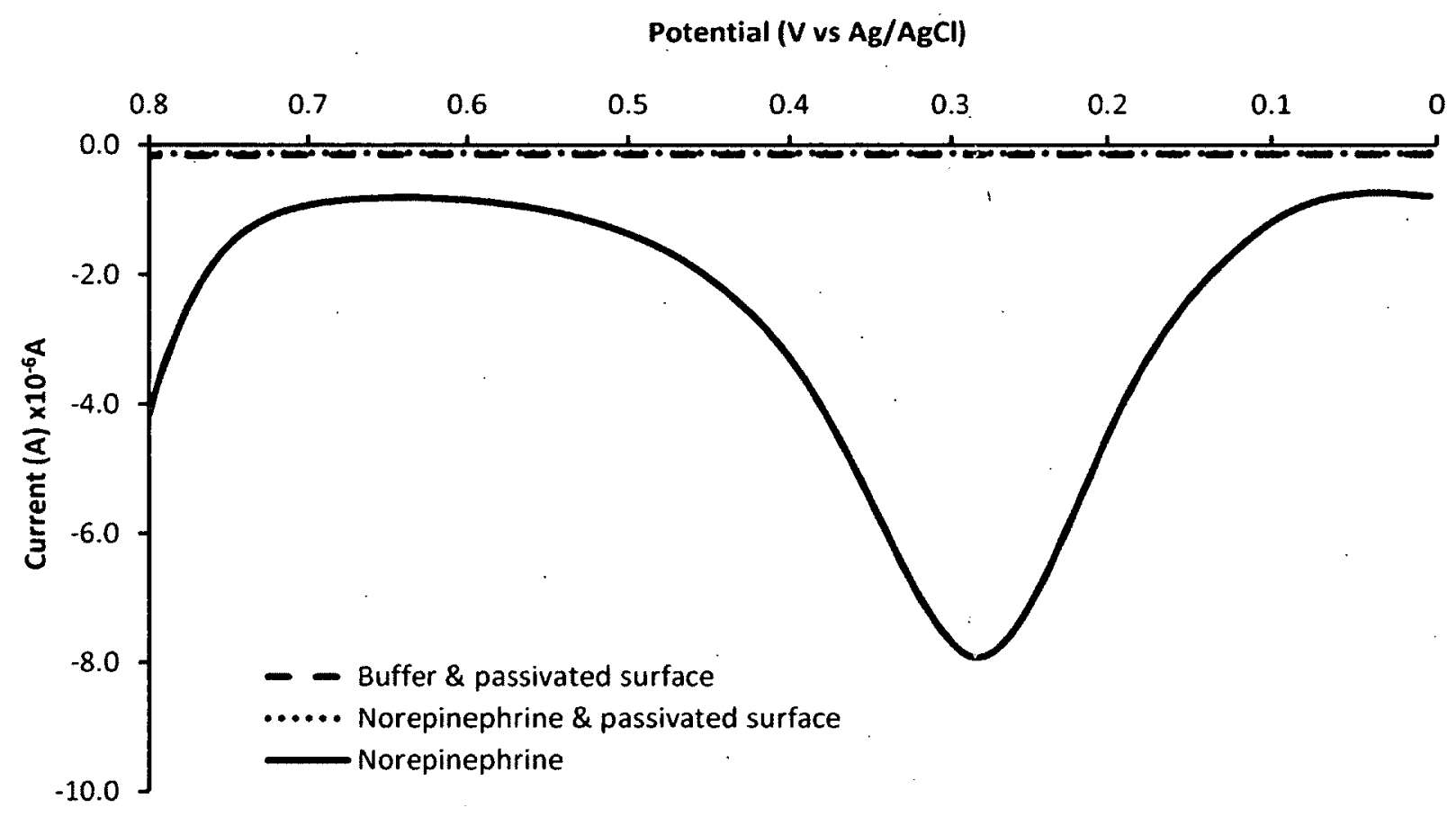

Figure 3.5. Square wave characterization $(0$ to $0.8 \mathrm{~V})$ of the passivation of a glassy carbon electrode. The response of the polished glassy carbon electrode was compared to the signal produced after the passivation of the surface with the heterogeneous trifluoromethylphenyl-aminophenyl monolayer coating. The observed signal after passivation was comparable to that of buffer alone. 
Covalent linkage of the aptamer to the passivated glassy carbon electrode allowed for the tuning of the surface to ligands specific to the aptamer (Table 3.1, Figure 3.6). As previously described, the DNA homologue of the dopamine aptamer is not entirely specific for dopamine (Walsh and DeRosa, 2009). However, based on the intensity of current observed in the presence of different catechol containing compounds, the surface functionalization did impact the ligand specificity of the aptamer. In solution, the aptamer was observed to have a slightly higher affinity for norepinephrine (Walsh and DeRosa 2009) while the ability of the surface functionalized aptamer to accommodate norepinephrine was reduced compared to the response observed with dopamine. In general, the aptamer functionalized electrodes had some sensitivity to all the examined catechols and analog compounds, with peak current potentials (170$200 \mathrm{mV}$ ) consistent with the oxidation of the catechols to their corresponding quinones at neutral pH values (Giacomelli et al., 2002). The specificity of the electrodes for dopamine however was quite apparent as the observed signals for the other compounds did not exceed $20 \%$ that observed with dopamine at equivalent concentrations of $1 \mathrm{mM}$ (Table1). The aptamer functionalized surface also produced a signal with serotonin and tyramine but both signals were observed to be well displaced from catecholamine signal (Table 3.1, Figure 3.6). Additionally, the electrode was completely unresponsive to ascorbic acid (Table 3.1) which may be attributed to the inclusion of ascorbic acid in the original SELEX selection buffer for the RNA dopamine aptamer (Mannironi et al., 1997). 


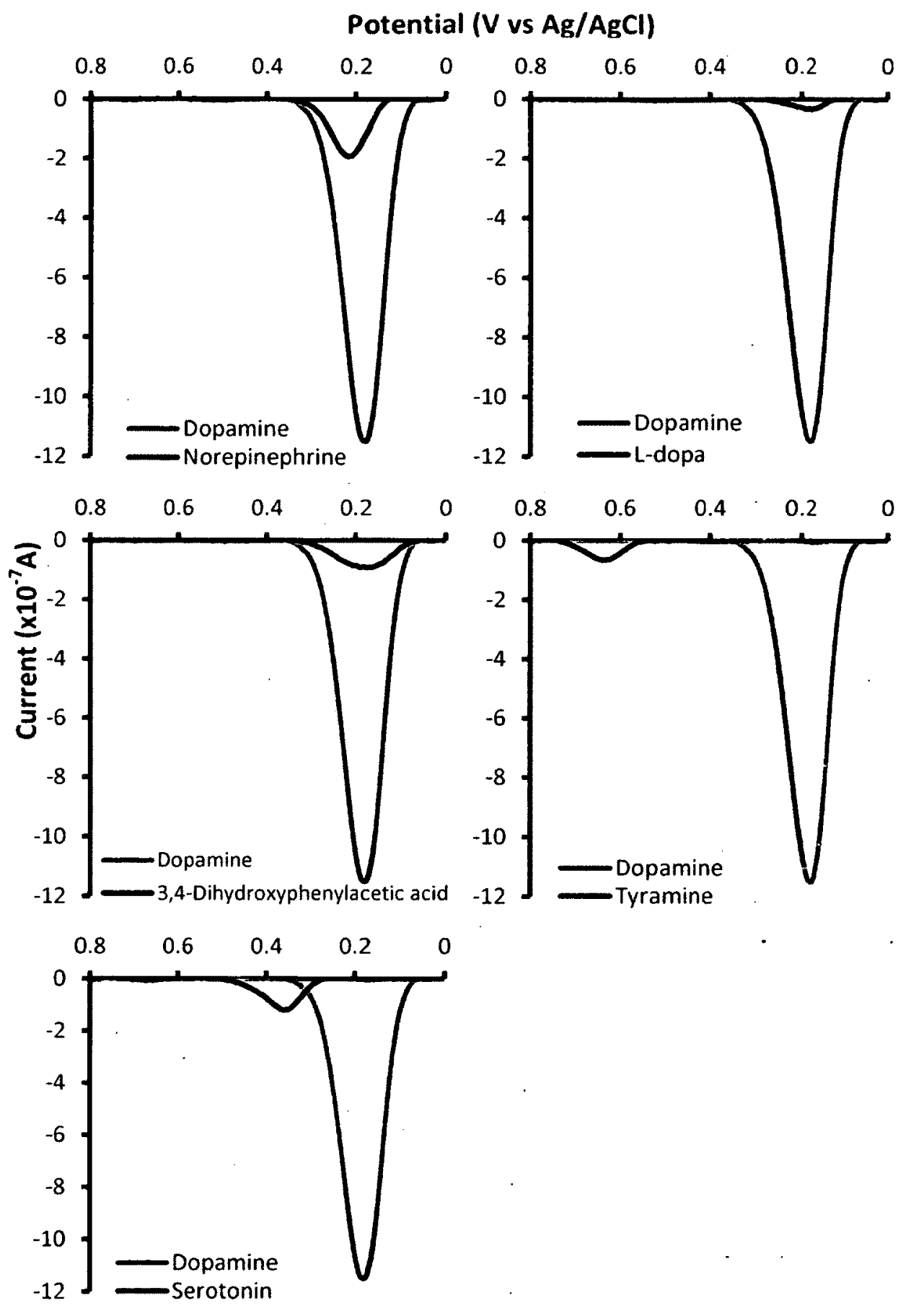

Figure 3.6. Square wave voltomic characterization $(0-0.8 \mathrm{~V})$ of the relative signals produced by the dopamine aptamer functionalized electrode with $1 \mathrm{mM}$ dopamine and A) $1 \mathrm{mM}$ norepinephrine, B) $1 \mathrm{mM}$ L-dopa, C) $1 \mathrm{mM}$ 3,4-Dihydroxyphenylacetic acid, D) $1 \mathrm{mM}$ tyramine, E) $1 \mathrm{mM}$ serotonin. 
An examination of the responsiveness of the electrodes to dopamine indicated that, while the aptamer provided selectivity to the surface, the functionalization of the aptamer to the electrode resulted in less signal than would be expected based solely on the dissociation constant attributed to the aptamer free in solution. While the dissociation constant for the aptamer in solution is in the tenth of micromolar range (Walsh and DeRosa 2009) the linear response range for the electrode was found to be between 27 and 1000 micromoles with a limit of detection of approximately $8 \mu \mathrm{M}$ (Figure 3.7). Compared to other electrodes in the literature, this places the sensitivity of the electrode on the higher side of the detection range for dopamine (Ahammad et al., 2011; Tian et al., 2012; Ping et al., 2012). Previously developed glassy carbon electrodes have possessed detection ranges for dopamine in the lower micromolar range ( 1-100 $\mu \mathrm{M})$, but most have omitted studies of other electrochemically active neurochemicals which might interfere with the dopamine signal, such as ascorbic acid (Ahammad et al., 2011; Tian et al., 2012). More recent studies using glassy carbon electrodes functionalized with tellurium-nanowires (Tsai et al., 2012) and functionalized graphene electrodes (Ping et al., 2012) have been able address interference by resolving the oxidative currents between dopamine and possible sources of interference with sensitivities in the nanomolar to low micromolar range. While in the higher range of sensitivity, the aptamer functionalized electrode represents a novel, selective method of dopamine detection relying on cutting off of interfering signals rather than by overpotential shifts. 

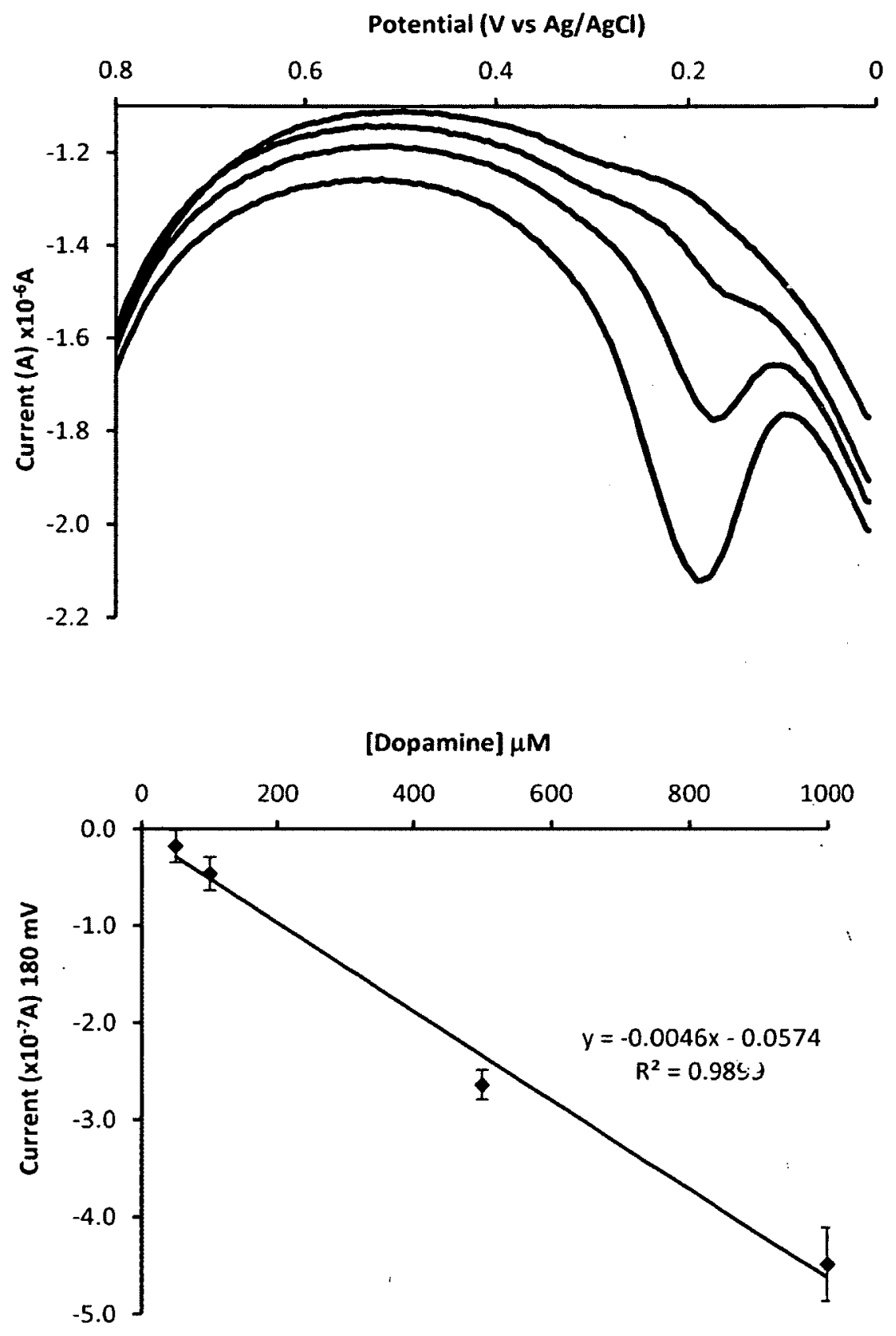

Figure 3.7. Assessment of the electrode detection range for dopamine using A) square wave scans from $0-0.8 \mathrm{~V}$ in increasing concentrations of Dopamine $(50,100,500 \&$ $1000 \mu \mathrm{M})$. B) Standard curve plot of peak current at $0.18 \mathrm{~V}$ versus Dopamine concentration. 
To confirm the electrodes signal was derived from specific interactions between the aptamer and dopamine the signal was compared to the signal produced with a random DNA sequence. The random DNA sequence was functionalized to a glassy carbon electrode using the same methodology as the aptamer and indicated that the signal resulted from the aptamer providing a specific interaction between the surface and the dopamine rather than a nonspecific signal caused. by interaction of the positively charged dopamine with the DNA backbone (Figure 3.8).

To further investigate the utility of the electrode for dopamine measurement, the electrodes were examined in the presence of dopamine mixed with ascorbic acid, norepinephrine and serotonin. The mixture of dopamine and ascorbic acid resulted in increased overpotential of the maximum current (Figure 3.9a). This could be attributed to the selection conditions used to generate the RNA dopamine aptamer which incorporated ascorbic acid (Mannironi et al., 1997) and may of increased the stability of the tertiary aptamer structure. Norepinephrine was observed to additively increase the current and also slightly increase the overpotential (Figure 3.9b). 


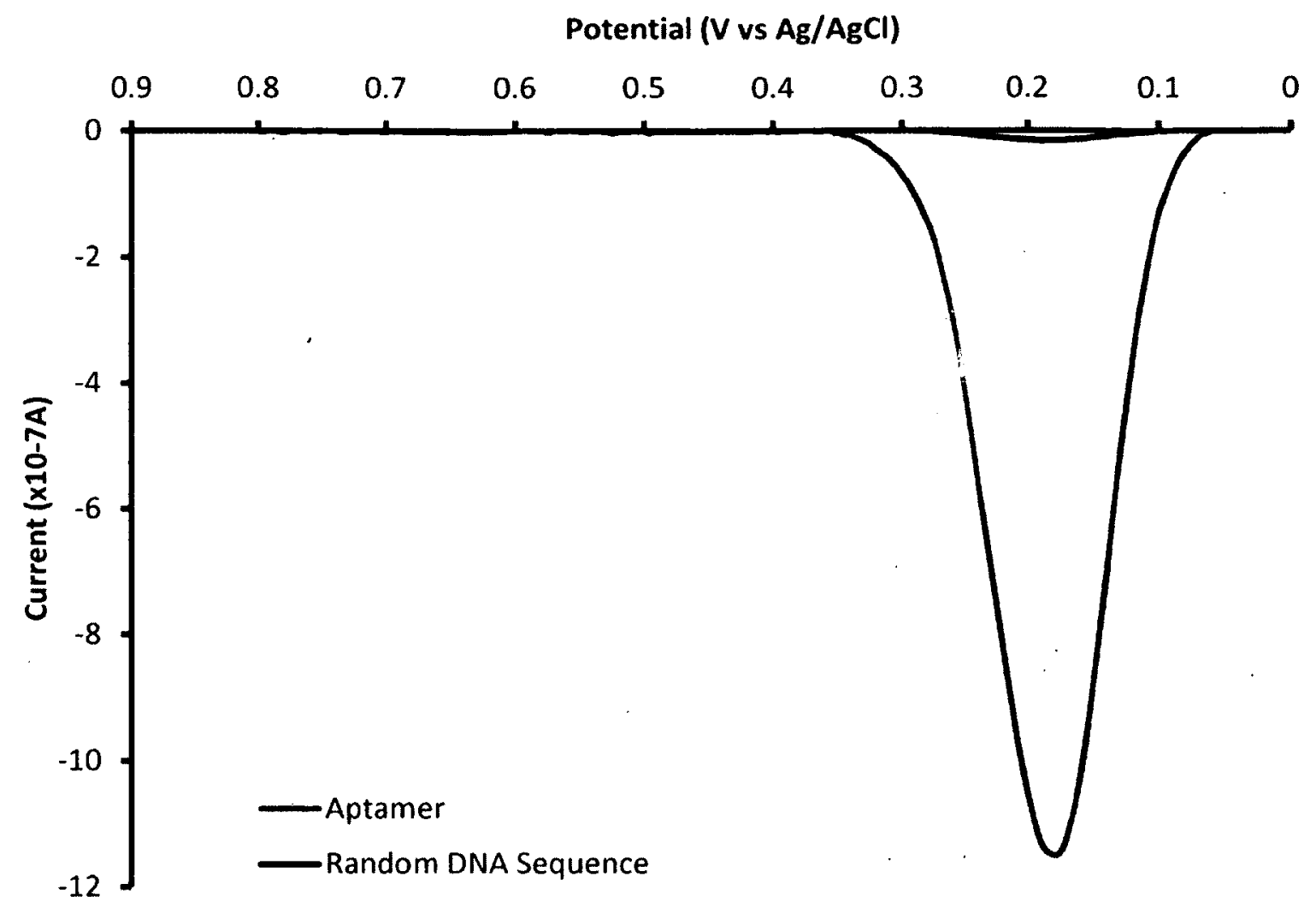

Figure 3.8. Square wave voltomic characterization $(0-0.8 \mathrm{~V})$ of the relative signals produced by the dopamine aptamer functionalized electrode with $1 \mathrm{mM}$ dopamine and a random DNA sequence functionalized electrode with $1 \mathrm{mM}$ dopamine. 

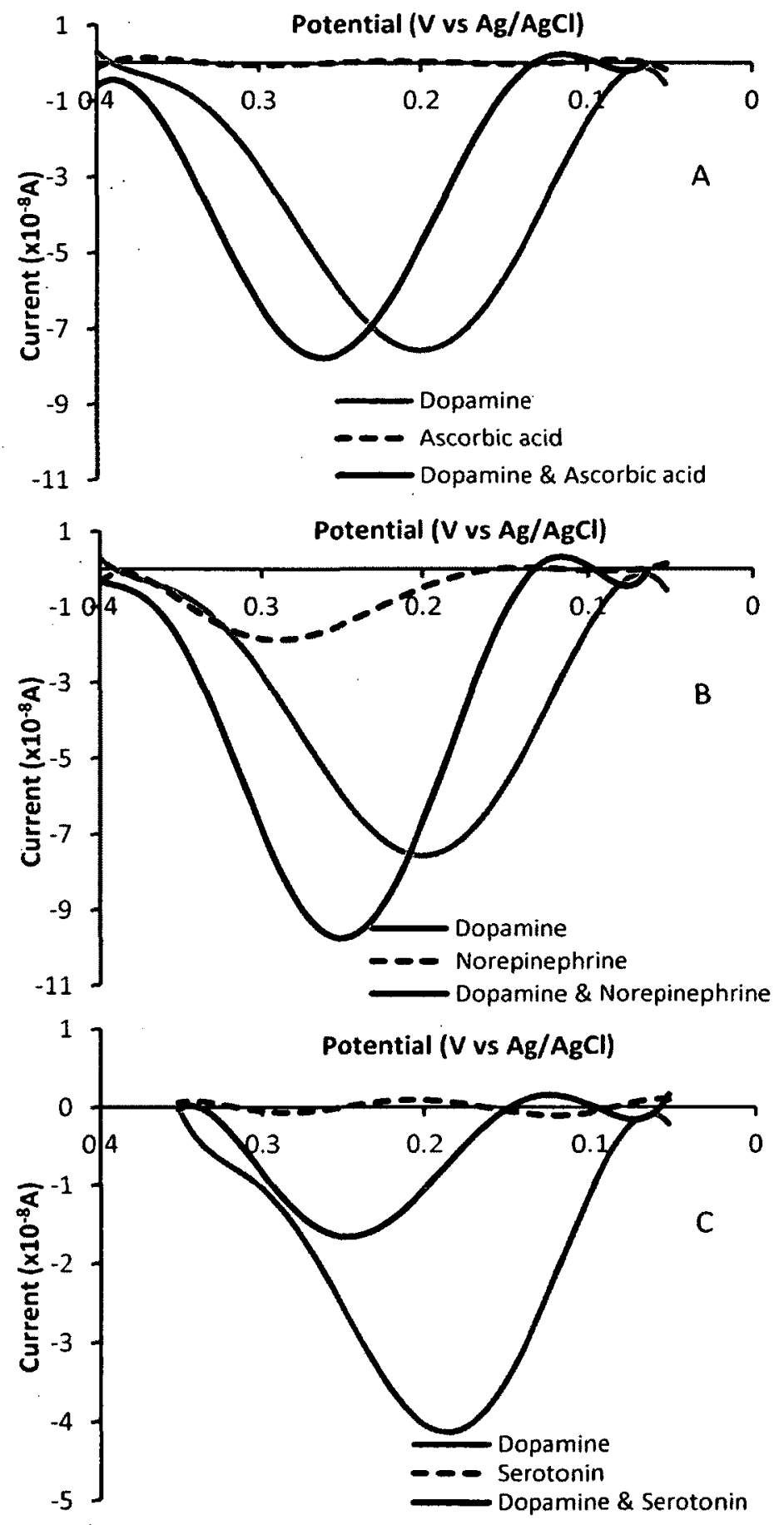

Figure 3.9. Square wave voltomic characterization of the relative signal affect of mixing $1 \mathrm{mM}$ dopamine with A) $1 \mathrm{mM}$ ascorbic acid, B) $1 \mathrm{mM}$ Norepinepherine and C) $1 \mathrm{mM}$ Serotonin. 
The observed increase in signal associated with norepinephrine may be attributed to the change in binding specificity observed with the free DNA aptamer (Walsh and DeRosa, 2009) such that the observed current resulted from an additive signal composed of both dopamine and norepinephrine. In contrast, mixing dopamine with serotonin decreased the dopamine peak and decreased the dopamine sensitivity with successive scans (Figure 3.9c). Fouling of the electrode surfaces is known to occur with serotonin as the oxidative species produced react with the surface and act as insulators (Swamy and Venton, 2007). While the signal generated by the aptamer functionalized electrode most likely does not result from direct surface interactions, reactive oxidative species produced by serotonin would potentially interfere with the structural integrity of the aptamer and eventually destroy the electrode. The in vitro electrode fouling produced using the $1 \mathrm{mM}$ concentration in this experiment was somewhat exaggerated as in vivo basal concentrations of serotonin are known to be in the nanomolar range (Crespi et al., 1988).

While specific signal interference can be expected from other catechol-containing compounds, one of the major sources of interference for dopamine detection arises from the presence of ascorbic acid (Gonon et al., 1980). Approaches to reduce ascorbic acid interference have focused on electrochemical pretreatment and monolayer depositions which increase the carbon to oxygen surface ratio, reducing the ability of ascorbic acid to interact with the surface while increasing the interaction of dopamine and catalyzing its oxidation (McCreery 2008). More recent work with fast scan cyclic voltammetry has also been able to reduce the interference of noncatechol containing 
compounds by providing voltammograms which allows for chemical identification (Zachek et al., 2010). We were able to address this problem by initially passivating the surface to all signals before re-establishing dopamine signalling through aptamer functionalization.

Efforts to reduce interference by other catechol containing compounds have also relied on surface modifications and the placement of electrodes in areas of the brain known to specifically express dopamine, reducing the potential for interference relating to the presence of other neurochemicals that electrochemically active in the range used to detect dopamine (Gonon et al.; 1980; Liu and Wang 1994; Zachek et al., 2010). Additionally efforts to confirm dopamine signals have required more sophisticated uses of sentential electrodes and pharmaceuticals to distinguish the neurochemical origins (Gonon et al., 1980; Zachek et al., 2010). We were able to demonstrate that the dopamine aptamer functionalized electrodes were able to address the problem of specific signal interference from other catechol containing compounds by exploiting a distinct preference for dopamine at equivalent concentrations.

\section{Conclusions and future perspectives}

The electrode functionalization for dopamine detection described here represents an efficient way for tuning glassy carbon electrodes for biosensor applications. The functionalization process based on passivation and the generation of selective binding surfaces using aptamer linked architectures is novel and deserves further investigation. 
The passivation process was performed in three steps with two separate diazonium solutions (Figure 3.2 ) suggesting that approximately $10 \%$ of the surface was modified with amine groups which could be later functionalized. The stepwise addition of the separate diazonium solutions resulted in the fixed coverage ratio, however, studies which address mixing of the two solutions may allow for optimization of the surface amine ratio for aptamer attachment.

Alternatively, the presence of aminophenyl groups in the organic monolayer may not be the ideal attachment system for the aptamer as the functionalization process requires highly basic and organic conditions, which are not conducive with DNA solubility. Bannwarth and Knorr (1991) described a system for forming carboxamides using $N, N, N^{\prime}, N^{\prime}$-tetramethyl (succinimido) uronium tetrafluoroborate in approximately $10 \% \mathrm{H}_{2} \mathrm{O}$ which may allow for improved DNA solubility and electrode functionalization. Improvements in DNA solubility could also be addressed by producing a carboxylphenyl monolayer that would be the target of the TSTU activation. This would allow the addition of an amine functionalized aptamer in a separate step where less extreme conditions would be required (Wilchek et al., 1994).

Additionally, other strategies for diazonium based functionalization of electrode with biological molecules have been described and may provide alternative routes for attaching the dopamine aptamer to the glassy carbon surface. Yang et al., (2005) have described a way of linking biological molecules by initially producing an aminophenyl monolayer and then attaching a spacer molecule (sulfo-succinimidyl 4-(N- 
maleimidomethyl)cyclohexan-1-carboxylate) to provide a place for thiolated DNA attachment. A similar methodology has been described by Harper et al., (2008) who also used a phenylmaleimide group as the electron acceptor in the attachment of thiolated DNA to the electrode surface although in this case the phenylmaleimide group arises directly from the $\mathrm{N}$-phenylmaleimide diazonium.

In general, the aptamer functionalized surface was able to provide a degree of selectivity for dopamine detection that is so far lacking in carbon electrode work. This study shows that aptamer functionalization of carbon electrodes in combination with electrode passivation has the potential to greatly improve the tuning of their selectivity towards biological targets.

\section{Acknowledgements}

I would like to thank Uyen Ho, Xiao Li Wang for their assistance in running some of the electrode scans. 
Table 3.1. Specificity characterization of the aptamer functionalized glass carbon electrode

\begin{tabular}{|lll}
\hline Lompound & Peak Current & Relative Current \\
(1mM)
\end{tabular}


Chapter 4 Systematic Evolution of Ligands by EXponential enrichment (SELEX) for DNA aptamers of Dopamine 


\begin{abstract}
In agreement with previous work, our demonstration that the binding characteristics of the RNA dopamine aptamer could be improved by simply converting it to its DNA homolog suggests that DNA is functionally comparable to RNA as a structural building block for aptamers. To examine this further, SELEX for DNA dopamine aptamers was undertaken using the same selection criteria used to produce the RNA aptamer for dopamine. The selection process for the DNA aptamers was performed in half the number of SELEX rounds and produced an aptamer which had an approximate 28 fold improvement in affinity for dopamine when compared to the RNA aptamer. The reduced selection period and the production of an aptamer of improved affinity can be attributed to an improvement of our selection protocol when compared to the RNA aptamer selection procedure rather than as an indication that DNA is functionally superior as an aptamer selection material.
\end{abstract}




\section{Introduction}

The ability to quantify dopamine is of particular importance as it is known to play a major role in fine motor control and the formation of addictive behaviour (Klein et al., 2007). Dopaminergic dysfunction has been linked to Schizophrenia (Basile etal., 2002) and the death of dopaminergic neurons in the substantia nigra results in the tremor motion or motor impairment associated with Parkinsonism (Lees et al., 2009). The ability to quantity normal and abnormal dopaminergic activity may potentially shed a great deal of insight into these processes and may be particularly addressable with aptameric techniques.

As shown in chapter 3, the DNA homolog of the RNA dopamine aptamer (Walsh and DeRosa, 2009) could be used to functionalize electrodes, improving their specificity to dopamine over that of other catecholamine containing compounds. However, the selectivity of the electrode for dopamine was accompanied by a somewhat high detection limit. Whether this could be addressed by adjusting the electrode functionalization conditions or if the propertied of the aptamer were actually altered as a result of its sequestering on the surface is a matter for further investigation. However, the conversion of the RNA dopamine aptamer (Mannironi et al., 1997) to a functional DNA homolog (Walsh and DeRosa, 2009) demonstrated the structural similarities between DNA and RNA and suggest that a DNA based Systematic Evolution of Ligands by EXponential enrichment (SELEX) selection for dopamine may allow for the production of aptamers more suited for this application. 
SELEX is a dynamic process that has allowed for the production of aptamers of specific selectivity and high affinity for a variety of targets both large and small. SELEX has been used to select for binders to cell for such applications as cancer detection (Graham and Zarbl 2012), proteins for applications such as the diagnosis of Alzheimer's disease (Rahimi and Bitan, 2010) and small molecules like ATP (Huizenga and Szostak, 1995) which may provide novel ways of monitoring cellular metabolism. The selection of aptamers ideally should start with maximal diversity in the DNA or RNA pools, to do this pools are usually generated with random regions of sequence around 20 to 30 bases in length, although some selections have involved random sections as large as 80 nucleotides (Stoltenburg et al., 2007). Pools with larger random sections may provide more potential diversity for generating aptamers, huwever due to the exponential relationship between length of the random sequence and increased diversity of individual sequences in the pool, random sequences around 20 nucleotides in length provide about equal diversity to the actual number of molecules used to initiate a selection. The shorter length of these starting pools may be the limiting factor in the somewhat simple structural profile of most aptamers, such as stem loops and Gquadruplexes, however methods such as computer-aided pool design have been developed to increase structural diversity with the goal of producing better aptamer sequences (Luo et al., 2010).

The utility of the aptamer produced by a selection process is dependent on the selection conditions and careful consideration must be given to the final application as the structural stability of the aptamer may be affected by conditions such as ionic 
strength, $\mathrm{pH}$ and temperature (Cho et al., 2009). Other considerations, particularly when dealing with small molecules, involve the method of functionalizing the target molecule as chemical modifications may drastically change the binding specificity of isolated aptamer sequences. This may partially explain relatively high affinity constants $(\mu \mathrm{M})$ associated with aptamers for small molecules compared to the nanomolar to picomolar affinities reported for larger molecules like proteins and viruses (Stoltenburg et al., 2007) . For example, the aptamer for ATP has been reported as $6 \mu M$ (Huizenga and Szostak, 1995), while the aptamer for keratinocyte growth factor, a protein involved in wound healing, has been reported as low as 1 pM (Pagratis et al., 1997).

Here, we further examine the ability of DNA to replicate the functional capabilities associated with the RNA dopamine aptamer by examining the selection process used to produce the RNA Dopamine aptamer (Mannironi et al., 1997) and applying a similar SELEX methodology in the production of DNA aptamers for dopamine.

\section{Materials and methods:}

Acrylamide, dimethylformamide, dopamine hydrochloride, formamide, N,N'methylenebisacrylamide, and $N, N, N^{\prime}, N^{\prime}$-tetramethylethylenediamine were purchased from Sigma-Aldrich. 5-Bromo-4-chloro-3-indoyl- $\beta$-D-galactopyranoside (X-gal), LB broth, dNTP mix, and Taq DNA polymerase were purchased from Bioshop. LB-Agar Ampicillin plates were acquired from Health Canada. The Dopamine-Agarose column was 
purchàsed from MP Biomedicals. StrataClone ${ }^{r M}$ PCR Cloning 'it was purchased from Stratagene.

Primer 1: 5'-ATACCAGCTTATTCAATT-3'

Primer 2: 5'-AGATTGCACTTACTATCT-3'

Primer 1 with Fluorescein tag: 5'-RATACCAGCTTATTCAATT-3'

Primer 2 with Poly $A_{20}$ tail and ethyleneglycol spacer:

5'-AAAAAAAAAAAAAAAAAAAAXAGATTGCACTTACTATCT-3'

DNA Pool sequence $\mathbf{N}_{60}$ random sequence:

$5^{\prime}-$ ATACCAGCTTATTCAATTNNNNNNNNNNNNNNNNNNNNNNNNNNNNNNNNNNNNNNNN NNNNNNNNNNNNNNNNNNNNAGATAGTAAGTGCAATCT-3'

The above sequences were initially used by Stoltenburg et al. (2005), where R represents the Fluorescein label 6-Fluorescein Phosphoramidite, $\mathbf{X}$ represents Spacer Phosphoramidite 18, the hexaethyleneglycol spacer which prevents TAQ polymerase read through, and $\mathbf{N}$ represents a random base in the sequence which could be adenine, thymine, cytosine or guanine. 


\section{DNA based selection for dopamine aptamers}

The DNA pool was suspended in $1 \mathrm{~mL}$ of selection buffer, $50 \mathrm{mM}$ Tris- $\mathrm{HCl} \mathrm{pH}$ 7.4, $5 \mathrm{mM} \mathrm{MgCl} 2,0.5 \mathrm{M} \mathrm{NaCl}, 0.02 \% \mathrm{~L}$-ascorbic acid and heated to $90^{\circ} \mathrm{C}$ for 10 minutes before being allowed to cool to room temperature. The pool was then introduced into a counted selection agarose column which was prepared in a microfilterfuge tube $(0.45$ $\mu \mathrm{m}$ nylon filters) preequilibrated with $50 x$ the column volume selection buffer. The pool was mixed for 5 minutes before being collected for addition to the $1 \mathrm{~mL}$ dopamineagarose column. The pool was allowed to equilibrate with the column for an hour and then washed with $1 \mathrm{~mL}$ volumes of selection buffer and monitored with fluorescence to ensure removal of nonbinding sequences. Dopamine binding sequences were eluted off the column with $0.1 \mathrm{M}$ dopamine suspended in the selection buffer. The eluted samples were initially concentrated then desalted with using 3,000 NMIWL Millipore Microcon centrifugal filter devices $12000 \mathrm{~g}$ for 30 minutes, before being dried down for PCR amplification and isolation (See sections below).

The second round of selection followed the same procedure as the first except the isolate pool did not travel through an initial agarose counter selection column and the Dopamine selection column was reduced in volume to $200 \mu \mathrm{L}$. .

Round three of SELEX was performed in the same way as round two and round 4 also followed the same protocol. Using the $0.1 \mathrm{M}$ dopamine elution technique on round 4 of the selection failed to elute significant samples which could be amplified with PCR and was attributed to a loss of specific column binding. To recover viable aptamer 
sequences the column from round 3 was rewashed with $7.4 \mathrm{M}$ urea suspended in selection buffer at $90^{\circ} \mathrm{C}$. The isolated sequences from round 4 were recombined with the Urea eluted sequences and subjected to a finai round of selection where the sequences were eluted from the column with $7.4 \mathrm{M}$ urea suspended in selection buffer at $90^{\circ} \mathrm{C}$. The eluted samples were desalted and dried down for PCR amplification and isolation of double stranded DNA for cloning.

\section{PCR}

Samples to be amplified were suspended in reaction buffer consisting of $100 \mathrm{mM}$ Tris $\mathrm{HCl} \mathrm{pH} \mathrm{9,} 50 \mathrm{mM} \mathrm{KCl}, 1 \%$ Triton X-100, $2 \mathrm{mM} \mathrm{MgCl} 2,0.5 \mathrm{mM}$ dNTPs, $1 \mu \mathrm{M}$ of each primer and $5 \mathrm{U}$ of Taq DNA polymerase in a final volume $100 \mu \mathrm{L}$. Samples were amplified using an Eppendorf Mastercycler EP Gradient, Thermal Cycler programmed to initially denature samples at $95^{\circ} \mathrm{C}$ for 2 minutes followed by 30 rounds of amplification. Amplification cycles consisted of primer annealing at $57^{\circ} \mathrm{C}$ for 1 minute, followed by 1 minute of template amplification $72^{\circ} \mathrm{C}$ and concluding with sample denaturation for 30 seconds at $95^{\circ} \mathrm{C}$ before initiation of the following amplification cycle. The amplification process concluded with an extended 5 minute $72^{\circ} \mathrm{C}$ template amplification phase before samples were cooled to $4^{\circ} \mathrm{C}$ before processing. Amplified samples were dried down using a Savant DNA120 SpeedVac Concentrator, Thermo Electron Corporation, in preparation for electrophoretic isolation. 


\section{Polyacrylamide Gel Electrophoresis}

Samples were isolated with $12 \%$ acrylamide gels cast using $18 \times 16 \mathrm{~cm}$ gel plates. Twelve percent acrylamide gels were cast by combining $31.5 \mathrm{~g}$ of urea, $23.5 \mathrm{mLs}$ of 5.3 $\mathrm{M}$ acrylamide and $130 \mathrm{mM} \mathrm{N}, \mathrm{N}^{\prime}$-methylenebisacrylamide, $15 \mathrm{mLs}$ of $0.44 \mathrm{M}$ Boric acid, $0.44 \mathrm{M}$ Tris, and $10 \mathrm{mM}$ EDTA with $14 \mathrm{mLs} \mathrm{dH}_{2} \mathrm{O}$. The mixture was heated to $37^{\circ} \mathrm{C}$ filtered and allowed to cool to room temperature. This was followed by the addition of $0.45 \mathrm{~mL}$ of $10 \%$ ammonium persulfate followed by $35 \mu \mathrm{L} N, N^{\prime} N^{\prime}, N^{\prime}-$ tetramethylethylenediamine. The gels were poured and allowed to polymerize for 20 minutes. After polymerization, the gels were set up in a Hoefer SE 600 series vertical slab gel electrophoresis unit and the buffer reservoirs were filled with $88 \mathrm{mM}$ Boric acid, $88 \mathrm{mM}$ Tris, and $2 \mathrm{mM}$ EDTA. Gels were pre-equilibrated, typically using a constant current of 35 milliamps for 20 mins using a FisherBiotech Power Suppy FB1000. DNA samples were dissolved in minimal amounts of $\mathrm{dH}_{2} \mathrm{O}$ and mixed with an equal amount of formamide before being heated at $55^{\circ} \mathrm{C}$ for 5 minutes. This was followed by loading onto the gel for separation. The gels were subjected typically to a constant current of 35 milliamps for 2 hours.

Upon completion of the electrophoretic run, the gels were imaged using an Alpha Innotech imager. Bands of interest were cut out and the gel crushed and suspended in 5 times the volume of $10 \mathrm{mM}$ Tris $\mathrm{pH}$ 7.4. Samples were frozen using liquid nitrogen followed by heating to $90^{\circ} \mathrm{C}$ for 10 minutes. The samples were then allowed to mix and incubated overnight at $37^{\circ} \mathrm{C}$. Following incubation, the samples were filtered $(0.45 \mu \mathrm{m})$ to remove the remaining gel fragments. Samples were then lyophilized to reduce the 
sample volume followed by resuspension in a minimal volume of deionized water for desalting and concentration. Samples were concentrated initially followed by 4 rounds of deionization with deionized water using 3,000 NMWL Millipore Microcon centrifugal filter devices $12000 \mathrm{~g}$ for 30 minutes. Samples were then quantified by assessing their absorbance at $260 \mathrm{~nm}$ and lyophilized.

\section{Cloning and Sequencing}

Sequences of interest were cloned using the StrataClone PCR cloning kit. Double stranded DNA samples isolated from the gel were desalted, dried and resuspended in 50 $\mu \mathrm{L}$ and the concentration checked to assure it fell within the range of 5-50 ng/ $\mu \mathrm{L}$. The sample and reaction components were kept on ice prior to the combination of $2 \mu \mathrm{L}$ of the sample and $3 \mu \mathrm{L}$ of the provided StrataClone Cloning Buffer. This was followed by the addition of $1 \mu \mathrm{L}$ of the StrataClone Vector Mix amp. The sample was then mixed with the pipette and allowed to react for 5 minutes at room temperature. The reaction mixture containing the recombinant plasmids was then placed on ice $\left(4^{\circ} \mathrm{C}\right)$ again prior to cell transformation. One tube of StrataClone Solopack competent cells was thawed at $4^{\circ} \mathrm{C}$ prior to the addition of $1 \mu \mathrm{L}$ of the reaction mixture. The sample was gently mixed through inversion before incubation on ice for 20 minutes. The sample was then subjected to heat shock by incubating it at $42^{\circ} \mathrm{C}$ for 5 minutes followed by a further 2 minutes on ice. This was followed by the addition of $250 \mu \mathrm{L}$ of $L B$ medium at $42^{\circ} \mathrm{C}$ and incubation of the mixture at $37^{\circ} \mathrm{C}$ with continual mixing for an hour. A LB-ampicillin 
plate ( $1 \% \mathrm{NaCl}, 1 \%$ tryptone, $0.5 \%$ yeast extract, $2 \%$ agar and $100 \mu \mathrm{g} / \mathrm{mL}$ ampicillin) was prepared for cell culturing with the addition of $40 \mu \mathrm{L}$ of $2 \% \mathrm{X}$-gal in dimethylformamide. Once the plates were prepared, $50 \mu \mathrm{L}$ of the transformed cell suspension was plated onto the plates and the plates were incubated overnight at $37^{\circ} \mathrm{C}$. Plates were sent to Molecular Cloning Laboratories for sequencing of potential aptamer sequences.

\section{Anisotropy studies}

Anisotropy studies using dopamine as the reporter molecule were performed by exposing the dopamine to increasing concentrations of the potential aptamer sequences. All samples were prepared in $50 \mathrm{mM}$ Tris $\mathrm{HCl}(\mathrm{pH} 7.4), 5 \mathrm{mM} \mathrm{MgCl}, 0.5 \mathrm{M}$ $\mathrm{NaCl}$ with the concentration of dopamine being held constant as the aptamers concentrations was increased. Fluorescent data was collected with a $40 \mu \mathrm{L}$ fluorescent cell with a Fluorolog-3 Spectrofluorometer from HORIBA Jobin Yvon (Ex/Em $=285 / 317$ $\mathrm{nm}$ ). Binding affinity between the isolated sequences were determined as described in chapter 2.

\section{Results and discussion}

The selection of a DNA aptamer for dopamine was based on a combination of the protocol originally described for the selection of the RNA dopamine aptamer (Mannironi, et al., 1997) and the Flumag protocols developed by Stoltenburg et al., 
(2005) for non radioactive SELEX. The fluorescein labelling of the primer used in the pool amplification allowed for detection of potential aptamer sequences as they were passed through the selection columns as well as detection in the gel after electrophoresis. The competitive dopamine elution method, used by Mannironi et al., (1997), was initially used to elute sequences binding to the dopamine column (Figure 4.1). However analysis of the DNA being eluted from the selection column suggested that only a small fraction of the sequences which were passing through the selection columns were being eluted with the competitive dopamine elution method. This suggested the possibility that the $0.1 \mathrm{M}$ dopamine elution solution was not sufficient to competitively elute all the sequenced binding to the selection column. Therefore, the column material used in round three was re-examined using an elution solution containing $7.4 \mathrm{M}$ urea in selection buffer $90^{\circ} \mathrm{C}$, to disrupt hydrogen bonding involved in the binding and secondary of the DNA sequences (Priyakumar et al., 2009).

This revealed that the $0.1 \mathrm{M}$ dopamine solution had not been sufficient to elute all the sequences which had bound to the columns. After combining the newly isolated sequences with the sequences from round four, an additional round of SELEX was undertaken using the urea elution method. Due to the high degree of affinity between the pool and dopamine functionalized matrix, inferred by the need to use hot urea, the sequences eluted in this round were isolated and sequenced so that binding studies could be undertaken. 


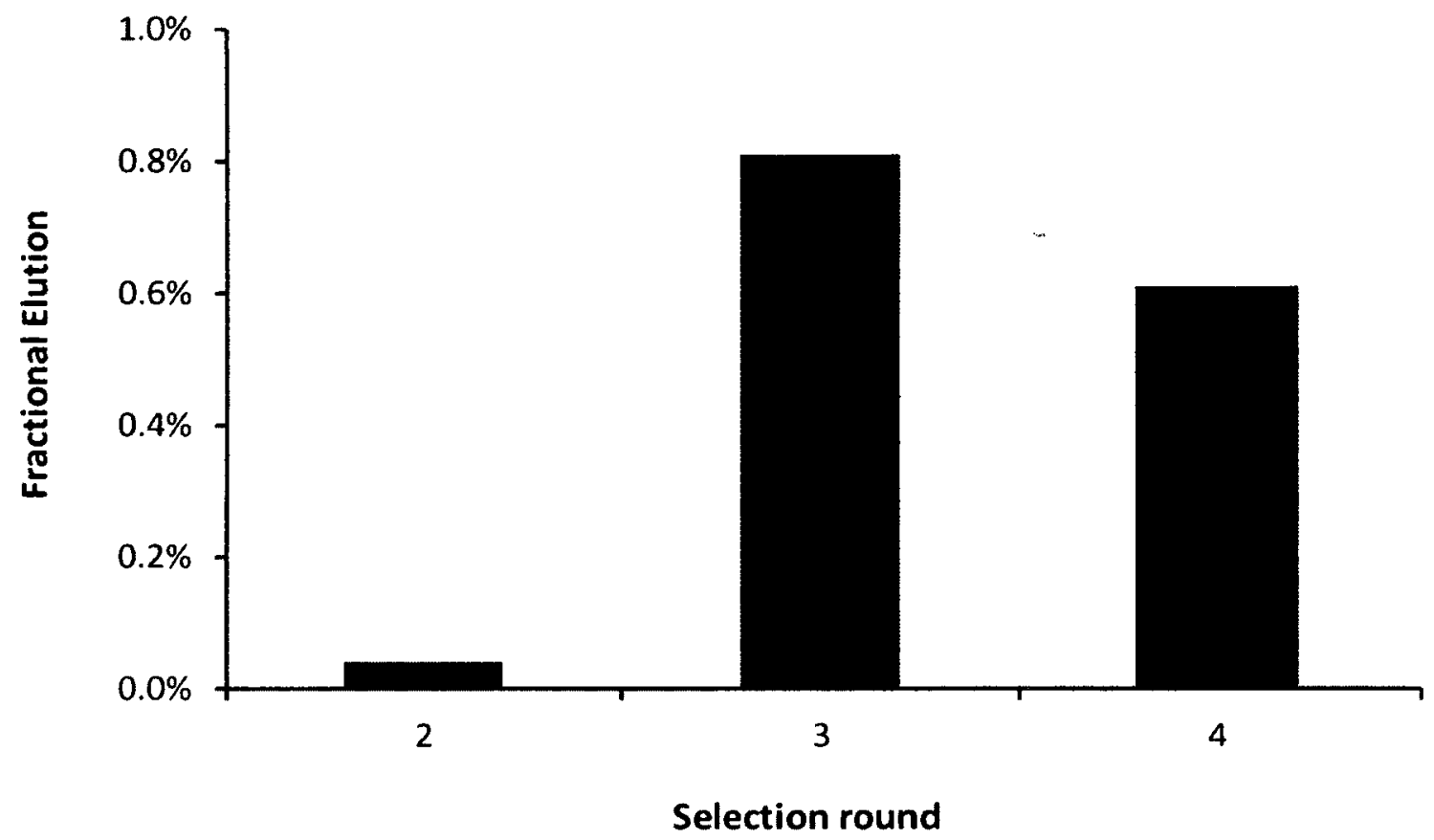

Figure 4.1. Pool sequences were eluted from the dopamine selection column competitively using $0.1 \mathrm{M}$ dopamine. The bars represent the approximate percent of the original pool which was eluted from the dopamine column during the second third and fourth selection rounds.

Sequencing resulted in the discovery that several of the sequences were more common than others and a BLAST analysis (Johnson et al., 2008) of the recovered sequences did not suggest any similarities between any of the individual isolated sequences. No similarities were found when the DNA sequences were compared to the DNA homolog of the RNA dopamine aptamer as well. Submission of the sequences to the Vienna RNA Websuite (Gruber et al., 2008) supported the BLAST results suggesting very little commonality in potential secondary structures (Table 4.1). These 
observations suggest that evolutionary processes were probably not a major contributor to aptamer generation in this selection, potentially due to the low number of selection rounds. This was further supported when the frequency of sequences was compared to their binding affinity for dopamine. Anisotropy studies indicated a range of binding affinities between 0.1 to $42 \mu \mathrm{M}$ (Table 4.1). However, surprisingly, the most prevalent sequences represented the extremes of that range with both DopaA20 ( $\left.K_{d} 0.107 \mu M\right)$ and DopaB3 (Kd $42.4 \mu \mathrm{M})$ appearing three times. The remaining sequences appeared one or two times and fell between 3 and $34 \mu \mathrm{M}$. This is fairly similar to the range of affinities published for most small molecules in the literature, for example the aptamer for arginine has been reported as $0.3 \mu \mathrm{M}$ (Geiger et al., 1995) and the aptamer for citrulline has been reported around $64 \mu \mathrm{M}$ (Famulok M. 1994).

The range of binding affinities produced by the DNA SELEX selection for dopamine is quite interesting as the RNA dopamine aptamer selection occurred over twice the number of selection rounds but only produced an aptamer with an affinity of $2.8 \mu \mathrm{M}$. Although, when a minimal structure analysis was applied, the affinity was improved to $1.6 \mu \mathrm{M}$ (Mannironi et al., 1997). While a dissociation constant of $2.8 \mu \mathrm{M}$ does fall into the higher affinity sequences observed in this study, it is more than 28 fold worse than the best binding affinity observed after only four rounds of selection in this study. This suggests that the competitive elution used in the selection of the RNA aptamer was not optimal and may have left many of the best binding sequences on the selection column, although, under certain conditions competitive elution may be able to improve the selectivity of aptamer sequences. 
The sequences produced in this selection for a dopamine aptamer represent a wide range of affinities which may prove useful in the detection of dopamine. Typical binding isotherms only cover a target responsiveness of three orders of magnitude centered around their dissociation constant. Although receptor clustering may be able to improve the lower limits (Caré and Soula, 2011) a sensor constructed using multiple receptors with different affinities for the target may produce a larger response range. This may be of practical importance as neuronal dopamine levels have been observed in the millimolar concentration range (Lent et al., 1983). While ultimately the DNA aptamers produced in this selection may be utilized in sensing applications, additional studies to assess their specificity for dopamine relative to other catecholamines still needs to be assessed. Additionally, as the binding properties of almost all aptamers invariantly change with modification (Cho et al., 2009) characterization of specificity and responsiveness upon attachment to electrodes also needs to be evaluated.

\section{Acknowledgements}

I would like to thank Erin McConnell for her work on the selection of these aptamers and characterization of their binding. 
Table 4.1. SELEX identified aptamer sequences with potential secondary structures predicted using the RNAfold suit of the Vienna RNA Websuite (Gruber et al., 2008) and experimentally determined dopamine binding constants. The bracketed number under their name indicates the prevalence of each sequence during sequencing.

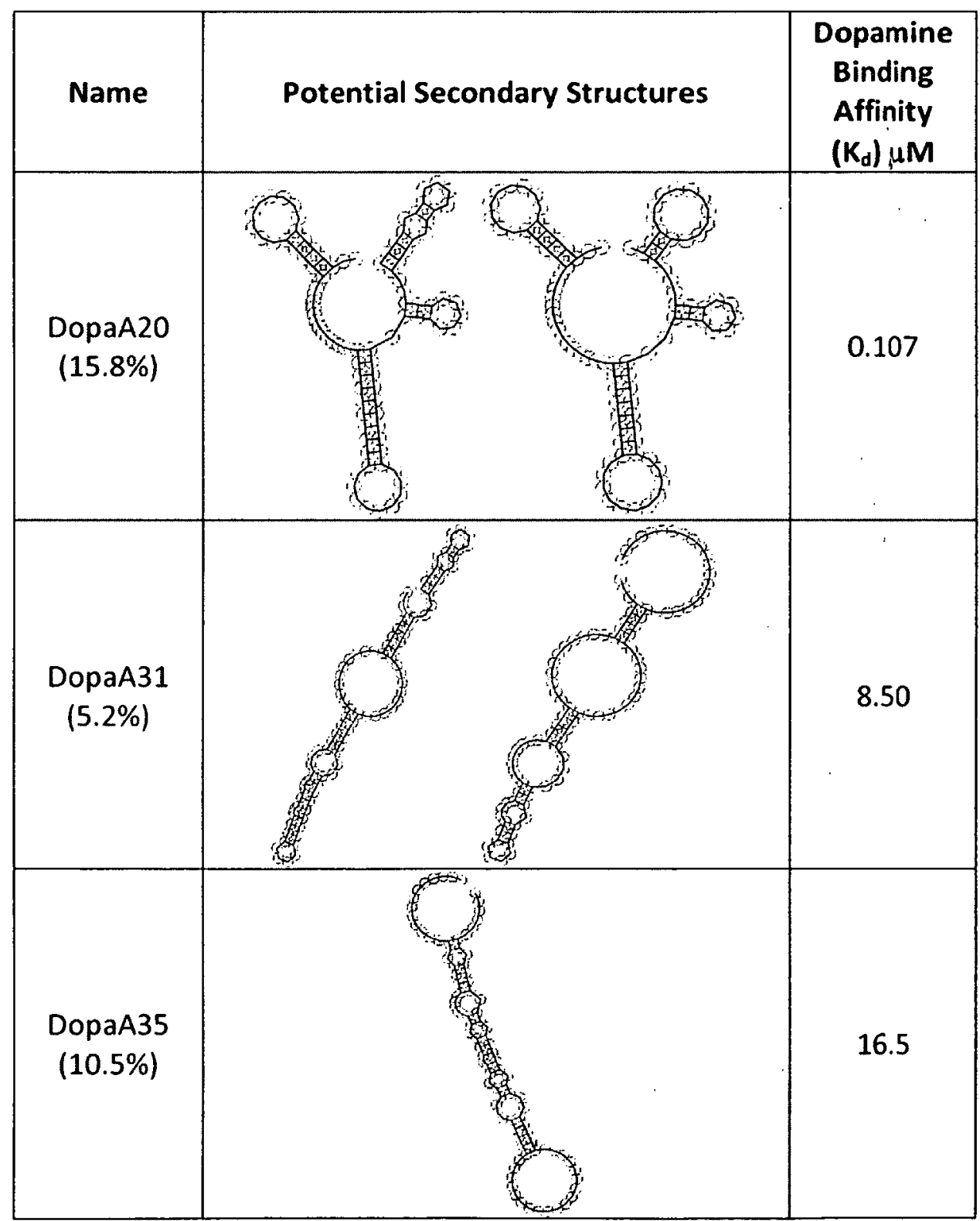




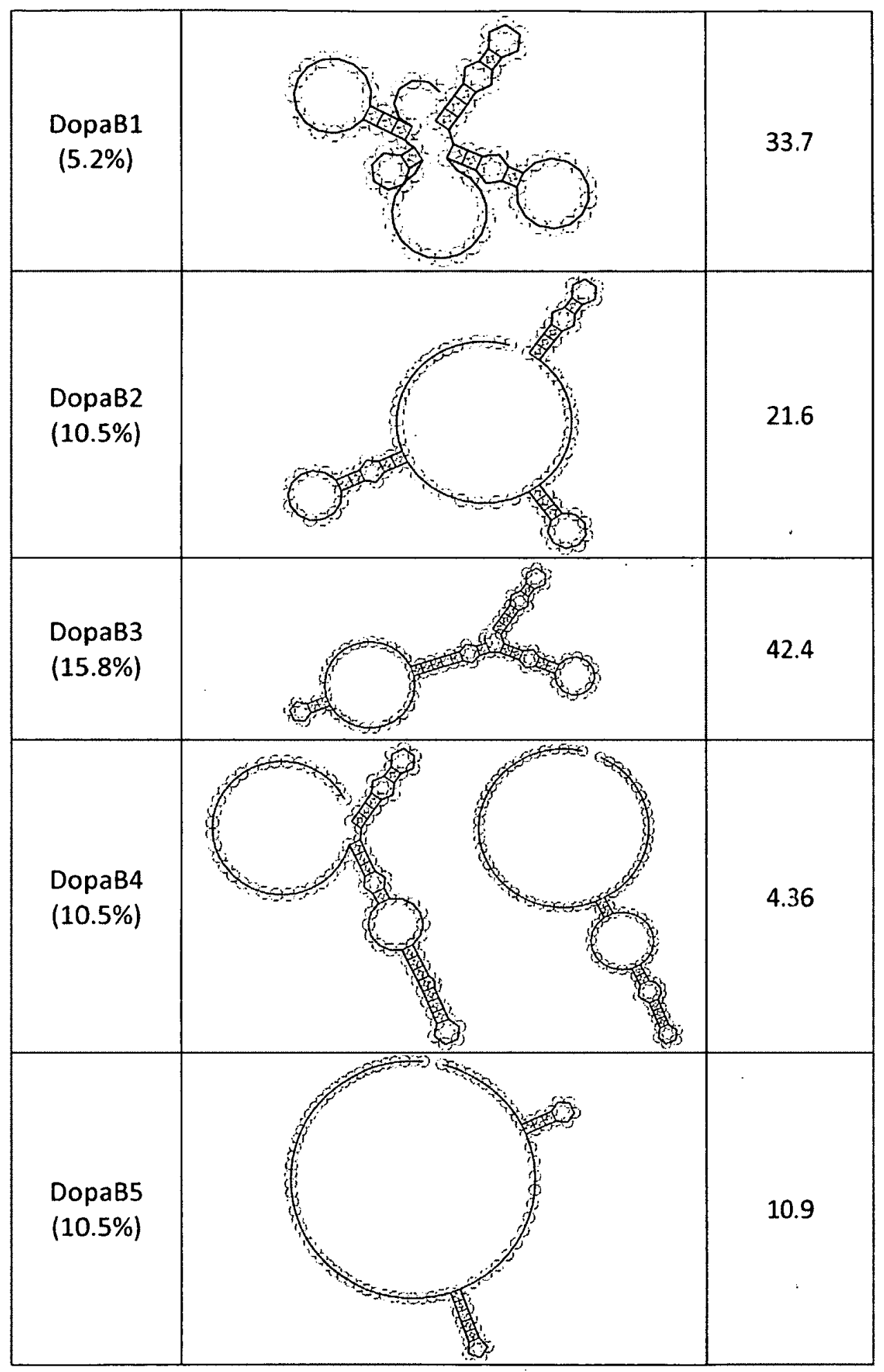




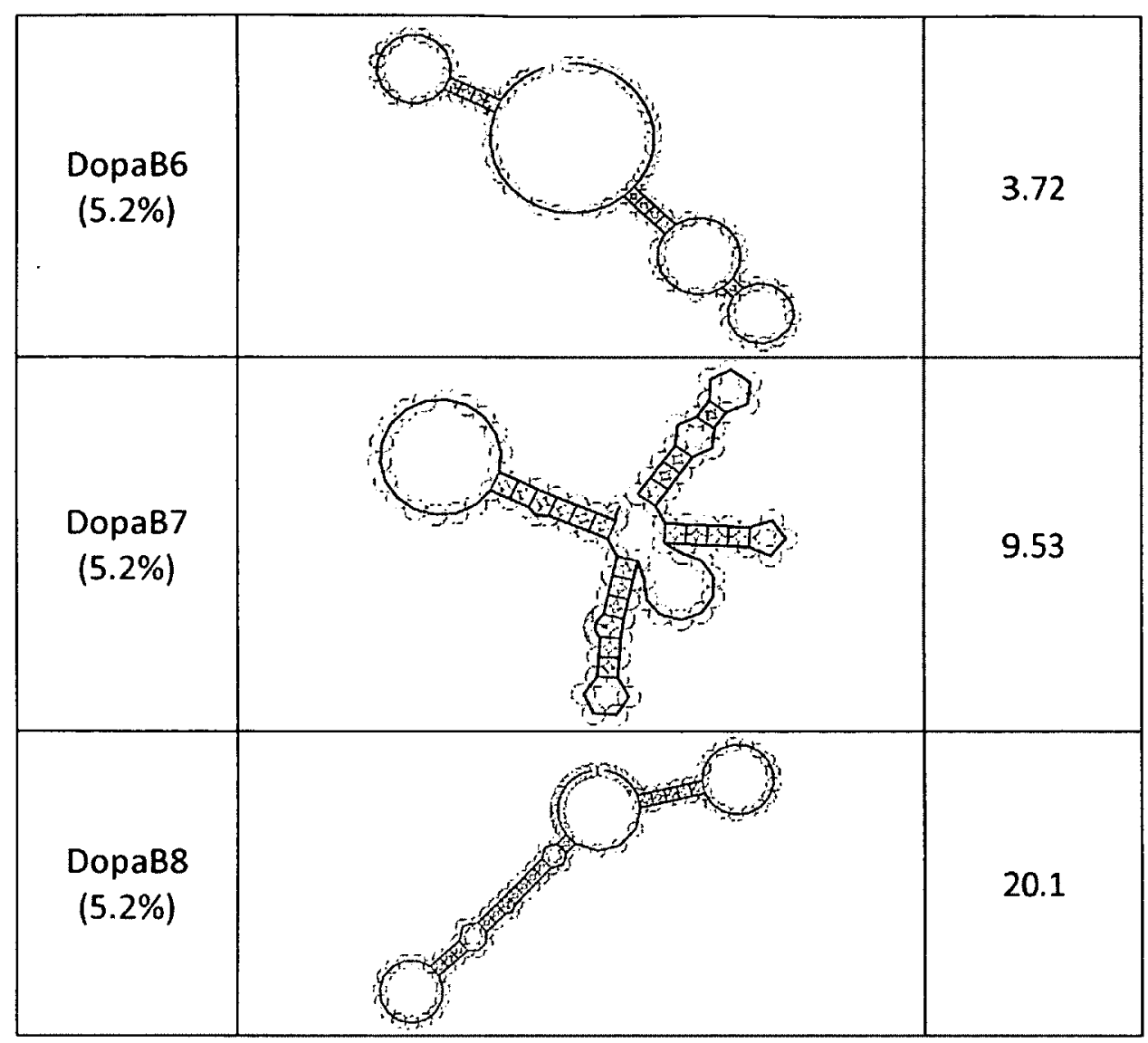




\section{Chapter 5: Systematic Evolution of Ligands by EXponential enrichment (SELEX) for DNA aptamers of microcystin-LR}

This work was done in collaboration with Dr Rocio Aranda-Rodriguez in the Healthy Environments and Consumer Safety Branch of Health Canada. 


\begin{abstract}
Microcystin LR is the most common cyanotoxin produced by fresh water blue-green algae blooms. The hepatotoxic and carcinogenic effects of Microcystin LR make it a molecule of interest for environmental monitoring in water supplies. Current techniques for the detection of this toxin rely on high performance liquid chromatography, liquid chromatography-mass spectrometry and ELISA techniques. Here, we describe the production of DNA aptamers for microcystin LR for the purpose of generating aptamer-based microcystin LR detection kits. Aptamers produced with this selection suggested binding affinities in the nanomolar range and are currently being investigated for their suitability for microcystin LR detection.
\end{abstract}




\section{Introduction}

Microcystins are known hepatotoxins and susnected carcinogens which are produced as secondary metabolites by freshwater cyanobacteria (Campos and Vasconcelos 2010; Pearson et al., 2010). While cyanobacteria, also known as "bluegreen algae", are found worldwide, excessive growth, known as blooms, produced by agricultural pollution has been recognized as a significant health concern for people, livestock and wildlife (Amorim and Vasconcelos, 1999). It has been suggested that bloom size is regulated by microcystins such that they promote colony growth and play a role in the persistence of the blooms (Gan et al., 2012). As such, environmentally relevant concentration during blooms can range from $0.25-10 \mu \mathrm{g} / \mathrm{L}$ (Gan et al., 2012). However, even without bloom formation cyanobacteria have been observed to contaminate drinking water treatment plants, producing concentrations of microcystins in treated water of $2.47 \mu \mathrm{g} / \mathrm{L}$ (Zamyadi et al., 2012).

Over 80 variants of microcystin have been characterized with microcystin LR being the most common isoform (Figure 5.1; Pearson et al., 2010). Microcystin toxicity has been observed to be as low as $50 \mu \mathrm{g} / \mathrm{Kg}$, LD50 for microcystin LR in mouse models (Krishnamurthy et al., 1986) and epidemiological studies have linked microcystins exposure from drinking water to liver and colorectal cancer (Svircev et al., 2009; Zhou and Chen, 2002). Therefore, detection methods have had to be both highly sensitive and specific with the World Health Organization setting provisional values of $1 \mu \mathrm{g} / \mathrm{L}$ microcystin LR as a guideline for safe drinking-water quality. Conventional detection methods rely on high performance liquid chromatography, liquid chromatography-mass 


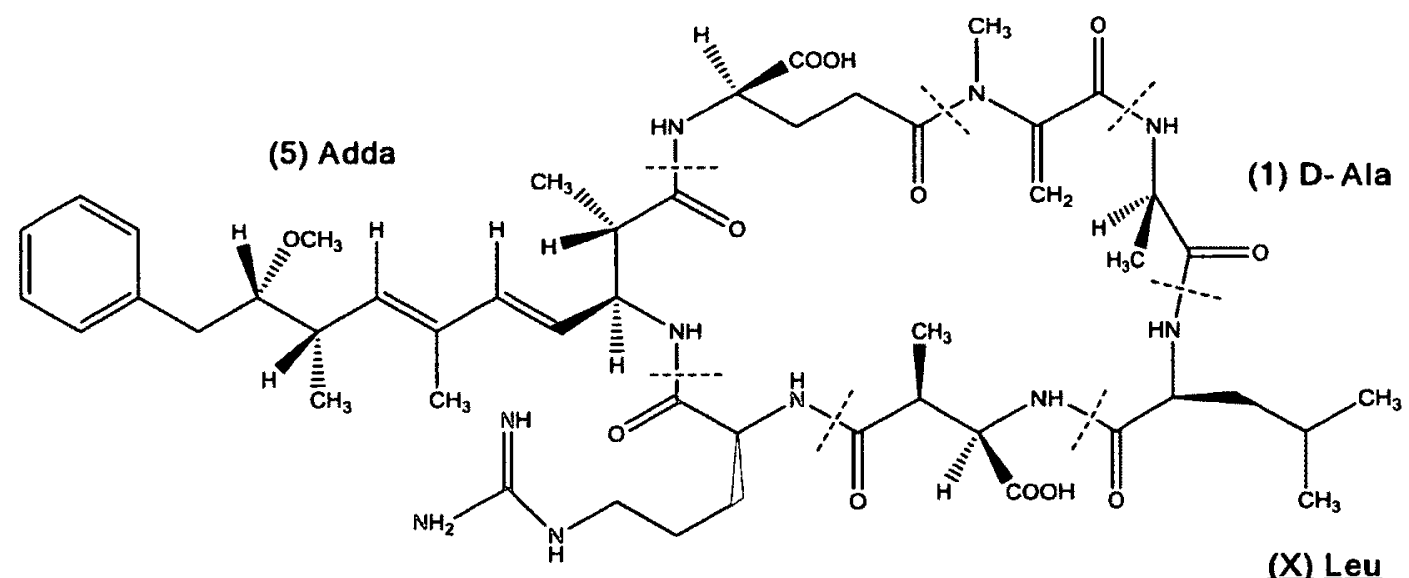

A

(2) Arg

(3) D-MeAsp

(X) Leu

(6) D-Glu

(7) Mdha

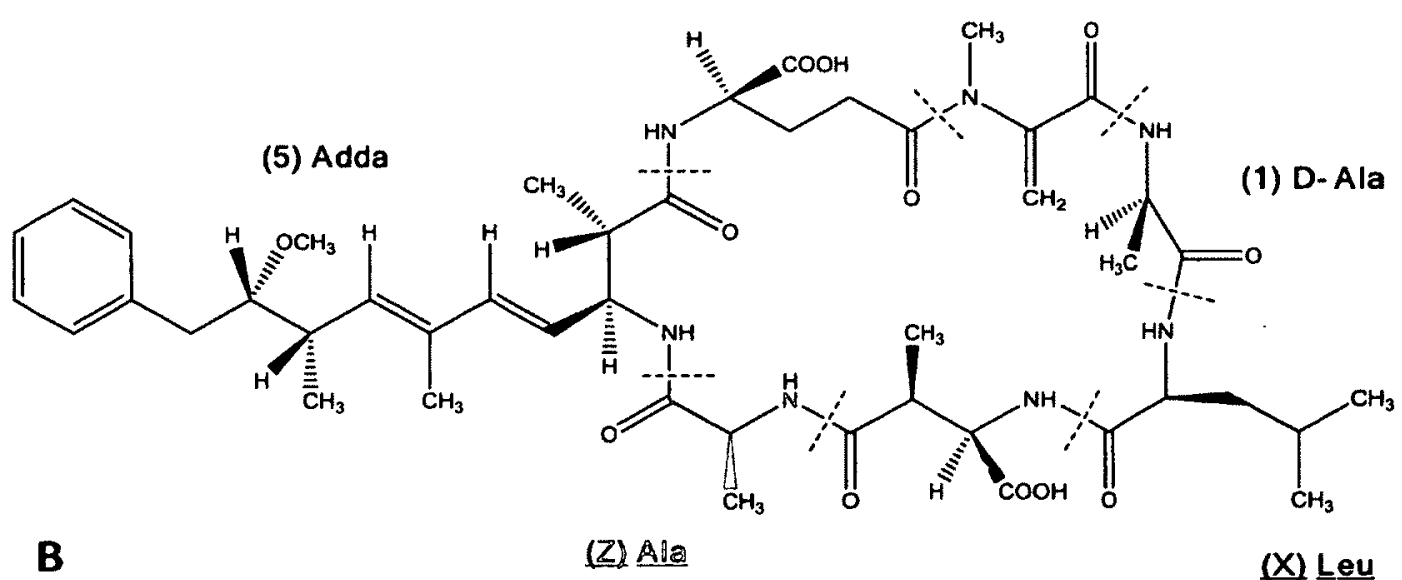

(3) D-MeAsp

Figure 5.1. Microcystins are cyclic heptapeptides which are produced by cyanobacteria. Over 80 variants of these molecules have been reported but are distinguished mainly by their amino acid substituent's in position $X$ and $Z$ of the ring (Campos and Vasconcelos, 2010). Microcystin LR (A) which is the most common form of these microtoxins has an arginine in position $Z$ and a leucine at position $X$. Microcystin LA (B) is distinguishable from microcystin LR by the presence of an alanine in position $Z$. 
spectrometry and enzyme-linked immunosorbent assay (ELISA) techniques (Msagati et al., 2006).

These methods vary in their sensitivity and selectivity with mass spectrometry having the best detection limits $(\sim 0.02 \mu \mathrm{g} / \mathrm{L}$; Msagati et al., 2006) although immunosensors kits, in development, are quickly approaching the same level of sensitivity ( $\sim 0.06 \mu \mathrm{g} / \mathrm{L}$; Herranz et al., 2012). Innovation in portable detection platforms has been spurred by a need for rapid,mobile, cost effective and reliable point of sampling detection systems. For example, Abraxis has recently released a field test strip kit which has been based on competitive antibody binding (Humpage et al., 2012). This kit has a narrow detection range of to $1-10 \mu \mathrm{g} / \mathrm{L}$ and a high degree of cross reactivity to microcystin analogues but allows for fairly reliable real time monitoring of microcystins (Humpage et al., 2012). There has also been some previous work on aptamer based microcystin LR based detection systems. Gu and Famulok (2004) have looked into the production of RNA aptamers for the detection of microcystin LR, reporting binding around $0.5 \mu \mathrm{M}$, but did not publish the sequences for the aptamers. Nakamuraa et al., (2001) examined the production and use of DNA aptamers with surface plasmon resonance for microcystin LR detection. Their work produced a non-selective biosensor, which, based on the detection range $(50 \mu \mathrm{g} / \mathrm{mL}-1 \mathrm{mg} / \mathrm{mL})$, they concluded was not an improvement over current detection methods.

This study looks to improve on the previous aptamer microcystin LR studies both in selectivity and sensitivity with the goal of producing portable, inexpensive and reliable detection systems for this cyanotoxin. 


\section{Materials and methods:}

5-Bromo-4-chloro-3-indoyl- $\beta$-D-galactopyranoside (X-gal), Cyanogen bromide activated Sepharose ${ }^{\circledast} 4$ Fastflow lyophilized powder were purchased from Sigma-Aldrich. The MC-LR Sepharose column was obtained from Abkem Iberia S.L. Microcystin LR and microcystin LA were acquired from Alexis Biochemicals. The ELISA QuantiPlate Kit for Microcystins was acquired from Envirologix. LB broth was purchased from Bioshop. StrataClone ${ }^{\mathrm{TM}}$ PCR Cloning Kit was purchased from Stratagene. Illustra TempliPhi DNA Sequencing Template Amplification Kit was acquired from GE Healthcare.

\section{DNA Sequences}

Primer 1: 5'-AGACCACAACGGTTTCCC-3'

Primer 2: 5'-CAAGGGGTTATGCTA-3'

Primer 1 with Fluorescein tag: 5'-RAGACCACAACGGTTTCCC-3'

Primer 2 with Poly $A_{20}$ tail and ethyleneglycol spacer:

5'-AAAAAAAAAAAAAAAAAAAAXCAAGGGGTTATGCTA-3'

DNA $P$ ool sequence $N_{50}$ random sequencs: 
$5^{\prime}-$

AGACCACAACGGTTTCCCNNNNNNNNNNNNNNNNNNNNNNNNNNNNNNNNNNNNNNNN

NNNNNNNNNNTAGCATAACCCCTTG-3'

For the above sequences $\mathbf{R}$ represents the Fluorescein label 6-Fluorescein Phosphoramidite, $\mathrm{X}$ represents Spacer Phosphoramidite 18 , the hexaethyleneglycol spacer which prevents TAQ polymerase read through, and $\mathbf{N}$ represents an indeterminate random base in the sequence which could be adenine, thymine, cytosine or guanine.

\section{DNA based selection for microcystin LR aptamers}

The initial pool (approximately $0.3 \mathrm{nmols}$ ) was suspended in $300 \mu \mathrm{L}$ of selection buffer, $50 \mathrm{mM}$ Tris $\mathrm{HCl}(\mathrm{pH} 7.5), 150 \mathrm{mM} \mathrm{NaCl}$ and $2 \mathrm{mM} \mathrm{MgCl}_{2}$. This was heated for 5 minutes at $100^{\circ} \mathrm{C}$ and then cooled to room temperature. A counter selection column and the microcystin functionalized selection columns were prepared by weighing approximately $50 \mathrm{mg}$ of the control Sepharose ${ }^{\circledR} 4$ Fastflow lyophilized powder and approximately $15 \mathrm{mg}$ of the MC-LR Sepharose column into separate micro-spin filter tubes $(0.45 \mu \mathrm{m}$ nylon filters). The columns were washed with $500 \mu \mathrm{L}$ of selection buffer which was removed by centrifugation at $1000 \mathrm{~g}$ for 1 minute. Washing was repeated a further 8 times to ensure the columns were pre-equilibrated for the selection process. The pool was added to the Sepharose column and allowed to shake for 20 minutes before being centrifuged at $1000 \mathrm{~g}$ for a minute. The collected pool was then added to 
the MC-LR Sepharose column and allowed to shake for 20 minutes before being centrifuged at $1000 \mathrm{~g}$ for a minute. The MC-LR Sepharose column was then subjected to two rounds of washing with $500 \mu \mathrm{L}$ of selection buffer which was removed by centrifugation at $1000 \mathrm{~g}$ for 1 minute. DNA sequences still bound to the column were eluted with the addition of $500 \mu \mathrm{L}$ of hot $\mathrm{ddH}_{2} \mathrm{O}\left(\sim 95^{\circ} \mathrm{C}\right)$ which was mixed and centrifuged immediately at $1000 \mathrm{~g}$ for a minute. This elution was repeated and a few crystals of Tris were added to the samples which were then allowed to dry on the Savant DNA120 SpeedVac Concentrator overnight. Samples were then amplified with PCR and the new pool was isolated using gel electrophoresis (see sections below). A portion of the new pool was frozen at $-80^{\circ} \mathrm{C}$ and the rest was prepared for the second round of selection.

The second round of selection followed the same procedures employed during the first round with the exceptions that the counter selection colum was omitted and DNA sequences which bound to the MC-LR Sepharose column were eluted using 4 washes of hot $3.5 \mathrm{M}$ Urea $\left(500 \mu \mathrm{L}\right.$ each, $\left.\sim 90^{\circ} \mathrm{C}\right)$. The eluted samples were initially concentrated then desalted with dd $\mathrm{H}_{2} \mathrm{O}$ three times using 3,000 NMWL Millipore Microcon centrifugal filter devices $12000 \mathrm{~g}$ for 30 minutes, before being dried down for PCR amplification and isolation.

Round three of SELEX reintroduced a $100 \mathrm{mg}$ Sepharose counter selection column. Elution of bound DNA sequences from the MC-LR Sepharose column was achieved using $4500 \mu \mathrm{L}$ additions of $3.5 \mathrm{M}$ Urea in $10 \mathrm{mM}$ Tris buffer, $\mathrm{pH} 7.4$, heated to $\sim 90^{\circ} \mathrm{C}$. The 
samples were then dried and redissolved in a minimal amount of $\mathrm{dd}_{2} \mathrm{O}$ for ethanol precipitation. To $150 \mu \mathrm{L}$ of the DNA sample $75 \mu \mathrm{L}$ of $3 \mathrm{M} \mathrm{NaCl}$ was added, followed by 1 $\mathrm{mL}$ of anhydrous ethanol. The mixture was incubated at $-80^{\circ} \mathrm{C}$ for 20 minutes before centrifugation $10000 \mathrm{~g}$ for 20 minutes. The supernatant was removed the samples dried in the speedvac and samples were then redissolved in $\mathrm{ddH}_{2} \mathrm{O}$ for desalting using 3,000 NMWL Millipore Microcon centrifugal filter devices $12000 \mathrm{~g}$ for 30 minutes, before PCR amplification and isolation.

Round four of SELEX did not introduce any changes to the procedure used in the third round except that the Sepharose counter selection column was increased to 150 mg.

Round five of SELEX introduced a fractionation of the eluted DNA binding to the MC-LR Sepharose column by initially eluting with two addition of $500 \mu \mathrm{L}$ of hot dd $\mathrm{H}_{2} \mathrm{O}$ $\left(\sim 95^{\circ} \mathrm{C}\right)$ followed by $2500 \mu \mathrm{L}$ additions of $3.5 \mathrm{M}$ Urea in $10 \mathrm{mM}$ Tris buffer, $\mathrm{pH} 7.4$, heated to $\sim 90^{\circ} \mathrm{C}$.

Round six of SELEX introduced an additional counter selection technique as the pool was mixed with a homolog of microcystin LR, microcystin LA at a ratio of 100:1 with the pool before it was passed through the counter selection column and the MC-LR Sepharose column. Elution was carried out with the $3.5 \mathrm{M}$ Urea in $10 \mathrm{mM}$ Tris buffer, $\mathrm{pH} 7.4$, heated to $\sim 90^{\circ} \mathrm{C}$ only. Samples from this selection were amplified using PCR and unmodified primers into double stranded DNA for cloning and sequencing (see below). 
PCR

Samples to be amplified were suspended in reaction buffer consisting of $100 \mathrm{mM}$ Tris $\mathrm{HCl} \mathrm{pH} \mathrm{9,50} \mathrm{mM} \mathrm{KCl,} 1 \%$ Triton X-100, $2 \mathrm{mM} \mathrm{MgCl}, 0.5 \mathrm{mM}$ dNTPs, $1 \mu \mathrm{M}$ of each primer and $5 \mathrm{U}$ of Taq DNA polymerase in a final volume $100 \mu \mathrm{L}$. Samples were amplified using an Eppendorf Mastercycler EP Gradient, Thermal Cycler programmed to initially denature samples at $95^{\circ} \mathrm{C}$ for 2 minutes followed by 30 rounds of amplification. Amplification cycles consisted of primer annealing at $57^{\circ} \mathrm{C}$ for 1 minute, followed by 1 minute of template amplification $72^{\circ} \mathrm{C}$ and concluding with sample denaturation for 30 seconds at $95^{\circ} \mathrm{C}$ before initiation of the following amplification cycle. The amplification process concluded with an extended 5 minute $72^{\circ} \mathrm{C}$ template amplification phase before samples were cooled to $4^{\circ} \mathrm{C}$ before processing. Amplified samples were dried down using a Savant DNA120 SpeedVac Concentrator, Thermo Electron Corporation, in preparation for electrophoretic isolation.

\section{Polyacrylamide Gel Electrophoresis}

Samples were isolated with $12 \%$ acrylamide gels cast using $18 \times 16 \mathrm{~cm}$ gel plates. Twelve percent acrylamide gels were cast by combining $31.5 \mathrm{~g}$ of urea, $23.5 \mathrm{mLs}$ of 5.3 $\mathrm{M}$ acrylamide and $130 \mathrm{mM} \mathrm{N}, \mathrm{N}^{\prime}$-methylenebisacrylamide, $15 \mathrm{mLs}$ of $0.44 \mathrm{M}$ Boric acid, $0.44 \mathrm{M}$ Tris, and $10 \mathrm{mM}$ EDTA with $14 \mathrm{mLs} \mathrm{dH}_{2} \mathrm{O}$. The mixture was heated to $37^{\circ} \mathrm{C}$ filtered and allowed to cool to room temperature. This was followed by the addition of $0.45 \mathrm{mLs}$ of $10 \%$ ammonium persulfate followed by $35 \mu \mathrm{L} \quad \mathrm{N}, N^{\prime}, N^{\prime}, N^{\prime}-$ 
tetramethylethylenediamine. The gels were poured and allowed to polymerize for 20 minutes. After polymerization, the gels were set up in a Hoefer SE 600 series vertical slab gel electrophoresis unit and the buffer reservoirs were filled with $88 \mathrm{mM}$ Boric acid, $88 \mathrm{mM}$ Tris, and $2 \mathrm{mM}$ EDTA. Gels were pre-equilibrated typically using a constant current of 35 milliamps for 20 mins using a FisherBiotech Power Supply FB1000. DNA samples were dissolved in minimal amounts of $\mathrm{dH}_{2} \mathrm{O}$ and mixed with an equal amount of formamide before being heated at $55^{\circ} \mathrm{C}$ for 5 minutes. This was followed by loading onto the gel for separation. The gels were subjected typically to a constant current of 35 milliamps for 2 hours.

Upon completion of the electrophoretic run, the gels were imaged using an Alpha Innotech imager. Bands of interest were cut out and the gel crushed and suspended in 5 times the volume of $10 \mathrm{mM}$ Tris $\mathrm{pH}$ 7.4. Samples were frozen using liquid nitrogen followed by heating to $90^{\circ} \mathrm{C}$ for 10 minutes. The samples were then allowed to mix and incubated overnight at $37^{\circ} \mathrm{C}$. Following incubation, the samples were filtered $(0.45 \mu \mathrm{m})$ to remove the remaining gel fragments. Samples were then lyophilized to reduce the sample volume followed by resuspension in a minimal volume of deionized water for desalting and concentration. Samples were concentrated initially followed by 4 rounds of deionization with deionized water using 3,000 NMWL Millipore Microcon centrifugal filter devices $12000 \mathrm{~g}$ for 30 minutes. Samples were then quantified by assessing their absorbance at $260 \mathrm{~nm}$ and lyophilized. 


\section{Cloning and Sequencing}

Sequences of interest were cloned using the StrataClone PCR cloning kit. Double stranded DNA samples isolated from the gel were desalted, dried and resuspended in 50 $\mu \mathrm{L}$ and the concentration checked to assure it fell within the range of $5-50 \mathrm{ng} / \mu \mathrm{L}$. The sample and reaction components were kept on ice prior to the combination of $2 \mu \mathrm{L}$ of the sample and $3 \mu \mathrm{L}$ of the provided StrataClone Cloning Buffer. This was followed by the addition of $1 \mu \mathrm{L}$ of the StrataClone Vector Mix amp. The sample was then mixed with the pipette and allowed to react for 5 minutes at room temperature. The reaction mixture containing the recombinate plasmids was then placed on ice $\left(4^{\circ} \mathrm{C}\right)$ again prior to cell transformation. One tube of StrataClone Solopack competent cells was thawed at $4^{\circ} \mathrm{C}$ prior to the addition of $1 \mu \mathrm{L}$ of the reaction mixture. The sample was gently mixed through inversion before incubation on ice for 20 minutes. The sample was then subjected to heat shock by incubating it at $42^{\circ} \mathrm{C}$ for 5 minutes followed by a further 2 minutes on ice. This was followed by the addition of $250 \mu \mathrm{L}$ of LB medium at $42^{\circ} \mathrm{C}$ and incubation of the mixture at $37^{\circ} \mathrm{C}$ with continual mixing for an hour. A LB-ampicillin plate ( $1 \% \mathrm{NaCl}, 1 \%$ tryptone, $0.5 \%$ yeast extract, $2 \%$ agar and $100 \mu \mathrm{g} / \mathrm{mL}$ ampicillin) was prepared for cell culturing with the addition of $40 \mu \mathrm{L}$ of $2 \% \mathrm{X}$-gal in dimethylformamid. Once the plates were prepared, $50 \mu \mathrm{L}$ of the transformed cell suspension was plated onto the plates and the plates were incubated overnight at $37^{\circ} \mathrm{C}$.

The following day white colonies were selected for insert sequencing. Plasmids were amplified using the TempliPhi DNA amplification kit. Colonies were gently touched with a pipette tip to transfer a few of the cells to $5 \mu \mathrm{L}$ reaction buffer. Samples were 
then heat shocked at $95^{\circ} \mathrm{C}$ for 3 minutes to release the desired plasmid followed by cooling to $4^{\circ} \mathrm{C}$. In a separate tube, at a mix ratio of $5 \mu$ l of reaction buffer to $0.2 \mu$ l of the Phi polymerase enzyme mix, the enzyme was prepared for addition to the samples which were going to be amplified. Once the shocked cell samples had cooled $5 \mu \mathrm{L}$ of the Templiphi enzyme mix was added and incubated at $30^{\circ} \mathrm{C}$ for 18 hours. After the 18 hour incubation samples were heated to $65^{\circ} \mathrm{C}$ for 10 minutes to inactivate the polymerase before they were stored at $4^{\circ} \mathrm{C}$.

Samples were sent to the University Core DNA Services at the University of Calgary for sequencing.

\section{Anisotropy studies with fluorescein labelled aptamer}

Anisotropy studies of fluorescein labelled potential aptamer sequences were performed by incubating the sequences at constant concentration in increasing concentrations of microcystin LR. All samples were prepared in $50 \mathrm{mM}$ Tris $\mathrm{HCl}(\mathrm{pH} 7.5)$, $150 \mathrm{mM} \mathrm{NaCl}, 2 \mathrm{mM} \mathrm{MgCl} 2$. Fluorescent data was collected with a $40 \mu \mathrm{L}$ fluorescent cell with a FluoroLog-3 Spectrofluorometer from HORIBA Jobin Yvon (Ex/Em = 498/519 nm).

\section{ELISA competitive binding experiments}

Analysis of the binding affinity between the aptamer sequences and microcystin LR was assessed using the Envirologix, QuantiPlate Kit for Microcystins-EP-022, an Enzyme- 
Linked Immuno Sorbent Assay (ELISA). Assays were performed using a modification of the provided protocol in which $50 \mu \mathrm{L}$ of the microcystin LR aptamer selection buffer, 50 $\mathrm{mM}$ Tris- $\mathrm{HCl} \mathrm{pH} \mathrm{7.4,} 140 \mathrm{mM} \mathrm{NaCl}, 2 \mathrm{mM} \mathrm{MgCl}_{2}$, was added to each assay well followed by the addition of $50 \mu \mathrm{L}$ of test samples or controls solutions to individual wells. The assay plates were incubated for 30 minutes on a shaker at room temperature followed by washing the wells 5 times with the provided washing solution. After removal of the wash solution, $50 \mu \mathrm{L}$ assay diluent, $50 \mu \mathrm{L}$ distilled water and $100 \mu \mathrm{L}$ microcystin $\mathrm{LR}$ horseradish peroxidase (MC-LR-HRP) conjugate were added and incubated while shaking at room temperature for 30 minutes. This was followed by another round of

washing, 5 times with the provided wash solution. Horseradish peroxidase activity was analysed with the addition of $100 \mu \mathrm{L}$ of provided 3, 3', 5, 5'-tetramethylbenzidine (TMB) substrate solution followed by incubation while shaking at room temperature for 30 minutes. Reactions were stopped with the addition of $100 \mu \mathrm{L}$ of $1 \mathrm{M} \mathrm{HCL}$ and then analysed on a Thermo Multiskan Spectrum plate reader at $450 \mathrm{~nm}$.

\section{Circular Dichroism}

Circular dichroism measurements were performed by initially heating the aptamer sample to $90^{\circ} \mathrm{C}$ then cooling of the potential aptamers stock solution followed by mixing $1 \mathrm{~mL}$ of the stock with $1 \mathrm{~mL}$ of microcystin-LR to produce a final concentration of $5 \times 10^{-7}$ $M$ for the DNA and $1.7 \times 10^{-5} \mathrm{M}$ microcystin LR in a $1 \mathrm{~cm}$ quartz cuvette. This was accompanied by the preparation of a $5 \times 10^{-7} \mathrm{M}$ DNA working mixture by diluting the 
stock solution by half. All solutions were prepared in the selection buffer $50 \mathrm{mM}$ Tris$\mathrm{HCl} \mathrm{pH} 7.4,140 \mathrm{mM} \mathrm{NaCl}, 2 \mathrm{mM} \mathrm{MgCl}$. Serial dilutions of the microcystin LR concentration were performed by removing $1 \mathrm{~mL}$ of the DNA microcystin LR mixture and replacing it with $1 \mathrm{~mL}$ of the $5 \times 10^{-7} \mathrm{M}$ DNA sample between readings. Samples were scanned 210 to $320 \mathrm{~nm}$ on a Jasco J-810 spectrometer.

\section{Data fitting for dissociation constant determination}

Dissociation constants and binding curve fits were determined by fitting the observed data to binding equations using the solver feature of Microsoft Excel (Fylstra et al., 1998; Nenov and Fylstra 2003; Kemmer and Keller 2010).

\section{Results and discussion}

The selection process for the microcystin LR aptamer used the same selection buffer as that described by Gu and Famulok (2004) who selected for an RNA microcystin LR aptamer. Although from their selection process they reported a $500 \mathrm{nM}$ binding affinity for a cloned sequence they designated MC25, none of the sequences they discovered were published. Similar to the dopamine selection in the previous chapter, the primers were modified according to the Flumag protocol described by Stoltenburg et al., (2005). The selection rounds generally followed the same procedure although several small changes were implemented to improve the affinity of the pool for microcystin LR. For example, taking into account that hot water might not be sufficient 
to elute the highest affinity sequences from the selection column $3.5 \mathrm{M}$ urea was introduced to the elution solution during the second round of selection. The differential elution profile produced by the hot water and urea solutions was additionally exploited in round 5 of the selection where an initial elution with hot water was used to remove less stable binding sequences from the column before urea was used to isolate stronger binding sequences (figure 5.2). The sixth and final round of SELEX was also changed to ensure a high level of specificity for microcystin LR, by preincubating the DNA pool with 100 times its concentration of microcystin LA before it was exposed to the microcystin LR column (Figure 5.3). The use of the high concentration of microcystin LA was used to separate the pool into sequences which bound specifically to the arginine portion of the microcystin LR rather than the portions of the molecule comnon to all the forms of microcystin.

Sequencing of the pool produced a variety of sequences with very little duplication, except for sequence $\mathrm{H} 18$ which appeared twice and $\mathrm{H} 12$ which appeared three times (Figure 5.4). Sequencing of the pool also produced another surprise as the initial pool used in the selection contained a random region of 50 nucleotides in length but the sequenced pool was found to contain deletions of the random region up to 18 nucleotides. Very few of the sequences were observed to retain the initial 82 base length of the original pool and one of the sequences was even observed to contain a 


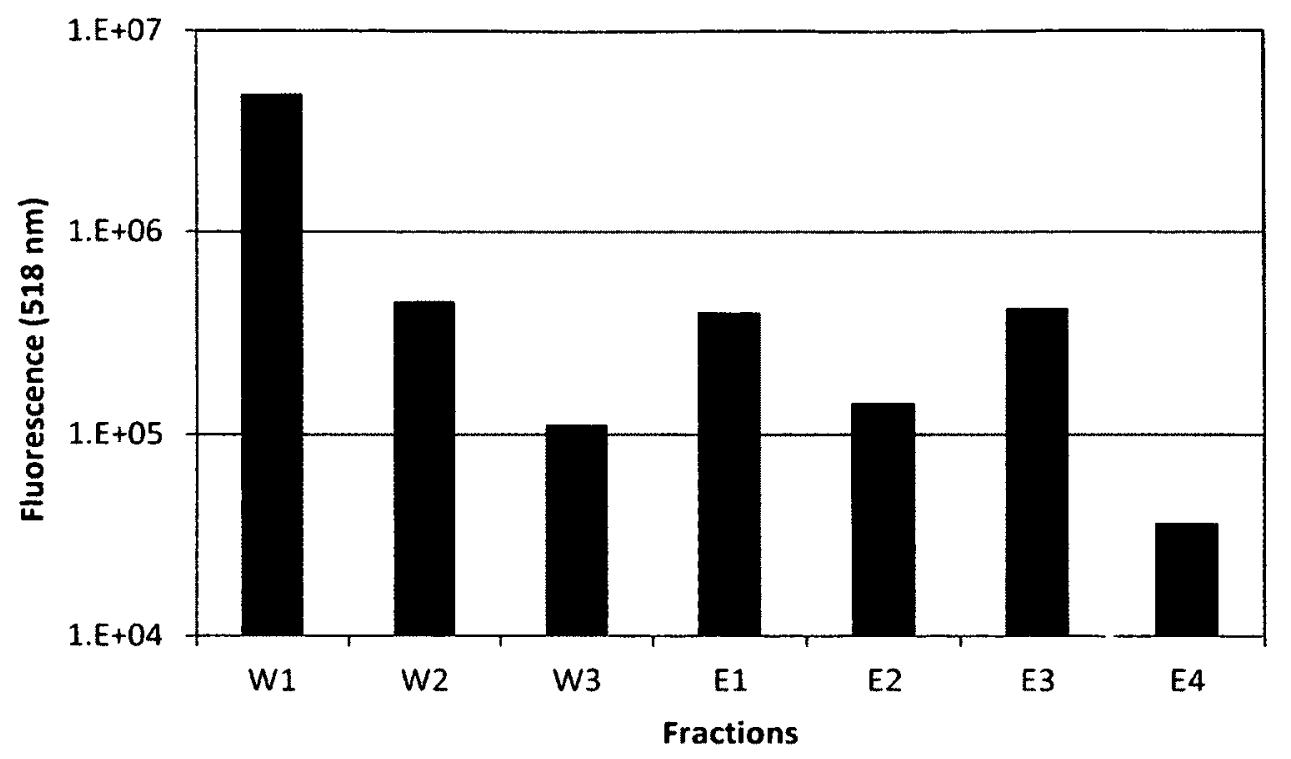

Figure 5.2. Elution profile from the $5^{\text {th }}$ selection round where the pool was initially washed from the microcystin LR column using selection buffer (W1-W3), followed by elution with hot water $\sim 90^{\circ} \mathrm{C}(E 1, E 2)$ then elution with hot $3.5 \mathrm{M}$ Urea $\sim 90^{\circ} \mathrm{C}(\mathrm{E3}, \mathrm{E} 4)$. Bars represent the fluorescence of the collected fractions (excitation 490 emission $518 \mathrm{~nm})$. 


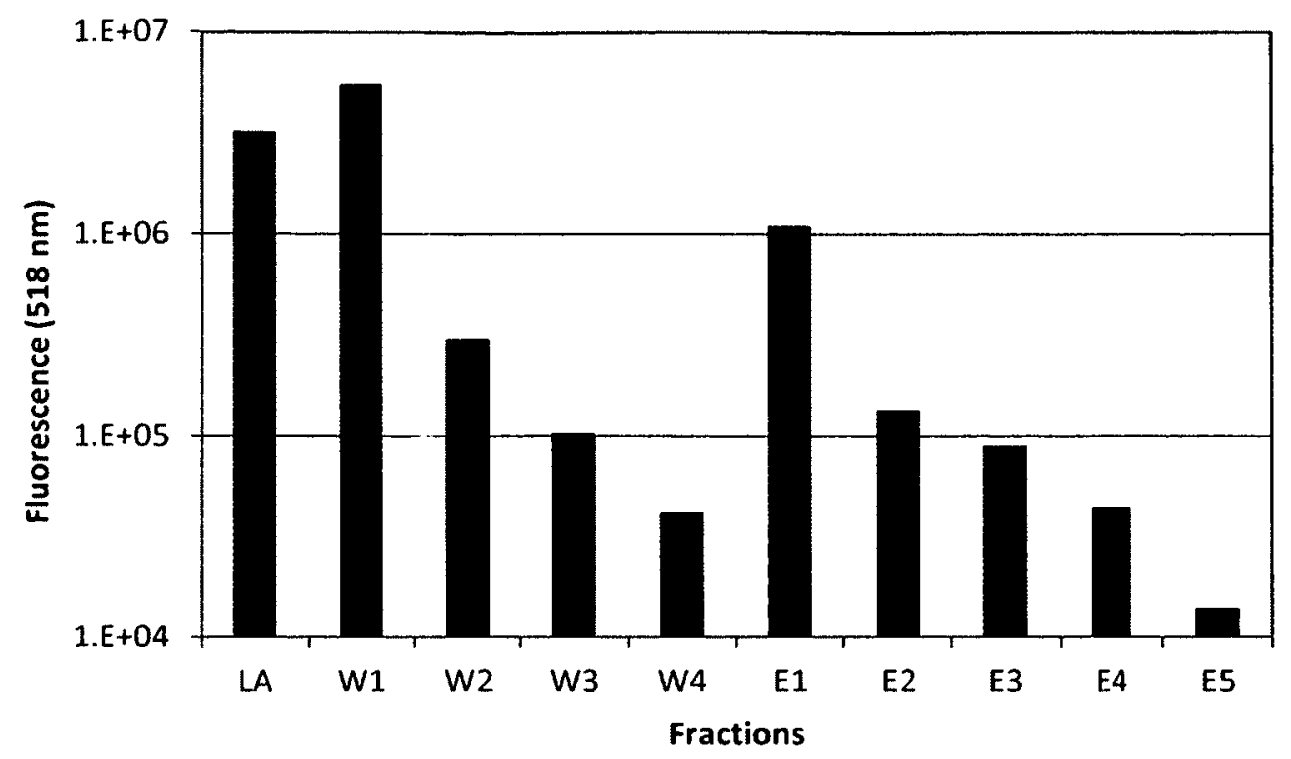

Figure 5.3. Elution profile from the $6^{\text {th }}$ selection round where the pool was initially incubated with 100 fold excess microcystin LA before being exposed to the microcystin LR column. Fluoresces associated with the wash through (LA) followed by washes with the selection buffer (W1-W4) and then elution with hot $3.5 \mathrm{M}$ Urea $\sim 90^{\circ} \mathrm{C}$ (E1-E5). Bars represent the fluorescence of the collected fractions (excitation 490 emission $518 \mathrm{~nm}$ ). 

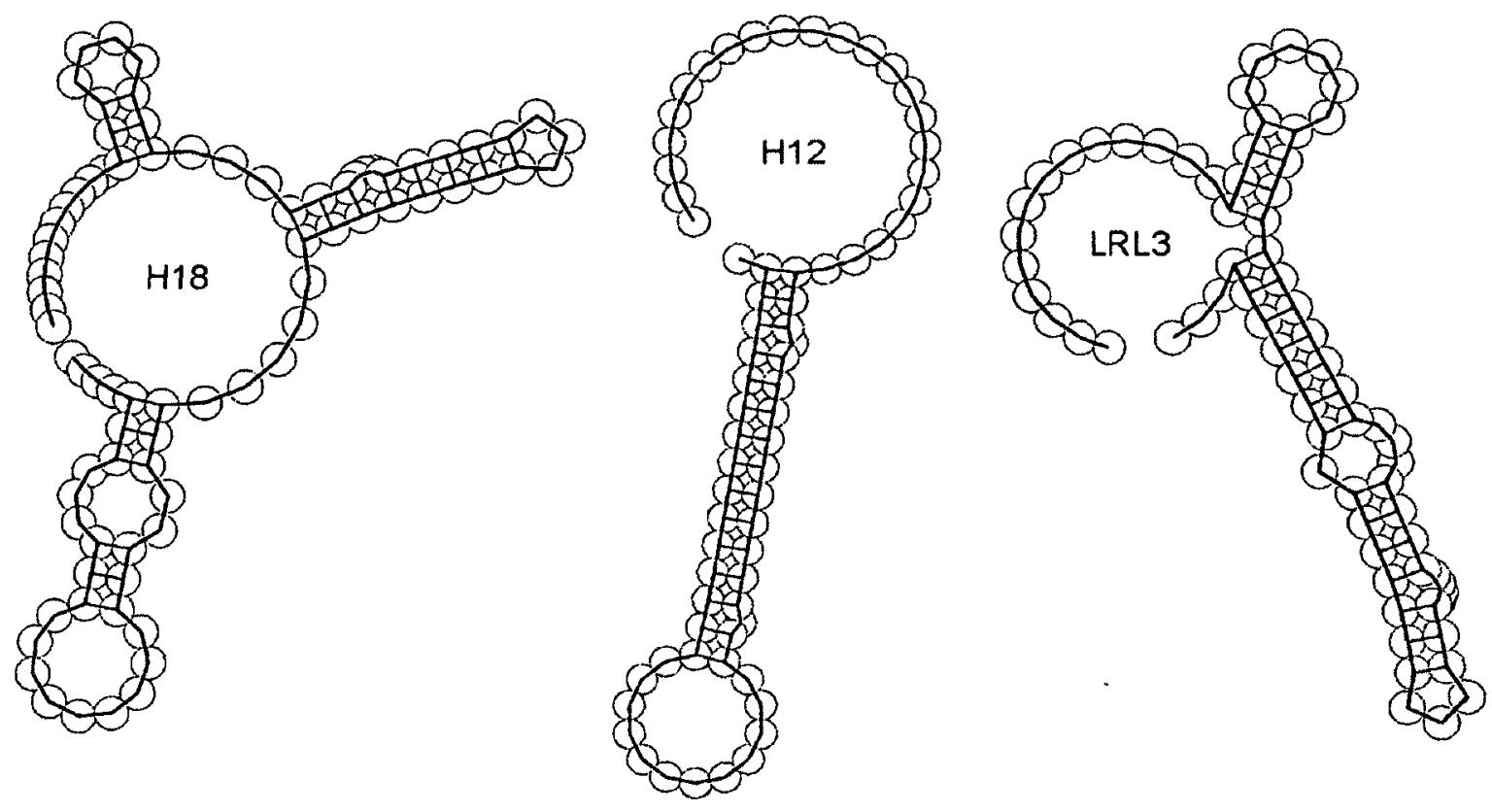

Figure 5.4. Potential secondary structures, primary sequence not shown, for the three SELEX generated DNA sequences used in assessing the pools binding specificity towards microcystin LR. Structures were generated using the DNA settings of the RNAfold suite of the Vienna RNA Websuite (Gruber et al., 2008). 
base insertion producing an 83 base sequence. Indeed, even $\mathrm{H} 18$ at 80 bases and $\mathrm{H} 12$ at 68 bases were reduced in length when compared to the starting pool.

In addition to $\mathrm{H} 18$ and $\mathrm{H} 12$, an additional sequence LRL3 was selected for further microcystin LR binding affinity characterization (Figure 5.4). Initially, based on the success of anisotropy with the dopamine study, fluorescein labeled H12 was examined for its affinity towards microcystin LR (Figure 5.5). Unfortunately, similarly to the dopamine anisotropy study where the aptamer was labeled with fluorescein, a high level of noise due to the small signal change resulting from the relatively low change in rotational momentum lead to concern on the validity of the binding constant (Table 5.1).

The difficulty associated with determining the binding affinity between the DNA sequences and microcystin LR lead to further investigation with circular dichroism. These experiments involved a full spectral scan of the DNA as the concentration of microcystin LR was varied. This allowed for assessment of regions of the spectra which may be susceptible to fluctuation attributable to the concentration of microcystin LR. Fluctuation around $220 \mathrm{~nm}$ seemed to be constant among the sequences tested (Figure 5.6), therefore peak smoothing for binding curve analysis was attempted in this region. Unfortunately, a high degree of noise also reduced confidence in the ability of this technique to accurately determine the binding affinity between the DNA sequences and microcystin LR (Table 5.1). While circular dichroism did suggest that $\mathrm{H} 12$ did have a binding affinity in the nanomolar range as observed with the anisotropy study the 


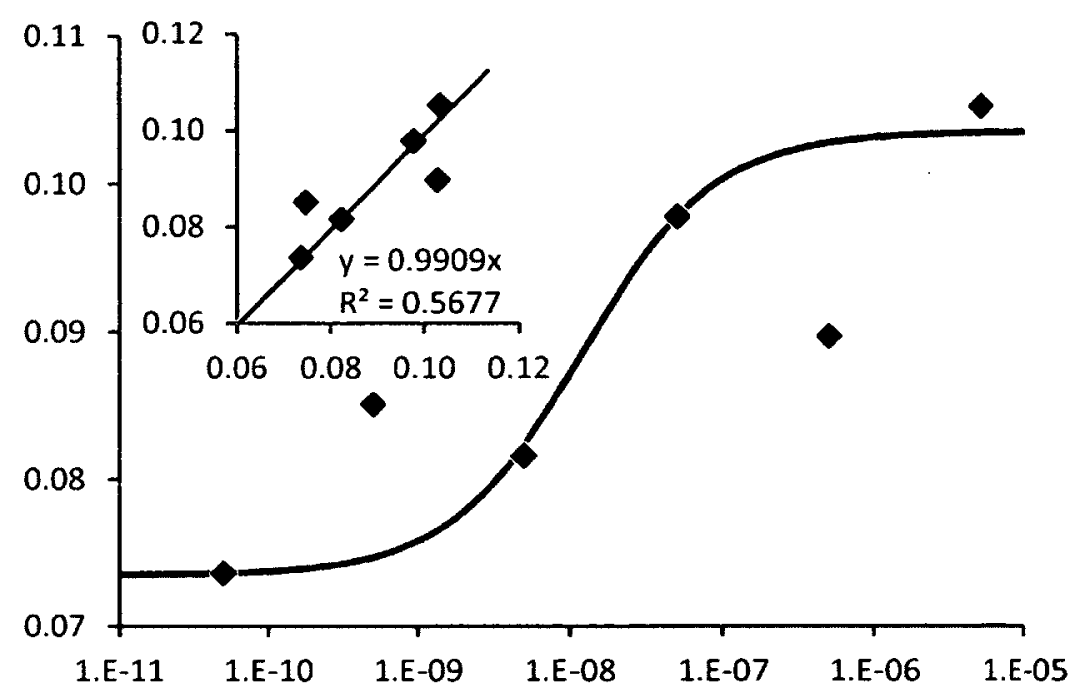

Figure 5.5. Fluorescent anisotropy binding curve for the fluorescently tagged $\mathrm{H} 12$ sequence and microcystin LR. Anisotropy values were collected using $0.57 \mathrm{nM} \mathrm{H} 12$ which was exposed to increasing concentrations of microcystin $\operatorname{LR}(0.05,0.5,5,50,500$, $5000 \mathrm{nM})$. The inset shows the agreement between the observed and the calculated anisotropy values. 

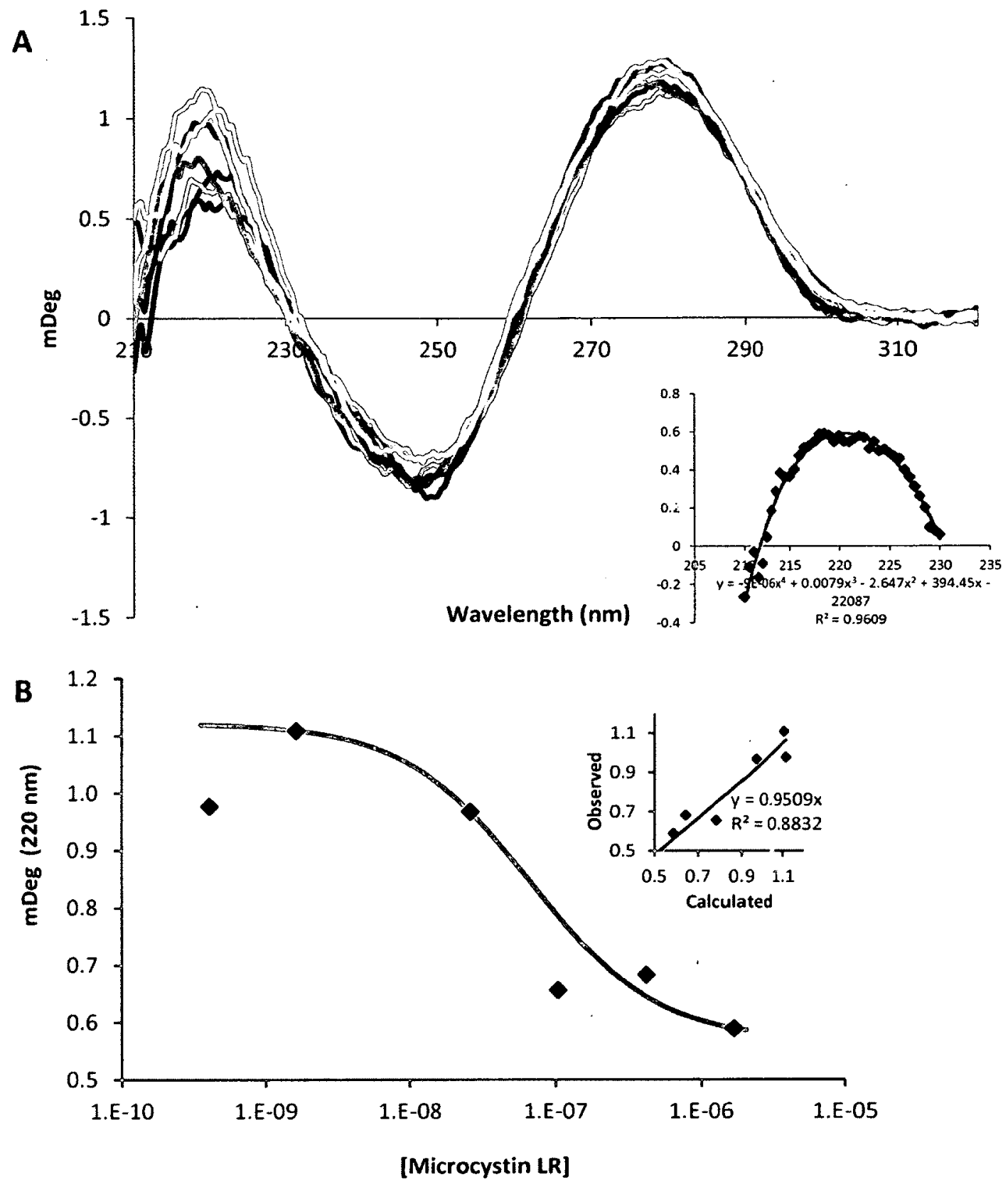

Figure 5.6. CD study of the affinity of $\mathrm{H} 12$ for microcystin $L R(A)$ repetitive scans of $H 12$ with the concentrations of microcystin LR varying $0.4 \mathrm{nM}$ to $1.6 \mu \mathrm{M}$. The insert represents the polynomial smoothing used to analyzing the peak around $220 \mathrm{~nm}$. Binding isotherm fit to the observed data with inset showing the agreement between the observed and the calculated experimental data. 
binding affinities differed by a factor of $6 x$ and the standard deviation of each value was exceedingly high. Additionally, circular dichroism was unable to provide useful information relating to the affinity of LRL3 for microcystin LR (Table 5.1).

To further examine the binding of the DNA sequences, competitive ELISA assays were undertaken. An antibody-based microcystin LR ELISA kit was acquired from Envirologix and used to characterize the binding affinity between the DNA sequences and microcystin LR. In this competitive assay, known concentrations of microcystin LR was introduced into the assay kit in the presence or absence of molecules which may sequester the microcystin LR and reduce the observed sıgnal: For example, a test of this system with the microcystin LR antibody produced an apparent huge decrease in the sensitivity of the kit, shifting the binding isotherm to higher microcystin concentrations (Figure 5.7). When this experiment was repeated with the DNA sequences the results were much less dramatic, however the sigmoidal shape of the curves indicated that DNA sequences did bind to microcystin LR (Figures 5.8-5.10). The shift in the binding isotherm to a sigmoidal shape which had to be modeled using the Hill equation indicated the each of the sequences were able to interfere with the binding of the microcystin LR to the antibodies in the test kit. However, potentially due to larger $K_{\text {off }}$ binding components associated with antibodies and the clustering of the antibodies in the reaction wells, which is known to produce increased apparent binding affinity values (Caré and Soula, 2011), the slopes of the curves in the presence of the aptamer sequences deviated from the binding isotherm associated with mass action effects. Sigmoidal binding curves can be modeled using the Hill equation (Hill 1910) and are 


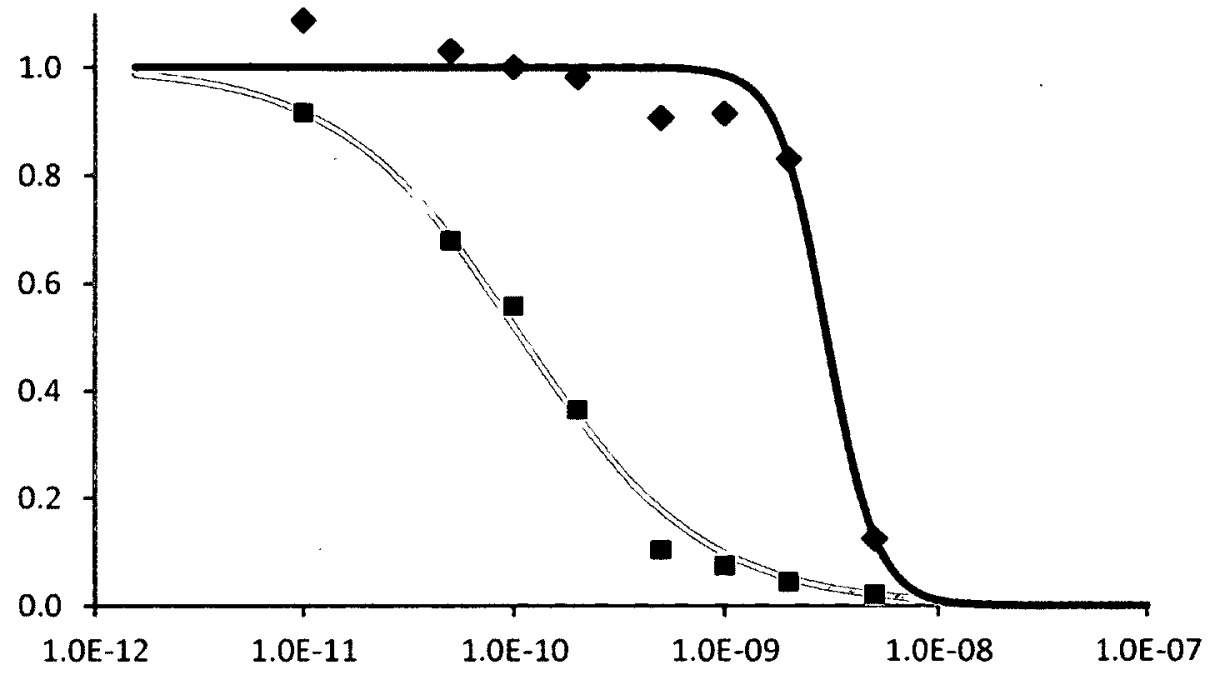

Figure 5.7. ELISA plot of microcystin LR binding, corresponding to a decrease in enzymatic activity as microcystin LR concentrations were increased from $1 \times 10^{-11}$ to $5 \times 10^{-}$

${ }^{9} \mathrm{M}$ (grey line) and the concurrent interference in the binding profile produced by the presence of the free antibody $\left(1 \times 10^{-9} \mathrm{M}\right.$, black line $)$. 


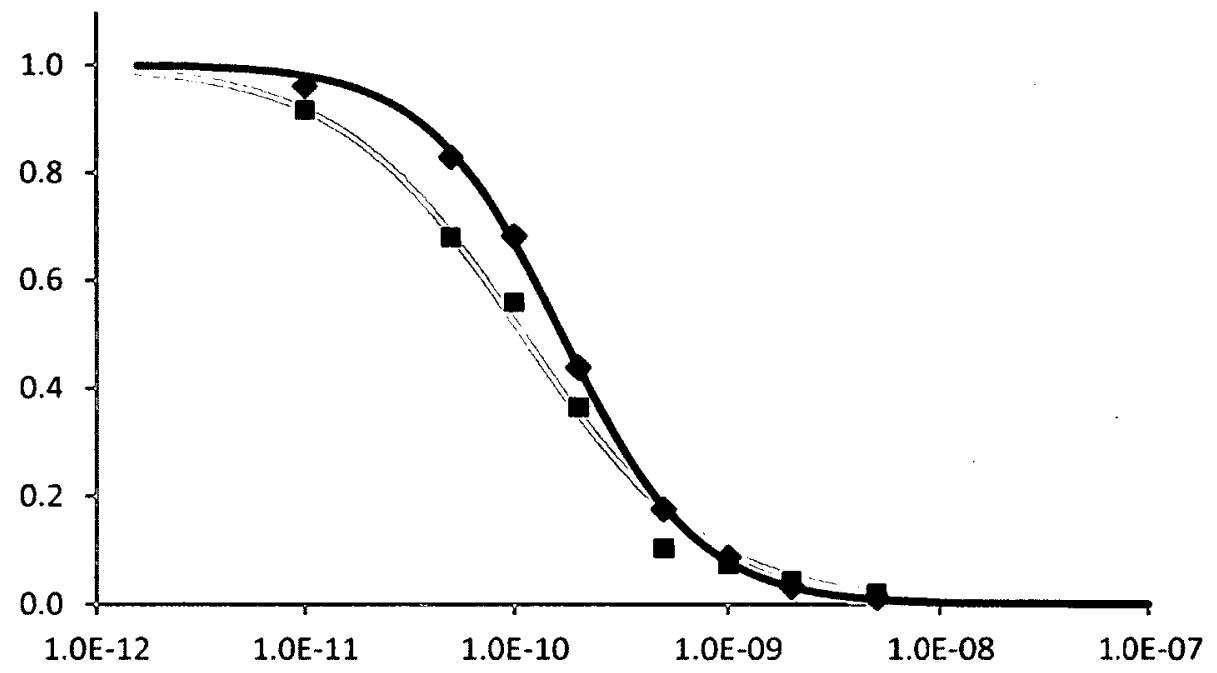

Figure 5.8. ELISA plot of microcystin LR binding, corresponding to a decrease in enzymatic activity as microcystin LR concentrations were increased from $1 \times 10^{-11}$ to $5 \times 10^{-}$ ${ }^{9} \mathrm{M}$ (grey line) and the concurrent interference in the binding profile produced by the presence of the $\mathrm{H} 18$ aptamer $\left(1 \times 10^{-6} \mathrm{M}\right.$, black line $)$. 


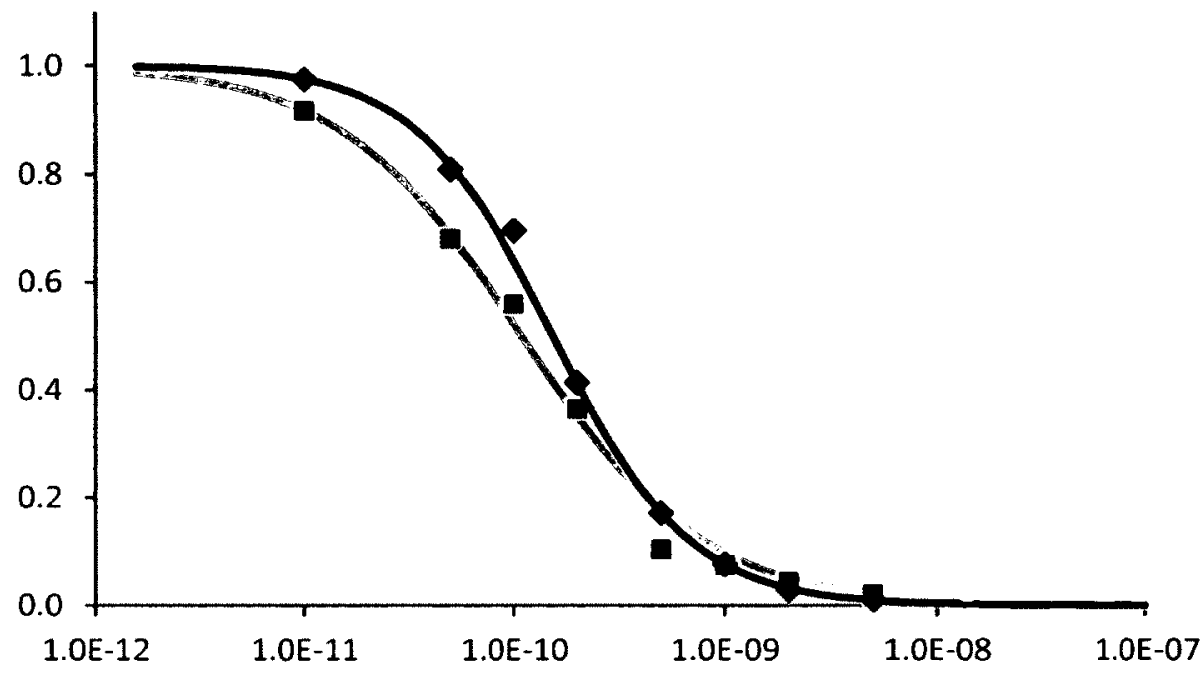

Figure 5.9. ELISA plot of microcystin LR binding, corresponding to a decrease in enzymatic activity as microcystin LR concentrations were increased from $1 \times 10^{-11}$ to $5 \times 10^{-}$ ${ }^{9} \mathrm{M}$ (grey line) and the concurrent interference in the binding profile produced by the presence of the $\mathrm{H} 12$ aptamer $\left(1 \times 10^{-6} \mathrm{M}\right.$, black line $)$. 


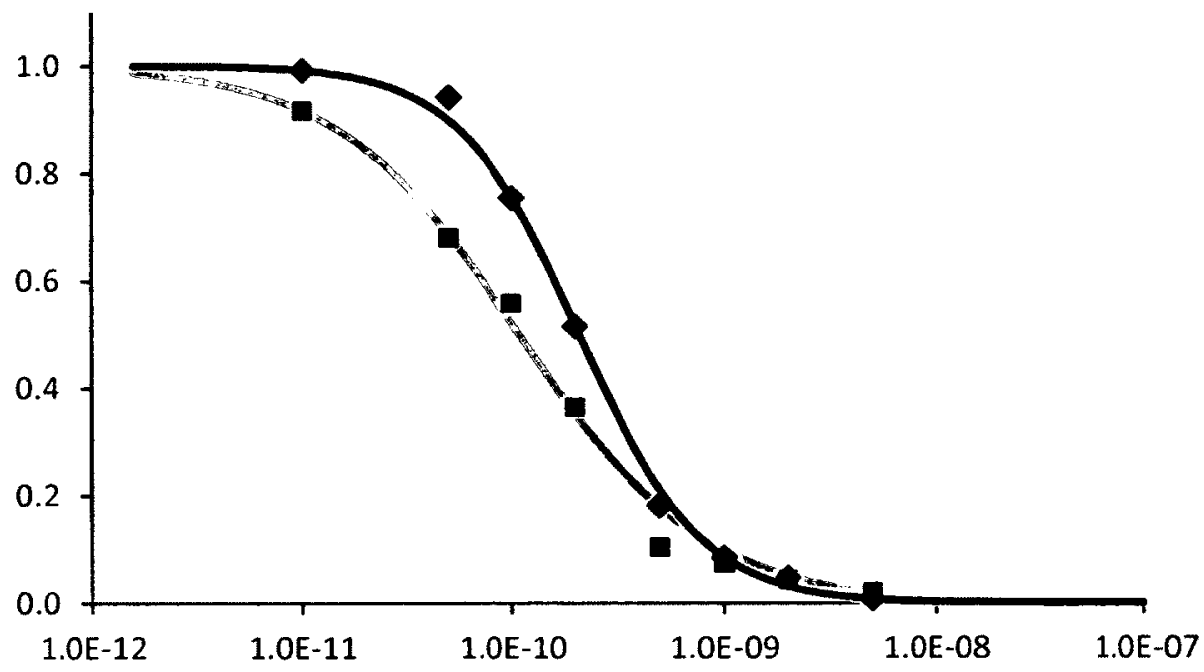

Figure 5.10. ELISA plot of microcystin LR binding, corresponding to a decrease in enzymatic activity as microcystin $L R$ concentrations were increased from $1 \times 10^{-11}$ to $5 \times 10^{-}$ ${ }^{9} \mathrm{M}$ (grey line) and the concurrent interference in the binding profile produced by the presence of the LRL 3 aptamer $\left(1 \times 10^{-6} \mathrm{M}\right.$, black line $)$. 
usually equated to cooperative binding, but when associated with competitive binding assays limit the amount of useful data that can be obtained (Motulsky and Christopoulos, 2004). Therefore, while indicating that there was binding between the aptamers and microcystin LR this study was not able to provide any additional quantitative information on the affinity of the aptamers for microcystin LR.

While the preceding studies were unable to definitively characterize the binding affinity between the aptamers and microcystin LR, further confidential work using surface plasmon resonance of surface bound microcystin suggests that the binding affinity of the $\mathrm{H} 12$ aptamer in their system was close to $1.5 \mu \mathrm{M}$ (Figure 5.11).

While the selection of aptamers for microcystin LR was fairly straight forward, the characterization of their binding affinity presented similar challenges to those encountered in chapter 2 with the additional complexity that microcystin LR was not fluorescent. This unfortunately prevented the complete characterization of the binding constants for the aptamers produced here, however :t: did highlight the difference in binding interactions between most aptamer systems and antibodies. The high affinity of antibodies for their targets might be equated to low $K_{\text {off }}$ values where the light and heavy chain can act almost like a chelation system and lock their target between them. The low dissociation of the microcystin from the antibody is quite apparent in Figure 5.7. In contrast, the high on off rate associated with the aptamer sequences generated in this selection is highlighted by the curves produced when they were incubated with microcystin LR in the wells containing the antibodies. In the 30 minute equilibration 


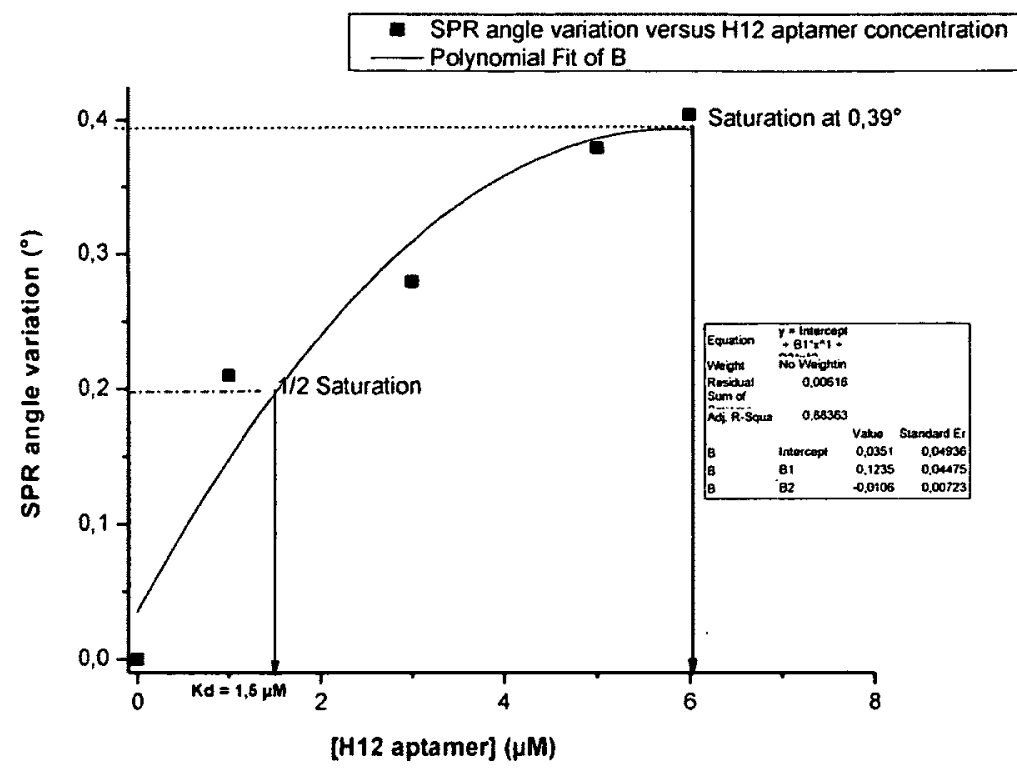

Figure 5.11. SPR angle variation versus $H 12$ aptamer concentration resulting in a dissociation constant of $1.5 \mu \mathrm{M}$. 
period the antibody was almost able to bind as much microcystin LR as was observed in the control without aptamers present. This suggests a simple way of improving the $K_{\text {off }}$ binding component of aptamers though secondary selection processes where instead of starting with a completely random pool, selection could be initiated with a random sequence attached to a known aptamer. This would potentially provide almost single round improvement to the binding characteristics, for certain aptamer sequences, by providing an additional subunit to the aptamer sequence allowing for enhanced target binding through reduction of $K_{\text {off }}$ rates.

Further evaluation of the aptamer sequences produced in this project is ongoing. While the affinity of the aptamers produced in this study may be less than that of the antibody for microcystin LR, as demonstrated by the competitive binding assays, the functionality of DNA as a nanomaterial suggest several ways of dealing with sensitivity issues. In addition to the proposed selection for improving $K_{\text {off }}$ values detailed above, the ease with which aptamers can be produced allows for improved detection through receptor clustering (Caré and Soula, 2011) which may be able to push detection limits for microcystin LR well into the subnanomolar range making technologies based on aptamer detection relevant for drinking-water quality assessment. 


\section{Acknowledgements}

I would like to thank our collaborator Dr Rocio Aranda-Rodriguez and her postdoctoral fellow Dr Hechun Liu for his work on the aptamer selection portion of this study. I would also like to thank Dr Rocio Aranda-Rodriguez's other postdoctoral fellow Dr. Alexander Wahba for his involvement in the project and work on the ELISA competitive binding experiments.

I would also like to thank Dr. Tito Scaiano and his postdoctoral fellows Dr. Pacioni Natalia and Dr. Alarcón Abarzúa Emilio Isaac, Department of Chemistry Ottawa University, for their hospitality and assistance with the Circular dichroism portion of this study. 
Table 5.1. Microcystin LR binding affinities determined using anisotropy and circular dichroism for sequences generated from the microcystin LR SELEX.

\begin{tabular}{|l|l|l|l|}
\hline & $\mathrm{H} 12$ & $\mathrm{H} 18$ & LRL3 \\
\hline Anisotropy & $9 \pm 4 \mathrm{nM}$ & - & - \\
\hline Circular dichroism & $58 \pm 13 \mathrm{nM}$ & $67 \pm 26 \mathrm{nM}$ & ambiguous \\
\hline Surface Plasmon Resonance & $1.5 \mu \mathrm{M}$ & & \\
\hline
\end{tabular}


Chapter 6: Systematic Evolution of Ligands by EXponential enrichment (SELEX) for DNA aptamers which modulate the catalytic activity of Kallikrein 


\begin{abstract}
Plasma kallikrein is a trypsin-like protease, an enzyme class known to be subject to allosteric modulation. Trypsin-like proteases are a large family of enzymes involved in many physiological processes. Plasma kallikrein is primarily involved in coagulation, inflammation and the regulation of blood pressure and its dysregulation has been associated with neurodegenerative diseases and implicated in diabetic macular edema. Here we describe an aptamer selection procedure using kallikrein to illustrate a way of discovering sequences which can modulate the activity of trypsin-like proteases. By collecting the pool which elutes from the selection chamber by fractionation the pool separates itself based on kallikrein affinity and the eluted fractions can be assessed for their ability to modulate enzymatic activity of kallikrein. Preliminary analysis of the selection fractions suggests that this methodology may be able to generate sequences which are able to stimulate or to inhibit the activity kallikrein. This suggests that this selection methodology may offer a novel way of probing the activity of trypsin-like proteases and producing tools for both assessing their role in diseases and modulating their activity for therapeutic purpose.
\end{abstract}




\section{Introduction}

The intrinsic selectivity and high affinity binding characteristics of aptamers has resulted in a rush to exploit these molecules in the development of systems for the targeted delivery of therapeutics (Tan et al., 2011). Aptamers are specifically attractive for physiological applications due to their low degree of immunogenicity and high degree of biological compatibility (Soontornworajit and Wang, 2011). These properties also make aptamers ideal candidates as therapeutics themselves (Bunka et al., 2010). Aptamers are beginning to be used to target diverse enzymatic activities with some reaching the therapeutic trial stage, such as thrombin inhibitors for modulation of the coagulation cascade (Mayer et al., 2011). The adaptability of aptamers has also allowed for their inclusion in increasingly sophisticated therapeutic strategies, such as the inhibition of enzymes found in tumors that reduce the efficacy of chemotherapies (Krylova et al., 2012).

As most therapeutics are known to affect the catalytic activity of enzymes (Walsh et al., 2011) and with the therapeutic potential of aptamers to act as enzyme inhibitors, a selection project was undertaken to investigate the ability of DNA to modulate the catalytic activity of the enzyme plasma kallikrein [E.C. 3.4.21.34] (Figure 6.1 and 6.2). Plasma kallikrein is a protease a class of enzyme which are receiving a fair bit of interest as targets for therapeutic aptamer development (Dupont et al., 2011). Plasma kallikrein is involved in coagulation and the regulation of blood pressure (Bryant and ShariatMadar, 2009). Dysfunction of the kallikrein system has been implicated in neurological disorders such as Alzheimer's disease and vascular dementia (Ashby et al., 2010). It has 
also been linked to the symptomatic retinal vascular pathology associated with diabetes (Clermont et al., 2011).

Plasma kallikrein is a part of the $\$ 1$ peptidase family, which is the largest peptidase family, characterized by their serine endopeptidases activity (Page and Cera, 2008). A common catalytic triad composed of a serine, aspartate and histidine at the active site (Figure 6.1) and their catalytic specificity is reliant on the recognition of amide bonds following the P1 position. In the case of kallikrein the S1 binding site accommodates the side chain of lysine or arginine allowing it to cleave the following amide peptide bond. The enzymatic activities of trypsin-like proteases are highly regulated as these enzymes are initially produced as zymogens which have to be proteolytically activated to the mature catalytically active form (Gohara and Di Cera 2011). Additionally, mounting evidence suggests that all of these enzymes are allosterically regulated causing them to switch between an open active form and a collapsed form which inhibits their activity (Gohara and Di Cera 2011).

The study described here is still ongoing, however, initial results suggest that the selection pool contains sequences which inhibit and sequences which activate the activity of plasma kallikrein. While inhibition is quite common, enzymatic activators are quite rare (Walsh, 2012) and the potential of producing both provides a unique opportunity for probing the kinetic and physiological properties of this enzyme (Gohara and Di Cera 2011). 


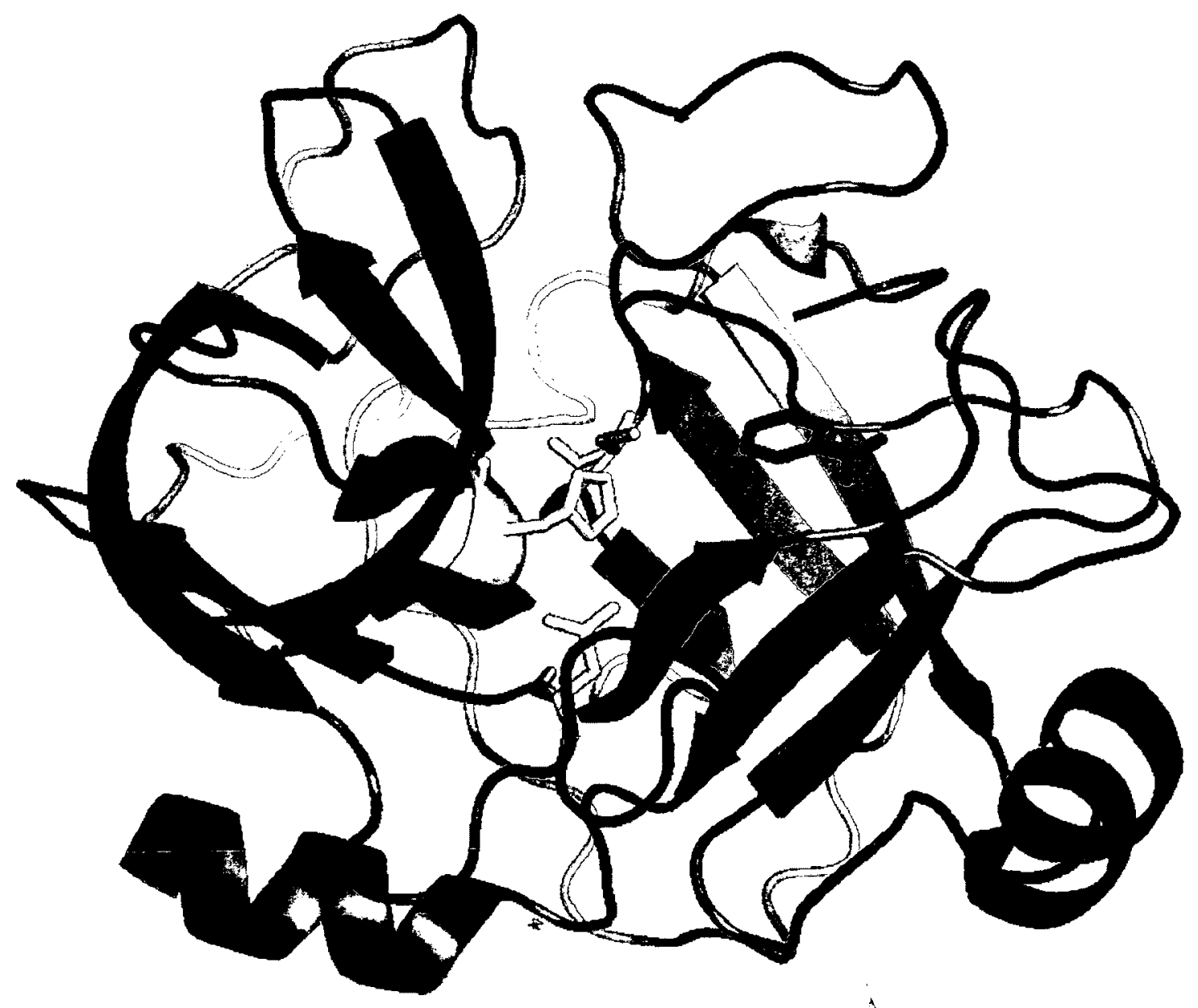

Figure 6.1. Human kallikrein 6 with the catalytic triad of the active site highlighted in yellow and the inhibitor benzamidine (red) which is known to bind the S1 pocket of the enzyme. This figure was generated from the using pymol (Delano 2002) and the crystal structure deposited by Bernett et al., (2002) in the protein data base (PDB ID: 1L2E). 


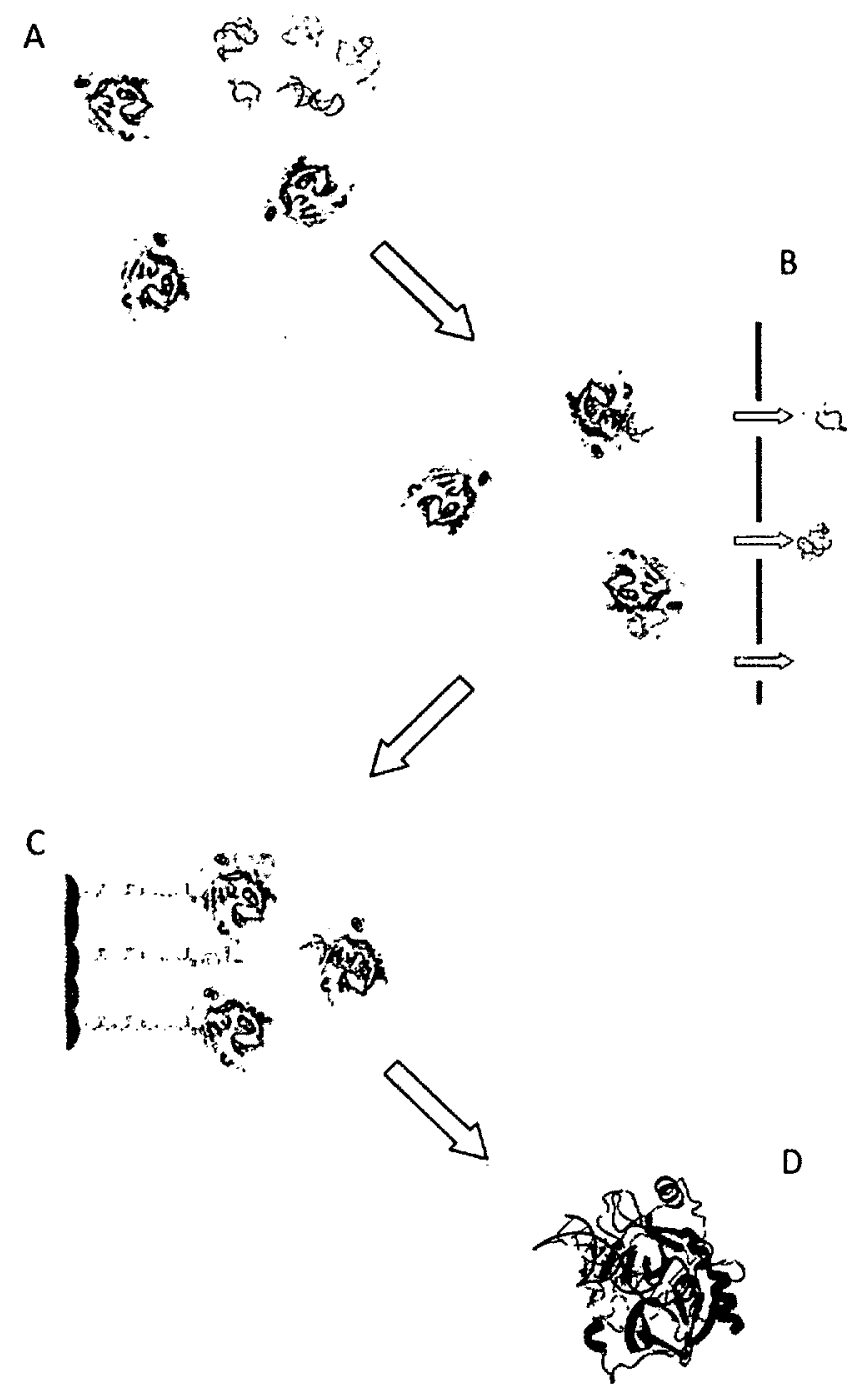

Figure 6.2. Schematic for the selection of aptamers which inhibit kallikrein activity involving a four step procedure. A) Initially the DNA pool was mixed with kallikrein followed by B) washing away of unbound sequences through a filter which prevented loss of the enzyme. C) The enzyme sample was then exposed to competitive selection on a benzamidine column to separate sequences which bound the active site of the enzyme from those that bound other sites of the enzyme. D) Isolated sequences were amplified with PCR and analysis for activity modulation 


\section{Materials and methods:}

Bovine serum albumin (BSA) and Human plasma kallikrein ([E.C. 3.4.21.34], $M W=85000$ Da) were purchased from Sigma-Aldrich. HiTrap ${ }^{\mathrm{TM}}$ Benzamidine affinity column material was acquired from GE Healthcare. The Kallikrein substrate analogue D-Val-Leu-Arg-pNA was purchased from PolyPeptide Lab San Diego. Ultrafiltration Membranes NMWL 30,000 and 50,000 were purchased from EMD Millipore.

Primer 1: 5'-ATACCAGCTTATT-3'

Primer 2: 5'-AGATTGCACTTACTATCT-3'

Primer $\mathbf{2}$ with Poly $\mathrm{A}_{20}$ tail and ethyleneglycol spacer:

5'-AAAAAAAAAAAAAAAAAAAAXAGATTGCACTTACTATCT-3'

DNA Pool sequence ( $M W=15499 \mathrm{Da})$

$5^{\prime}-$

ATACCAGCTTATTCAATTGCNNNNGCAATTNNNGTCNGGACNNNNGTTCNGACNNTCGGCGN NNCGCCGANCTATCTNNNNNAGATAGTAAGTGCAATCT-3'

The above sequences were proposed for aptamer selection by Luo et al., (2010) where $\mathbf{R}$ represents the Fluorescein label 6-Fluorescein Phosphoramidite, $\mathbf{X}$ represents Spacer Phosphoramidite 18 the hexaethyleneglycol spacer which prevents TAQ 
polymerase read through and $\mathbf{N}$ represents an indeterminate random base in the sequence which could be adenine, thymine, cytosine or guanine.

\section{Selection for Kallikrein activity modifying aptamers}

Aptamer selection was performed using a selection buffer based on the buffer used to assess the activity of kallikrein (Geiger, 1984), $100 \mathrm{mM}$ Tris $\mathrm{HCl} \mathrm{pH} \mathrm{8.2,0.05} \mathrm{mM}$ EDTA. The DNA pool used for selection was initially heated to $90^{\circ} \mathrm{C}$ for 5 minutes followed by cooling to room temperature. The pool was then added to a $10 \mathrm{~mL}$ Millipore Amicon stirred cell and filtered through a Ultrafiltration Membrane $(50,000$ NMWL) under 5 psi argon. Each Ultrafiltration membrane was prewashed with two 5 $\mathrm{mL}$ volumes of $\mathrm{dH}_{2} \mathrm{O}$ and pre-equilibrated by passing $10-15 \mathrm{mLs}$ of selection buffer through the stirred cell prior to the selection. The filtered pool from the first stirred cell was then added to another stirred cell containing the target enzyme Kallikrein $(\sim 0.05$ $\mathrm{mU}$ ) suspended in $1 \mathrm{~mL}$ of selection buffer. The sample volume was adjusted to $10 \mathrm{mLs}$ with selection buffer and then left to stir for 10 minutes. Argon pressure was then adjusted to $5 \mathrm{psi}$ and used to remove $9 \mathrm{mLs}$ of the sample. The removed buffer was replaced with an additional $9 \mathrm{mLs}$ of selection buffer. This process was repeated 16 times to produce a successive logarithmic dilution of the DNA pool. The remaining approximate $1 \mathrm{~mL}$ sample in the stirred cell was combined with $5 \mathrm{mg}$ of benzamidine affinity column in a micro-spin filter tube $(0.45 \mu \mathrm{m}$ nylon filters $)$. The column material had been pre-equilibrated with $1.5 \mathrm{mLs}$ of selection buffer. The enzyme and 
benzamidine affinity column were incubated at room temperature on a shaken for 10 minutes before being centrifuged at $3000 \mathrm{~g}$ for 1 minute. The DNA sample recovered from the benzamidine column was dried down to prepare it for PCR and isolation (See Below).

PCR was unable to amplify any sequences associated with the kallikrein sample subjected to secondary selection on the benzamidine affinity column. PCR amplification was then used to sequentially examine the 16 elution samples starting with the $16^{\text {th }}$ and working back. No amplification was observed with elutions $12 ; 16$. When elutions 7-11 were examined amplification was observed in elution 7, and the corresponding gel region for elutions 8-11 was also isolated. Fraction 7 and fractions 8-11 were PCR amplified separately and isolated for the second round of selection.

The second round of selection followed the same procedure as the first with the exception that the elution pattern observed in the first round was used as a guide to determine the number of buffer washes which would be required to get the two amplified pools to a concentration comparable to that observed in the $7^{\text {th }}$ fraction of the first round of selection. The enzyme samples recovered from the benzamidine column were dried down and amplified using PCR before isolation.

Round three of the selection was performed on the sample generated from fraction 7 of round two. While the selection process was similar to the selection used in round 2 there were a few alterations. Round three for fraction 7 involved the introduction of $0.1 \% \mathrm{BSA}(\mathrm{W} / \mathrm{V})$ to the selection buffer. This round also omitted the guard filter so that 
the pool and kallikrein were mixed and then added to the stirred cell. Five $9 \mathrm{~mL}$ fractions were collected by adding selection buffer without the BSA to the stirred cell and the final approximate $1 \mathrm{~mL}$ was collected for addition to a $1 \mathrm{mg}$ benzamidine column before elution and preparation for the next round of selection.

In round four of the selection, the DNA pool, generated from fraction 7 of the first round, was once again subjected to a pre-filtering step. The pool sample used in this round was partitioned into an approximate equivalent number of moles as number of moles of kallikrein to be used in the selection $\left(\sim 0.4 \times 10^{-12} \mathrm{~mol}\right.$ pool to $2 \times 10^{-12} \mathrm{~mol}$ kallikrein). The $1 \mathrm{~mL}$ sample was filtered through an Ultrafiltration Membrane $(50,000$ NMWL) with five $1 \mathrm{~mL}$ volumes of selection buffer to ensure full transfer to the second stirred cell. The second stirred cell containing the target enzyme Kallikrein suspended in $1 \mathrm{~mL}$ of selection buffer. The sample volume was adjusted to $10 \mathrm{mLs}$ with $1 \% \mathrm{BSA}$ selection buffer before the cell was pressurized with argon ( 5 psi) which was used to remove $91 \mathrm{~mL}$ fractions of the sample. The removed buffer was replaced with an additional $9 \mathrm{mLs}$ of selection buffer which was then collected as a $9 \mathrm{~mL}$ fraction. This process was repeated 4 more times and then the $1 \mathrm{~mL}$ fraction remaining in the stirred cell was also collected. All of the fractions were dried down for PCR amplification and isolation.

The pool sample generated from fraction 8-11 of round one were subjected to a third round of selection. During this round of selection the same procedure used in the $4^{\text {th }}$ selection round for the pool sample generated from fraction 7 were applied (see 
preceding paragraph), except that the 50,000 NMWL Ultrafiltration Membrane was replaced with a 30,000 NMWL Ultrafiltration Membrane due to a discontinuation of the 50,000 NMWL Membranes.

\section{PCR}

Samples to be amplified were suspended in reaction buffer consisting of $100 \mathrm{mM}$ Tris $\mathrm{HCl} \mathrm{pH} \mathrm{9,} 50 \mathrm{mM} \mathrm{KCl}, 1 \%$ Triton X-100, $2 \mathrm{mM} \mathrm{MgCl} 2,0.5 \mathrm{mM}$ dNTPs, $1 \mu \mathrm{M}$ of each primer and $5 \mathrm{U}$ of Taq DNA polymerase in a final volume $100 \mu \mathrm{L}$. Samples were amplified using an Eppendorf Mastercycler EP Gradient, Thermal Cycler programmed to initially denature samples at $95^{\circ} \mathrm{C}$ for 2 minutes followed by 30 rounds of amplification. Amplification cycles consisted of primer annealing at $57^{\circ} \mathrm{C}$ for 1 minute, followed by 1 minute of template amplification $72^{\circ} \mathrm{C}$ and concluding with sample denaturation for 30 seconds at $95^{\circ} \mathrm{C}$ before initiation of the following amplification cycle. The amplification process concluded with an extended 5 minute $72^{\circ} \mathrm{C}$ template amplification phase before samples were cooled to $4^{\circ} \mathrm{C}$ before processing. Amplified samples were dried down using a Savant DNA120 SpeedVac Concentrator, Thermo Electron Corporation, in preparation for electrophoretic isolation. 


\section{Polyacrylamide Gel Electrophoresis}

Samples were isolated with $12 \%$ acrylamide gels cast using $18 \times 16 \mathrm{~cm}$ gel plates. Twelve percent acrylamide gels were cast by combining $31.5 \mathrm{~g}$ of urea, $23.5 \mathrm{mLs}$ of 5.3 $\mathrm{M}$ acrylamide and $130 \mathrm{mM} \mathrm{N}, \mathrm{N}^{\prime}$-methylenebisacrylamide, $15 \mathrm{mLs}$ of $0.44 \mathrm{M}$ Boric acid, $0.44 \mathrm{M}$ Tris, and $10 \mathrm{mM}$ EDTA with $14 \mathrm{mLs} \mathrm{dH}_{2} \mathrm{O}$. The mixture was heated to $37^{\circ} \mathrm{C}$ filtered and allowed to cool to room temperature. This was followed by the addition of $0.45 \mathrm{mLs}$ of $10 \%$ ammonium persulfate followed by $35 \mu \mathrm{L} \quad \mathrm{N}, \mathrm{N}^{\prime} \mathrm{N}^{\prime}, \mathrm{N}^{\prime}-$ tetramethylethylenediamine. The gels were poured and allowed to polymerize for 20 minutes. After polymerization, the gels were set up in a Hoefer SE 600 series vertical slab gel electrophoresis unit and the buffer reservoirs were filled with $88 \mathrm{mM}$ Boric acid, $88 \mathrm{mM}$ Tris, and $2 \mathrm{mM}$ EDTA. Gels were pre-equilibrated typically using a constant current of 35 milliamps for 20 mins using a FisherBiotech Power Suppy FB1000. DNA samples were dissolved in minimal amounts of $\mathrm{dH}_{2} \mathrm{O}$ and mixed with an equal amount of formamide before being heated at $55^{\circ} \mathrm{C}$ for 5 minutes. This was followed by loading onto the gel for separation. The gels were subjected typically to a constant current of 35 milliamps for 2 hours.

Upon completion of the electrophoretic run the gels were imaged using an Alpha Innotech imager. Bands of interest were cut out and the gel crushed and suspended in 5 times the volume of $10 \mathrm{mM}$ Tris $\mathrm{pH}$ 7.4. Samples were frozen using liquid nitrogen followed by heating to $90^{\circ} \mathrm{C}$ for 10 minutes. The samples were then allowed to mix and incubated overnight at $37^{\circ} \mathrm{C}$. Following incubation, the samples were filtered $(0.45 \mu \mathrm{m})$ to remove the remaining gel fragments. Samples were then lyophilized to reduce the 
sample volume followed by resuspension in a minimal volume of deionized water for desalting and concentration. Samples were concentrated initially followed by 4 rounds of deionization with deionized water using 3,000 NMWL Millipore Microcon centrifugal filter devices $12000 \mathrm{~g}$ for 30 minutes. Samples were then quantified by assessing their absorbance at $260 \mathrm{~nm}$ and lyophilized.

\section{Kallikrein activity studies}

Enzyme kinetic studies were preformed according to Geiger (1984). Enzymatic activity was examined in $100 \mathrm{mM}$ Tris $\mathrm{HCl} \mathrm{pH} 8.2,0.05 \mathrm{mM}$ EDTA assay buffer. Kallikrein, the substrate D-Val-Leu-Arg-pNA and the DNA samples which were tested for modulatory activity were all suspended in assay buffer prior to initiation of the reactions. DNA samples were heated to $90^{\circ} \mathrm{C}$ for 5 minutes and cooled to room temperature prior to the assay. Reactions were typically preformed by adding $5 \mu \mathrm{L}$ of enzyme, which had been premixed to approximately $1 \mathrm{mU} / \mathrm{mL}$ (approximate concentration $2 \times 10^{-9} \mathrm{M}$ ), and $5 \mu \mathrm{L}$ of DNA to $85 \mu \mathrm{L}$ of reaction buffer, or $90 \mu \mathrm{L}$ of buffer when DNA was excluded, to produce a final volume of $95 \mu \mathrm{L}$. Reaction mixtures were kept on ice before being transferred to a quartz cuvette. Reactions were initiated with the addition of $5 \mu \mathrm{L} 0.2 \mathrm{mM}$ substrate. Substrate hydrolysis was monitored at $405 \mathrm{~nm}$ through the detection of $p$-nitroaniline release $\left(\varepsilon=10210 \mathrm{M}^{-1} \mathrm{~cm}^{-1}\right.$; Geiger, 1984). 


\section{Results and Discussion}

The pool sequence used in this selection was proposed by Luo et al., (2010), and included twenty four random nucleotides, representing approximately $3 \times 10^{14}$ potential sequences. The positioning of the random portions of the sequence was generated to produce an increased distribution of complex secondary structures within the pool. Most aptamers which have been discovered are simple stem-loops structures, so the pool design was specifically produced to introduce a higher frequency of junctions based on the notion higher structural complexity may produce better aptamer candidates. The initial pool consisted of approximately 52 nmoles $\left(3 \times 10^{16}\right.$ sequences) maximizing the sequence space covered by the randomly generated pool.

Initially, the selection methodology for this line of experiments was designed to focus on the isolation of potential inhibitors of plasma kallikrein (Figure 6.2). The elution steps in selection round one were designed to wash away all but the sequences with the highest affinity for the enzyme. This was to be followed by a competitive interaction with the benzamidine column, a known inhibitor of the kallikrein enzyme (Figure 6.1; Bernett et al., 2002). It was postulated that enzymes which were bound by aptamers which blocked the active site would also block the binding of benzamidine and flow through the column. Alternatively, aptamers displaced by binding of the kallikrein to the benzamidine column would also potentially possess some inhibitory activity. The binding of the enzyme to the column would also serve a role in sequestering aptamer sequences which bound to sites other than the active site of kallikrein. 
The initial elution profile was designed based on the original concentration of the pool such that the serial dilution would result is several hundred DNA sequences being retained prior to addition of the kallikrein sample to the benzamidine column. However the sample recovered from the benzamidine column did not amplify with PCR. Therefore, fractions which were eluted from the stirred cell were subjected to PCR to isolate the sequences with the most affinity for kallikrein. Working backwards from the last elution, fraction 7 was observed to amplify with PCR so was isolated. Additionally, the other fractions run with fraction 7 , which did not produce amplification, bands 8 through 11 were cut and combined for reamplification with PCK. The reamplification of both samples produced enough DNA to examine potential activity modulation by the fractions. Examination of the fractions suggested potential inhibition associated with the sample derived from fraction 7 and activation associated with the sample derived from fractions 8-11 (Figure 6.3).

The inhibition displayed by fraction 7 was somewhat unusulal in that inhibition was observed to increase up to $20 \%$ with decreasing concentration. This may possibly be rationalized by the diverse mix of sequences present in the pool as observed inhibition is a function of binding affinity, concentration and effect (Walsh 2012). This may mean that the composition of the general pool consists of sequences which when bound exclude the binding of potentially activity modulating sequences at higher concentrations only allowing the detection of activity modulation at lower concentrations. Alternatively, the structure of the pool which was optimized for multi- 

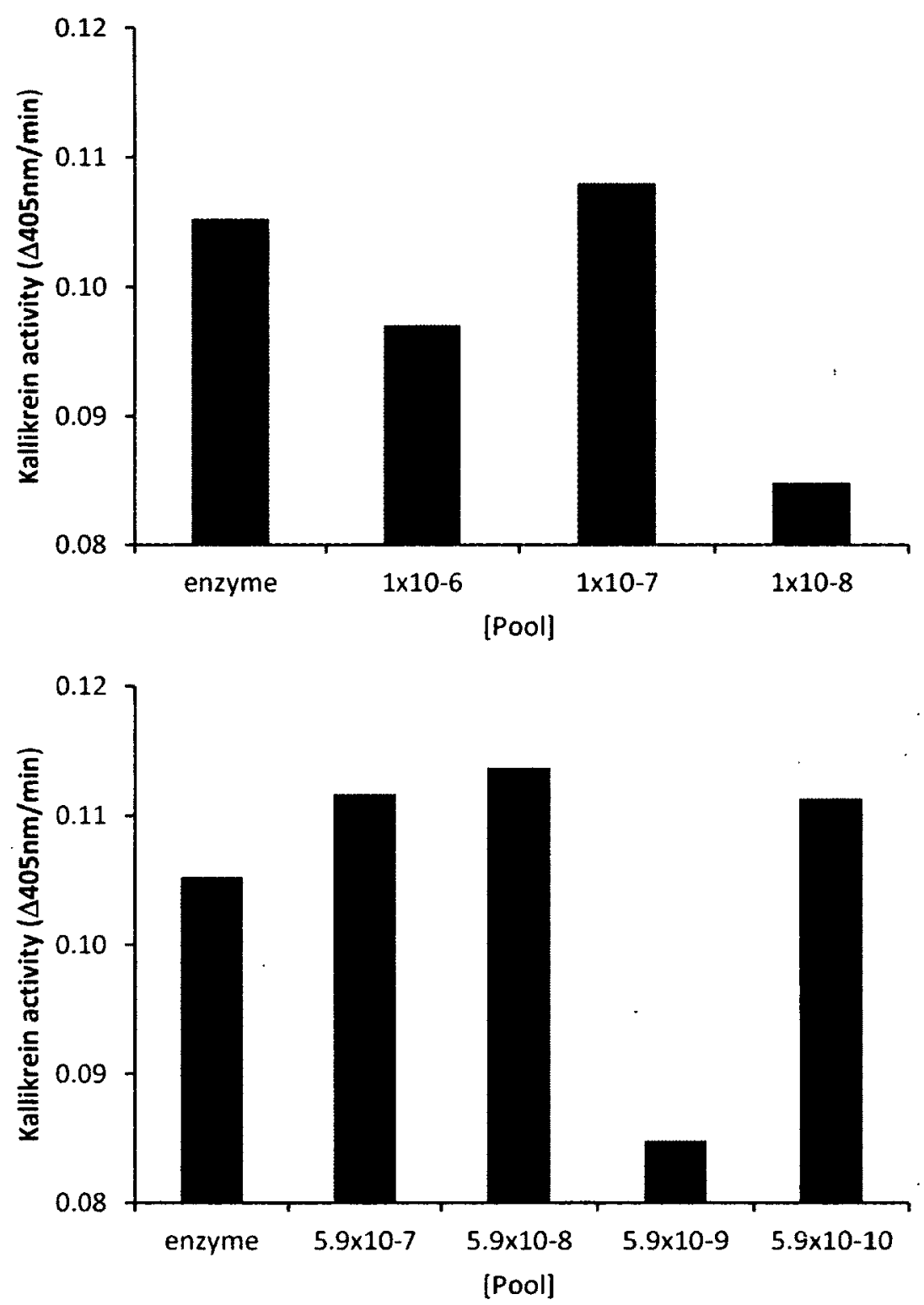

Figure 6.3. Kallikrein activity in the presence of different concentrations of the pools isolated from the first round of selection: A) Sample7 and B) Sample8-11. 
stem formation may promote intermolecular interactions at higher concentrations, which may obscure the modulatory activity.

The activation associated with fractions 8-11 was only about $10 \%$ but this was above the $5 \%$ error associated with the enzyme on its own. Based on this potential activation in the sample isolated from fractions 8-11 (sample8-11) and potential inhibition associated with the sample from fraction 7 (sample7), the decision was made to keep the samples separate for future selection rounds.

Round two of the selection involved the implementation of the full selection process by assuming that the dilution factor that produced fraction seven in the first round represented the lower limit of dilutions that could be performed before the transfer of the kallikrein and pool samples to the benzamidine column. Amplification of samples isolated after the secondary benzamidine column selection in the second round suggested retention of the inhibitory activity associated with sample 7 and the activation associated with sample8-11 (Figure 6.4). The retention of inhibitory activity in sample7 was seen specifically around a dilution of the pool to $2.5 \times 10-7 \mathrm{M}$ which was believed to be attributed to the heterogeneity of sequences making up the sample.

However, concerns over instabilities in the kallikrein activity resulted in the addition of $0.1 \%$ BSA to the selection buffer. BSA has been used to stabilize members of the S1 peptidase family (Kukor et al., 2002) and was observed to improve the stability of plasma kallikrein. 

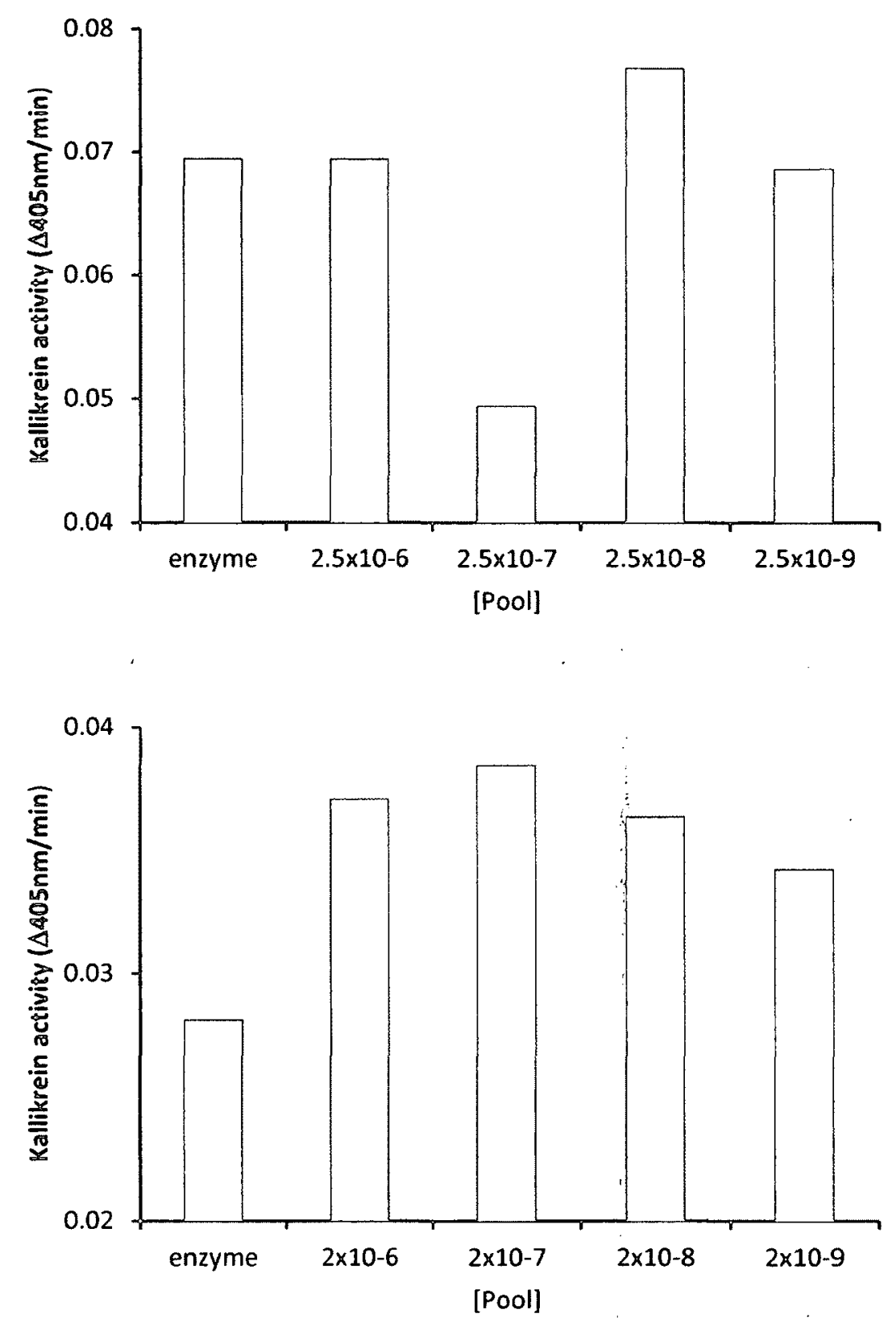

Figure 6.4. Kallikrein activity in the presence of different concentrations of the pools isolated from the second round of selection: A) Sample7 and B) Sample8-11. 


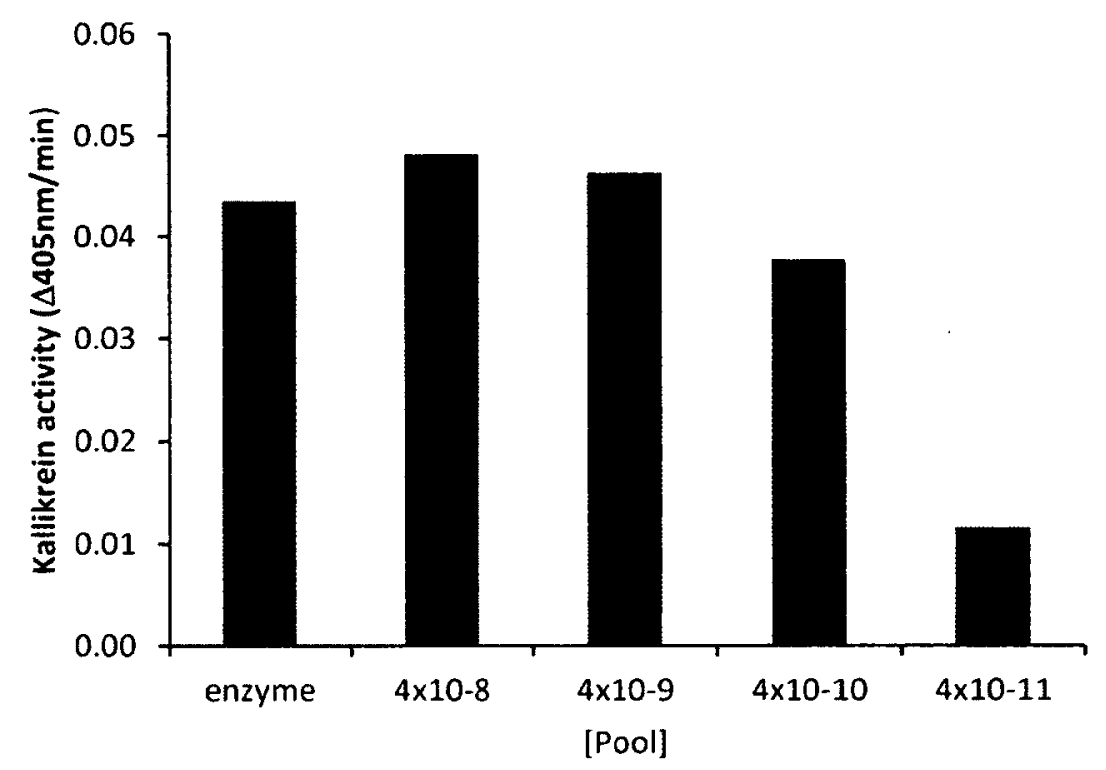

Figure 6.5. Kallikrein activity in the presence of different concentrations of the pools isolated from the third round of selection. Sample derived from fraction 7 of the first round of selection. 
The third selection round performed on sample7 produced similar results to that of round two, in that inhibition was observed to increase with decreasing pool concentration (Figure 6.5).

This sample was re-isolated for a fourth round of selection, however this resulted in a loss of the inhibitory activity (Figure 6.6). A similar loss in activation was observed with the third round of selection with sample8-11 (Figure 6.7). To isolate the inhibitory (sample7) and activation (sample8-11) activities associated with each sample the previous rounds were subjected a modified selection process where the pools were gradually eluted from kallikrein to separate the sequences that were able to modulate the enzymatic activity of kallikrein from those which only possessed binding affinity for the enzyme. Each pool was exposed to the enzyme and gradually eluted in $1 \mathrm{~mL}$ fractions followed by $9 \mathrm{~mL}$ washed resulting in nine $1 \mathrm{~mL}$ fractions and five $9 \mathrm{~mL}$ washed and the $1 \mathrm{~mL}$ remaining in the stirred cell. The fractions were then amplified to assay their activity towards kallikrein. Incubation of the fraction isolated from sample 7 with kallikrein suggested that there were a multitude of activity modulating sequences present in the original sample7 (Figure 6.8). The fiactions were examined at the nanomolar and hundredth of a nanomolar concentration range due to the previously observed inverse inhibitory activity of sample7 and inhibitory activity was observed to eluted in fraction 6 and 7 (Figure 6.8). Reamplification and further characterization shall be assessed in the future. The fractions from sample8-11 have yet to be analyzed. 


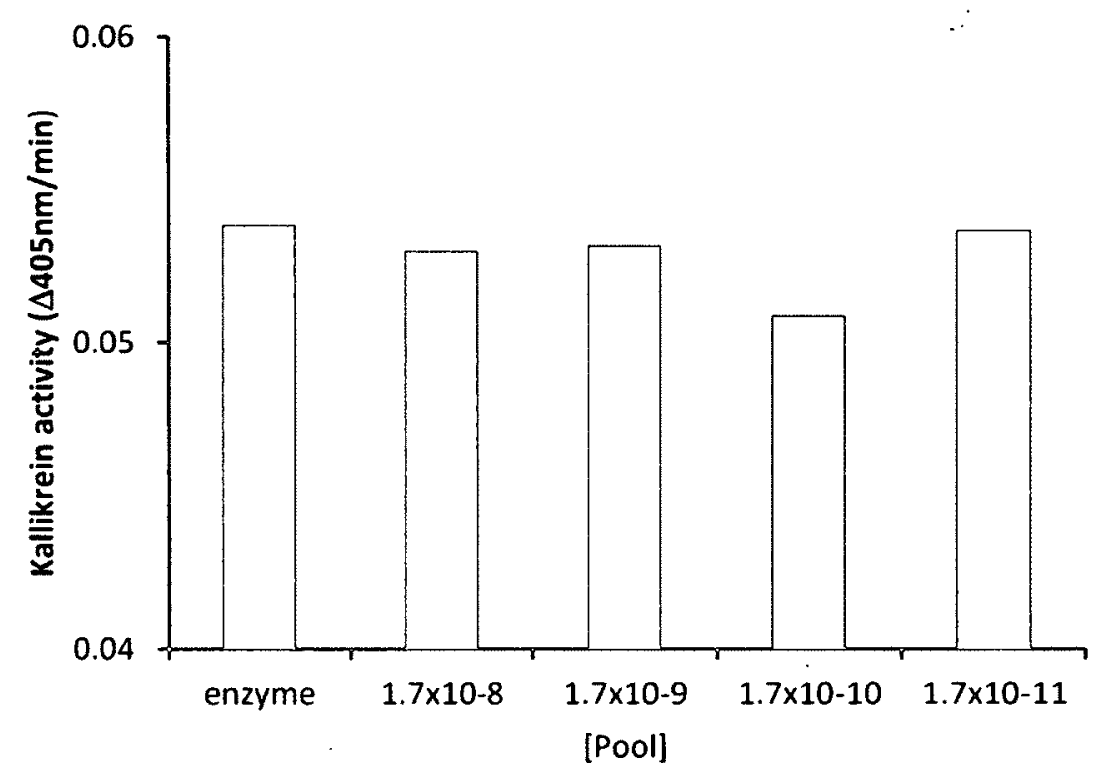

Figure 6.6. Kallikrein activity in the presence of different concentrations of the pools isolated from the fourth round of selection of sample7.

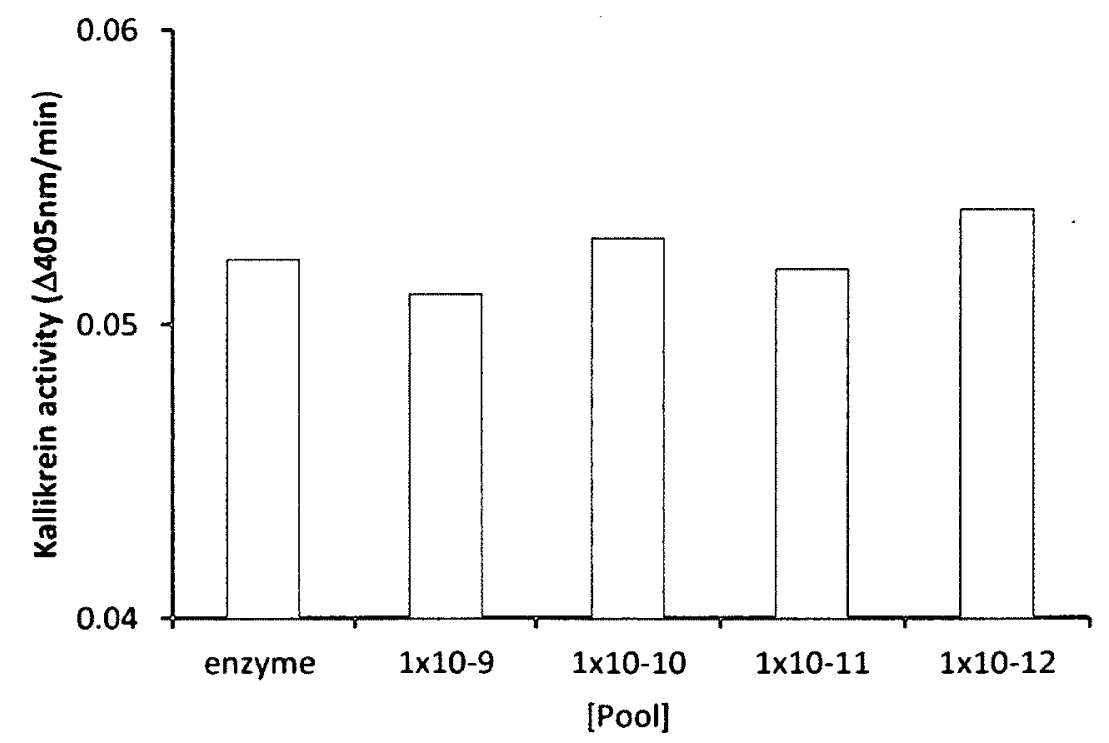

Figure 6.7. Kallikrein activity in the presence of different concentrations of the pools isolated from the third selection round of sample8-11. 
While incomplete, this study suggests that the isolation of DNA aptamers which can modulate the functional activity of peptidases may be a fairly straight forward process. Not only does this method generate inhibitory activity but also surprisingly stimulatory activity as well. The ability to produce stimulatory sequences may be limited to enzymatic systems where the enzyme is subject to regulation of its activity as is the case with kallikrein and members of the S1 peptidase family. However, the ability to isolate sequences which can produce multiple modulatory effects presents a potential invaluable way of probing the functionality of S1 peptidase family and their potential involvement in disease processes (Gohara and Di Cera 2011). This may be of particular interest in the study of Alzheimer's disease where deregulation of S1 peptidases has been suggested as a possible mechanism for the proteolytic production of $\beta$-amyloid from amyloid precursor protein (Darvesh et al., 2001). It may also prove useful in the turning on and off of proteases for therapeutic interventicn in connective tissue diseases (Le Bousse-Kerdilès et al., 2008). 


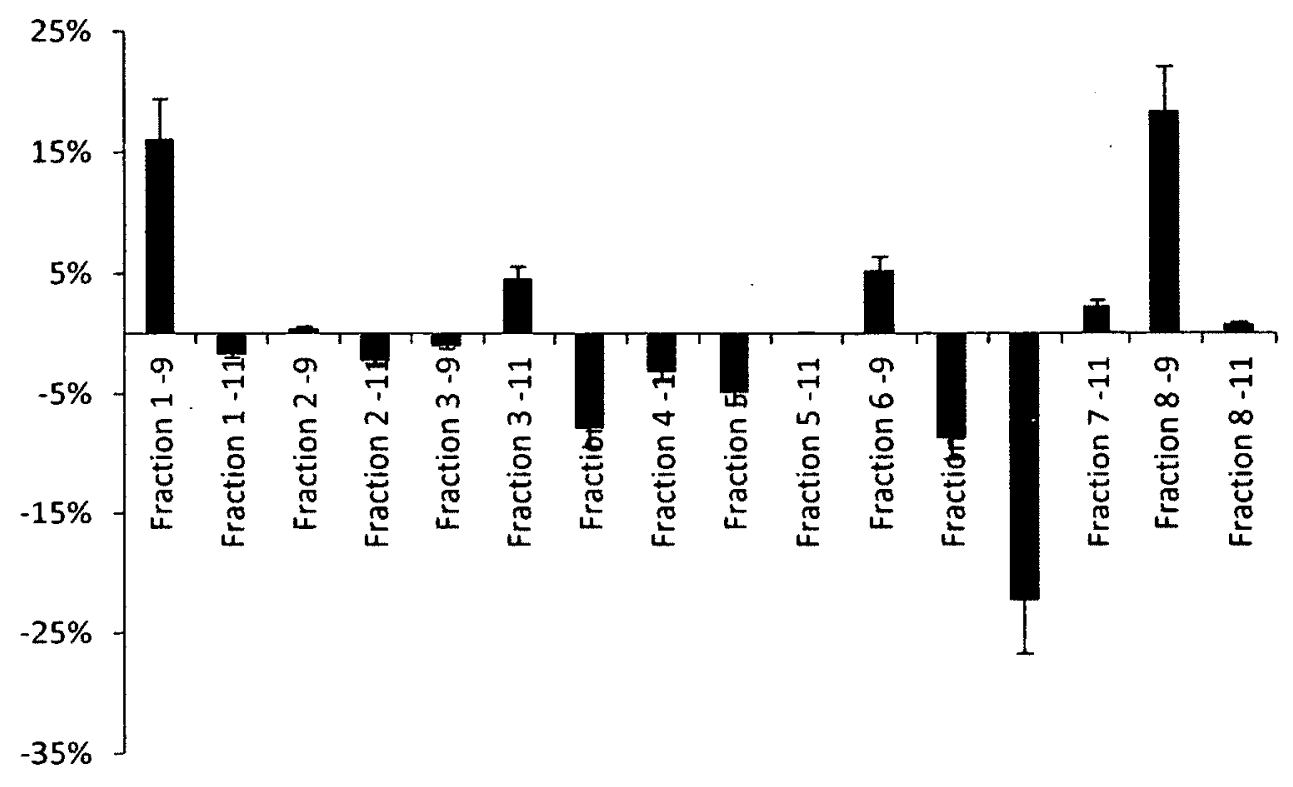

Figure 6.8. Observed change in kallikrein activity associated with elution the first 8 fractions produced from the third round for sample ${ }^{7}$ where fractions were eluted from the enzyme sample. 
While enzymatic inhibitors are quite common there are far fewer examples of activators (Walsh et al., 2011). However, the therapeutic promise of enzymatic activators are being investigated for the treatment of a number of diseases including diabetes (Matschinsky et al., 2011), heart failure (Malik et al., 2011) and elevated cholesterol levels (Ohnsorg et al., 2011). Although activators can be considered a relatively new class of therapeutic the ability to select enzymatic stimulators from pools of random DNA sequences may make them more common as an investigative biological tool and result in increased recognition of therapeutic potentials.

\section{Acknowledgements}

I would like to thank Amirah Yaakob who worked on the initial round of the selection and Xiao Li Wang who continued the project. 


\section{Chapter 7: Overall Conclusions and Future Studies}

The numerous studies described in this thesis demonstrate the utility of DNA as a functional nanomaterial which can equal the structural complexity of RNA and can provide novel selective binding aptamers. The stability of DNA compared to RNA (Wolfenden, 2011) also makes it more suited for biosensor applications and here we used DNA to produce a novel aptamer based selective dopamine biosensor. By coupling the electrochemical signal to selective aptamer binding we were able to produce a dopamine selective sensor reducing the interference resulting from other electrochemically active neurochemicals. Aptamer-based selective electrodes represent a novel way of tailoring electrodes to specific biological systems and should be further investigated. Additional work needs to be done to optimize these dopamine electrodes describe here for in vivo applications.

The ability to produce high affinity aptamers through SELEX represents a revolution in the ability of scientists to study and monitor molecules like microcystin LR and is being utilized extensively at the research level at present. The therapeutic potential of this technology is in its infancy with a few clinical trials underway and even fewer aptamers in clinical use at present.

Aptamers offer a novel route for producing therapeutics as traditional drug development has relied on the use of small molecules where the focus has been on high inhibitory binding constants due to unfavourable side effects. For example tacrine a cholinesterase inhibitor (Darvesh et al., 2003) which treats the symptoms of Alzheimer's 
disease is quite effective also has hepatotoxic side effects which preclude its use at therapeutically relevant levels (Watkins et al., 1994). DNA aptamers have never been reported to be immunogenic or have toxic side effects (Esposito et al., 2011) therefore even without extremely high binding constants aptamers have the potential to be useful as therapeutic agents.

While the use of therapeutic aptamers will definitely increase in the near future our observations with kallikrein may help spur this development. While most therapeutics that modulate the activity of enzymes are inhibitory as they provide simple blockage of the enzymatic active site, our selection was also able to produce activators a much rarer form of enzymatic modulator which may make therapeutics that produce enzymatic activation an attainable drug class.

These studies further demonstrate the utility of DNA as a functional nanomaterial which has the potential to revolutionize bionanotechnologies. 


\section{References}

A. J. Saleh Ahammad, Narayan Chandra Deb Nath, Guang-Ri Xu, Sunghyun Kim, and JaeJoon Lee. (2011) Interference-Free Determination of Dopamine at the Poly(thionine)-Modified Glassy Carbon Electrode. J. Electrochem. Soc. 156:F106F110.

Adams RN. (1976) Probing brain chemistry with electroanalytical techniques. Anal Chem. 48:1126A-1138A.

Agirrezabala X, Frank J. (2010) From DNA to proteins via the ribosome: structural insights into the workings of the translation machinery. Hum Genomics. 4:226-237.

Allongue P, Delamar M, Desbat B, Fagebaume O, Hitmi R, Pinson J, Savéant JM. (1997) Covalent modification of carbon surfaces by aryl radicals generated from the electrochemical reduction of diazonium salts. J. Am. Chem. Soc. 119:201-207.

Amorim A, Vasconcelos V. (1999) Dynamics of microcystins in the mussel Mytilus galloprovincialis. Toxicon 37:1041-1052.

Antao VP, Lai SY, Tinoco I Jr. (1991) A thermodynamic study of unusually stable RNA and DNA hairpins. Nucleic Acids Res. 19:5901-5905.

Ashby EL, Love S, Kehoe PG. (2010) Assessment of activation of the plasma kallikreinkinin system in frontal and temporal cortex in Alzheimer's disease and vascular dementia. Neurobiol Aging. PMID:21074291. 
Baird GS, Nelson SK, Keeney TR, Stewart A, Williams S, Kraemer S, Peskind ER, Montine TJ. (2011) Age-Dependent Changes in the Cerebrospinal Fluid Proteome by Slow Off-Rate Modified Aptamer Array. Am J Pathol.

Baldrich E. (2011) Aptamer arrays. Methods Mol Biol. 671:35-54.

Bannwarth W, Knorr R. (1991) Formation of carboxamides with $N, N, N^{\prime}, N^{\prime}$-tetramethyl (succinimido) uronium tetrafluoroborate in aqueous / organic solvent systems Tetrahedron Lett. 32:1157-1160

Basile VS, Masellis M, Potkin SG, Kennedy JL. (2002) Pharmacogenetics in schizophrenia: the quest for individualized therapy. Hum. Molec. Genet. 11:2517-2530.

Becker B, Cooper MA. (2011) A survey of the 2006-2009 quartz crystal microbalance biosensor literature. J Mol Recognit. 24:754-787.

Berman HM, Westbrook J, Feng Z, Gilliland G, Bhat TN, Weissig H, Shindyalov IN, Bourne PE. (2000) The Protein Data Bank. Nucleic Acids Research 28:235. (http://www.pdb.org/)

Bernett MJ, Blaber SI, Scarisbrick IA, Dhanarajan P, Thompson SM, Blaber M. (2002) Crystal structure and biochemical characterization of humian kallikrein 6 reveals that a trypsin-like kallikrein is expressed in the central nervous system. J Biol Chem. 277:24562-24570. (PDB ID: 1L2E)

Bigott-Hennkens HM, Dannoon S, Lewis MR, Jurisson SS. (2008) In vitro receptor binding assays: general methods and considerations. Q J Nucl Med Mol Imaging. 52:245-253 
Børresen AL. (2002) Mismatch detection using heteroduplex analysis. Curr Protoc Hum Genet. Chapter 7:Unit 7.3.

Boutorine AS, Sun JS. (2005) Postsynthetic functionalization of triple helix-forming oligonucleotides. Methods Mol Biol. 288:251-260.

Brown RV, Hurley LH. (2011) DNA acting like RNA. Biochen Soc Trans. 39:635-640.

Bryant JW, Shariat-Madar Z. (2009) Human plasma kallikrein-kinin system: physiological and biochemical parameters. Cardiovasc Hematol Agents Med Chem. 7:234-250.

Bulyk ML. (2006) DNA microarray technologies for measuring protein-DNA interactions. Curr Opin Biotechnol. 17:422-430.

Bunka DH, Platonova O, Stockley PG. (2010) Development of aptamer therapeutics. Curr Opin Pharmacol. 10:557-562.

Campos A, Vasconcelos V. (2010) Molecular mechanisms of microcystin toxicity in animal cells. Int J Mol Sci. 11:268-287.

Caré BR, Soula HA.(2011) Impact of receptor clustering on ligand binding. BMC Syst Biol. $5: 48$.

Carmon KS, Baltus RE, Luck LA. (2004) A piezoelectric quartz crystal biosensor: the use of two single cysteine mutants of the periplasmic Escherichia coli glucose/galactose receptor as target proteins for the detection of glucosey. Biochemistry 43:1424914256. 
Chartrand P, Harvey SC, Ferbeyre G, Usman N, Cedergren R. (1995) An oligodeoxyribonucleotide that supports catalytic activity in the hammerhead ribozyme domain. Nucleic Acids Res. 23:4092-4096.

Chen T, Shukoor MI, Chen Y, Yuan Q, Zhu Z, Zhao Z, Gulbakan B, Tan W. (2011) Aptamerconjugated nanomaterials for bioanalysis and biotechnology applications. Nanoscale. 3:546-556.

Cho EJ, Lee JW, Ellington AD. (2009) Applications of aptamers as sensors. Annu Rev Anal Chem (Palo Alto Calif). 2:241-264.

Chowrira BM, Berzal-Herranz A, Keller CF, Burke JM. (1993) Four ribose 2'-hydroxyl groups essential for catalytic function of the hairpin libozyme. J Biol Chem. 268:19458-19462.

Clermont A, Chilcote TJ, Kita T, Liu J, Riva P, Sinha S, Feener EP. (2011) Plasma kallikrein mediates retinal vascular dysfunction and induces retinal thickening in diabetic rats. Diabetes. 60:1590-1598.

Colquhoun D. (2006) The quantitative analysis of drug-receptor interactions: a short history. Trends Pharmacol Sci. 27:149-157.

Cooper MA, Singleton VT. (2007) A survey of the 2001 to 2005 quartz crystal microbalance biosensor literature: applications of acoustic physics to the analysis of biomolecular interactions. J Mol Recognit. 20:154-184 
Crawford M, Woodman R, Ko Ferrigno P. (2003) Peptide aptamers: tools for biology and drug discovery. Brief Funct Genomic Proteomic. 2:72-79.

Crespi F, Martin KF, Marsden CA. (1988) Measurement of extracellular basal levels of serotonin in vivo using nafion-coated carbon fibre electrodes combined with differential pulse voltammetry. Neuroscience. 27:885-89€.

Darvesh S, Kumar R, Roberts S, Walsh R, Martin E. (2001) ButyrylcholinesteraseMediated enhancement of the enzymatic activity of trypsin. Cell Mol Neurobiol. 21:285-296.

Darvesh S, Walsh R, Kumar R, Caines A, Roberts S, Magee D, Rockwood K, Martin E. (2003) Inhibition of human cholinesterases by drugs used to treat Alzheimer disease. Alzheimer Dis Assoc Disord. 17:117-126.

DeLano, W.L. (2002) The PyMOL Molecular Graphics System. DeLano Scientific, San Carlos, CA, USA. http://www.pymol.org

Deutscher MP. (1993) Ribonuclease multiplicity, diversity, and complexity. I Biol Chem. 268:13011-13014.

Dieckmann T, Butcher SE, Sassanfar M, Szostak JW, Feigon J. (1997) Mutant ATP-binding RNA aptamers reveal the structural basis for ligand binding. J Mol Biol. 273:467-478.

Dixon MC. (2008) Quartz crystal microbalance with dissipation monitoring: enabling real-time characterization of biological materials and their interactions. J Biomol Tech. 19:151-158. 
Douglas SM, Marblestone AH, Teerapittayanon S, Vazquez A, Church GM, Shih WM. (2009) Rapid prototyping of 3D DNA-origami shapes with caDNAno. Nucleic Acids Res. 37:5001-5006.

Downard AJ, Roddick AD, Bond AM. (1995) Covalent modification of carbon electrodes for voltammetric differentiation of dopamine and ascorbic acid. Analytica Chimica Acta 317:303-310.

Dunker MFM, Starkey EB, Jenkins GL. (1936) The preparation of some organic mercurials from diazonium borofluorides J. Am. Chem. Soc. 58:2308-2309.

Dupont DM, Andersen LM, Botkjaer KA, Andreasen PA. (2011) Nucleic acid aptamers against proteases. Curr Med Chem. 18:4139-4151.

DuVall SH, McCreery RL. (1999) Control of Catechol and Hydróquinone Electron-Transfer Kinetics on Native and Modified Glassy Carbon Electrodes. Anal. Chem. 71:45944602.

Ellington AD, Szostak, JW. (1990) In vitro selection of RNA molecules that bind specific ligands. Nature $346,818-822$.

Esposito CL, Catuogno S, de Franciscis V, Cerchia L. (2011) New insight into clinical development of nucleic acid aptamers. Discov Med. 11:487-496.

Faludi G, Mirnics K. (2011) Synaptic changes in the brain of subjects with schizophrenia. Int J Dev Neurosci. 29:305-309. 
Famulok M. (1994) Molecular Recognition of Amino Acids by RNA-Aptamers: An LCitrulline Binding RNA Motif and Its Evolution into an L-Arginine Binder. J. Am. Chem. Soc., 116:1698-1706.

Fischer NO, Tarasow TM. (2011) Identification and optimization of DNA aptamer binding regions using DNA microarrays. Methods Mol Biol. 723:57-66.

Fylstra D, Lasdon L, Watson J, Waren A. (1998) Design and Use of the Microsoft Excel Solver. Interfaces 28:29-55.

Gan N, Xiao Y, Zhu L, Wu Z, Liu J, Hu C, Song L. (2012) The role of microcystins in maintaining colonies of bloom-forming Microcystis spp. Environ Microbiol. 2012 $14: 730-742$.

Geiger A, Burgstaller P, von der Eltz H, Roeder A, Famulok M. (1996) RNA aptamers that bind L-arginine with sub-micromolar dissociation constants and high enantioselectivity. Nucleic Acids Res. Mar 15;24(6):1029-36.

Geiger R (1984) Kallikrein. In: Bergmer HU, Bergmer J, GraßI M (eds) Methods of enzymatic analysis, vol 5. Verlag Chemic GmbH, Weinheim, pp 129-143.

Giacomellia C, Cklessb K, Galatoa D, Mirandaa FS, Spinelli A. (2002) Electrochemistry of Caffeic Acid Aqueous Solutions with pH 2.0 to 8.5. J. Braz. Chem. Soc. 13:332-338.

Gohara DW, Di Cera E. (2011) Allostery in trypsin-like proteases suggests new therapeutic strategies. Trends Biotechnol. 29:577-585. 
Gold L, Ayers D, Bertino J, Bock C, Bock A, Brody EN, Carter J, Dalby AB, Eaton BE, Fitzwater T, Flather D, Forbes A, Foreman T, Fowler C, Gawande B, Goss M, Gunn M, Gupta S, Halladay D, Heil J, Heilig J, Hicke B, Husar G, Janjic N, Jarvis T, Jennings S, Katilius E, Keeney TR, Kim N, Koch TH, Kraemer S, Kroiss L, Le N, Levine D, Lindsey W, Lollo B, Mayfield W, Mehan M, Mehler R, Nelson SK, Nelson M, Nieuwlandt D, Nikrad M, Ochsner U, Ostroff RM, Otis M, Parker T, Pietrasiewicz S, Resnicow DI, Rohloff J, Sanders G, Sattin S, Schneider D, Singer B, Stanton M, Sterkel A, Stewart A, Stratford S, Vaught JD, Vrkljan M, Walker JJ, Watrobka M, Waugh S, Weiss A, Wilcox SK, Wolfson A, Wolk SK, Zhang C, Zichi D. (2010) Aptamer-based multiplexed proteomic technology for biomarker discovery. PLoS One. 5:e15004.

Gold L, Polisky B, Uhlenbeck O, Yarus M. (1995) Diverșity of oligonucleotide functions. Annu Rev Biochem. 64:763-797.

Gonon F, Buda M, Cespuglio R, Jouvet M, Pujol JF. (1980) In vivo electrochemical detection of catechols in the neostriatum of anaesthetized rats: dopamine or DOPAC? Nature. 286:902-904.

Graham JC, Zarb| H. (2012) Use of cell-SELEX to generate DNA aptamers as molecular probes of HPV-associated cervical cancer cells. PLoS One. 7:e36103

Gruber AR, Lorenz R, Bernhart SH, Neuböck R, Hofacker IL. (2008) The Vienna RNA Websuite. Nucleic Acids Res. 36:W70-74. 
Gruber AR, Lorenz R, Bernhart SH, Neuböck R, Hofacker IL. (2008) The Vienna RNA Websuite. Nucleic Acids Res. 36:W70-74.

Gu KD, Famulok M. (2004) In vitro selection of specific aptamers against microcystin-LR. Zhonghua Yu Fang Yi Xue Za Zhi. 38:369-373.

Ha TH, Kim S, Lim G, Kim K. (2004) Influence of liquid medium and surface morphology on the response of QCM during immobilization and hybridization of short oligonucleotides. Biosens Bioelectron. 20:378-389.

Hannoush RN, Damha MJ. (2001) Remarkable stability of hairpins containing 2',5'-linked RNA loops. J Am Chem Soc. 123:12368-12374.

Harper JC, Polsky R, Wheeler DR, Brozik SM. (2008) Maleimide-activated aryl diazonium salts for electrode surface functionalization with biological and redox-active molecules. Langmuir 24:2206-2211.

Hélène C, Montenay-Garestier T, Dimicoli JL. (1971) Interactions of tyrosine and tyramine with nucleic acids and their components. Fluorescence, nuclear magnetic resonance and circular dichroism studies. Biochim Biophys Acta. 254:349-65.

Hermann T, Patel DJ. (2000) Adaptive recognition by nucleic acid aptamers. Science. 287:820-825.

Herráez A. (2006) Biomolecules in the computer: Jmol to the rescue. Biochem Mol Biol Educ. 34:255-261 
Herranz S, Marazuela MD, Moreno-Bondi MC. (2012) Automated portable array biosensor for multisample microcystin analysis in freshwater samples. Biosens Bioelectron. 33:50-55.

Hill AV. (1910) The possible effects of the aggregation of the molecules of huemoglobin on its dissociation curves. J. Physiol. (Lond.) 40(Suppl):iv-vii.

Holahan MR, Madularu D, McConnell EM, Walsh R, DeRosa MC. (2011) Intra-accumbens injection of a dopamine aptamer abates MK-801-induced cognitive dysfunction in a model of schizophrenia. PLoS One. 6:e22239.

Hoss W, Smiley C. (1977) Binding and immobilization of catecholamines by liposomes. J Neurosci Res. 3:249-256.

Htun H, Dahlberg JE. (1989) Topology and formation of triple-stranded H-DNA. Science. 243:1571-1576.

Huizenga DE, Szostak JW. (1995) A DNA aptamer that binds adenosine and ATP. Biochemistry. 34:656-665.

Humpage AR, Froscio SM, Lau HM, Murphy D, Blackbeard J. (2012) Evaluation of the Abraxis Strip Test for Microcystins ${ }^{\mathrm{TM}}$ for use with wastewater effluent and reservoir water. Water Res. 46:1556-1565.

Iliuk AB, Hu L, Tao WA. (2011) Aptamer in bioanalytical applications. Anal Chem. 83:4440-4452. 
Jameson DM, Ross JA. (2010) Fluorescence polarization/anisotropy in diagnostics and imaging. Chem Rev. 110:2685-2708.

Jayasena SD. (1999) Aptamers: an emerging class of molecules that rival antibodies in diagnostics. Clin Chem. 45:1628-1650.

Jeffries AC, Symons RH. (1989) A catalytic 13-mer ribozyme. Nucleic Acids Res. 17:13711377.

Jing M, Bowser MT. (2011) Methods for measuring aptamer-protein equilibria: a review. Anal Chim Acta. 686:9-18

Johnson M, Zaretskaya I, Raytselis Y, Merezhuk Y, McGinnis S, Madden TL. (2008) NCBI BLAST: a better web interface. Nucleic Acids Res. 36(Web Server issue):W5-9.

Joyce GF. (2009) Evolution in an RNA world. Cold Spring Harb Symp Quant Biol. 74:1723.

Kang H, Liu H, Phillips JA, Cao Z, Kim Y, Chen Y, Yang Z, Li J, Tan W. (2009) Single-DNA molecule nanomotor regulated by photons. Nano Lett. 9:2690-2696.

Kazitsyna LA, Kikot' BS, Reutov OA. (1964) Infrared absorption spectra of solutions of diazonium salts in the region of $2200-2300 \mathrm{~cm}^{-1}$. Russ Chem B. $13: 894-897$

Kelly RS, Weiss DJ, Chong SH, Kuwana T. (1999) Charge-selective electrochemistry at high-surface-area carbon fibers. Analytical Chemistry 71:413-418. 
Kemmer G, Keller S. (2010) Nonlinear least-squares data fitting in Excel spreadsheets. Nat. Protoc. 5:267-281.

Kim HR, Kim TH, Hong SH, Kim HG. (2012) Direct detection of tetrahydrobiopterin (BH4) and dopamine in rat brain using liquid chromatography coupled electrospray tandem mass spectrometry. Biochem Biophys Res Commun. 419:632-637.

Klein TA, Neumann J, Reuter M, Hennig J, von Cramon DY, Ullsperger M. (2007) Genetically determined differences in learning from errors. Science 318:1642-1645.

Krishnamurthy T, Carmichael WW, Sarver EW. (1986) Toxic peptides from freshwater cyanobacteria (blue-green algae). I. Isolation, purification and characterization of peptides from Microcystis aeruginosa and Anabaena flos-aquae. Toxicon. 24:865873.

Krylova SM, Koshkin V, Bagg E, Schofield CJ, Krylov SN. (2012) Mechanistic studies on the application of DNA aptamers as inhibitors of 2-oxoglutarate-dependent oxygenases. J Med Chem. 55:3546-3552.

Kukor Z, Mayerle J, Krüger B, Tóth M, Steed PM, Halangk W, Lerch MM, Sahin-Tóth M. (2002) Presence of cathepsin B in the human pancreatic secretory pathway and its role in trypsinogen activation during hereditary pancreatitis. J Biol Chem. 277:21389-21396. 
Kurosawa S, Park JW, Aizawa H, Wakida S, Tao H, Ishihara K. (2006) Quartz crystal microbalance immunosensors for environmental monitoring. Biosens Bioelectron. 22:473-481.

Kuzuya A, Komiyama M. (2010) DNA origami: fold, stick, and beyond. Nanoscale. 2:310322.

Lauhon CT, Szostak JW. (1995) RNA aptamers that bind flavin and nicotinamide redox cofactors. J Am Chem Soc. 117:1246-1257.

Lavery R, Zakrzewska K, Sun IS, Harvey SC. (1992) A comprehensive classification of nucleic acid structural families based on strand direction and base pairing. Nucleic Acids Res. 20:5011-5016.

Le Bousse-Kerdilès MC, Martyré MC, Samson M. (2008) Cellular and molecular mechanisms underlying bone marrow and liver fibrosis: a review. Eur Cytokine Netw. 19:69-80.

Lee JA, DeRosa MC. (2010) A pH-driven DNA switch based on the A+ $x \mathrm{G}$ mispair. Chem Commun (Camb). 46:418-420.

Lees AJ, Hardy J, Revesz T. (2009) Parkinson's disease. Lancet 373: 2055-2066.

Lent CM, Mueller RL, Haycock DA. (1983) Chromatographic and histochemical identification of dopamine within an identified neuron in the leech nervous system. I Neurochem. 41(2):481-490. 
Leontis NB, Stombaugh J, Westhof E. (2002) The non-Watson-Crick base pairs and their associated isostericity matrices. Nucleic Acids Res. 30:3497-3531.

Lichtarge O, Wilkins A. (2010) Evolution: a guide to perturb protein function and networks. Curr Opin Struct Biol. 20:351-359.

Lin PH, Yen SL, Lin MS, Chang Y, Louis SR, Higuchi A, Chen WY. (2008) Microcalorimetrics studies of the thermodynamics and binding mechanism between L-tyrosinamide and aptamer. J Phys Chem B. 112:6665-6673.

Lin YW, Liu CW, Chang HT. (2011) Fluorescence detection of mercury(II) and lead(II) ions using aptamer/reporter conjugates. Talanta. 84:324-329.

Linak MC, Tourdot R, Dorfman KD. (2011) Moving beyond Watson-Crick models of coarse grained DNA dynamics. J Chem Phys. 135:205102.

Liu A, Wang E. (1994) Determination of catechol derivatives on pretreatment and copolymer coated glassy carbon electrode. Talanta. 41:147-154

Liu H, Liu D (2009) DNA nanomachines and their functional evolution. Chem Comm. 19:2625-2636.

Liu T, Tang J, Jiang L. (2002) Sensitivity enhancemerit of DNA sensors by nanogold surface modification. Biochem Biophys Res Commun. 295:14-16.

Lorsch JR, Szostak JW. (1994) In vitro selection of RNA aptamers specific for cyanocobalamin. Biochemistry. 33:973-982. 
Lu X-J, Olson WK (2008) 3DNA: a versatile, integrated software system for the analysis, rebuilding and visualization of three-dimensional nucleic-acid structures. Nat. Protoc. 3:1213-1227.

Lu X-J, Shakked Z, Olson WK (2000) A-form conformational motifs in ligand-bound DNA structures. J. Mol. Biol. 300:819-840.

Luo X, Mckeague M, Pitre S, Dumontier M, Green J, Golshani A, Derosa MC, Dehne F. (2010) Computational approaches toward the design of pools for the in vitro selection of complex aptamers. RNA. 16:2252-2262.

Malik FI, Hartman JJ, Elias KA, Morgan BP, Rodriguez H, Brejc K, Anderson RL, Sueoka SH, Lee KH, Finer JT, Sakowicz R, Baliga R, Cox DR, Garard M, Godinez G, Kawas R, Kraynack E, Lenzi D, Lu PP, Muci A, Niu C, Qian X, Pierce DW, Pokrovskii M, Suehiro I, Sylvester S, Tochimoto T, Valdez C, Wang W, Katori T, Kass DA, Shen YT, Vatner SF, Morgans DJ. (2011) Cardiac myosin activation: a potential therapeutic approach for systolic heart failure. Science. 331:1439-1443.

Mann TL, Krull UJ. (2003) Fluorescence polarization spectroscopy in protein analysis. Analyst. 128:313-317.

Mannironi C, Di Nardo A, Fruscoloni P, Tocchini-Valentini GP. (1997) In vitro selection of dopamine RNA ligands. Biochemistry. 36:9726-9734. 
Mas M, Gonzalez-Mora JL, Hernandez L. (1996) In vivo monitoring of brain neurotransmitter release for the assessment of neuroendocrine interactions. Cell Mol Neurobiol. 16:383-396.

Mascini M, Palchetti I, Tombelli S. (2012) Nucleic acid and peptide aptamers: fundamentals and bioanalytical aspects. Angew Chem Int Ed Engl. 51:1316-1332.

Matschinsky FM, Zelent B, Doliba N, Li C, Vanderkooi JM, Naji A, Sarabu R, Grimsby J. (2011) Glucokinase activators for diabetes therapy: May 2010 status report. Diabetes Care. 34:S236-S243.

Mayer G, Rohrbach F, Pötzsch B, Müller J. (2011) Aptamer-based modulation of blood coagulation. Hamostaseologie. 31:258-263.

Mccreery RL.(2008) Advanced carbon electrode materials for molecular electrochemistry. Chem Rev 108:2646-2687.

McKeague M, DeRosaMC. (2012) Challenges and opportunities for small molecule aptamer development. JNA (in press).Mok.W, Li Y. (2008) Recent Progress in Nucleic Acid Aptamer-Based Biosensors and Bioassays. Sensors 8:7050-7084.

Motulsky HJ, Christopoulos A. (2004) Fitting ṃodels to biological data using linear and nonlinear regression, Oxford University Press, NY, USA.

Msagati TA, Siame BA, Shushu DD. (2006) Évaluation of methods for the isolation, $\therefore$ detection and quantification of cyanobacterial hepatotoxins. Aquat Toxicol. 78:382397. 
Myers RD, Adell A, Lankford MF. (1988) Simultaneous comparison of cerebral dialysis and push-pull perfusion in the brain of rats: a critical review. Neurosci Biobehav Rev. 22:371-387.

Nakamuraa C, Kobayashiab T, Miyakea M, Shiraib M, Miyakeaa J. (2001) Usage of a DNA Aptamer as a Ligand Targeting Microcystin. Mol. Cryst. Liquid Cryst. 371:369-374.

Nenov IP, Fylstra DH. (2003) Interval Methods for Accelerated Global Search in the Microsoft Excel Solver. Reliab. Comput. 9:143-159.

Nicholson RS, Irving. Shain (1964) Theory of Stationary Electrode Polarography. Single Scan and Cyclic Methods Applied to Revèrsible, Irreversible, and Kinetic Systems. Anal. Chem. 36:706-723.

Ogston AG. (1948) Interpretation of experiments on metabolic processes, using isotopic tracer elements. Nature. 162:963.

Ohnsorg PM, Mary JL, Rohrer L, Pech M, Fingerle J, von Eckardstein A. (2011) Trimerized apolipoprotein A-I (TripA) forms lipoproteins, activates lecithin: cholesterol acyltransferase, elicits lipid efflux, and is transported through aortic endothelial cells. Biochim Biophys Acta. 1811:1115-1123.

Page MJ, Di Cera E. (2008) Serine peptidases: classification, structure and function. Cell Mol Life Sci. 65:1220-1236. 
Pagratis NC, Bell C, Chang YF, Jennings S, Fitzwater T, Jellinek D, Dang C. (1997) Potent 2'-amino-, and 2'-fluoro-2'-deoxyribonucleotide RNA inhibitors of keratinocyte growth factor. Nat Biotechnol. 15:68-73.

Parisien M, Major F. (2008) The MC-Fold and MC-Sym pipeline infers RNA structure from sequence data. Nature. 452:51-55.

Parkinson GN. Fundamentals of quadruplex structures. In Quadruplex nucleic acids. Edited by Neidle S, Balasubramanian S. Cambridge, UK: RSC Publishing; 2006:1-30.

Paul N, Springsteen G, Joyce GF. (2006) Conversion of a ribozyme to a deoxyribozyme through in vitro evolution. Chem Biol. 13:329-338.

Pearson L, Mihali T, Moffitt M, Kellmann R, Neilan B. (2010) On the chemistry, toxicology and genetics of the cyanobacterial toxins, microcystin, riodularin, saxitoxin and cylindrospermopsin. Mar Drugs. 8:1650-1680Caré BR,

Phizicky EM, Fields S. (1995) Protein-protein interactions: methods for detection and analysis. Microbiol Rev. 59:94-123.

Ping J, Wu J, Wang Y, Ying Y. (2012) Simultaneous determination of ascorbic acid, dopamine and uric acid using high-performance screen-printed graphene electrode. Biosens Bioelectron. 34:70-76.

Priyakumar UD, Hyeon C, Thirumalai D, Mackerell AD Jr. (2009) Urea destabilizes RNA by forming stacking interactions and multiple hydrogen bonds with nucleic acid bases. J Am Chem Soc. 131:17759-17761. 
Rahimi F, Bitan G. (2010) Selection of aptamers for amyloid beta-protein, the causative agent of Alzheimer's disease. J Vis Exp. 13;(39). pii: 1955.

Rahman MA, Shiddiky MJ, Park JS, Shim YB. (2007) An Impedimetric immunosensor for the label-free detection of bisphenol A. Biosens Bioelectron. 22:2464-2470.

Ramaley L, Krause Jr. MS. (1969) Theory of square wave voltammetry. Anal. Chem. 41:1362-1365.

Sakata T, Hiroaki H, Oda Y, Tanaka T, Ikehara M, Uesugi S. (1990) Studies on the structure and stabilizing factor of the CUUCGG hairpin RNA using chemically synthesized oligonucleotides. Nucleic Acids Res. 18:3831-3839.

Salazar M, Fedoroff OY, Miller JM, Ribeiro NS, Reid BR. (1993). The DNA strand in DNA.RNA hybrid duplexes is neither B-form nor A-form in solution. Biochemistry. $32: 4207-4215$.

Salim NN, Feig AL. (2009) Isothermal titration calorimetry of RNA. Methods. 47:198-205.

Sambrook, J., Fritsch, E.F., Manitis, T., Irwin, N, Polyacrylamide Gel Electrophoresis From: Molecular Cloning 2nd Edition 1989, USA.

Sauerbrey G. (1959) Verwendung von Schwingquarzen zur Wägung dünner Schichten und zur Microwägang. Z. Phys:' 155:206-212.

Schwartz DK. (2001) Mechanisms and kinetics of self-assembled monolayer formation. Annu Rev Phys Chem. 52:107-137. 
Shearer G Jr. (1994) A syringe-based gradient former for linear and exponential gradients. Anal Biochem. 221:397-400.

Sheng ZH, Zheng XQ, Xu JY, Bao WJ, Wang FB, Xia XH. (2012) Electrochemical sensor based on nitrogen doped graphene: simultaneous determination of ascorbic acid, dopamine and uric acid. Biosens Bioelectron. 34:125-131.

Shulman JM, De Jager PL, Feany MB. (2011) Parkinson's disease: genetics and pathogenesis. Annu Rev Pathol. 6:193-222.

Siddique MM, Tan EK. (2010) Neurochemistry changes associated with mutations in familial Parkinson's disease. Curr Med Chem. 17:4378-4391.

Silverman SK. (2009) Deoxyribozymes: selection design and serendipity in the development of DNA catalysts. Acc Chem Res. 42:1521-1531.

Silverman SK. Artificial functional nucleic acids: aptamers, ribozymes and deoxyribozymes identified by in vitro selection, in: Y. Li, Y. Lu (Eds.), Functional Nucleic Acids for Analytical Applications, Springer. Science + Business Media, LLC, New York, 2009, pp. 47-108.

Soontornworajit B, Wang Y. (2011) Nucleic acid aptamers for clinical diagnosis: cell detection and molecular imaging. Anal Bioanal Chem. 399:1591-1599.

Soula HA. (2011) Impact of receptor clustering on ligand binding. BMC Syst Biol. 5:48. 
Stojanovic MN, de Prada P, Landry DW. (2001) Aptamer-based folding fluorescent sensor for cocaine. J Am Chem Soc. 123:4928-4931.

Stoltenburg R, Reinemann C, Strehlitz B. (2005) FluMag-SELEX as an advantageous method for DNA aptamer selection. Anal Bioanal Chem. 383:83-91.

Stoltenburg R, Reinemann C, Strehlitz B. (2007) SELEX--a (r)evolutionary method to generate high-affinity nucleic acid ligands. Biomol Eng. 24:381-403.

Sultan Y, DeRosa MC. (2011) Target binding influences permeability in aptamerpolyelectrolyte microcapsules. Small. 7:1219-1226.

Sun D, Hurley LH. (2009) The importance of negative superhelicity in inducing the formation of G-quadruplex and i-motif structures in the c-Myc promoter: implications for drug targeting and control of gene expression. J Med Chem. $52: 2863-2874$.

Sussman D, Nix JC, Wilson C. (2000) The structural basis for molecular recognition by the vitamin B 12 RNA aptamer. Nat Struct Biol. 7:53-57.

Svircev Z, Krstic S, Miladinov-Mikov M, Baltic V, Vidovic M. (2009) Freshwater cyanobacterial blooms and primary liver cancer epidemiological studies in Serbia. J Environ Sci Health C Environ Carcinog Ecotoxicol Rev. 27:36-55.

Swamy BE, Venton BJ. (2007) Carbon nanotube-modified microelectrodes for simultaneous detection of dopamine and serotonin in vivo. Analyst. 132:876-884. 
Tahiri-Alaoui A, Frigotto L, Manville N, Ibrahim J, Romby P, James W. (2002) High affinity nucleic acid aptamers for streptavidin incorporated into bi-specific capture ligands. Nucleic Acids Res. 30 e45.

Tan W, Wang H, Chen $Y$, Zhang $X$, Zhu $H$, Yang C, Yang R, Liu C. (2011) Molecular aptamers for drug delivery. Trends Biotechnol. 29:634-640.

Tian X, Cheng C, Yuan H, Du J, Xiao D, Xie S, Choi MM. (2012) Simultaneous determination of I-ascorbic acid, dopamine and uric acid with gold nanoparticles- $\beta$ cyclodextrin-graphene-modified electrode by square wave voltammetry. Talanta. 93:79-85.

Travascio P, Bennet AJ, Wang DY, Sen D. (1999) A ribozyme and a catalytic DNA with peroxidase activity: active sites versus cofactor-binding sites. Chem Biol. 6:779-787.

Tsai HY, Lin ZH, Chang HT. (2012) Tellurium-nanowire-coated glassy carbon electrodes for selective and sensitive detection of dopamine. Biosens Bioelectron. 35:479-483.

Wachter A. (2010) Riboswitch-mediated control of gene expression in eukaryotes. RNA Biol. 7:67-76.

Walsh R, DeRosa MC. (2009) Retention of function in the DNA homolog of the RNA dopamine aptamer. Biochem Biophys Res Commun. 388:732-735.

Walsh R, Martin E, Darvesh S. (2011) Limitations of conventional inhibitor classifications. Integr Biol (Camb). 3:1197-1201. 
Walsh R. (2012) Alternative Perspectives of Enzyme Kinetic Modeling, Medicinal Chemistry and Drug Design, Deniz Ekinci (Ed.), ISBN: 978-953-51-0513-8, InTech, Available from: http://www.intechopen.com/books/medicinal-chemistry-and-drugdesign/alternative-perspectives-of-enzyme-kinetic-modeling

Walther WG, Edmunds LN. (1970) Periodic increase in deoxyribonuclease activity during the cell cycle in synchronized euglena. J Cell Biol. 46:613-617.

Wang ZG, Wilner OI, Willner I. (2009) Self-assembly of aptamer-circular DNA nanostructures for controlled biocatalysis. Nano Lett. 9:4098-4102.

Watkins PB, Zimmerman HJ, Knapp MJ, Gracon SI, Lewis KW. (1994) Hepatotoxic effects of tacrine administration in patients with Alzheimer's disease. JAMA. 271:992-998.

Wilchek M, Knudsen KL, Miron T. (1994) Improved method for preparing Nhydroxysuccinimide ester-containing polymers for affinity chromatography. Bioconjug Chem. 5:491-492.

Wilson K, Walker J. (2005) Principles and Techniques of Biochemistry and Molecular Biology. Cambridge University Press.

Wolfenden R. (2011) Benchmark reaction rates, the stability of biological molecules in water, and the evolution of catalytic power in enzymes. Annu Rev Biochem. 80:645667.

Wu Y, Sefah K, Liu H, Wang R, Tan W. (2010) DNA aptamer-micelle as an efficient detection/delivery vehicle toward cancer cells. Proc Natl Acad Sci U S A. 107:5-10. 
Yang W, Baker SE, Butler JE, Lee C, Russell Jr. JN, Shang L, Sun B, Hamers RJ. (2005) Electrically addressable biomolecular functionalization of conductive nanocrystalline diamond thin films. Chem. Mater. 17:938-940.

Yoo SM, Choi JH, Lee SY, Yoo NC. (2009) Applications of DNA microarray in disease diagnostics. J Microbiol Biotechnol. 19:635-646.

Zachek MK, Park J, Takmakov P, Wightman RM, McCarty GS. (2010). Microfabricated FSCV-compatible microelectrode array for real-time monitoring of heterogeneous dopamine release. Analyst. 135:1556-1563

Zamyadi A, MacLeod SL, Fan Y, McQuaid N, Dorner S, Sauvé S, Prévost M. (2012) Toxic cyanobacterial breakthrough and accumulation in a drinking water plant: a monitoring and treatment challenge. Water Res. 2012 46:1511-1523.

Zhang J, Lau MW, Ferré-D'Amaré AR. (2010) Ribozymes and riboswitches: modulation of RNA function by small molecules. Biochemistry. 49:9123-9131.

Zhang L, Lin X. (2001) Covalent modification of glassy carbon electrodes with glycine for voltammetric separation of dopamine and ascorbic acid. Fresenius J Anal Chem. 370:956-962.

Zhang L, Sun YG. (2001) Covalent modification of glassy carbon electrodes with betaalanine for voltammetric separation of dopamine and ascorbic acid. Anal Sci. 17:939-943. 
Zheng G, Lu XJ, Olson WK. (2009) Web 3DNA--a web server for the analysis, reconstruction, and visualization of three-dimensional nucleic-acid structures. Nucleic Acids Res. 37(Web Server issue):W240-246.

Zheng Y, Wang Y, Yang X. (2011) Aptamer-based colorimetric biosensing of dopamine using unmodified gold nanoparticles. Sensors and Actuators, B: Chemical 156:95-99.

Zhou L, Yu H, Chen K. (2002) Relationship between microcystin in drinking water and colorectal cancer.Biomed Environ Sci. 15:166-171. 


\section{Appendix}

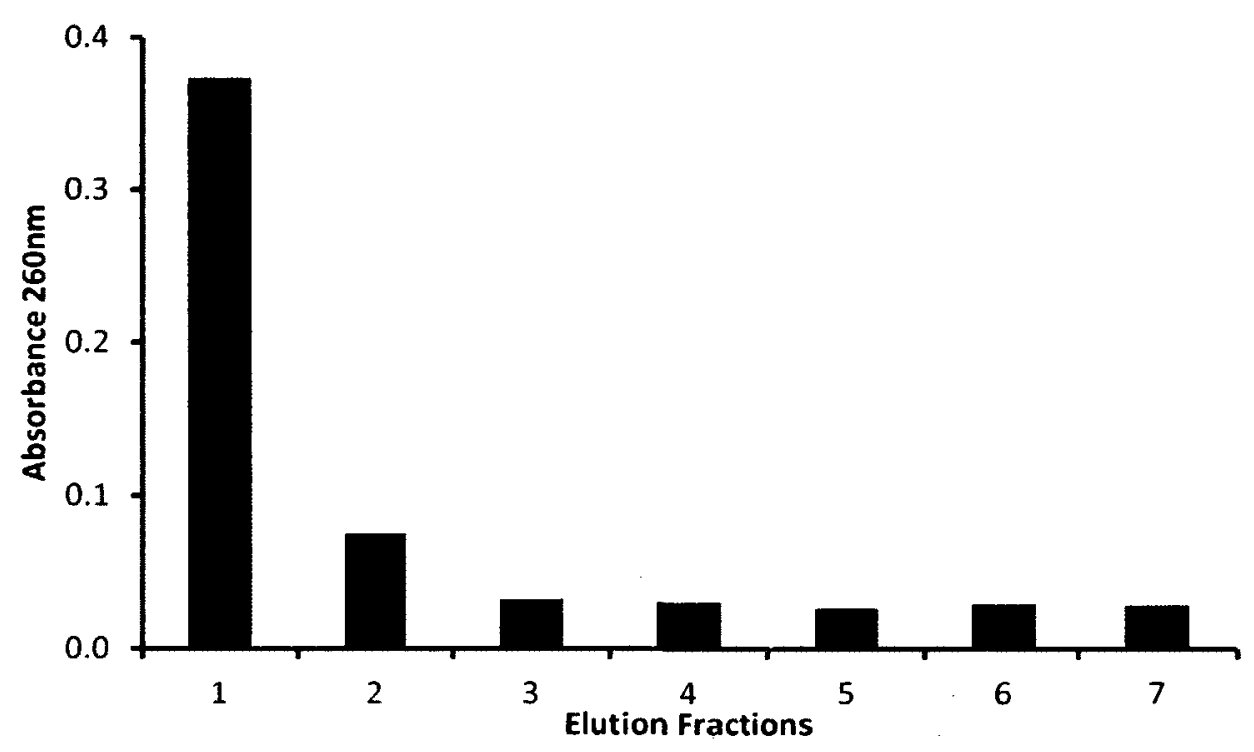

Appx Figure 1. Elution of the DNA homolog of the RNA B12 aptamer from the B12 column. The DNA was eluted in $1.5 \mathrm{~mL}$ fractions from the column using $3.5 \mathrm{M}$ urea, $90^{\circ} \mathrm{C}$. 


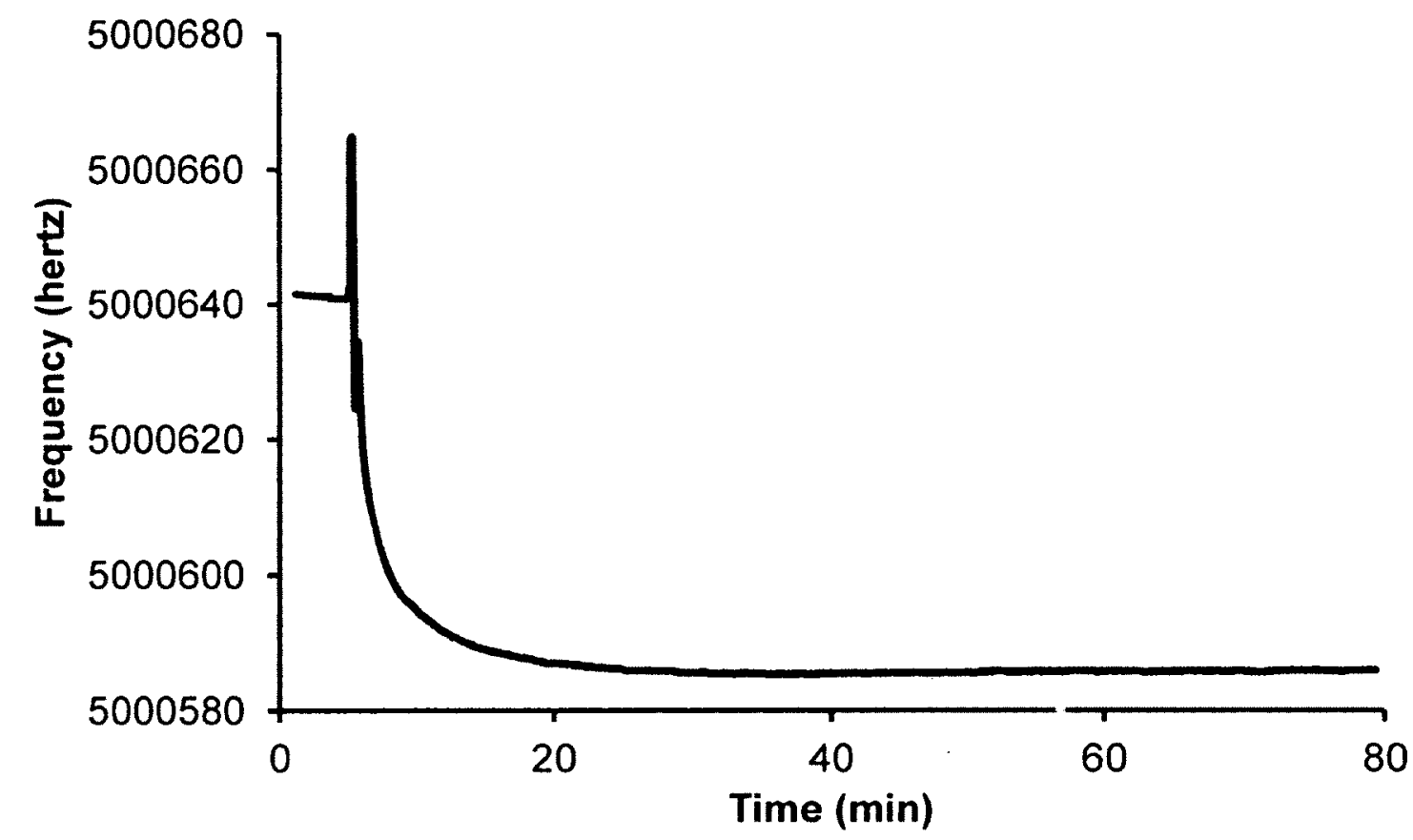

Appx Figure 2. Deposition of 6-Mercaptohexanol on the gold electrode pad of the QCM crystal. Decrease in frequency at which the QCM crystal vibrated was used to monitor the deposition of the organic monolayer from a $0.1 \mathrm{M} \mathrm{6-mercaptohexanol} \mathrm{solution} \mathrm{in}$ buffer. 


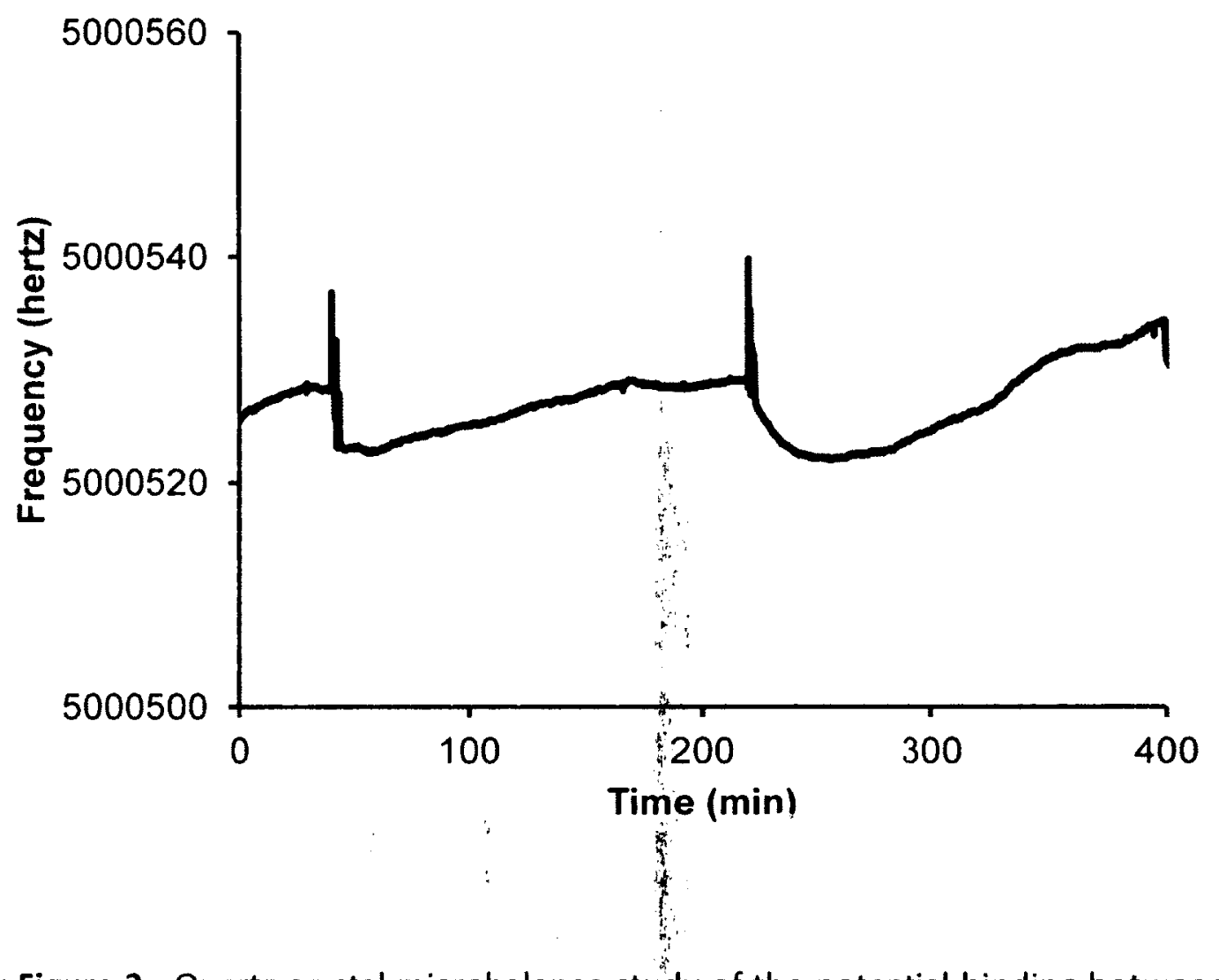

Appx Figure 3. Quartz crystal microbalance study of the potential binding between the aptamer and dopamine on the 6-Mercaptohexanol backfilled QCM crystal. The frequency of the aptamer functionalized crystal was monitored as increasing i: concentrations of dopamine were introduced to the flow cell $(0,0.87$ and $870 \mu \mathrm{M})$. 

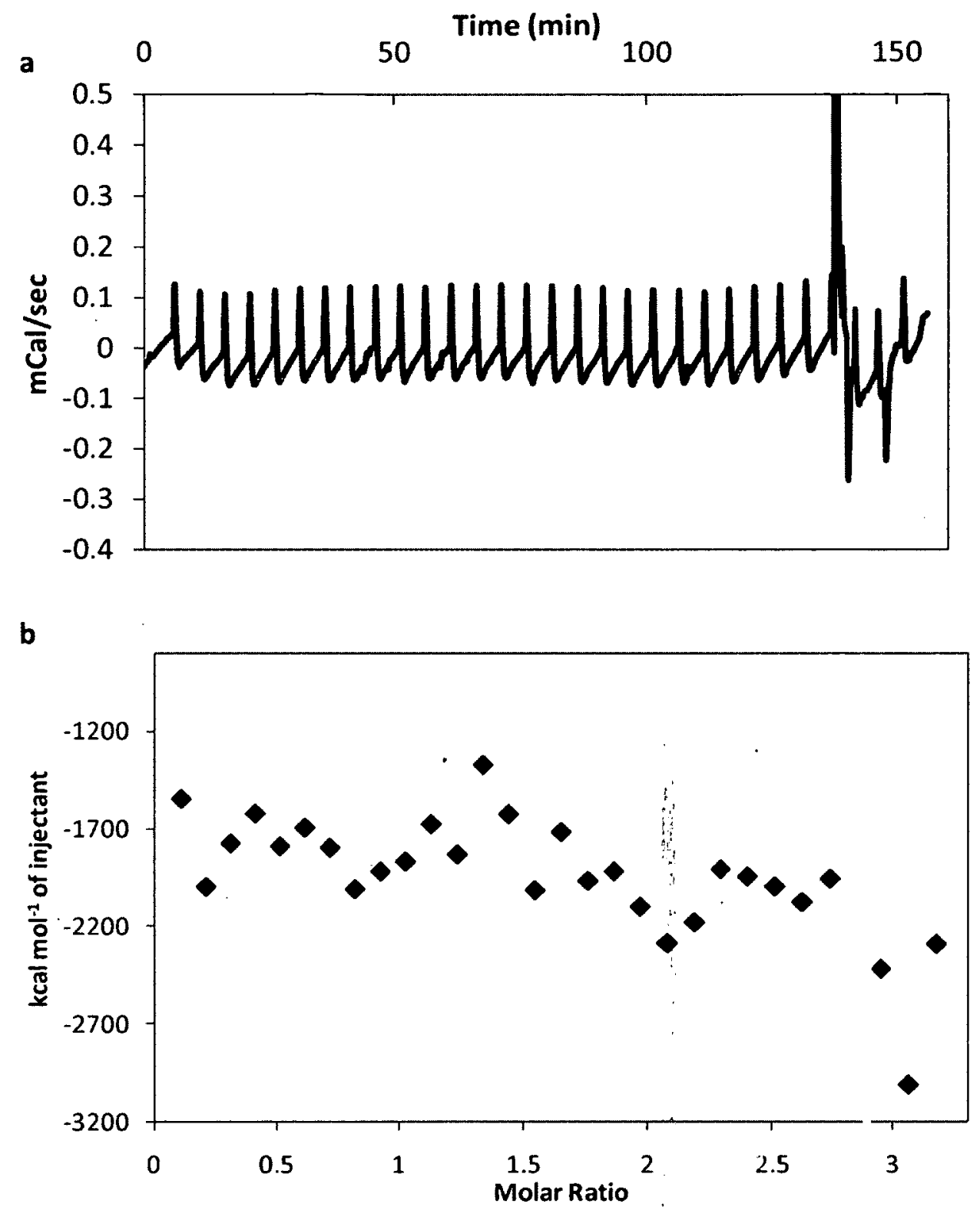

Appx Figure 4. Repeat of the isothermal titration microcalorimetric (a) raw data for the analysis of potential dopamine and aptamer interactions using thirty $6 \mu$ injections of $0.47 \mathrm{mM}$ dopamine into $1.4 \mathrm{mLs}$ of $20 \mu \mathrm{M}$ dopamine aptamer and (b) the associated integrated heat plot. 

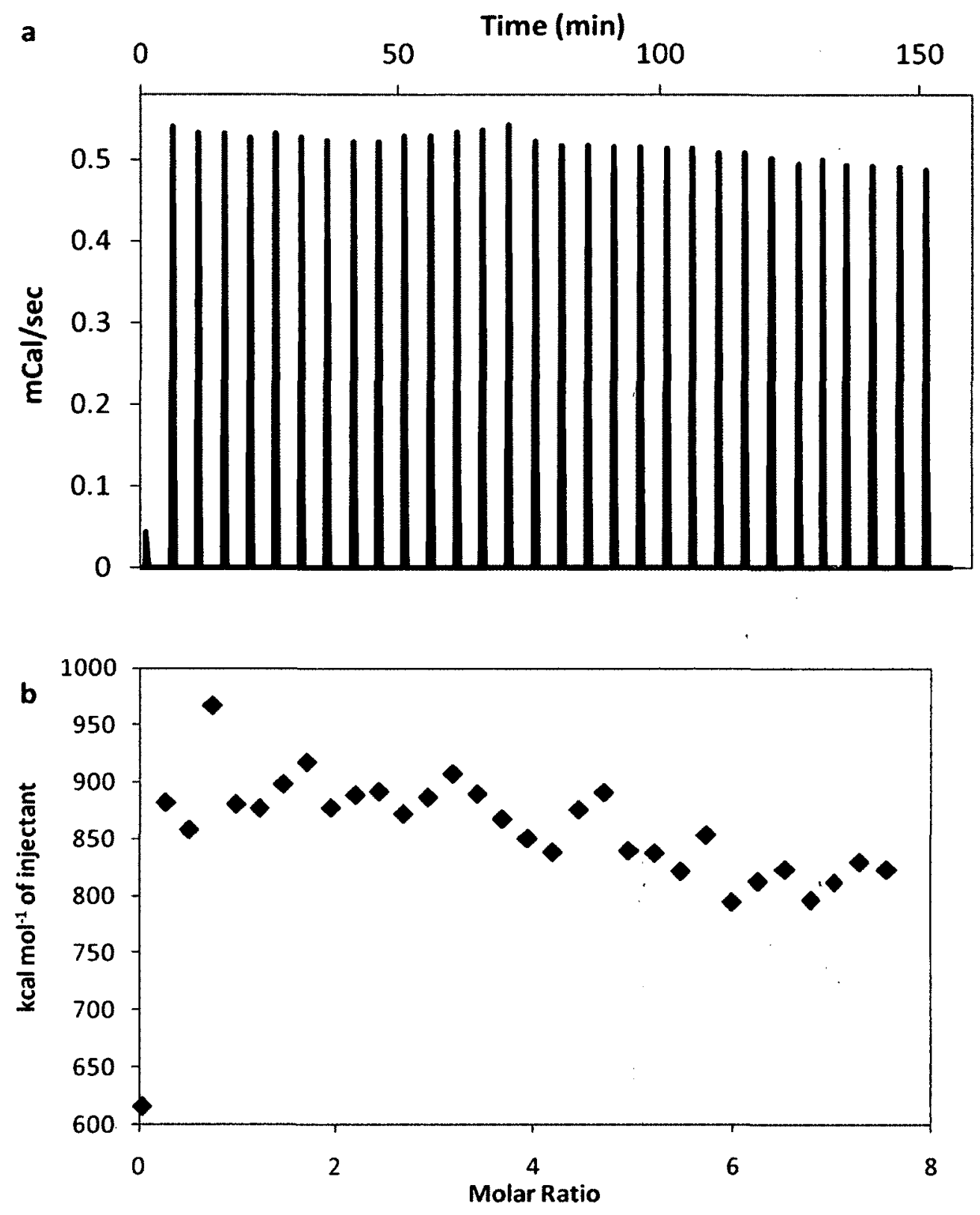

Appx Figure 5. Repeat of the isothermal titration microcalorimetric with ascorbic acid omitted from the buffer (a) raw data for the analysis of potential dopamine and aptamer interactions using thirty $6 \mu$ injections of $0.47 \mathrm{mM}$ dopamine into $1.4 \mathrm{mLs}$ of 20 $\mu \mathrm{M}$ dopamine aptamer and (b) the associated integrated heat plot. 


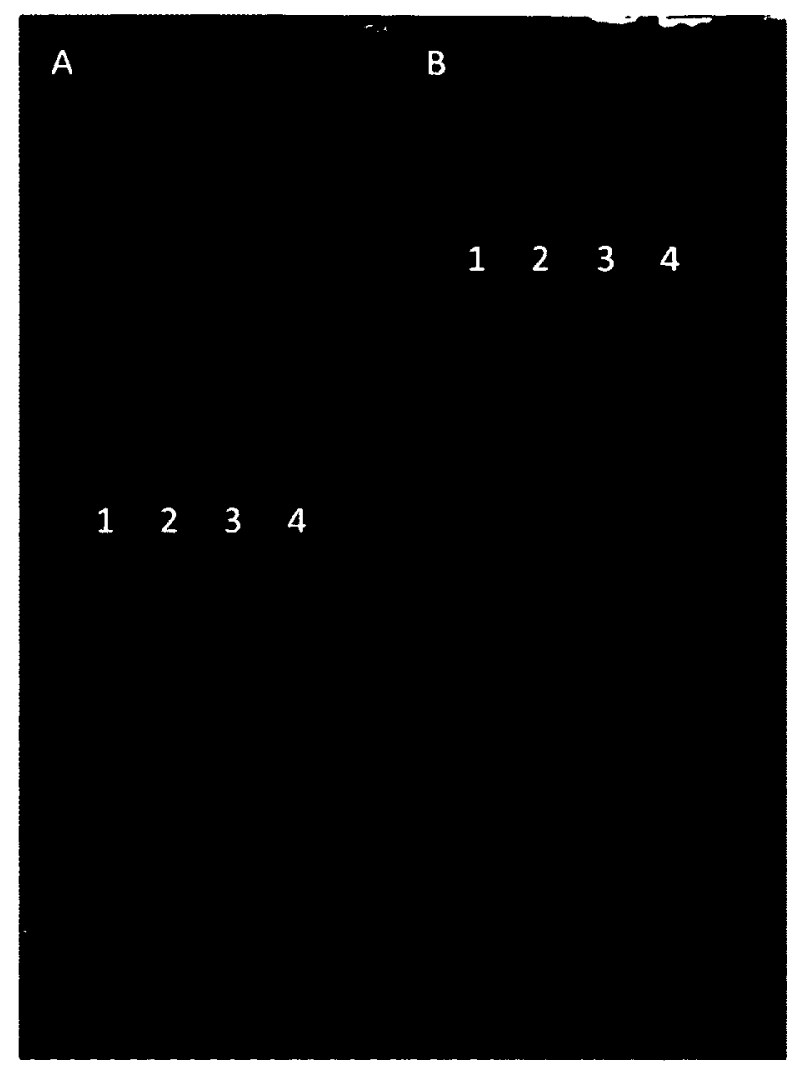

Appx Figure 6. Fluorescein labelled DNA homologue of the RNA Dopamine aptamer (4 $\mu \mathrm{M})$ run on A) a continuous gradient gel from $12-19 \%$ acrylamide gel and B) a $19 \%$ gel at a constant $35 \mathrm{mAmps}$ for 21 hours. Lane 1 and 2 contain just the aptamer while lane 3 and 4 were run in the presence of $10 \mathrm{mM}$ dopamine to ensure the presence of the dopamine bound conformation. The relative fluorescence of the fluorescein labelled aptamer was examined with an Alphalmáger EC System equip with a 537/35 nm filter. 


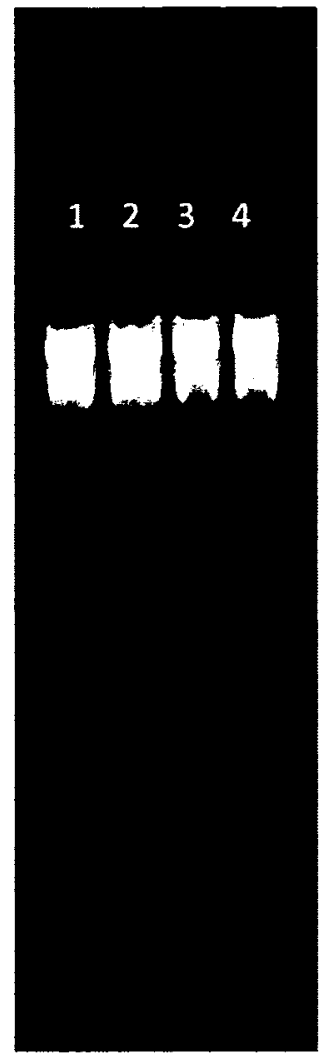

Appx Figure 7. Fluorescein labelled DNA homologue of the RNA Dopamine aptamer (4 $\mu \mathrm{M})$ run on an $8 \%$ acrylamide gel at a constant $45 \mathrm{mAmps}$ for 2 hours. Lane 1 and 2 contain just the aptamer while lane 3 and 4 were run in the presence of $100 \mathrm{mM}$ dopamine to ensure the presence of the dopamine bound conformation. The relative fluorescence of the fluorescein labelled aptamer was examined with an Alphalmager EC System equip with a $537 / 35 \mathrm{~nm}$ filter. 


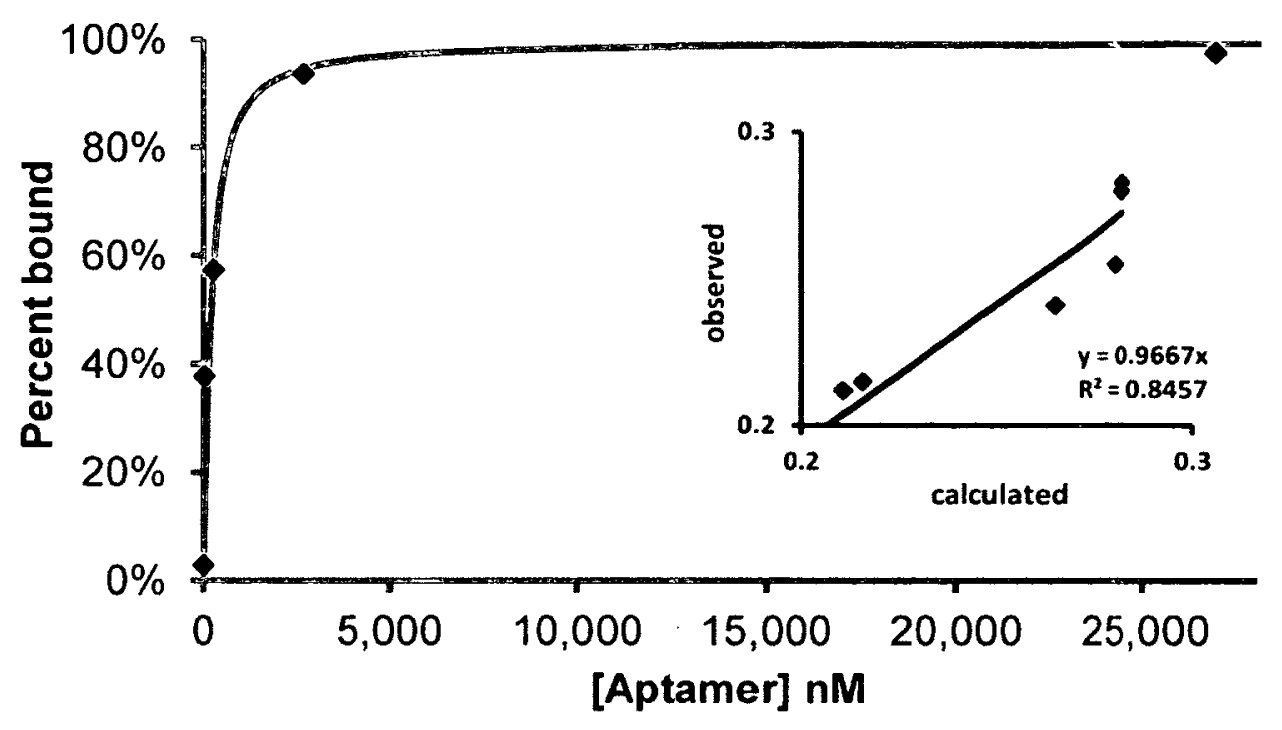

Appx Figure 8. Fluorescent anisotropy binding curve for the DNA form of the RNA dopamine aptamer and norepinephrine. Anisotropy values were collected using 0.8 aM norepinephrine which was exposed to increasing concentrations of the aptamer $(0.27$, $2.7,27,270,2700,27000 \mathrm{nM})$. The inset shows the agreement between the observed and the calculated anisotropy values. 


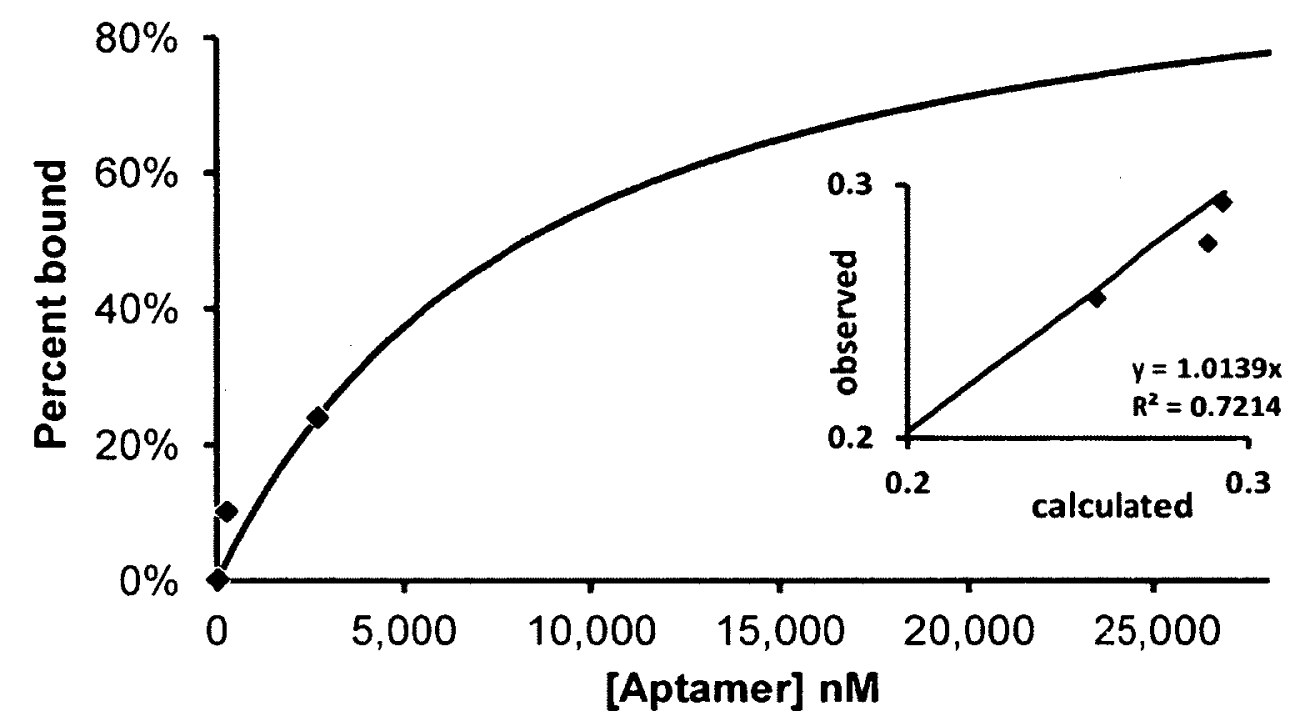

Appx Figure 9. Fluorescent anisotropy binding curve for the DNA form of the RNA dopamine aptamer and tyramine. Anisotropy values were collected using $1 \mu \mathrm{M}$ tyramine which was exposed to increasing concentrations of the aptamer $(0.27,2.7,27$, $270 \mathrm{nM})$. The inset shows the agreement between the observed and the calculated anisotropy values. 